\title{
Indirect Imaging of Cataclysmic Variable Stars
}

\author{
Dissertation zur Erlangung des Doktorgrades \\ der Mathematisch-Naturwissenschaftlichen Fakultäten \\ der Georg-August-Universität zu Göttingen
}

\author{
vorgelegt von \\ Jens Kube \\ aus Kassel
}

Göttingen 2002 

Jens Kube

INDIRECT IMAGING OF

Cataclysmic Variable Stars

Dissertation 



\title{
Indirect Imaging of Cataclysmic Variable Stars
}

\author{
Dissertation zur Erlangung des Doktorgrades \\ der Mathematisch-Naturwissenschaftlichen Fakultäten \\ der Georg-August-Universität zu Göttingen
}

\author{
vorgelegt von \\ Jens Kube \\ aus Kassel
}

Göttingen 2002 
(C) 2002 by Jens Kube. Dissertation, angefertigt an der Universitäts-Sternwarte der Georg-August-Universität zu Göttingen.

D7

Referent: Prof. Dr. Klaus Beuermann

Korreferent: Prof. Dr. Wolfram Kollatschny

Tag der mündlichen Prüfung: 17. Juni 2002 
"Slowly I came to realize that all you have said is true. I learned that you were not keeping knowledge from me deliberately when you taught us so quickly and carefully about the laws and techniques used by the glider-markers on that island. I learned it still more surely when you helped Dondragmer make the differential pulley. I was expecting you to bring up those points in your speech just now; why didn't you? They were good ones.

It was actually when you were teaching us about the gliders that I began to have a slight understanding of what was meant by your term 'science'. I realized, before the end of that episode, that a device so simple you people had long since ceased to use it actually called for an understanding of more of the universe's laws than any of my people realized existed. You said specifically at one point, while apologizing for a lack of exact information, that gilders of that sort had been used by your people more than two hundred years ago. I can guess how much more you know now - guess just enough to let me realize what I can't know.

But you can still do what I want. You have done a little already, in showing us the differential hoist. I do not understand it, and neither does Dondragmer, who spent much more time with it; but we are both sure it is some sort of relative to the levers we have been using all our lives. We want to start at the beginning, knowing fully that we cannot learn all you know in our lifetimes. We do hope to learn enough to understand how you have found these things out. Even I can see it is not just guesswork, or even philosophizing like the learned ones who tell us that Mesklin is a bowl. I am willing at this point to admit you are right; but I would like to know how you found out the same fact for your own world. I am sure you knew before you left its surface and could see it all at once. I want to know why the Bree floats, and why the canoe did the same, for a while. I want to know what crushed the canoe. I want to know why the wind blows down the cleft all the time - no, I didn't understand your explanation. I want to know why we are warmest in winter when we can't see the sun for the longest time. I want to know why a fire glows, and why flame dust kills. I want my children or theirs, if I ever have any, to know what makes this radio work, and your tank, and some day this rocket. I want to know much - more than I can learn, no doubt; but if I can start my people learning for themselves, the way you must have - well, I'd be willing to stop selling at a profit."

The alien creature "Barlennan” in Hal Clement's Mission of Gravity, 1954

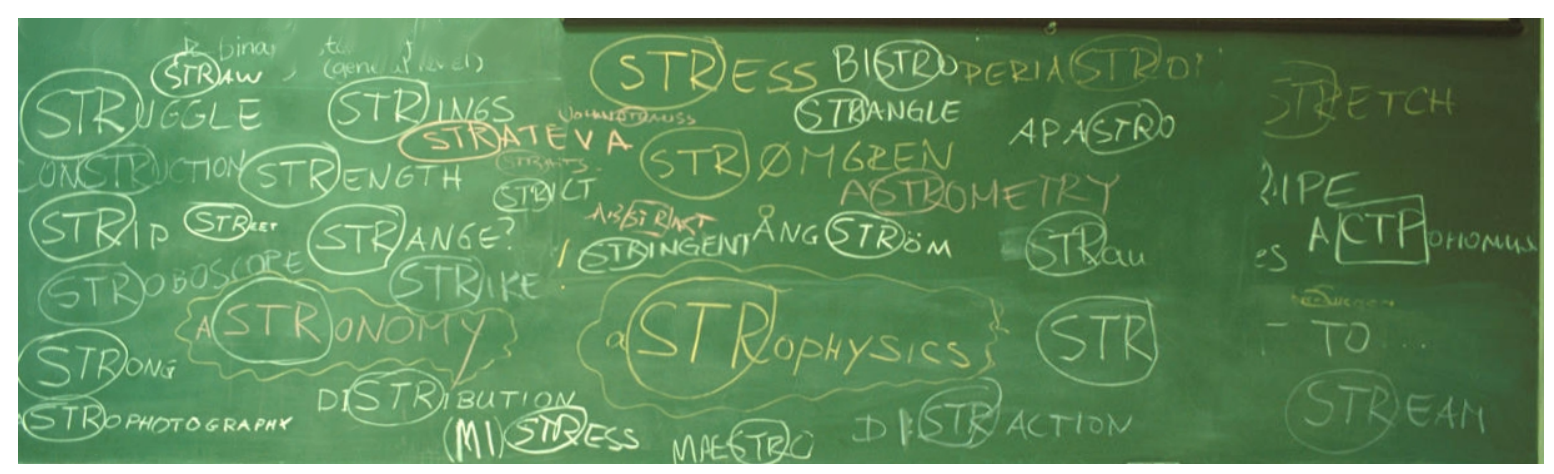

At the Vatican Observatory Summer School 1999 



\section{Contents}

Contents vii

1 Overview 1

2 Introduction I: Cataclysmic Variables 3

2.1 Standard model . . . . . . . . . . . . . . . . . . . 3

2.2 Different types of cataclysmic variables . . . . . . . . . . . . 7

2.3 On the statistics of $\mathrm{CVs} \ldots \ldots \ldots \ldots$

3 Introduction II: Indirect Image Reconstruction $\quad 13$

3.1 What is indirect imaging or tomography? . . . . . . . . . . . . . 13

3.2 1d to $2 \mathrm{~d}$ : classical eclipse mapping . . . . . . . . . . . . . 15

3.3 1d to quasi-3d: "full-orbit eclipse mapping" . . . . . . . . . . . . . . 18

3.4 2d to 2d: classical Doppler tomography . . . . . . . . . . . . . . 18

3.52 d to quasi-3d: orbital mapping . . . . . . . . . . . . . . . . . 23

4 Eclipse Mapping $\quad 25$

4.1 Introduction . . . . . . . . . . . . . . . . . . . . . 25

4.2 The 3 d cataclysmic variable model . . . . . . . . . . . . . . . 27

4.3 Light curve fitting . . . . . . . . . . . . . . . . . . . . . . . . . . . 29

4.4 Test calculations . . . . . . . . . . . . . . . . . . . . . . 32

4.5 Eclipse mapping of UZ For . . . . . . . . . . . . . . . . . . . 37

5 "Full-Orbit Eclipse Mapping" 45

5.1 Method ............................... 45

5.2 Test calculations _. . . . . . . . . . . . . . . . . . 47 
5.3 Full-orbit eclipse mapping of HU Aqr . . . . . . . . . . . . . . . . 52

6 Orbital Mapping $\quad 59$

6.1 Orbital mapping: a combination of eclipse- and Doppler mapping . . . 59

6.2 Test calculations . . . . . . . . . . . . . . . . . . 62

$6.3 \mathrm{HS} 0455+8315 \ldots \ldots \ldots \ldots \ldots \ldots$

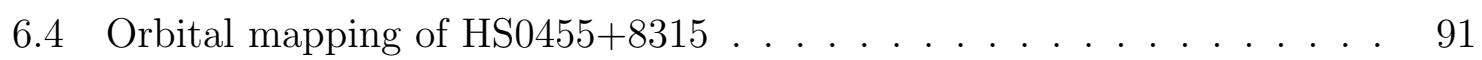

6.5 Orbital mapping of V436 Centauri . . . . . . . . . . . . . . . 101

$\begin{array}{lll}7 & \text { Discussion and Summary } & 109\end{array}$

7.1 The mapping methods . . . . . . . . . . . . . . . . . 109

7.2 Eclipse mapping . . . . . . . . . . . . . . . . . . . . . . . . 109

7.3 Full-orbit eclipse mapping . . . . . . . . . . . . . . . . . . . . . 111

7.4 Orbital mapping . . . . . . . . . . . . . . . . . . . . 112

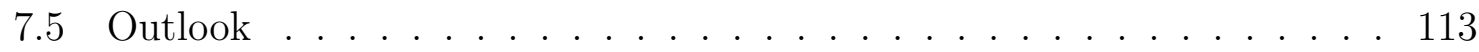

A CVcat - An Interactive Database of Cataclysmic Variables and Re$\begin{array}{ll}\text { lated Objects } & 115\end{array}$

A.1 The concept . . . . . . . . . . . . . . . . . . . . . . . 115

A.2 Technical realization . . . . . . . . . . . . . . . 116

A.3 Prospects . . . . . . . . . . . . . . . . . 116

$\begin{array}{lr}\text { B Symbols and Acronyms } & 119\end{array}$

$\begin{array}{lll}\text { Bibliography } & \text { I }\end{array}$

List of Tables $\quad$ VII

$\begin{array}{ll}\text { List of Figures } & \text { IX }\end{array}$

$\begin{array}{ll}\text { Publications } & \text { XIII }\end{array}$

$\begin{array}{ll}\text { Acknowledgements } & \text { XV }\end{array}$

$\begin{array}{ll}\text { Curriculum Vitae } & \text { XVII }\end{array}$ 


\section{Chapter 1}

\section{Overview}

Interacting binary stars are of great importance for the analysis of various astrophysical problems. First, they offer conditions which are very difficult to produce in a terrestrial or artificial laboratory environment. Mass transfer rates with up to several $10^{-8}$ solar masses per year - that is $\approx 10^{11}$ metric tons of matter per second! - from one star to another, magnetic fields of up to $230 \mathrm{MG}$ in the "polars", nova eruptions and other drastic features characterize a field of physics, which allows to study the behaviour of matter well over the limits accessible on earth or in normal stars. Second, accretion that is the general term for addition of mass - is quite common in other astrophysical surroundings like in the core of active galaxies, where supermassive black holes act as the sink of material or in the process of star formation and the creation of planets. Third, the cataclysmic variables, the subclass of interacting binaries this thesis focuses on, have orbital periods of a few hours which allow to analyze their spectra from different viewing angles during a short observing time, allowing for tomographical image reconstruction.

In this thesis I use the third of the above viewpoints. Reconstruction of spatially resolved images of cataclysmic variable stars $(\mathrm{CVs})$, from which time dependent intensities or spectra are available, has a nearly 20 year long tradition. In several papers, Keith Horne and Tom Marsh founded the methods called eclipse mapping (Horne, 1985) and Doppler mapping (Marsh and Horne, 1988). In their original works, these methods were applied to accretion disks in CVs. Since then these basic techniques have led to more developed methods: Physical parameter eclipse mapping of accretion disks (Vrielmann et al., 1999), in which not only the intensities but a complete set of physical parameters is mapped on the disk, skew mapping of secondary stars in CVs (Smith et al., 1998), Doppler mapping of star spots (Vogt and Penrod, 1983), which is an even older application of Doppler mapping than the paper by Marsh and Horne quoted earlier, and the most recent idea of Kepler tomography (Hoffmann et al., 2002), where the mapping is done in the frame of a mass element rotating with its Kepler velocity around the white dwarf. Also non-disklike accretion phenomena have been mapped: 
The accretion streams in highly magnetic CVs, where due to the strong magnetic field of the white dwarf the creation of an accretion disk is prevented, have been under investigation since seven years, starting with a publication by Pasi Hakala (1995). CVs are described in some detail in Chapt. 2, an overview over the core techniques of image reconstruction is given in Chapt. 3 .

I developed, analyzed, and applied three different new mapping methods. The first two of these basically do a forward synthesis of light curves of magnetic systems. The third method uses spectra of either accretion disks or magnetic systems. These synthetic data are modified until the light curves or spectra fit to the observed data. The fitting algorithm uses an evolution strategy for the optimization process.

In Chapt. 4 I describe my approach to eclipse mapping of the accretion stream in magnetic CVs. This method is applied to UV data of UZ For taken with the Hubble Space Telescope, HST. In Chapt. 5, I extend the eclipse mapping method to use data taken over a full binary star orbit. This approach is applied to optical data of another magnetic CV system, HU Aqr.

In Chapt. 6, I add a spectral resolution as a second dimension to the dataset. I apply the spectral fitting method, which I dubbed "orbital mapping", to two non-magnetic CVs: With data of the newly discovered HS0455+83, an SW Sex system, I try to substantiate the stream overflow model of this subtype of CVs. The SU UMa System V436 Cen is analyzed for the radial intensity gradient in the accretion disk and absorption signatures which cannot be explained by a simple disk model.

Finally, Chapt. 7 contains a discussion of the new mapping methods in comparison to the established ones. A summary of this thesis is given as well as an outlook to the future of indirect imaging in CVs.

I complete this thesis with the description of a data base of cataclysmic variables, which I developed as a service to the CV community (App. A). 


\section{Chapter 2}

\section{Introduction I: Cataclysmic Variables}

In this chapter I give a brief summary on the nature of the objects which are analyzed in this thesis, cataclysmic variables $(\mathrm{CVs})$; this summary consists of the description of the standard model (Sect. 2.1) and of the different subtypes of CVs (Sect. 2.2). Using the newly developed CV data base (CVcat, App. A), I present three examples of statistical work on CVs (Sect. 2.3).

Recently, some very good reviews on cataclysmic variables $(\mathrm{CVs})$ were published: A good introductory work on CVs is Coel Hellier's book (2001), while the classic book from Brian Warner (1995a) still is the reference when it comes to the details. Jósef Smak's (2001) contribution to the EADN School XII proceedings (Lázaro and Arévalo, 2001) also gives a good introduction to the CV standard model, whereas Tom Marsh (2001) in the same proceedings focuses on the observations of CVs. The most recent results of $\mathrm{CV}$ research will be found in the upcoming proceedings of the $\mathrm{CV}$ conference which was held in Göttingen, August 5-10, 2001 (Gänsicke et al., 2002a).

\section{$2.1 \quad$ Standard model}

Cataclysmic variables are binary stars, which consist of a white dwarf (WD, primary) and a late main sequence donor star (red dwarf, secondary) which fills its Roche lobe. Matter from the secondary flows over the gravitational wall and falls towards the WD. In most CVs, the mass transfer rate is $\dot{M} \approx 10^{-11} \ldots 10^{-8} M_{\odot} \mathrm{yr}^{-1}$ (Smak, 2001). The transfer of matter leads to many interesting - sometimes catastrophic, hence the term "cataclysmic" - effects, which happen on timescales of seconds (accretion of blobs of material) up to centuries (nova eruptions). 
Common characteristics (apart from the WD/red dwarf nature of the components) of CVs are:

- Short orbital periods from $59 \mathrm{~min}$ for V485 Cen (Augusteijn et al., 1993) to typically $10 \mathrm{~h}$, with the longest known period of $5.7 \mathrm{~d}$ for V1017 Sgr (Sekiguchi, 1992). CVs with helium donor stars (AM CVn systems) have even shorter orbital periods down to $10 \mathrm{~min}$ or below.

- WD masses from $0.16 M_{\odot}$ (Shafter and Szkody, 1984) in T Leo to the theoretical maximum of $1.4 M_{\odot}$ in V592 Cas (Huber et al., 1998) with most $M_{1}$ in the range of $0.4 \ldots 1.3 M_{\odot}$. The measurement of WD masses is a delicate task, one should use these values with care.

- Secondary masses in the range from $0.03 \pm 0.02 M_{\odot}$ in WZ Sge (Ciardi et al., 1998) to $1.1 M_{\odot}$ in SY Cnc (Shafter, 1983). On the statistics of $M_{2}$, see also Sect. 2.3.2).

- accretion disks (except for the magnetic systems, see Sect. 4.1).

\subsubsection{The Roche lobe}

The Roche potential is calculated by considering the gravitational forces of the two stellar components and the centrifugal force which acts on a co-rotating test particle. With $a$ being the distance between the centers of mass, $M_{1,2}$ the components' masses, $r_{1,2}$ the distances from the centers of the components, $G$ gravitational constant (see App. B), one finds:

$$
\Phi(x, y, z)=-G\left(\frac{M_{1}}{r_{1}}+\frac{M_{2}}{r_{2}}+\frac{M_{1}+M_{2}}{2 a^{3}}\left[\left(x-\frac{a M_{2}}{M_{1}+M_{2}}\right)^{2}+y^{2}\right]\right)
$$

Define

$$
\begin{aligned}
Q & :=\frac{M_{1}}{M_{2}} \\
M & :=M_{1}+M_{2} \\
r_{1 / 2}^{\prime} & :=\frac{r_{1 / 2}}{a} \\
\left(x^{\prime}, y^{\prime}, z^{\prime}\right) & :=\frac{(x, y, z)}{a}
\end{aligned}
$$

Factoring out $M_{1} / a$ in Eq. (2.1) leads to:

$$
\begin{aligned}
\Phi(x, y, z) & =-\frac{G M_{1}}{a}\left[\frac{1}{r_{1}^{\prime}}+\frac{1}{Q r_{2}^{\prime}}-\frac{x^{\prime}}{Q}+\frac{1}{2}\left(x^{\prime 2}+y^{\prime 2}\right)(1+1 / Q)\right] \\
& =-\frac{G M_{1}}{a} \cdot f\left(\frac{x}{a}, \frac{y}{a}, \frac{z}{a}, Q\right)
\end{aligned}
$$




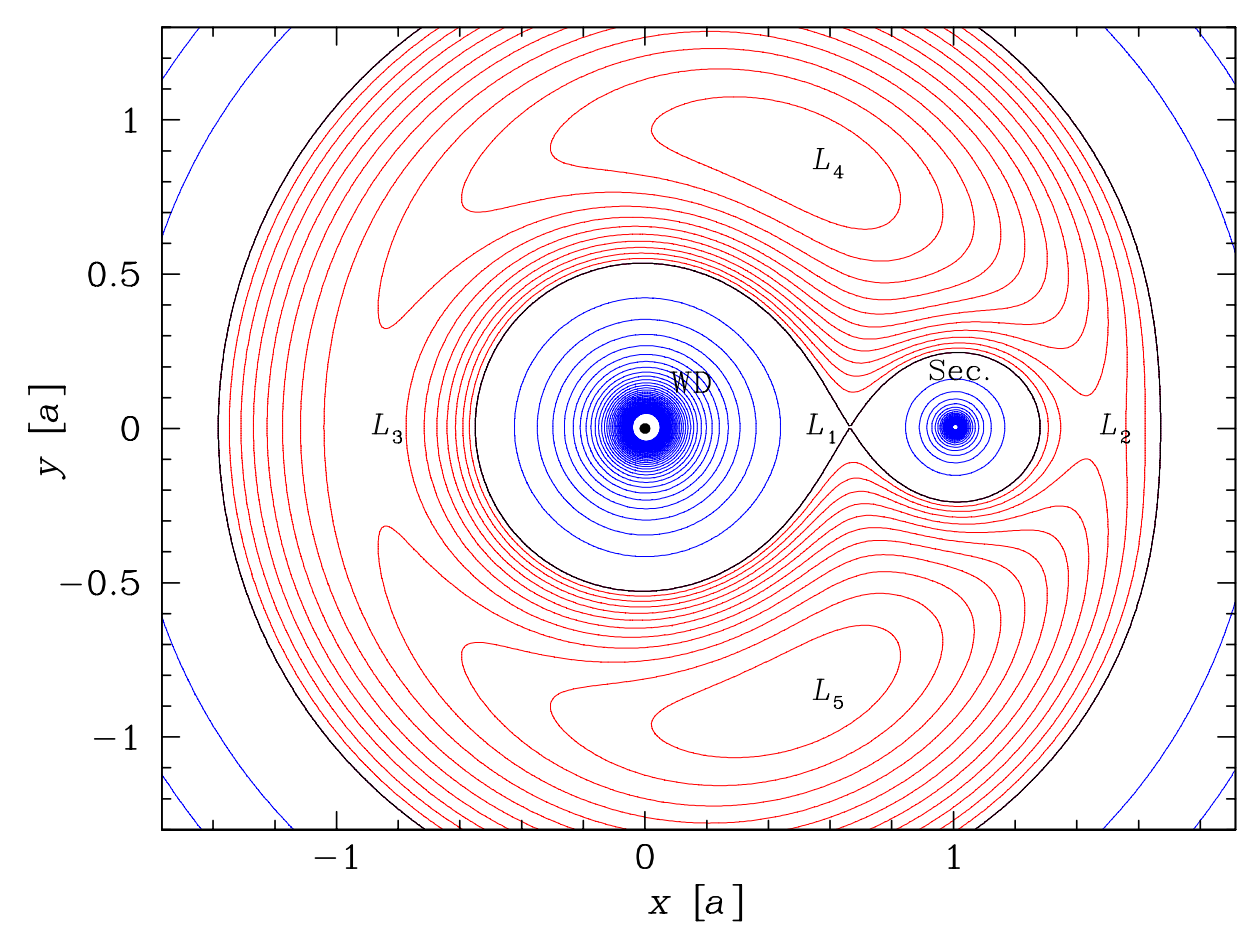

Figure 2.1: Roche equipotential surfaces for a mass ratio of $Q=M_{1} / M_{2}=5$. Shown are some equipotential lines in the orbital plane. The Roche lobe is drawn with a thicker line. The secondary star (Sec.) in a CV fills its Roche lobe, while the small white dwarf (WD) sits inside a larger Roche lobe. Red lines indicate a potential above the $L_{1}$-equipotential surface, blue lines a potential below that. For clarity, the potential spacing between two blue lines is ten times as big as between two of the red lines.

From the last equation, it is easy to see that the shape of the Roche potential is a function of the mass ratio only. The orbital distance, $a$, is a scaling factor, as is the mass of the components. Close to the WD, one can approximate the potential as the central potential of a single point mass, allowing an easy formulation for the Keplerian velocity of a particle orbiting around the WD as shown in Eq. (2.9).

The Roche lobe is the equipotential surface of the Roche potential which crosses the $L_{1}$-point, the so-called "inner Lagrangian point". At this point, the local maximum of the potential between the two stars (Fig. 2.1) is located. This is the maximum size a star can have without losing mass to its companion. There are systems where none of the stars fills its Roche lobe ("detached system"), systems where one star fills its Roche lobe ("semi-contact system", that is the field of CVs), systems where both stars fill their Roche lobes ("contact systems") or even those where the stars have a common envelope which extends over the Roche lobe ("over-contact systems"). The over-contact systems can extend to the lobe passing the $L_{2}$ point at maximum, larger systems would lose mass to the interstellar medium. 


\subsubsection{Mass transfer}

What is neccessary to drive mass transfer? Mass transfer happens if the size of the secondary star equal to or slightly larger than its Roche lobe. Matter will fall from the $L_{1}$-point towards the white dwarf. Since most of the matter hits the white dwarf and is kept there, this process is called "accretion". Due to the conservation of angular momentum, the matter does not fall directly towards the white dwarf. In non-magnetic systems, accretion disks forms. Viscosity effects transfer angular momentum to larger disk radii, while the matter is transported towards the white dwarf (Shakura and Sunyaev, 1973).

A mass ratio $Q<1$ would result in a catastrophic mass transfer burst: Matter from the heavier donor star would - after being accreted - be further away from the center of gravity than before. Hence it would gain angular momentum. To conserve the total angular momentum, the system would have to shrink, bringing both stars closer together, and by reducing the size of the Roche lobe of the secondary allowing even more matter to overflow.

In $\mathrm{CVs}$, the mass ratio $Q=M_{1} / M_{2}$ is always larger than unity. Mass transfer from the lighter donor star to the white dwarf causes a angular momentum loss of the accreted matter, which has to be compensated by moving the stars further away. To keep matter flowing, the size of the orbit has to shrink, in other words, angular momentum has to be extracted from the system. There are two main mechanisms which are believed to be responsible for the angular momentum loss: magnetic braking and gravitational radiation.

Magnetic braking is the more efficient mechanism at long orbital periods. It governs the development of a $\mathrm{CV}$ from its birth at a orbital period of $\approx 10 \mathrm{~h}$ down to the upper end of the period gap at $P \approx 3 \mathrm{~h}$. The basic idea is that a stellar wind emanates from the Alfén surface of the secondary thereby exerting a braking torque on the star which extracts angular momentum from the secondary. Because of the tidal interaction with the primary, the rotation of the secondary is kept synchronous with the orbital motion. So the system has to reduce its orbit to adjust to the angular momentum drain. That leads to a shorter period. The mass transfer rate imposed by the magnetic braking is

$$
\dot{M}_{\mathrm{mag}} \approx 1.7 \ldots 3.7 \times 10^{-9} M_{\odot \mathrm{yr}^{-1}}
$$

for a $P=4$ h system (Rappaport et al., 1983; McDermott and Taam, 1989). For shorter orbital periods, the magnetic braking becomes very inefficient (Spruit and Ritter, 1983). Since the mechanism for magnetic braking depends on the stellar wind and the stellar magnetic field, it's difficult to calculate the mass-loss rate exactly.

Gravitational braking extracts energy from the binary system by gravitational radiation. The accretion rate for a $P_{\text {orb }}=2 \mathrm{~h}$ system is

$$
\dot{M}_{\text {grav }} \approx 5 \times 10^{-11} M_{\odot} \mathrm{yr}^{-1}
$$


Gravitational radiation is responsible for the braking of CVs with orbital periods below $3 \mathrm{~h}$.

The CV period distribution shows a depletion in the range $P_{\text {orb }}=2 \ldots 3 \mathrm{~h}$ ("period gap"). The interpretation is basically that systems evolving down to periods of $3 \mathrm{~h}$ switch off their mass transfer since magnetic braking becomes too inefficient to keep accretion running. Additionally, accretion has driven the secondaries out of their thermal equilibrium, which is now re-adjusted. This leads to a shrinking of the secondaries' radii well below the Roche lobe size, shutting of accretion completely. These systems evolve slowly down to $2 \mathrm{~h}$ due to gravitational braking. At $P_{\text {orb }} \approx 2 \mathrm{~h}$ the Roche lobe has shrunk enough to switch the accetion on again, now with the low accretion rate imposed by gravitational braking.

\section{Excursion: Some useful formulae}

When working with positions, velocities etc. in a CV system, one may find the following collection of formulae (given here without a proof) handy. $M=$ $M_{1}+M_{2}, Q=M_{1} / M_{2}, P=P_{\text {orb }}$; for a complete list of the symbols, see App. B.

$$
\begin{aligned}
a(M, P) & =\left(\frac{G M P^{2}}{4 \pi^{2}}\right)^{\frac{1}{3}} & & \text { orbital separation } \\
a_{1} & =\frac{a}{1+Q} & & \text { distance from WD to c/g } \\
v_{\text {kep }}(r) & =\sqrt{\frac{G M}{r}} & & \text { Keplerian velocity } \\
v_{\text {disk,kep }}(r) & \approx \sqrt{\frac{G M_{1}}{r}} & & \text { Keplerian velocity close to WD }
\end{aligned}
$$

\subsection{Different types of cataclysmic variables}

The Cataclysmic Variables are not a homogenous group. A possible grouping of CVs is "dwarf novae", "novalikes", "magnetic systems" and "novae", which all have subgroups. I will not discuss the AM CVn stars (helium secondary, hence shorter periods) and the supersoft X-ray binaries (SSXBs, steady hydrogen burning on the surface of the primary star) here.

As discussed before, the accretion rates differ between different individual CVs. Many effects that allow to distinguish between dwarf novae (DN) and novalike variables (novalikes, NL) are a direct result from different accretion rates. The dwarf novae are systems with low accretion rates compared to the novalikes. 
Dwarf novae experience quasi-regular outbursts of $1 \ldots 8$ magnitudes. The typical interval between two outbursts is $4 \ldots 450$ days. The different subtypes of dwarf novae are named after their reference objects:

SS Cyg Sometime these stars are also referred to as U Gem stars, naming another famous member of this most regular group of CVs. The outbursts come pretty regularly, showing only three different shapes (short outburst, long outburst, slow rising outburst). SS Cyg systems are found above the period gap.

Z Cam stars are at the edge between novalikes and dwarf novae, switching irregularly from a standstill in magnitude to the outburst behaviour seen in SS Cyg systems and back. These systems are also found above the period gap, but the accretion rate is higher than in the SS Cyg systems.

SU UMa In these stars we observe a "superoutburst" after some SS Cyg-like outbursts, which is slightly brighter than the normal outbursts and lasts longer. During that outburst, "superhumps" in the light curve are seen which have a period some percent longer than the orbital period. The interpretation is that some outbursts cause the disk to become elliptical, with the precession seen as the superhump. The time between two superoutbursts is called "supercycle" and lasts typically $200 \ldots 1000$ days. SU UMa systems have orbital periods below the period gap with four exceptions: three systems are found in the gap (NY Ser, TU Men, V405 Vul), and one well above the gap $\left(P_{\text {orb }}=254.304 \mathrm{~min}\right.$, ES Dra).

WZ Sge These are SU UMa Systems with supercycles of well over 1000 days (lowest accretion rate), normal outbursts are rare. In WZ Sge itself no normal outburst has been observed.

ER UMa With supercycle lengths of less than 100 days, this groups forms the lower end of the superoutbursters (highest accretion rate in the group of the superoutbursters).

Novalikes show irregular variations. Older classifications wrongly included the group of magnetic objects into the novalikes, which are now considered a separate class. The novalikes fall into two groups, again named after their prototypes:

VY Scl These are sometimes referred to as "anti-dwarf novae". Their normal state of accretion is the high state with a hot disk. Sometimes their accretion rate drops. Then, they are found in a low state with low brightness due to the low accretion rate. Differently from Z Cam systems, VY Scl stars do not show outbursts while they are in the low state. RX And, however, has been identified as a transitional system between Z Cam and VY Scl: It shows outburst behaviours of both classes (Schreiber et al., 2002), which are attributed to accretion rate changes. 
UX UMa systems do not show low states.

In the class of novalikes, about a third of the systems shows a behaviour which is known as the SW Sex phenomenon. The properties, which classify a star as an SW Sex systems are (Hoard, 1998): Strong He II $\lambda 4686$ emission, assymetric disk emission and variable line strength over the orbit. SW Sex systems show a zero velocity absorption in the core of the Balmer and He I emission lines around orbital phase $\phi=0.5$.

The magnetic systems are categorized by their highly magnetic white dwarf. The subgroups of the magnetic systems are distinguished by the synchronism or nonsynchronism of the white dwarf with the orbital motion:

AM Her systems or "polars" have a white dwarf with a very high magnetic field strength $(7 \ldots 230 \mathrm{MG})$. The rotation period of the white dwarf is locked to the orbital motion. There are some AM Her systems which are not totally synchronized. Polars have no accretion disks. A more detailed description of AM Hers is given in Sect. 4.1.

DQ Her stars do not rotate synchronously. The rotation period of the white dwarf is much shorter than the orbital period, typically $P_{\text {spin }} / P_{\text {orb }} \approx 1 / 10$. The WD spins significantly faster in AE Aqr, where $P_{\text {spin }} \approx 1 / 1075 \cdot P_{\text {orb }}$ (de Jager et al., 1994), while the spin in EX Hya is very slow, $P_{\text {spin }} \approx 1 / 1.47 \cdot P_{\text {orb }}$ (Bond et al., 1988).

Novae are categorized by at least one very strong eruption of $6 \ldots 19$ magnitudes. The nova eruption is caused by nuclear fusion of the accreted hydrogen on the surface of the white dwarf. Most novae are observed only once, but the hydrogen burning model would require them to have recurrent eruptions, since after one hydrogen explosion the surface of the WD can again accumulate matter until the next eruption happens. The timescale between two eruptions may just be too long to allow for a second observed eruption yet. In fact, there are some recurrent novae. Many belong to systems with long orbital periods $\left(P_{\text {orb }}>1000 \mathrm{~min}\right)$ or to non-CVs with a giant donor star. However, $\mathrm{T}$ Pyx is a CV for which 5 nova eruptions are recorded (we are expecting eruption No 6 which is overdue).

An overview of the different types of $\mathrm{CVs}$ and their relative numbers is given in Fig. 2.2 .

\subsection{On the statistics of CVs}

Now that I have described the objects in general, I will present some statistics, which are based on the data contained in CVcat (see App. A, CVcat was formerly dubbed "TPP"), a data base project which was developed with the aim to contain all published data on CVs (Kube et al., 2002). The following statistics were extracted from CVcat 


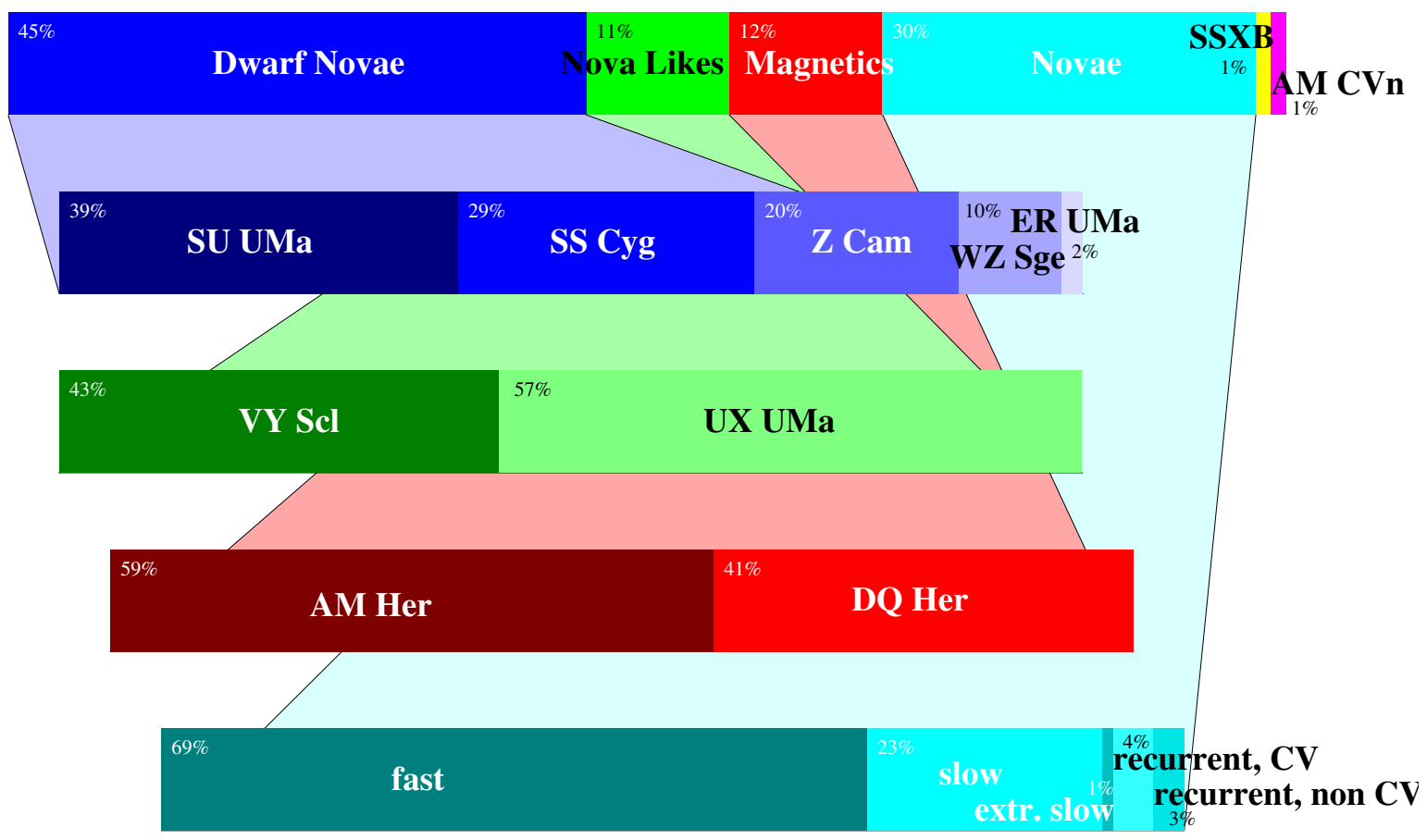

Figure 2.2: Relative numbers of CVs of the different types and hierarchical grouping of the subtypes. Taken from the CVcat data base in January 2002.

on January 14, 2002. Since CVcat is an interactive catalogue where all registered users may change entries, the following statistics will differ from later mining in the data base.

\subsubsection{Number of objects}

The CVcat-catalog holds data for 967 objects classified as CVs. From these, 421 are dwarf novae, 103 are novalikes, 115 are magnetic systems (either polars or intermediate polars), 280 are novae, 12 are SSXBs, and 8 are AM CVn stars. From the non-magnetic systems, roughly 20 to $40 \%$ of the systems are classified in more detail. Assuming, that no preference in the classification of specific subclasses has been made, the statistics of the systems looks as shown in Fig. 2.2. Note that all magnetic systems indeed have a subclass categorization either as a polar or an intermediate polar.

In the NL class, the subclassification is least developed. There are 67 systems which are tagged as novalikes without any further category. From the 13 UX UMa-system, none is marked as a star showing SW Sex features, while half of the VY Scl systems are also SW Sex systems. From the uncategorized novalikes, 8 (12\%) are marked as SW Sex, so the statistics of which systems can be SW Sex stars is far from being complete. 


\subsubsection{Secondary star mass}

In Fig. 2.3 I have plotted the derived secondary star masses over the orbital period, as known to the CVcat data base. A linear trend with

$$
\frac{M_{2}}{M_{\odot}} \approx 0.11 P / \mathrm{h}-0.06
$$

is clearly visible. This is predicted by theoretical considerations, where the reasoning is roughly this (Frank et al., 1992): Using an approximation for the Roche geometry, valid for $1.3 \lesssim q \lesssim 10$, and Eq. (2.6), one finds that the mean density of a Roche lobe filling star is a function only of the orbital period. With the knowledge of the lower main-sequence $M / R$ relation (Kippenhahn and Weigert, 1990; Baraffe et al., 1998), one then finds

$$
\frac{M_{2}}{M_{\odot}} \approx 0.11 P / \mathrm{h}
$$

A more detailed analysis of secondary star masses showed slightly different periodmass-relations (Smith and Dhillon, 1998):

$$
\begin{aligned}
& \frac{M_{2}}{M_{\odot}}=(0.038 \pm 0.003)(P / h)^{(1.58 \pm 0.09)} \\
& \frac{M_{2}}{M_{\odot}}=(0.126 \pm 0.011) P / h-(0.11 \pm 0.04)
\end{aligned}
$$

\subsubsection{Period distribution}

Another feature is very remarkable in Fig. 2.3: The objects are not distributed uniformly over the different orbital periods. Obviously, there are a pretty sharp lower and a not so sharp upper limits of the orbital periods. Moreover, in the range of $P \approx 2 \mathrm{~h} \ldots 3 \mathrm{~h}$, there are very few systems. This range is referred to as the "period gap". Since the processes that lead a CV to change its period due to the braking processes described above work on long timescales compared to the observation baseline we have (roughly one hundred years now), the period distribution is used to determine the evolution timescales and test evolution models for the CV population. Without going into details, I may just state that the evolutionary scenarios so far do not agree on all aspects on the period distribution, especially on the low-period cutoff (Kolb, 2002; King and Schenker, 2002; Schenker and King, 2002). 


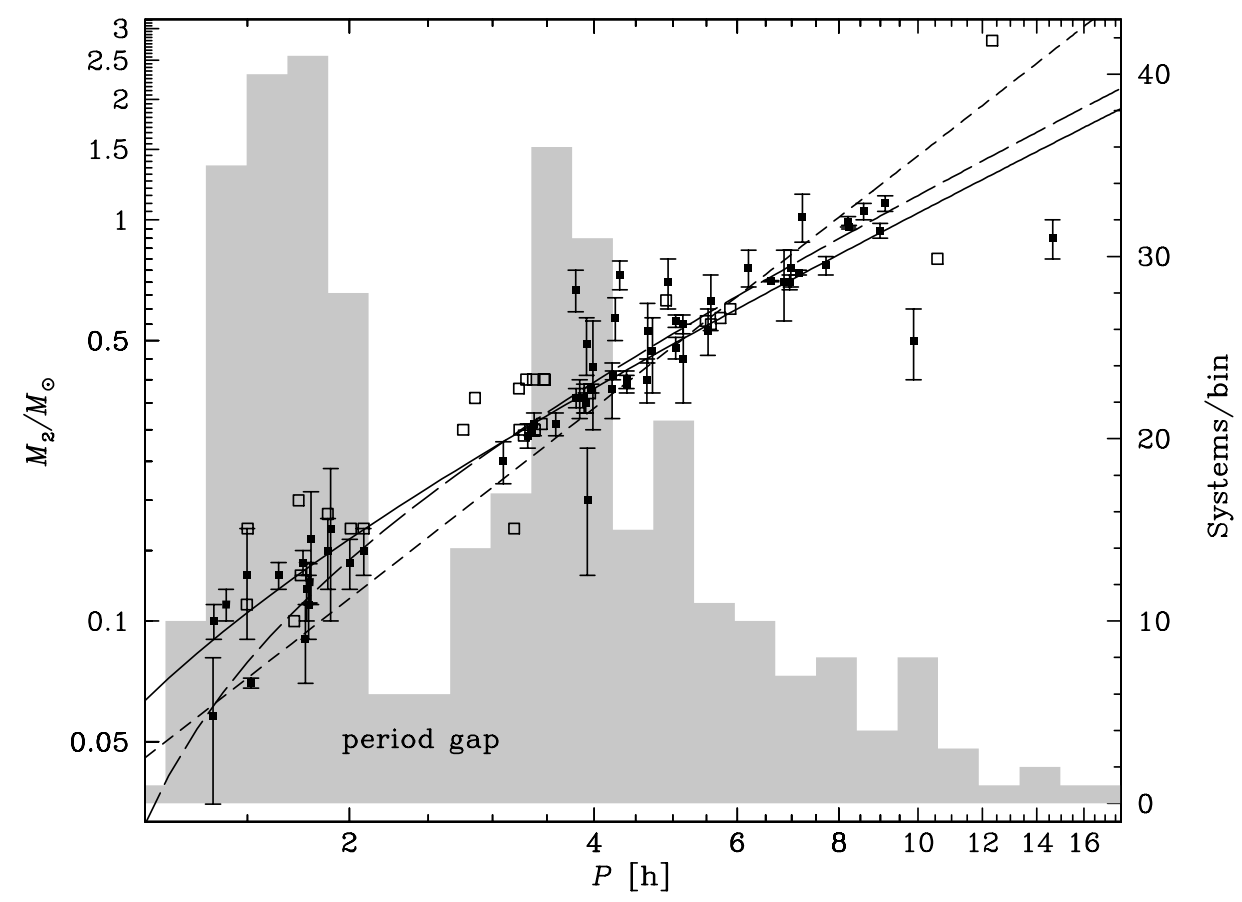

Figure 2.3: Secondary mass plotted over orbital period (points) and period histogram (gray bars). All CVs with known orbital period and secondary mass, taken from the CVcat data base. The full line shows the ratio from Eq. (2.10), the short dashed line visualizes Eq. (2.12), the long dashed line Eq. (2.13). Full boxes and error bars are used for systems with estimated errors in $M_{2}$, open boxes are drawn for secondary star masses with an unknown error range. The sample of $M_{2} / P$ contains about $10 \%$ of all known CVs, the histogram nearly $50 \%$. 


\section{Chapter 3}

\section{Introduction II: Indirect Image Reconstruction}

In this chapter I give the foundations and motivations for my work. A very brief overview of the well-known methods of astrotomography is given as well as an introduction of the new methods developed in this work. A more detailed description of eclipse mapping, full-orbit eclipse mapping, and orbital mapping can be found in the subsequent chapters.

\subsection{What is indirect imaging or tomography?}

Astronomical objects are large and bright (except for gasous nebulae, which are still large, but very dim). Unfortunately, they are very far away, which makes their apparent sizes and brightnesses rather small. The CVs on which this work focuses are typically as bright the sun when they are in their high state, and their orbital separation $a$ is of the order of the solar radius. Since they are located in our vicinity inside the Milky Way, a typical distance is of the order of $d \approx 100 \mathrm{pc}$. That leads to a very small angular size $\alpha$ :

$$
\alpha=\arctan \frac{a}{d} \approx \text { some } \mu \operatorname{arcsec}
$$

There is no optical telescope with such an angular resolution, even the new VLTI goes down to 1 marcsec at its best (Glindemann et al., 2000). To resolve spatial features inside a CV, other methods are necessary. Since one uses non-spatial, i.e. time-resolved or spectroscopically-resolved (or both), information to derive information about the spatial features of a remote astronomical system, the most general term covering all these methods is "indirect imaging". Sometimes all indirect imaging techniques are referred to as tomography, but the term "tomography" is used mostly for the Doppler tomography method. All methods which use $n$ images of $d^{\prime}$-dimensional cross-sections 


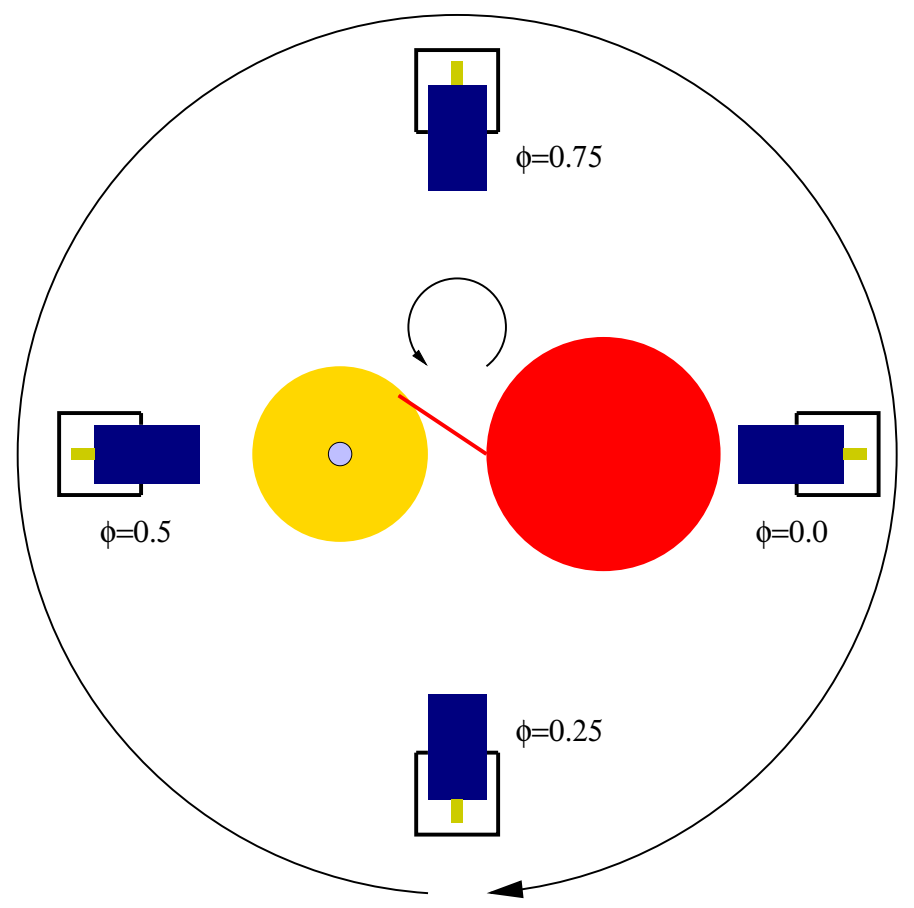

Figure 3.1: The principle of tomography: Observe (with the blue telescope) from different positions (outer circle) - rotate the object (inner circle).

of an $d$-dimensional object to reconstruct an $d^{\prime}+m$-dimensional image of that object deserve to be called "tomographic". $m$ is either 1 or 2 . For $m=1$, one can understand tomography as a transformation of the time dimension which allows the $n$ different observing angles into a spatial dimension. For $m=2$ some restrictions of where emission can come from have to be made. In the following, 1-dimensional data stands for time resolved $d^{\prime}=0$-data (light curves), 2-dimensional data for time resolved $d^{\prime}=1$-data (trailed spectra).

The most recent and most complete review of all astro-tomography methods is given in the proceedings of the astrotomography workshop in Brussels, held in July 2000 (Boffin et al., 2001). I will sum up the basic ideas behind the progenitor methods to my newly developed "full-orbit eclipse mapping" and "orbital mapping" methods.

In Fig. 3.1 the main principle of tomographical methods is sketched, which use the orbital rotation of the observed object as the generator of the $n$ different images ${ }^{1}$. At phase $\phi=0$, the donor star (red) is in between the observer and the white dwarf (light blue) and the accretion structure (yellow). Hence, the observer just sees the backside of the red star. A quarter of an orbit later, the observer looks at the system laterally, while at $\phi=0.5$, the primary star and the accretion structure are in front, maybe eclipsing some parts of the donor star. At $\phi=0.75$, the situation is similar to $\phi=0.25$.

\footnotetext{
${ }^{1}$ This excludes the class of "echo mapping" methods (Horne et al., 1991; Horne, 2001; O'Brien and Horne, 2001), where runtime differences from reprocessed radiation are used for scanning the object.
} 
Remember that it is not possible to spatially resolve the system, hence the observer sees the sum of all emissions from the system visible from his position.

It is easy to understand that the data set consisting of $n$ images with $d^{\prime}$ dimensions ( $d^{\prime}=0$ for light curves, $d^{\prime}=1$ for spectra) allows to reconstruct the location or the emission characteristics of the system, no matter how far it is away. The distance does not limit the resolution of indirect images except by the signal to noise ratio.

\section{$3.21 \mathrm{~d}$ to $2 \mathrm{~d}$ : classical eclipse mapping}

Parts of this section are published in my contributions to the Brussels conference (Kube, 2001).

Eclipse mapping is the tomographical method which is easiest to understand. Typically, one uses the brightness of the system in a specific wavelength range observed at different times which are transformed into orbital phases. Using the nomenclature from above, the dimensionality of the single images is $d^{\prime}=0$, and the time resolution is described by $n$ images. The indirect image can have $d=1$ dimension, if no other assumptions are made. The additional assumption involves the secondary star as an optically thick geometric obstacle for the radiation. The eclipse mapping method is based on the fact that, in an orbiting system with high inclination, an object like the accretion stream is gradually eclipsed by another object, e.g. the secondary star. This allows to constrain the location of the observed emission to a geometrical figure which is the overlap of the physical model geometry with the "shadow" of the obstacle, i.e. the region that remains hidden behind the secondary star

At a given time - written as orbital phase $\phi$ - one observes the flux $F(\phi)$. If $\mathcal{V}(\phi)$ are all visible parts of emitting objects of the binary system, we can write

$$
F(\phi)=\int_{\mathcal{V}(\phi)} I(\vec{r}) f(\vec{n}(\vec{r})) \mathrm{d} S
$$

where $I(\vec{r})$ is the intensity of a surface element at the position $\vec{r}$ and $\vec{n}(\vec{r})$ is the normal vector of this surface element. $f(\vec{n}(\vec{r}))$ is the function describing the geometrical emission characteristics of the surface. For optically thick emission with no limb darkening, $f(\vec{n}(\vec{r}))$ becomes $\cos (\alpha)$, where $\alpha$ is the angle between $\vec{n}(\vec{r})$ and the observer.

Since all mapping codes use grids of $N$ finite surface elements, we can write the continuous formulation in Eq. (3.2) discretely as

$$
F(\phi)=\sum_{i \in \mathcal{V}(\phi)} I_{i} A_{i}(\phi)
$$

with $I_{i}$ being the intensity of the $i$-th surface element $(0<i<N), A_{i}(\phi)$ the effective surface of this surface element at orbital phase $\phi$ and $\mathcal{V}(\phi)$ the set of visible surface elements at orbital phase $\phi$. 
The physical model about the nature of the mapped object is used to calculate the matrix $A_{i}(\phi)$. Such a model has to give the information about the visible surface elements $\mathcal{V}(\phi)$ and about their apparent sizes. Strictly speaking, arbitrarily complicated angular dependencies of the emission can be included in $A_{i}(\phi)$.

In the case of my work, the computer code CVMOD calculates $A_{i}(\phi)$ and makes a forward synthesis of $F_{\text {model }}(\phi)$ which is compared to the observed $F(\phi)$. By changing the intensity vector $I_{i}$, the difference between $F_{\text {model }}(\phi)$ and $F(\phi)$ is minimized. The intensity vector $I_{i}$ which belongs to the best fitting $F_{\text {model }}(\phi)$ is the map which is the aim of the mapping process. Since the number of data points typically is somewhat smaller than the number of surface elements, the map has to be smoothed, which is done by the means of an entropy maximization. The process is described in more detail in Sect. 4.3 (pp. 29 ff.).

\subsubsection{Artifacts and the size of the surface elements}

For the following discussion, I consider the case of the diskless magnetic CVs (polars, see Sect. 4.1 on p. 25). It also applies - with the adequate adaption - to the case of eclipse mapping in disk systems.

Let $\Delta F_{1,2}$ be the flux difference between two different orbital phases $\phi_{1}$ and $\phi_{2}$. Using Eq. (3.3), $\Delta F_{1,2}$ reads like

$$
\Delta F_{1,2}:=F\left(\phi_{1}\right)-F\left(\phi_{2}\right)=\sum_{i \in \mathcal{V}\left(\phi_{1}\right)} I_{i} A_{i}\left(\phi_{1}\right)-\sum_{i \in \mathcal{V}\left(\phi_{2}\right)} I_{i} A_{i}\left(\phi_{2}\right)
$$

For small phase differences, say $360^{\circ} \cdot\left|\phi_{1}-\phi_{2}\right|<10^{\circ}$, the projected surface areas don't differ much: $A_{i}\left(\phi_{1}\right) \approx A_{i}\left(\phi_{2}\right)=: A_{i}$. Let's assume for the following that $\phi_{1}$ and $\phi_{2}$ are close to 0.95 , which means that we are looking at the eclipse ingress of the accretion stream. With $\mathcal{I}_{1,2}:=\mathcal{V}\left(\phi_{1}\right) \backslash \mathcal{V}\left(\phi_{2}\right)$, Eq. (3.4) becomes

$$
\Delta F_{1,2}=\sum_{i \in \mathcal{I}_{1,2}} I_{i} A_{i}
$$

For the egress $\left(\mathcal{E}_{3,4}:=\mathcal{V}\left(\phi_{4}\right) \backslash \mathcal{V}\left(\phi_{3}\right)\right)$, the respective formula is

$$
\Delta F_{3,4}=\sum_{i \in \mathcal{E}_{3,4}} I_{i} A_{i}
$$

From these equations, we see that artifacts may occur. We have to consider two cases: (i) If only the ingress of the accretion stream is used for eclipse mapping: The intensity decrease $\Delta F_{1,2}$ is attributed to all surface elements with $i \in \mathcal{I}_{1,2}$, so if $\mathcal{I}_{1,2}$ contains more than one element, one cannot distinguish between them. This is called aliasing. (ii) If ingress and egress data are available, one can have different sets of simultanously 


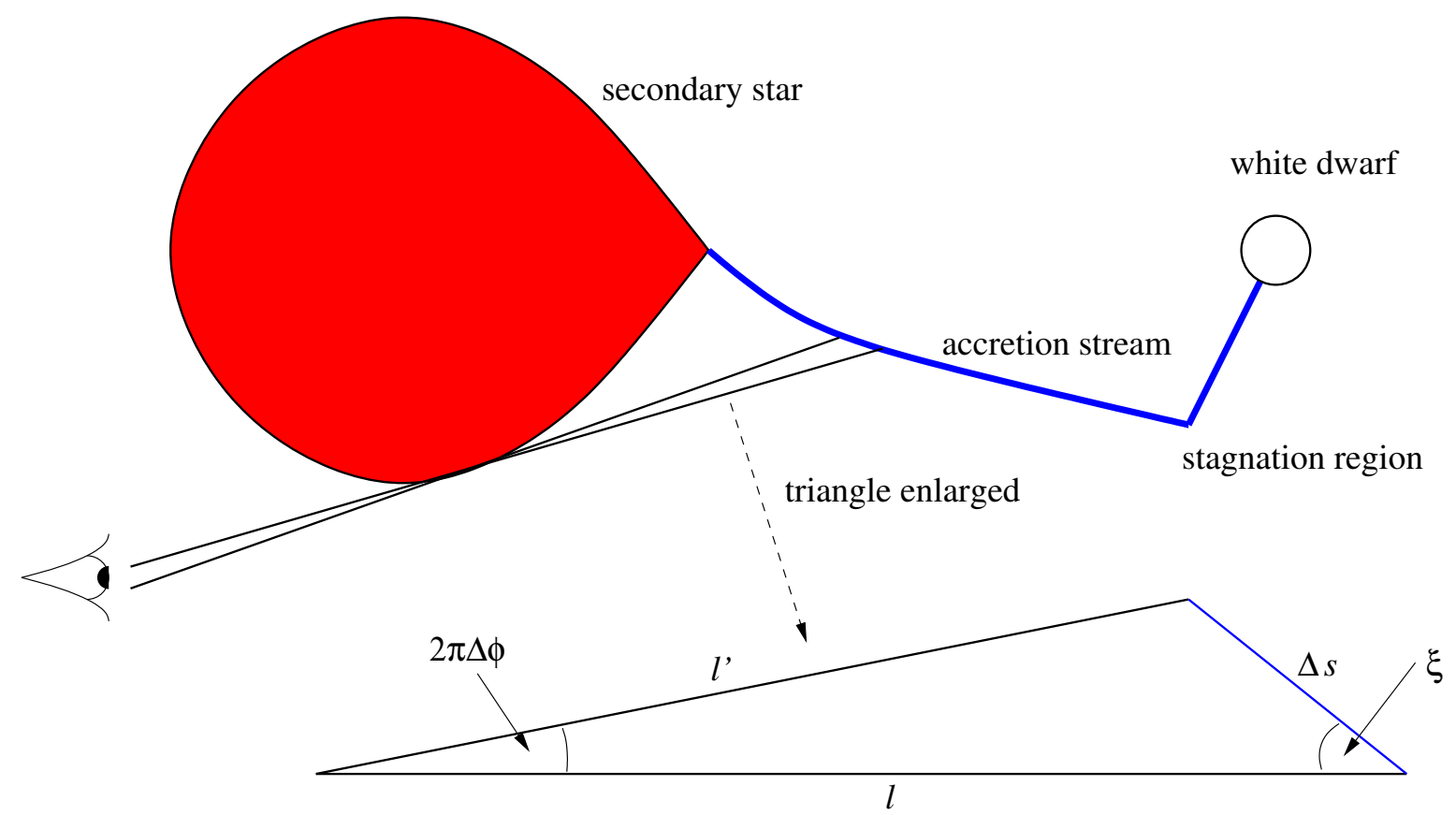

Figure 3.2: Derivation of the smallest possible size of a surface element, $\Delta s$. See Eq. (3.7).

disappearing/reappearing surface elements. $\mathcal{E}_{i, j}$ denominates the surface elements reappearing between the phases $\phi_{i}$ and $\phi_{j}$. If, say, elements $3,4,5 \in \mathcal{I}_{1,2}$ disappear at the same time, we can still distinguish their intensities, if $3 \in \mathcal{E}_{3,4}, 4 \in \mathcal{E}_{4,5}$ and $5 \in \mathcal{E}_{5,6}$. In real polars, this is the case for the surface elements on the ballistic stream and on the magnetically funneled part of the accretion stream, if $\beta \neq 0$ (dipole tilt) and $i<90^{\circ}$ (inclination). Using this, aliasing effects can be reduced in many cases.

To avoid aliasing, one has to choose the size of the surface elements that $\left|\mathcal{I}_{i, i+1}\right|=1$ and $\left|\mathcal{E}_{i, i+1}\right|=1$ for all $i$, that is, one surface elements appears or disappears at a time. How large is such a surface element? The size in direction of the accretion flow $\Delta s$ is the interesting value. As we can see from Fig. 3.2,

$$
\frac{\ell^{\prime}}{\sin \xi}=\frac{\Delta s}{\sin 2 \pi \Delta \phi}
$$

with $\Delta \phi$ the orbital (time-) resolution. Since $\Delta s \ll \ell$, we can use $\ell^{\prime} \approx \ell$. Also, $\ell$ is approximately the distance from the surface element to the center of the secondary star. Since we are interested in the absolute value of $\Delta s$, we then find

$$
\frac{\Delta s}{\ell} \approx \frac{\sin 2 \pi \Delta \phi}{|\sin \xi|}
$$

From Eq. (3.8), we learn that (a) the spacial resolution is highest perpendicular to the line of sight $\left(\xi=90^{\circ}\right)$, (b) the spacial resolution decreases with distance from 
the secondary star and (c) the time resolution transforms nearly linearly into spatial resolution for small $\Delta \phi$, giving a useful resolution of $\Delta s \approx 1 / 100 \cdot a$ for $\Delta \phi \leq 0.001$.

\subsection{1d to quasi-3d: "full-orbit eclipse mapping"}

Full-orbit eclipse mapping (FOEM), which I describe in detail in Chapt. 5 on pp. 45 ff., uses the same type of information as eclipse mapping, but extends the phase coverage over the full orbit. This allows to add a new degree of freedom in the reconstruction of accretion streams in polars. In the eclipse mapping case one typically lets the surface elements extend only in the direction of the accretion flow. Using FOEM, one can try to map intensity structures around the accretion stream as well.

While in eclipse mapping most of the information content comes from the gradually moving "shadow", i.e., the region which is hidden behind the secondary star, one can extend the underlying light curve synthesis and reconstruction method over the full orbit. If one assumes optically thick emission from the structures which are to be reconstructed, the different viewing angles of these structures over the orbit allow for intensity changes. Additionally, besides of the eclipse by the secondary stars, the selfeclipse of emitting regions is important. By self-eclipse is when the emitting parts of a structure rotate away from the view of the observer. Some typical examples of orbital variations of the overall intensity are

- the double-hump light curve seen in emission lines of polars (Chapt. 5). This is mainly a result of the different apparent sizes of the accretion stream over an orbit and can be taken as a direct hint for the optically thick character of the emission lines of the stream,

- the single-hump light curve from the accretion spot in polars (Cropper and Warner, 1986), where the spot on the white dwarf is self-eclipsed,

- the single-hump light curve from an hot spot in quiescent dwarf novae (Wood et al., 1986), which also is self eclipsed.

In principle, for the FOEM method one needs to know the spatial structure of the mapped components in advance. In the interpretation of the resulting maps, these constraints have to be taken into account.

\subsection{2d to 2d: classical Doppler tomography}

The classical Doppler tomography is the method with the closest link to tomography as it is used in medical devices. While in the so-called CT in medicine a single scan 
is a $d^{\prime}=2$ image and the patient is being X-rayed in $n$ different angles ${ }^{2}$, in Doppler tomography the scan is a $d^{\prime}=1$ dimensional image (a high resolution spectrum) taken at $n$ different orbital phases.

\subsubsection{The method}

To understand Doppler tomography, one has to understand the concept of real space and velocity space. In "real space", the coordinate axes are the cartesian spatial coordinates, measured in length units; a position is given as a spatial vector $\vec{r}=\left(r_{x}, r_{y}, r_{z}\right)$. This is the space which we are using when we speak of "position" in the every day context (Fig. 3.3, top).

In "velocity space", the coordinates of a particle are given by its velocity components (Fig. 3.3, bottom). Hence, the axes are labeled with velocity units instead of length units. A position is given as a velocity vector, $\vec{v}=\left(v_{x}, v_{y}, v_{z}\right)$. Which velocity to use? Since velocity space is to help understand observation done from outside the binary system, we use the velocity a particle has as seen from an observer which does not corotate with the orbital components. A possible systemic velocity of the binary system is removed.

As a convention for the orientation of the velocities in velocity space, it was introduced to look at the binary system at the orbital phase $\phi=0.0$, defining the axes that the observer is located at $x=-\infty$. Then we have the velocity of the WD,

$$
\vec{v}_{\text {pri }}=\left(0,-V_{1} \sin i,-V_{1} \cos i\right)
$$

and of the secondary star,

$$
\vec{v}_{\mathrm{sec}}=\left(0,+V_{2} \sin i,+V_{2} \cos i\right)
$$

(Fig. 3.3, top and bottom).

Looking at a close binary star with an imaging instrument like a photographic plate will always show a single point which is spatially unresolved. It will vary in its brightness due to the orbital motion of the components. Hence, the observation leads to a 1-dimensonal dataset (flux over time, $F(\phi)$, where time is translated into orbital phase). We used this type of data in the former section about eclipse mapping and "full-orbit eclipse mapping". But when a close binary is observed with a high resolution spectrograph, one can see the Doppler shift of spectral lines induced by the moving components where the lines are formed.

Let's look at different points of the accretion disk in Fig. 3.3. The point labeled "2" moves to $-x$ and $+y$ with a speed determined by its Kepler velocity. Additionally there is the velocity component given by the system's rotation. Now think of the line

\footnotetext{
${ }^{2}$ The actual imaging is done by a spiralling detector, which is equivalent to the $d^{\prime}=2$-description
} 

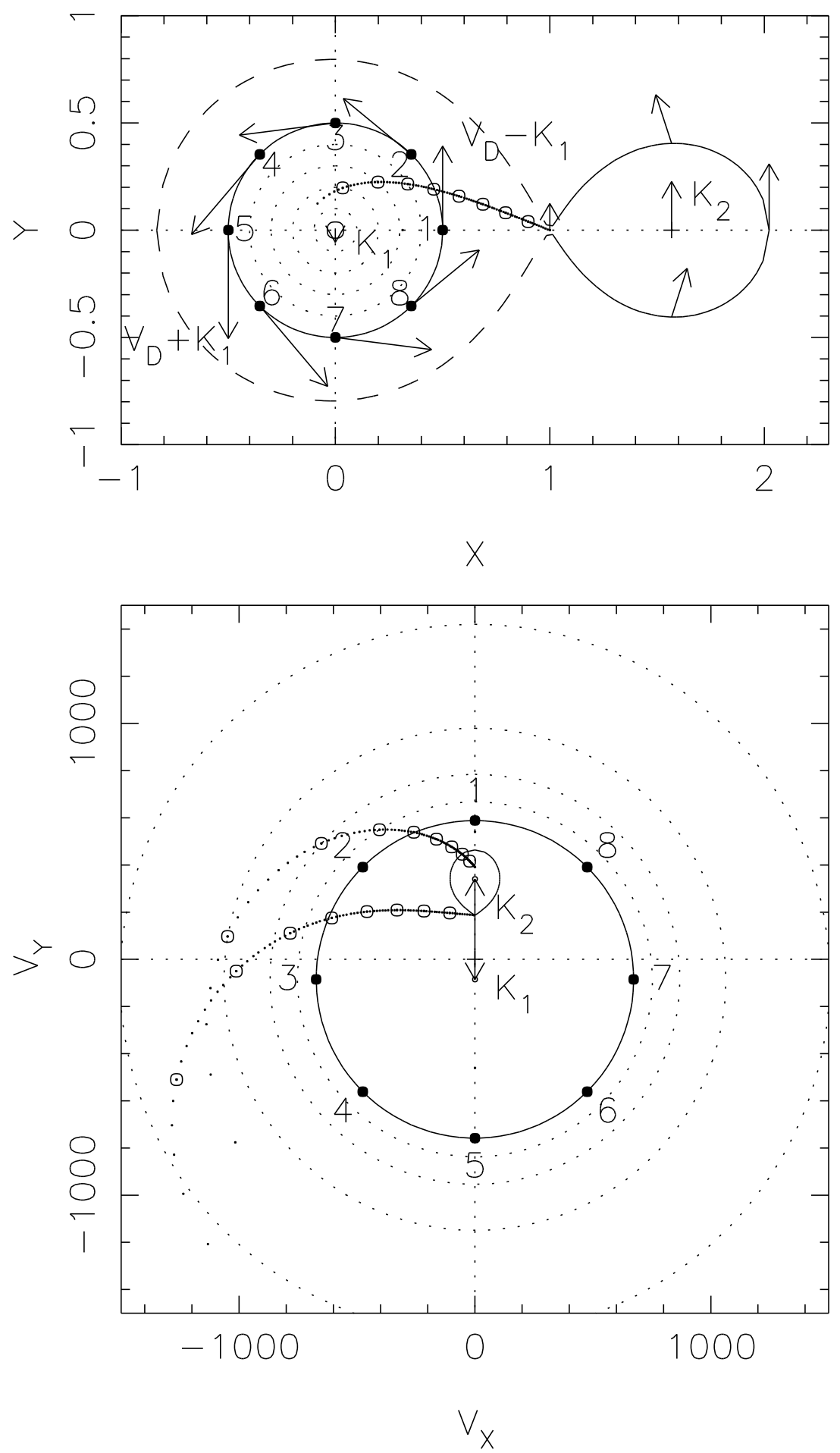

Figure 3.3: Spatial coordinates (top) and velocity coordinates (bottom). See text. (Figures from Keith Horne) 
emission which may occur at the point "2". The observed emission line wavelength from a single line emitting point will wiggle around the rest wavelength sinusoidally:

$$
\begin{array}{rlrl}
\lambda_{\text {obs }}\left(\phi, v_{x}, v_{y}\right) & =\lambda_{0}+\frac{1}{c} \cdot v \cos \left(\phi-\phi_{0}\right) & \text { with } \\
\phi_{0} & =\arcsin \left(v_{y} / v\right)=\arccos \left(v_{x} / v\right) & & \text { and } \\
v & =\sqrt{v_{x}^{2}+v_{y}^{2}} &
\end{array}
$$

Thus, the observed spectrum at a given phase can be calculated from the known velocity space image by integrating the lines with the given wavelength from Eq. 3.10a. This can be illustrated by parallel projection of the velocity space image to a single line with an angle given by the orbital phase.

The understanding of generating single spectra of an object as projection of a known velocity space image brings us to the understanding of the inverse problem. Given is a set of spectra taken at different orbital phases. Then one can generate the velocity space by backprojection of the spectra into the velocity space. In principle, one takes a spectrum, rotates it according to the orbital phase it corresponds to and smears it out over the velocity map perpendicularly to the rotated dispersion direction. To make a mathematically correct transformation ("Radon transform"), the spectra are filtered before the backprojection, hence the method is called "fourier filtered backprojection". Details about the backprojection methods can be found in Deans' Book on the Radon transform (Deans, 1993). Technically, the inversion can also be made using a maximum entropy method (Skilling and Bryan, 1984), which in some cases gives even better results but is slower.

The assumptions for the validity of the Doppler maps are

1. all emitting regions are visible over the full orbit,

2. the flux from any point in the velocity space is constant over the observation interval,

3. the intrinsic shape of the emission line profile can be neglected,

4. all motion is parallel to the orbital plane,

5. the velocity vectors rotate with the orbital motion of the binary system.

Three corrolaries of the assumptions:

- The intensity integrated over the emission line profile is constant over the orbit:

$$
\int_{\text {line }} F(\lambda, \phi) \mathrm{d} \lambda=\int_{\text {line }} F\left(\lambda, \phi^{\prime}\right) \mathrm{d} \lambda \quad \forall \phi, \phi^{\prime}
$$


- The emission line profile is point-symmetric around $(v, \phi)=(0,0)$ :

$$
F(\lambda, \phi)=F\left(\lambda_{0}-\lambda,-\phi\right)
$$

- The integrated line profile is symmetric around zero velocity:

$$
\int_{\text {line }} F(\lambda, \phi) \mathrm{d} \phi=\int_{\text {line }} F(-\lambda, \phi) \mathrm{d} \phi
$$

\subsubsection{Interpretation of Doppler maps}

Now have another look at Fig. 3.3. The points labeled "1" to " 8 " show the behaviour of the outer disk rim. The disk rim in velocity coordinates is turned $90^{\circ}$ to the left from the real space image. Additionally, the disk is not centered around $\vec{v}=(0,0)$, but around the white dwarf's position $\vec{v}=\left(-K_{1}, 0\right)$. This is because the disk rotates around the white dwarf.

The dotted circles describe equally spaced rings in the accretion disk. In velocity coordinates, these rings go from low velocities to ever increasing velocities, because rings in the accretion disk follow Kepler's law:

$$
v_{\text {kep }}(r)=\sqrt{\frac{G M_{1}}{r}}
$$

Hence, rings closer to the white dwarf have higher velocities. A disk is seen inside-out in velocity space.

Note, that the secondary star keeps its Roche shape when transformed into the velocity space, being turned around $90^{\circ}$ just like the accretion disk. The WD is positioned at $\vec{v}=\left(-K_{1}, 0\right)$.

All other structures seen in the Doppler maps are not directly transformable into real space. The strategy to interpret Doppler maps is this: A physical model of the position and velocity of the binary star's components is made. From this, the trajectory of e.g. accretion structures is calculated in the velocity space and overplotted to the Doppler image. Then, the physical model is changed until the "observed" Doppler map is explained to a sufficient level. An example is real space and velocity space display of the accretion stream in Fig. 3.3: The circles from the $L_{1}$-point are located on a ballistic accretion stream, which is very easy to recognize in the real space image (top frame). In the velocity space, the location of the accretion stream is completely different: The velocity in the direction towards the white dwarf $\left(-v_{y}\right)$ dominates at low velocities perpendicular to the line connecting the stars $\left(\right.$ low $\left.+v_{x}\right)$. The second line originating on the back side of the secondary star in the velocity space is given by the Kepler velocities an accretion disk element would have on the referring point on the ballistic 
accretion stream. Even more complicated is the velocity space representation of other structures like magnetically influenced streams (Schwope et al., 1999; Wynn, 2001).

One should also keep in mind, that every velocity perpendicular towards the observation direction is invisible. Hence, a Doppler map is alway restricted to the $v_{x}-v_{y}$-plane. Since the observed $v_{x}-v_{y}$ plane is not neccessarily the orbital plane of the systems (that would only be tha case for $i=90^{\circ}$ ), one has to take into account how out-of-the-plane velocities transform to the observed $v_{x}-v_{y}$-plane at a given inclination.

\section{$3.52 \mathrm{~d}$ to quasi-3d: orbital mapping}

While the Doppler mapping method consists of a direct transformation of the time resolved $d^{\prime}=1$-dimensional images to a $d=2$-dimensional image in the velocity space, the orbital mapping method is a forward synthesis method of a $d=2$ dimensional image on a 3d-grid of surface elements to fit to the same data set as in the Doppler mapping method. Since the resulting map is 2-dimensional, but folded over a 3-dimensional surface structure, I denominate the result of the mapping process a "quasi-3d" map.

Although the kinship to the Doppler mapping method seems to be obvious, orbital mapping is better understood as an enhanced FOEM (Sect. 3.3) method. While in eclipse mapping and FOEM, the aim of the method is to find a map whose transformation into a lightcurve fits to the observed data, in orbital mapping the synthesised data type is a time-resolved spectrum.

Similar to Eq. (3.3), the synthetic spectrum can be described as follows:

$$
F(\lambda, \phi)=\sum_{i \in \mathcal{V}(\phi)} I_{i} C_{i}(\lambda, \phi)
$$

The matrix $A_{i}(\phi)$ in Eq. (3.3) which contained all the physical information in the eclipse mapping and FOEM methods is exchanged by the tensor $C_{i}(\lambda, \phi)$. This tensor holds the information of how much the surface element $i$ contributes to the observed flux at the wavelength $\lambda$ at the orbital phase $\phi$. If only one wavelength interval is observed, $C_{i}(\lambda, \phi)$ in fact is identical to $A_{i}(\phi)$ in Eq. (3.3).

The mapping process now again is (1) calculate the model spectrum $F_{\text {model }}(\lambda, \phi)$ for a given intensity vector $I_{i}$ using Eq. (3.15), (2) compare $F_{\text {model }}(\lambda, \phi)$ to the observed data, $F(\lambda, \phi),(3)$ change $I_{i}$ and restart at (1), using additional constraints to get a smooth map.

A more detailed description is found in Chapt. 6. 


\section{Chapter 4}

\section{Eclipse Mapping}

This chapter summarizes the work done on classical Eclipse mapping. It is based on the article "Eclipse mapping of the accretion stream in UZ Fornacis" (Kube et al., 2000).

\subsection{Introduction}

Polars (also called AM Her systems after their prototype) are CVs with a WD, that has a very high magnetic field $(7 \ldots 230 \mathrm{MG})$. During their development, the rotation of the WDs has synchronized with the orbital motion, hence they are phase locked.

The magnetic field is so strong that the formation of an accretion disk is prevented: At some point, the ram pressure of the in-falling material is lower than the magnetic pressure on electrically charged particles. The single plasma particles are forced on circular orbits around a magnetic field line, which leads to a stream movement along that field line (Fig. 4.1). The exact position of the coupling region (sometimes also called stagnation region, SR) is defined by the equality of plasma ram pressure and magnetic pressure, hence depends on the magnetic field strength, the accretion rate and the size of the accretion stream. Fortunately, one can infer the position of the stagnation region in eclipsing systems by a UV and X-Ray dip, produced by the magnetically funneled stream, which absorps the hard radiation from the impact region ("hot spot") on the white dwarf. This dip is positioned in phase at around $\phi=0.85 \ldots 0.95$.

The accretion rate in polars undergoes drastic changes (Hessman et al., 2000), leading to low states when the accretion stream with its line emission is invisible and high states where the accretion stream dominates the spectrum. The low state can be used to spectroscopically determine the magnetic field strength of the hot spots, where anisotropic cyclotron radiation is produced.

From the point of view of the mapping process, it is important to know that the accretion stream gives a restriction of the region where emission can come from in 


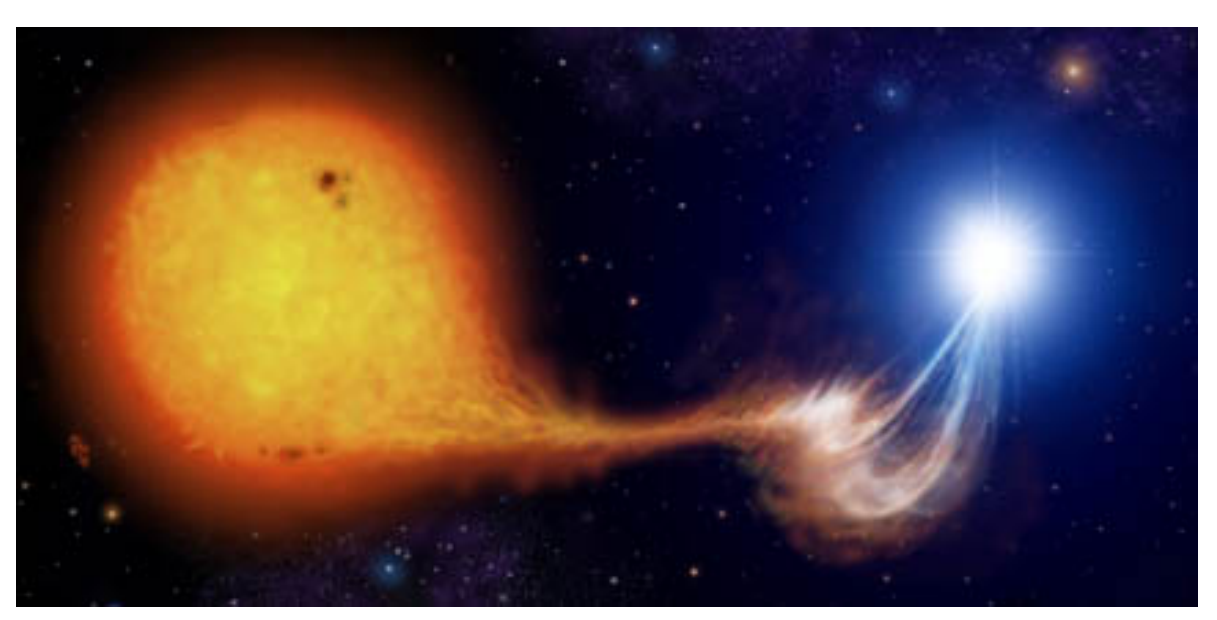

Figure 4.1: Artist's view of a polar (Mark Garlick).

principle. Other than in disk systems, a simple $2 \mathrm{~d}$ grid of an symmetric surface (the disk) around the white dwarf can not be used. Instead, the geometry with the free falling (ballistic) accretion stream, the coupling region and the magnetically funneled stream ("dipole stream") has to be modeled as a grid of surface elements.

In systems with an inclination $i \gtrsim 70^{\circ}$, the secondary star gradually eclipses the accretion stream during the inferior conjunction. Using tomographical methods, it is - in principle - possible to reconstruct the surface brightness distribution on the accretion stream from time resolved observations. This method has been successfully applied to accretion discs in non-magnetic CVs ("eclipse mapping", Horne (1985)). In this chapter, I present tests and a first application of a new eclipse mapping code, which allows the reconstruction of the intensity distribution on the accretion stream in magnetic CVs.

Similar attempts to map accretion streams in polars have been investigated by Hakala (1995) and Vrielmann and Schwope (1999) for HU Aquarii. An improved version of Hakala's (1995) method has been presented by Harrop-Allin et al. (1999b) with application to real data for the system HU Aquarii (Harrop-Allin et al., 1999a). A drawback of all these approaches is that they only consider the eclipse of the accretion stream by the secondary star. In reality, the geometry may be more complicated: the far side of the magnetically coupled stream may eclipse stream elements close to the WD, as well as the hot accretion spot on the WD itself. The latter effect is commonly observed as a dip in the soft X-ray light curves prior to the eclipse (e.g. Sirk and Howell, 1998). The stream-stream eclipse may be detected in data which are dominated by emission from the accretion stream, e.g. in the light curves of high-excitation emission lines where the secondary contributes only little to the line flux.

Here, I describe a new accretion stream eclipse mapping method, using a $3 \mathrm{~d}$ code which can handle the full complexity of the geometry together with an evolution strategy as 

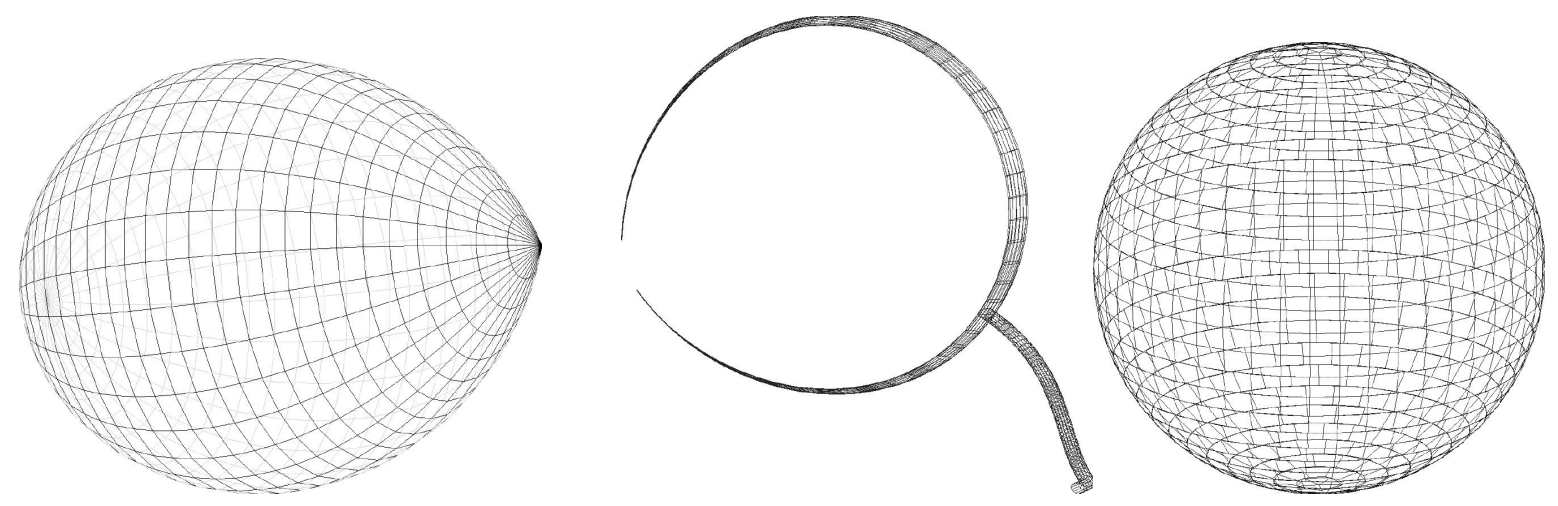

Figure 4.2: $3 \mathrm{~d}$ grid of the secondary star (left), the accretion stream (middle), and the white dwarf (right). Not to scale!

fit algorithm. I present extensive tests of the method and map as a first application to real data the accretion stream in UZ For emission of C IV $\lambda 1550$.

\subsection{The $3 \mathrm{~d}$ cataclysmic variable model}

The computer code CVMOD generates $N$ small surface elements (convex quadrangles, some of which are degenerated to triangles), which represent the surfaces of the individual components of the CV (WD, secondary, accretion stream) in three-dimensional space (Fig. 4.2). Using simple rotation algorithms, the position of each surface element $i=1 \ldots N$ can be computed for a given orbital phase $\phi$.

The white dwarf is modeled as an approximated sphere, using surface elements of nearly constant area (Gänsicke et al., 1998). The secondary star is assumed to fill its Roche volume. Here, the surface elements are choosen in such a way that their boundaries align with longitude and latitude circles of the Roche surface, taking the $L_{1}$-point as the origin.

The surface of the accretion stream is generated in two parts, (a) the ballistic part from $L_{1}$ to SR, and (b) the dipole-part from SR to the surface of the white dwarf.

(a) For the ballistic part of the stream, I use single-particle trajectories. The equations of motion in the corotating frame are given by

$$
\begin{aligned}
\ddot{x} & =+\mu \frac{x-x_{1}}{r_{1}^{3}}-(1-\mu) \frac{x-x_{2}}{r_{2}^{3}}+2 \dot{y}+x \\
\ddot{y} & =-\mu \frac{y}{r_{1}^{3}}-(1-\mu) \frac{y}{r_{2}^{3}}-2 \dot{x}+y \\
\ddot{z} & =-\mu \frac{z}{r_{1}^{3}}-(1-\mu) \frac{z}{r_{2}^{3}}
\end{aligned}
$$


Eq. (4.3) has been added to Flannery's (1975) set of two-dimensional equations. $\mu=$ $\left(M_{1}+M_{2}\right) / M_{1}$ is the inverse mass fraction of the white dwarf, $r_{1}$ and $r_{2}$ are the distances from the point $(x, y, z)$ to the white dwarf and the secondary, respectively, in units of the orbital separation $a$. The coordinate origin is at the center of gravity, the $x$-axis is along the lines connecting the centers of the stars, the system rotates with the angular frequency $\omega=2 \pi / P$ around to the $z$-axis. The velocity $\vec{v}=(\dot{x}, \dot{y}, \dot{z})$ is given in units of $a \omega, \overrightarrow{v_{0}}=\left(\dot{x}_{0}, \dot{y}_{0}, \dot{z}_{0}\right)$ is the initial velocity in the $L_{1}$ point.

If $\dot{z}_{0}=0$, the trajectories resulting from the numerical integration of Eqs. (4.1) - (4.3) are restricted to the orbital plane. However, calculating single-particle trajectories with different initial velocity directions (allowing also $\dot{z}_{0} \neq 0$ ) shows that there is a region approximately one third of the way downstream from $L_{1}$ to SR where all trajectories pass within very small separations, corresponding to a striction of the accretion stream.

I define a $3 \mathrm{~d}$ version of the stream as a tube with a circular cross section with radius $r_{\text {Tube }}=5 \times 10^{8} \mathrm{~cm}$ centred on the single-particle trajectory for $\dot{x}=10 \mathrm{~km} \mathrm{~s}^{-1}, \dot{y}=\dot{z}=0$.

(b) When the matter reaches SR, I switch from a ballistic single-particle trajectory to a magnetically forced dipole geometry. The central trajectory is generated using the dipole formula $r=r_{0} \sin ^{2} \alpha$, where $\alpha$ is the angle between the dipole axis and the position of the particle $(r, \varphi, \alpha)$. This can be interpreted as the magnetic field line $F$ passing through the stagnation point SR and the hot spots on the WD. Knowing $F$, I assume a circular cross section with the radius $r_{S R}=r_{\text {Tube }}=5 \times 10^{8} \mathrm{~cm}$ for the region where the dipole intersects the ballistic stream. This cross section is subject to transformation as $\alpha$ changes. Thus, the cross section of the stream is no longer constant in space but bounded everywhere by the same magnetic field lines.

The accretion stream model involves several assumptions: (1) The cross section of the stream itself is to some extent arbitrary because I consider it to be - for the data actually used, see below - essentially a line source. (2) The neglect of the magnetic drag (King, 1993; Wynn and King, 1995) on the ballistic part of the stream and the neglect of deformation of the dipole field may cause the model stream to deviate in space from the true stream trajectory. While, in fact, the location of SR may fluctuate with accretion rate (as does the location of the Earth's magnetopause), the evidence for a sharp soft X-ray absorption dip caused by the stream suggest that SR does not wander about on time scales short compared to the orbital period. (3) The abrupt switch-over from the ballistic to the dipole part of the stream may not describe the physics of SR correctly (see Sect. 5.3). This discrepancy, however, does not seriously affect the results, because the eclipse tomography is sensitive primarily to displacements in the times of ingress and egress of SR which are constrained by the absorption dip in the UV continuum (and, in principle, in soft X-rays). The $\approx 5$ sec time bins of the observed light curves correspond to $\approx 10^{8} \mathrm{~cm}$ in space at SR. Hence, the code is insensitive to structure on a smaller scale. In fact, the smallest resolved structures are much larger because of the noise level of the data. While this approach clearly involves several 
approximations, it is tailored to the desired aim of mapping the accretion stream from the information obtained from an emission line light curve.

In this code, I restrict the possible brightness distribution to being equal for each stream segment which consists of 16 surface elements forming a section of the tube-like stream, i.e. there is no intensity variation around the stream. For the current data, this is no serious drawback, because only observations covering a small phase interval around the eclipse are used. The results refer, therefore, to the stream brightness as seen from the secondary. From the present observations one can not infer how the fraction of the stream illuminated by the X-ray/UV spot on the WD looks like. The required extension of the computer code, allowing for brightness variation around the stream, is presented in Chapt. 5, starting at p. 45.

\subsection{Light curve fitting}

The basic idea of this eclipse mapping algorithm is to reconstruct the intensity distribution on the accretion stream by comparing and fitting a synthetic light curve to an observed one. The comparison between these light curves is done with a $\chi^{2}$ minimization, which is modified by means of a maximum entropy method. Sect. 4.3.1 describes the light curve generation, Sect. 4.3.2 the maximum entropy method, and Sect. 4.3.3 the actual fitting algorithm.

\subsubsection{Light curve generation}

In order to generate a light curve from the $3 \mathrm{~d}$ model, it is neccessary to know which surface elements $i$ are visible at a given phase $\phi$. I designate the set of visible surfaces $\mathcal{V}(\phi)$.

In general, each of the three components (WD, secondary, accretion stream) may eclipse (parts of) the other two, and the accretion stream may partially eclipse itself. This is a typical hidden surface problem. However, in contrast to the widespread computer graphics algorithms which work in the image space of the selected output device (e.g. a screen or a printer), and which provide the information 'pixel $j$ shows surface $i$ ', one needs to work in the object space, answering the question 'is surface $i$ visible at phase $\phi$ ?'. For a recent review on object space algorithms see Dorward (1994). Unfortunately, there is no readily available algorithm which fits this needs, thus I use a self-designed 3 d object-space hidden-surface algorithm. Let $N$ be the number of surface elements of the 3d model. According to Dorward (1994), the time $T$ needed to perform an object space visibility analysis goes like $T \propto N \log N \ldots N^{2}$. My algorithm performs its task in $T \propto N^{1.5 \ldots 1.8}$, with the faster results during the eclipse of the system. It is 
obviously necessary to optimize the number of surface elements in order to minimize the computation time without getting too coarse a $3 \mathrm{~d}$ grid.

Once $\mathcal{V}(\phi)$ has been determined, the angles between the surface normals of $i \in \mathcal{V}(\phi)$ and the line of sight, and the projected areas $A_{i}(\phi)$ of $i \in \mathcal{V}(\phi)$ are computed. Designating the intensity of the surface element $i$ at the wavelength $\lambda$ with $I_{i, \lambda}$, the observed flux $F_{\lambda}(\phi)$ is

$$
F_{\lambda}(\phi)=\sum_{i \in \mathcal{V}(\phi)} I_{i, \lambda} A_{i}(\phi)
$$

Here, two important assumptions are made: (a) the emission from all surface elements is optically thick, and (b) the emission is isotropic, i.e. there is no limb darkening in addition to the foreshortening of the projected area of the surface elements. The computation of a synthetic light curve is straightforward. It suffices to compute $F_{\lambda}(\phi)$ for the desired set of orbital phases.

While the above mentioned algorithm can produce light curves for all three components, the WD, the secondary, and the accretion stream, I constrain in the following the computations of light curves to emission from the accretion stream only. Therefore, I treat the white dwarf and the secondary star as dark opaque objects, screening the accretion stream.

\subsubsection{Constraining the problem: maximizing the entropy}

In the eclipse mapping analysis, the number of free parameters, i.e. the intensity of the $N$ surface elements, is typically much larger than the number of observed data points. Therefore, one has to reduce the degrees of freedom in the fit algorithm in a sensible way. An approach which has proved successful for accretion discs is the maximum entropy method (Horne, 1985). The basic idea is to define an image entropy $S$ which has to be maximized, while the deviation between synthetic and observed light curve, usually measured by $\chi^{2} / n$, is minimized ( $n$ is the number of phase steps or data points). Let $D_{i}$ be

$$
D_{i}=\frac{\sum_{j=1}^{N} I_{j} \exp \left(-\frac{\left(\vec{r}_{i}-\vec{r}_{j}\right)^{2}}{2 \Delta^{2}}\right)}{\sum_{j=1}^{N} \exp \left(-\frac{\left(\vec{r}_{i}-\vec{r}_{j}\right)^{2}}{2 \Delta^{2}}\right)}
$$

the default image for the surface element $i$. Then the entropy is given by

$$
S=\frac{\sum_{i=1}^{N} I_{i}\left(\ln \frac{I_{i}}{D_{i}}-1\right)}{\sum_{i=1}^{N} I_{i}}
$$


In Eq. (4.5), $\vec{r}_{i}$ and $\vec{r}_{j}$ are the positions of the surface elements $i$ and $j . \Delta$ determines the range of the default image in Eq. (4.5), which is a convolution of the actual image with a Gaussian with the $\sigma$-width of $\Delta$. Hence, the entropy measures the deviation of the actual image from the default image. An ideal entropic image (with no contrast at all) has $S=1$. I use $\Delta=1 \times 10^{9} \mathrm{~cm} \approx 0.02 a$ for the test calculations and for the application to UZ For.

The quality of an intensity map is given as

$$
\mathcal{Q}=\chi^{2} / n-\lambda S
$$

where $\lambda$ is chosen in the order of 1 . Aim of the fit algorithm is to minimize $\mathcal{Q}$.

\subsubsection{The fitting algorithm: evolution strategy}

The accretion stream model involves approximately 250 parameters, which are the intensities of the surface elements. This large number is not the number of the degrees of freedom, which is difficult to define in a maximum entropy strategy. A suitable method to find a parameter optimum with a least $\chi^{2}$ and a maximum entropy value is a simplified imitiation of biological evolution, commonly referred to as 'evolution strategy' (Rechenberg, 1994) or - slightly different - as 'genetic algorithm' (Charbonneau, 1995). The intensity information of the $i$ surface elements is stored in the intensity vector $\vec{I}$. Initially, I choose $I_{i}=1$ for all $i$.

From this parent intensity map, a number of offsprings is created with $I_{i}$ randomly changed by a small amount, the so-called mutation. For all offsprings, the quality $\mathcal{Q}$ is calculated. The best offspring is selected to be the parent of the next generation. An important feature of the evolution strategy is that the amount of mutation itself is also being evolved just as if it were part of the parameter vector. I utilize the C-program library evoC developed by K. Trint and U. Utecht from the Technische Universität Berlin, which handles all the important steps (offspring generation, selection, stepwidth control).

In contrast to the classical maximum entropy optimisation (Skilling and Bryan, 1984), the evolution strategy does not offer a quality parameter that indicates how close the best-fit solution is to the global optimum. In order to test the stability of the method, I run the fit several times starting from randomly distributed maps. All runs converge to very similar intensity distributions $\vec{I}$ (see also Figs. 4.8 and 4.10 ). This type of test is common in evolution strategy or genetic algorithms (e.g. Hakala 1995). Even though this approach is not a statistically 'clean' test, it leaves to conclude that one finds the global optimum.

Fastest convergence is achieved with 40 to 100 offsprings in each generation. Finding a good fit $\left(\chi^{2} / n\right)$ takes only on tenth to one fifth of the total computation time, the 
Table 4.1: System geometry of the imaginary system IM Sys

\begin{tabular}{ll}
\hline \hline mass ratio & $Q=M_{1} / M_{2}=4$ \\
total mass & $M=M_{1}+M_{2}=0.9 M_{\odot}$ \\
orbital period & $P=100 \mathrm{~min}$ \\
inclination & $i=88^{\circ}$ \\
dipole tilt & $\beta=20^{\circ}$ \\
dipole azimuth & $\Psi=35^{\circ}$ \\
angle to SR & $\Psi_{S}=35^{\circ}$ \\
\hline \hline
\end{tabular}

remaining iterations are needed to improve the smoothness of the intensity map, i.e. to maximize $S$.

\subsection{Test calculations}

To test the quality and the limits of the method, I produce synthetical test light curves with different noise levels $(\mathrm{S} / \mathrm{N}=\infty, 50,20,10,4)$. Then I try to reproduce the initial intensity distribution on the stream from the synthetic data. Two tests with different intensity distributions are performed. For both tests, the geometry of the imaginary system IM Sys is chosen as shown in Tab. 4.1. The phase coverage is $\phi=0.865 \ldots 1.070$ with 308 equidistant steps, which is identical to the real HST data of UZ For used below for a first application.

Additionally, I test the algorithm with a full-orbit light curve with $\mathrm{S} / \mathrm{N}=10$ to demonstrate its capabilities if more than just the ingress information for each stream section is available.

\subsubsection{One bright region near SR}

In the first test, I set the stream brightness to 1 on the whole stream except for a small region near the stagnation point SR, where the intensity is set to 10. For this intensity distribution, I show the theoretical light curve in Fig. 4.3. One clearly sees the fast ingress of the small bright region at phase $\phi=0.975$, whereas the egress occurs beyond $\phi=1.07$ and is not covered as in the real data of UZ For.

The initial intensity map and the reconstructed map with the different noise levels are shown in Figs. 4.3 and 4.4. The reconstruction of one bright region near the stagnation point is achieved with no artifacts, not too much smearing and little noise for $\mathrm{S} / \mathrm{N} \geqslant 10$. Even with $\mathrm{S} / \mathrm{N}=4$, a reasonable reconstruction can be obtained, but with artifacts: 


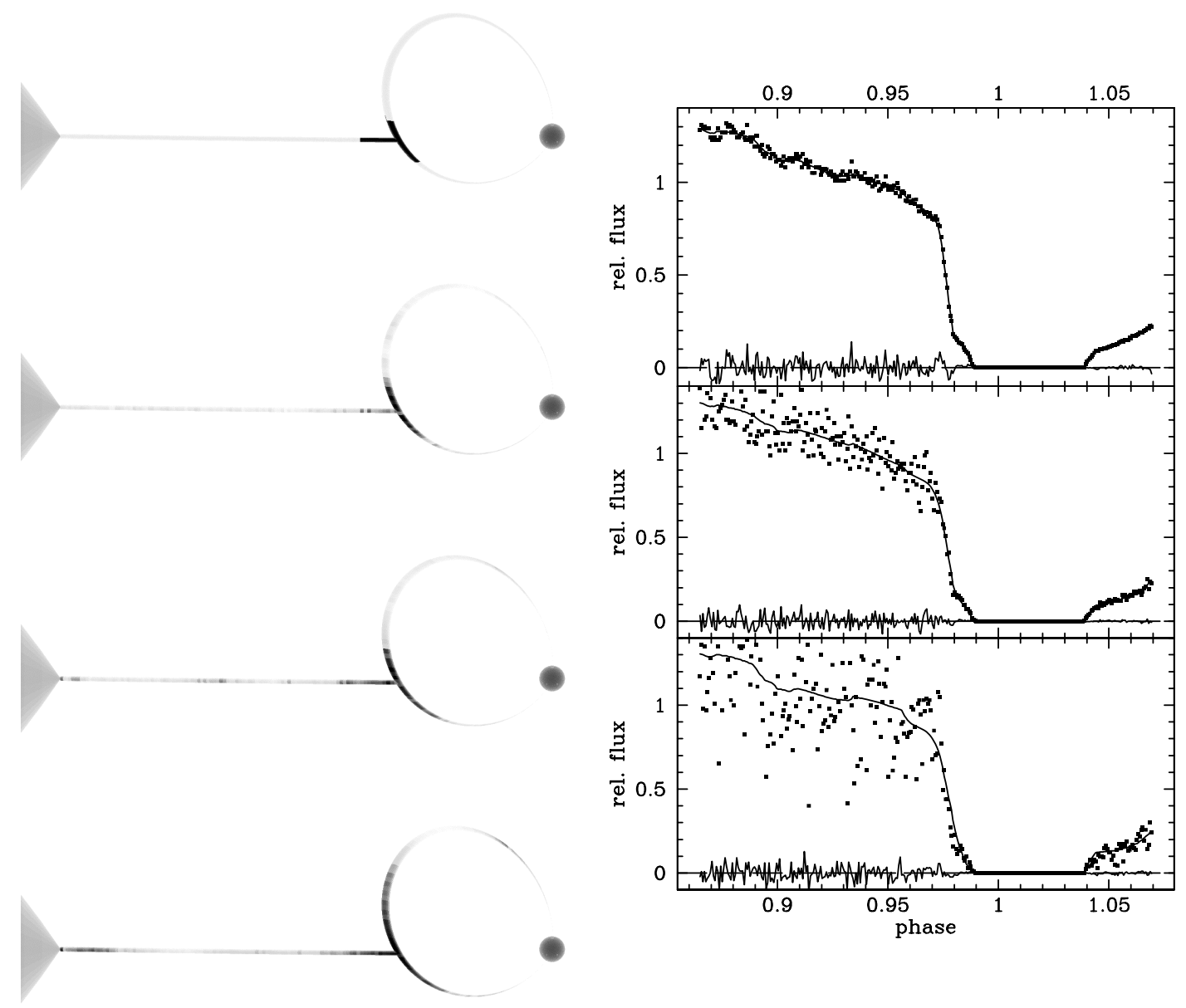

Figure 4.3: Left: Maps of the synthetic stream and its reconstructions. From top to bottom: Input data, reconstructions with $\mathrm{S} / \mathrm{N}=50,10,4$. Right: Synthetic light curve of an accretion stream which is bright around the stagnation region. Different levels of artifical noise are added: $\mathrm{S} / \mathrm{N}=50,10,4$. The reconstructed light curves are shown as solid lines. The residuals are normalized so that the standard deviation $\sigma$ is 0.1 in the relative flux units.

The ballistic stream appears bright near the $L_{1}$-point, and an additional bright region appears on the dipole stream near the northern accretion pole.

\subsubsection{Three bright regions on the ballistic stream}

In the second test I assume a rather unphysical intensity distribution with the aim to test the spatial resolution of this mapping method: The ballistic part of the accretion stream between $L_{1}$ and SR is divided into 5 sections of equal length. Alternately, the intensity on these sections is set alternatingly to 10 and to 1, producing a 'zebra'- 

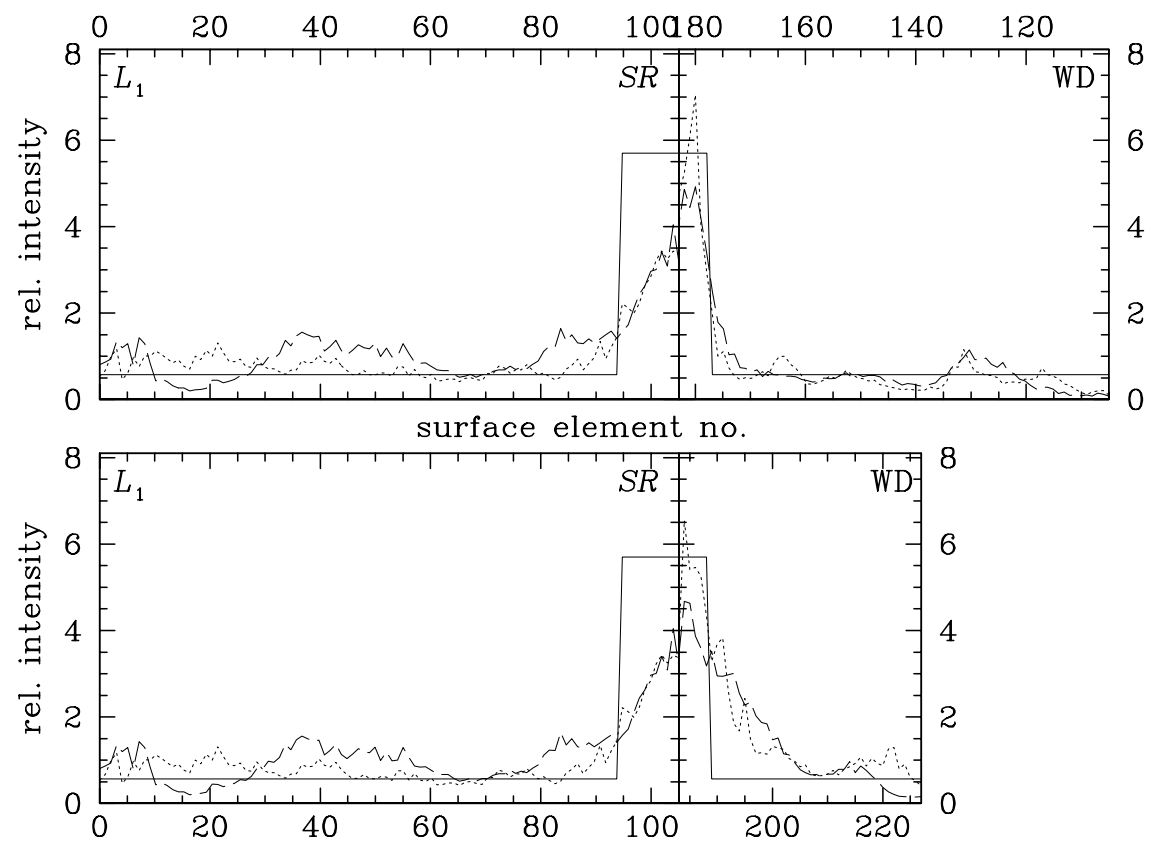

Figure 4.4: Plot of the reconstruction of the synthetic stream with a bright region around SR. Solid line: Input distribution, dotted line: reconstruction with $\mathrm{S} / \mathrm{N}=50$, dashed line: reconstruction with $\mathrm{S} / \mathrm{N}=10$. In the left panels (surface elements no. 0 to 104), the intensities of the surface elements on the ballistic stream are shown. In the upper right panel, the northern magnetic stream is to be found (surface elements no. 105 to 182), in the lower right panel the southern magnetic stream (surface elements no. 183 to 227).

Table 4.2: Quality and number of iterations for the test calculations.

\begin{tabular}{clclc}
\hline \hline S/N & $\chi^{2} / n$ & $S$ & $\chi^{2} / n$ & $S$ \\
\hline & bright region near SR & \multicolumn{2}{l}{ three } & bright regions \\
$\infty$ & 0.015 & 0.982 & 0.018 & 0.938 \\
50 & 0.173 & 0.947 & 0.284 & 0.960 \\
20 & 0.593 & 0.968 & 0.668 & 0.953 \\
10 & 0.886 & 0.971 & 0.924 & 0.983 \\
4 & 1.038 & 0.980 & 1.038 & 0.984 \\
\hline \hline
\end{tabular}

like pattern. The intensity on the magnetically funneled stream is set to $I=1$. The synthetic light curve for this intensity distribution differs strongly from that in out first test (Fig. 4.5). Instead of one sharp step in the light curve, there are now - as expected - three steps during the ingress and three during the egress (also not visible in the selected phase interval). 


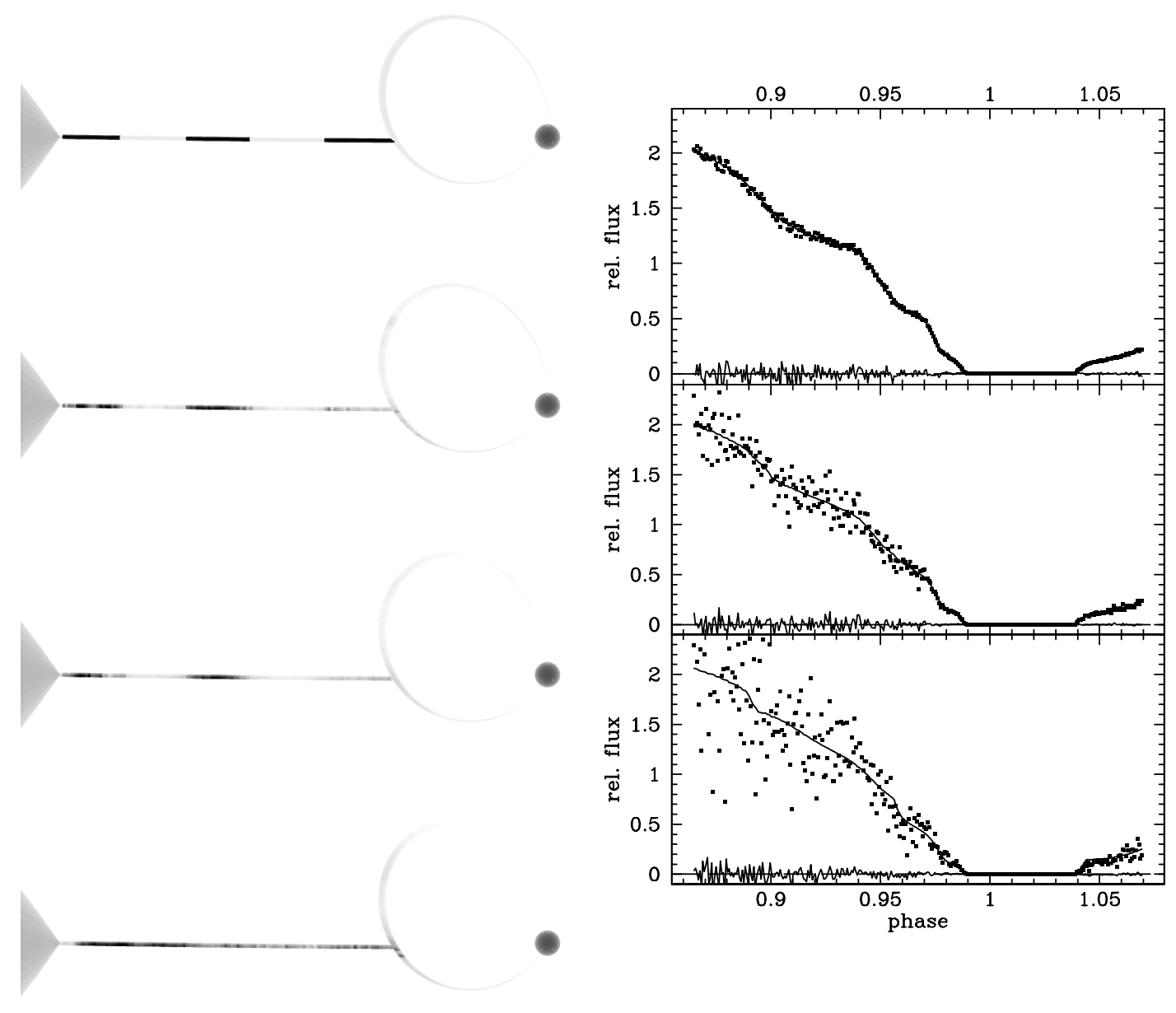

Figure 4.5: Left: Maps of the synthetic stream and its reconstructions. From top to bottom: Input data, reconstructions with $\mathrm{S} / \mathrm{N}=50,10,4$. Right: Synthetic light curve of an accretion stream with three bright parts on the ballistic stream. Different levels of artifical noise are added: $\mathrm{S} / \mathrm{N}=50,10,4$. The reconstructed light curves are shown as solid lines. The residuals are normalized so that the standard deviation $\sigma$ is at 0.1 in the relative flux.

In Figs. 4.5 and 4.6 the input and the resulting maps for the 'zebra'-test are show. As long as $\mathrm{S} / \mathrm{N} \geqslant 10$, my algorithm achieves, as in the first test, a good reconstruction of the input map. The bright regions next to SR appear darker than in the original map, since the intensity from that region is spread over the last part of the ballistic stream and the neighbouring parts of the dipole stream, which dissapear behind the limb of the secondary star nearly simultaneously during ingress.

Comparing the two tests for $\mathrm{S} / \mathrm{N}=4$, one finds that it is still possible to distinguish between the two different initial intensity distributions (one bright region and 'zebra') even with such a noisy signal. 


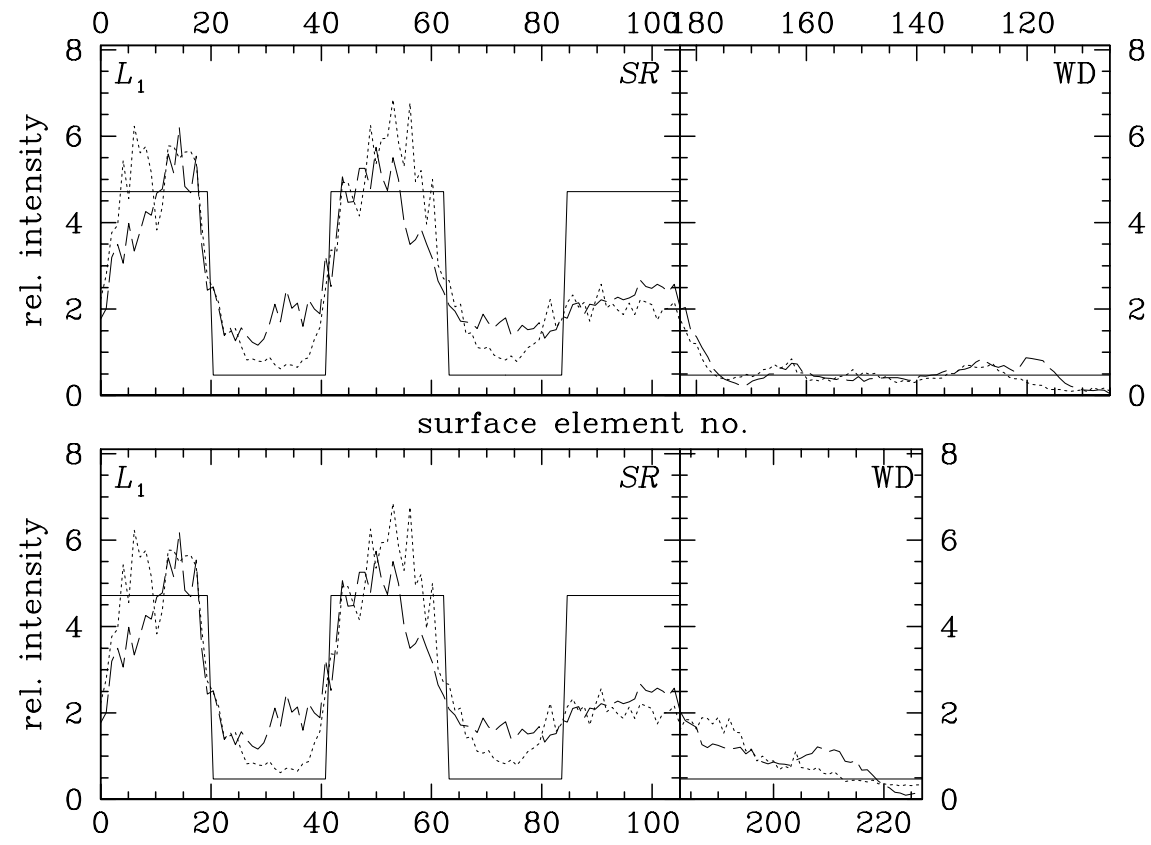

Figure 4.6: Plot of the reconstruction of the synthetic stream with three bright regions on the ballistic stream. Solid line: Input distribution, dotted line: reconstruction with $\mathrm{S} / \mathrm{N}=50$, dashed line: reconstruction with $\mathrm{S} / \mathrm{N}=10$.

In Tab. 4.2, the quality parameters of the fits are listed. The increase of $\chi^{2} / n$ from $\approx 0$ to $\approx 1$ with the decrease of $\mathrm{S} / \mathrm{N}$ shows once more how well the fit algorithm works: For the light curve with no noise, the ideal light curve has - by definition $-\chi^{2}=0$. As soon as noise is added, even the input light curve has a $\chi^{2} / n \approx 1$, which follows from the definition of $\chi^{2}$ if $\sigma$ is the real standard deviation of the data set. For the calculation of $\chi^{2}$, one needs a sensibly chosen standard deviation $\sigma$. Since the light curves of polars show pronounced flickering, I calculate an approximate $\sigma$ for the noisy data sets (synthetic and measured) by comparing the data with a running mean over 10 phase steps. The running mean has, thus, a reduced $\chi^{2}$ of 1 . Since the best fit to artificial data with low synthetic noise levels does better than the running mean, I obtain $\chi^{2} / n<1$ for these light curves.

The entropy $S$ of the images is always close to 1 . An ideal entropic image would have $S=1$, the actual values of $S=0.94 \ldots 0.98$ come very close to that. One would not expect $S=1$, since that would imply that there is no variation in the image at all, i.e., all surface elements have the same brightness. The trend to higher $S$ with lower noise is a result of the weighting of $S$ with respect to $\chi^{2} / n$ in the one-dimensional quality parameter $\mathcal{Q}$ (Eq. 4.7). For the test calculations, I have chosen $\lambda=1$ constant for all noise levels. This emphasizes the reduction of $\chi^{2}$ for low noise levels and the smoothing for high noise levels. 
The tests show that it is possible to detect structures in the brightness distribution on the accretion stream with a size of $\approx 1 \ldots 2 R_{1}$ from data with phase coverage, phase resolution and noise level similar to that of the HST data set for UZ For (see Sect. 4.5.2).

\subsection{Eclipse mapping of UZ For}

As an application for the eclipse mapping method I chose the polar UZ For, which is sufficiently analyzed to allow a good geometrical modeling of the system. I used publicly available UV data from the HST archive at the STScI.

\subsubsection{System Geometry of UZ For}

UZ For has been identified as a polar in 1988 (Berriman and Smith, 1988; Beuermann et al., 1988; Osborne et al., 1988). Cyclotron radiation from a region with $B=53 \mathrm{MG}$ has been reported by Schwope et al. (1990) and Rousseau et al. (1996). The first mass estimates for the WD were rather high, $M_{1}=1.09 \pm 0.01 M_{\odot}$ and $M_{1}>0.93 M_{\odot}$ (Hameury et al., 1988; Beuermann et al., 1988), but Bailey and Cropper (1991) and Schwope et al. (1997b) derived significantly smaller masses, $0.61 M_{\odot}<M_{1}<0.79 M_{\odot}$ and $M_{1}=0.75 M_{\odot}$, respectively. I use reliable system parameters from Bailey and Cropper (1991), $q=M_{1} / M_{2}=5, M=M_{1}+M_{2}=0.85 M_{\odot}, i=81^{\circ}, P=126.5 \mathrm{~min}$. The optical light curve in Bailey (1995) shows two eclipse steps which are interpreted as the signature of hot spots near both magnetic poles of the WD, consecutively dissapearing behind the limb of the secondary star. From that light curve I measure the timing of the eclipse events with an accuracy of $\Delta \phi=5 \cdot 10^{-4}$. The ingress of the spot on the lower hemisphere occurs at $\phi=0.9685$, its egress at $\phi=1.0310$. For the spot on the upper hemisphere, ingress is at $\phi=0.9725$ and egress at $\phi=1.0260$.

To describe the spatial position of the dipole field line along which the matter is accreted, three angles are needed: The colatitude or tilt of the dipole axis $\beta$, the longitude of the dipole axis $\Psi$, and the longitude of the stagnation region $\Psi_{S}$. Here, longitude is the angle between the secondary star and the respective point as seen from the centre of the white dwarf. With the choice of $\beta, \Psi$, and $\Psi_{S}$, it is possible to reproduce the ingress and egress of the two hot spots as well as the dip at phase $\phi=0.9$. A summary of the main system parameters used in this analysis is given in Tab. 4.3.

Having the correct geometry of the accretion stream is crucial for generating the correct reconstruction of the emission regions. As shown by Kube et al. (1999) especially the geometry of the dipole stream is sensitive to changes in $\Psi, \Psi_{S}$, and $\beta$. For UZ For, the geometry of the dipole stream is relatively well constrained from the observed ingress and egress of both hot spots on the white dwarf (Bailey, 1995). Fitting the UZ For light 
Table 4.3: System geometry of UZ Fornacis

\begin{tabular}{ll}
\hline \hline mass ratio & $Q=M_{1} / M_{2}=5$ \\
total mass & $M=M_{1}+M_{2}=0.85 M_{\odot}$ \\
orbital period & $P=126.526229 \mathrm{~min}$ \\
orbital separation & $a=5.49 \times 10^{10} \mathrm{~cm}$ \\
inclination & $i=81^{\circ}$ \\
radius of WD & $R_{1}=7.53 \times 10^{8} \mathrm{~cm}$ \\
\hline dipole tilt & $\beta=12^{\circ}$ \\
dipole azimuth & $\Psi=5^{\circ}$ \\
azimuth of stagnation region & $\Psi_{S}=34^{\circ}$ \\
'radius' of ballistic stream & $r_{S}=5 \times 10^{8} \mathrm{~cm}$ \\
\hline \hline
\end{tabular}

curves system geometries that differ within the estimated error range of less than five degrees does, however, not significantly affect my results (Kube, 2001). This situation is different if the errors in the geometry parameters are larger than only a few degrees.

\subsubsection{Observational data}

UZ For was observed with HST on June 11, 1992. A detailed description of the data is given by Stockman and Schmidt (1996). I summarize here only the relevant points.

Fast FOS/G160L spectroscopy with a time resolution of $1.6914 \mathrm{~s}$ was obtained, covering two entire eclipses in the phase interval $\phi=0.87 \ldots 1.07$. The two eclipses were observed starting at 05:05:33 UTC ('orbit 1') and 11:25:39 UTC ('orbit 2'). The spectra cover the range $1180 \ldots 2500 \AA$ with a FWHM resolution of $\approx 7 \AA$. The mid-exposure times of the individual spectra were converted into binary orbital phases using the ephemeris of Warren et al. (1995). The average trailed spectrum is shown in Fig. 4.7.

In order to obtain a light curve dominated by the accretion stream, I use the continuum subtracted C IV $\lambda 1550$ emission from the trailed spectrum. The resulting light curves are shown in Fig. 4.8 for both orbits separately. To reduce the noise to a bearable amount, the light curves were rebinned to $5.07 \mathrm{~s}$ resulting in a phase resolution of $\Delta \phi=6.7 \times 10^{-4}$.

Note that the CIV light curve may in principle be contaminated by emission from the heated side of the secondary star. HST/GHRS observations of AM Her, which resolve the broad component originating in the stream and the narrow component originating on the secondary, show that the contribution of the narrow component to the total flux of C IV is unlikely to be larger than $10 \ldots 15 \%$ (Gänsicke et al., 1998). Furthermore, during the phase interval covered in the HST observations of UZ For, the 


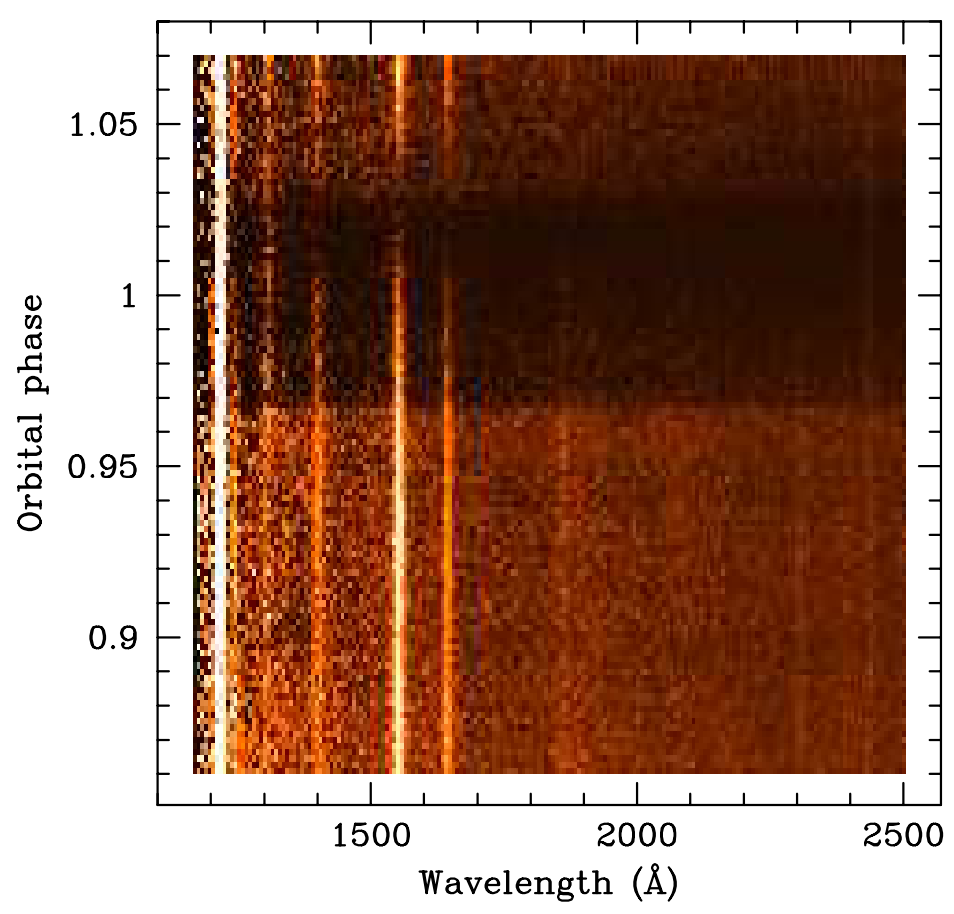

Figure 4.7: Trailed spectrum of UZ For, both observed orbits added and rebinned. The figure clearly shows the abrupt ingress and egress of the continuum source and the more gradual eclipse of the emission line source. It also shows a faint dip in the continuum and in the lines at $\phi=0.90$ which occurs when the magnetically funneled section of the accretion stream crosses the line of sight to the white dwarf.

irradiated hemisphere of the secondary is (almost) completely self-eclipsed, so that its $\mathrm{C}$ IV emission is minimized.

\subsubsection{Results}

The light curves show small, but significant differences for the two orbits (Fig. 4.8). In orbit 1 , the dip at $\phi=0.9$ is slightly deeper than in orbit 2 . However, this very small feature has only a marginal effect on the results. The dip is well known from X-Ray and EUV observations (Warren et al., 1995) and has been observed to move in phase between $\phi=0.88$ and 0.92 on timescales of months (Sirk and Howell, 1998). The ingress of the accretion stream into eclipse is much smoother in orbit 1 than in orbit 2 , where an intermediate brightness level around $\phi=0.98$ with a flatter slope is seen. The C IV intensity maps resulting from the fits are shown in Fig. 4.9 for each observation interval separately. In Fig. 4.10, I show the relative intensity distributions of 10 fit runs for each orbit, proving that the algorithm finds the same result (except for noise) for each run. In Fig. 4.9, the resulting map from one arbitrary fit is shown. 

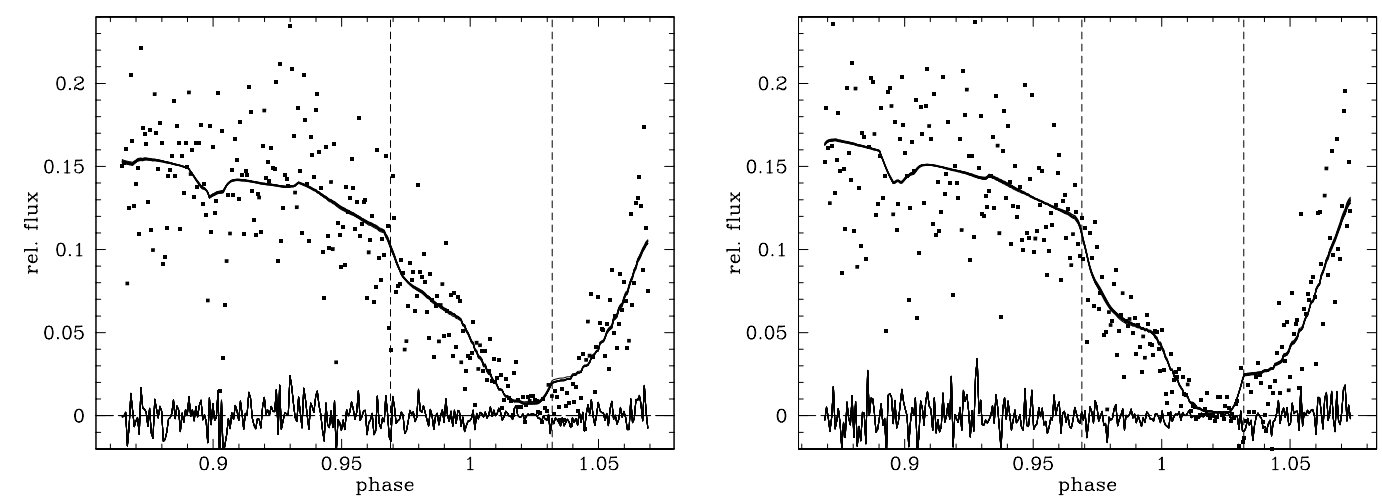

Figure 4.8: Extracted C IV light curves and best fits. Left: orbit 1, right: orbit 2. In each panel, ten light curves from different fit runs are overplotted (hard to recognize) to show the stability of the fit. The residuals are scaled down by a factor of 4 for clarity. The vertical dashed lines mark the ingress and egress of the white dwarf and, hence, approximately the begin of the ingress and egress of the magnetically funneled accretion stream.

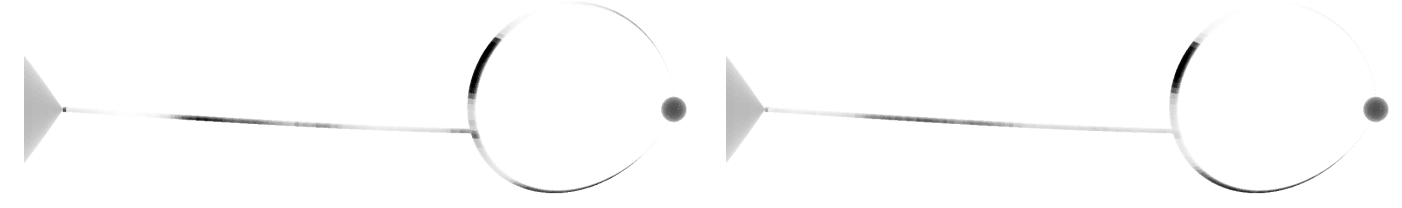

Figure 4.9: Resulting intensity maps of the accretion stream in UZ For. Left: Orbit 1, right: Orbit 2. Bright regions are printed in black, dark regions in white.
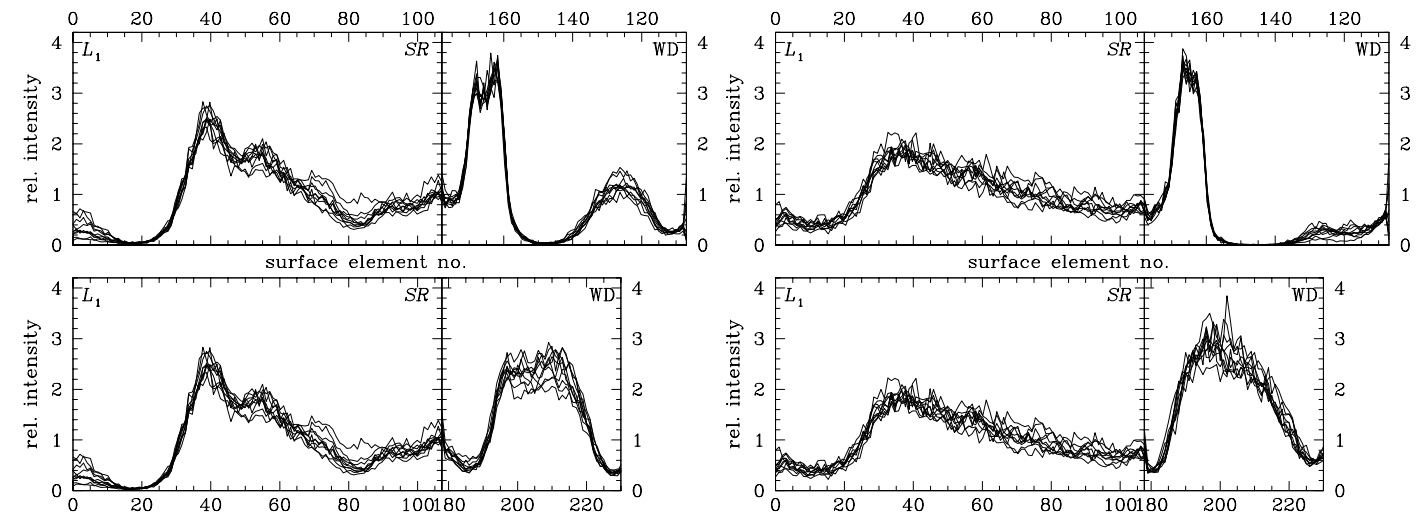

Figure 4.10: Resulting intensity distributions of the accretion stream in UZ For. Left: Orbit 1, right: Orbit 2. The results of 10 individual fit runs are overplotted in one graph to show the consistency of the fits. For an explanation of the plots see Fig. 4.4. 


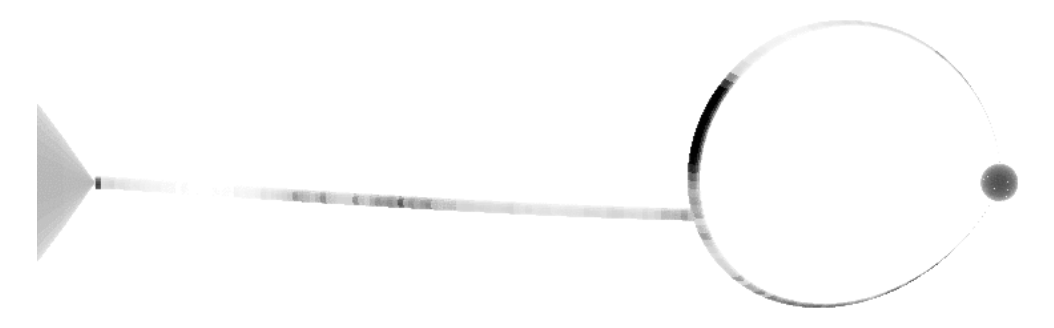

Figure 4.11: Intensity map of the accretion stream for orbit 1 . The light curve used to generate this map was changed from the observed so that for each phase point the flux was randomly modified with a gaussian with $\sigma$ as described in Sect. 4.4.2.

The brightness maps for the two orbits show common features and differences. Common in both reconstructed maps are the bright regions (1) on the ballistic stream, (2) on the dipole stream above the orbital plane, and (3) on the dipole stream below the orbital plane. In orbit 1 , there is an additional bright region on the northern dipole stream which appears as a mirror image of region 3, denomitad 3b. The difference between both maps is found in the presence/absence of region $3 \mathrm{~b}$, and in the different sharpness of region 1, which is much brighter and more peaked in orbit 1 than in orbit 2 .

Remarkable is that there is no bright region at the coupling region SR, where one would expect dissipative heating when the matter rams into the magnetic field and is decelerated. I will comment on this result in Sect. 4.5.4.

The sharp upper border of region 2 has to be discussed separately: As one can see from the data, the flux of the CIV $\lambda 1550$ emission ceases completely in the phase interval $\phi \approx 0.01 \ldots 0.03$. Hence, all parts of the accretion stream which are not eclipsed during this phase interval can not emit light in C IV $\lambda 1550$. For the assumed geometry of UZ For, parts of the northern dipole stream remain visible throughout the eclipse. Thus, the sharp limitation of region 2 marks the border between those surface elements which are always visible and those which dissappear behind the secondary star. Uncertainties in the geometry could affect the location of the northern boundary of region 2, but should not change the general result, namely that there is emission above the orbital plane that accounts for a large part of the total stream emission in C IV $\lambda 1550$.

Region 3b has to be understood as an artifact: During orbit 1, the observed flux level at maximum emission line eclipse $(\phi=0.01 \ldots 0.03)$ does not drop to zero. Hence, the algorithm places intensity on the surface elements of the accretion stream which are still visible at that phase. Apparently, the evolution strategy tends to place these residual emission not uniformly on all the visible surface elements but on those closer to the WD, which leads to an intensity pattern that resembles the more intense region on the southern side of the dipole stream.

To underpin the fact that regions 1, 2, and 3 in the map are real features and not just regions which result by random fluctuations in the data, I test what happens to the 
reconstruction if the input light curve is changend. For the calculation which results in the map shown in Fig. 4.11, I generated a modified light curve from the data for orbit 1. For each phase step, I modified the flux, so that $F^{\prime}(\phi)=F(\phi)+1 / 2 \cdot \sigma(\phi) \cdot G$ is the new value. $\sigma(\phi)$ is the local standard deviation as defined in Sect. 4.4.2, $G$ are gaussian-distributed random values. Since the map from the light curve $F^{\prime}(\phi)$ does not show significant differences from the map corresponding to the original data $F(\phi)$ (Fig. 4.9), I conclude that the features 1,2 , and 3 are real.

\subsubsection{Discussion}

I have, for the first time, mapped the accretion stream in a polar in the light of a high-excitation ultraviolet line with a complete $3 \mathrm{~d}$ model of an optically thick stream. I have found three different bright regions on the stream, but no strong emission at the stagnation point of the ballistic stream. In the following I will discuss the physical processes which may lead to an emission structure like the one observed.

\section{Emission of the ballistic stream}

As mentioned in Sect. 4.2, single-particle trajectories with different inital directions diverge after the injection at $L_{1}$, but converge again at a point approximately one third of the way between $L_{1}$ and the stagnation region. This is where one finds emission in the line of C IV $\lambda 1550$. Possibly the kinetic properties of the stream lead to a compression of the accreted matter, resulting in localized heating. After the convergence point, the single-particle trajectories diverge slowly and follow a nearly straight path without any further stricture. Hydrodynamical modeling of the ballistic part of the stream is required to substantiate this hypothesis.

\section{Absence of emission at the stagnation point}

In the classical model of polars, it is assumed that the ballistically infalling matter couples onto the magnetic field in the stagnation region with associated dissipation of kinetic energy (e.g. Hameury et al., 1986). Thus, one would expect a bright region near SR. The absence of C IV $\lambda 1550$ emision in the stagnation region could be due to the fact that there is no strong heating in the coupling region. Dissipation near SR can be avoided if the material is continously stripped from the ballistic stream and couples softly onto the field lines, as proposed by Heerlein et al. (1999) for HU Aquarii.

Another possibility is that the matter is decelerated near SR, resulting in an increase in the density. This may result in an increase of the continuum optical depth, and, therefore, in a decrease of the C IV $\lambda 1550$ equivalent width. 


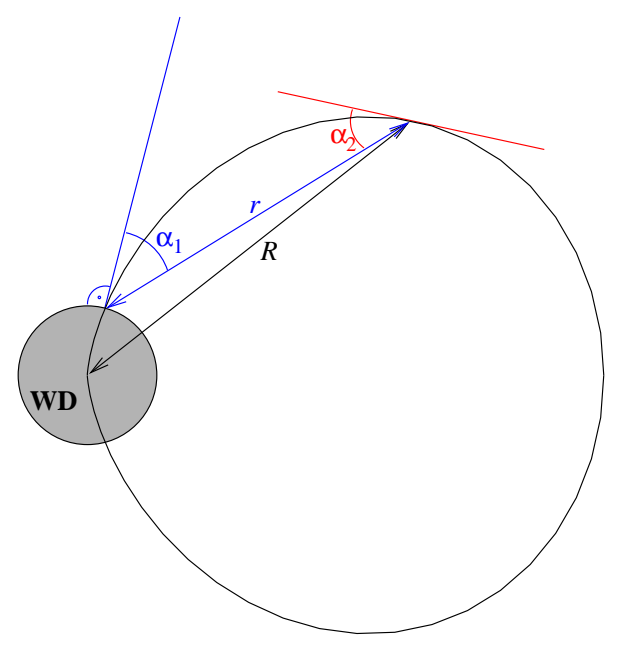

Figure 4.12: Dipole field line configuration. See Sect. 4.5.4.

\section{Emission of the dipole stream}

On the dipole section of the stream, there are two generally different emission regions: The bright and small region above the stagnation point and the broader regions near the accretion poles of the white dwarf.

Near the accretion spots: On the magnetically funneled stream, there is one region of line emission (3) which I assume to be due to photoionization by high-energy radiation from the accretion spot. The mirror region $3 \mathrm{~b}$ is an artifact which is created by the mapping algorithm to account for the non-zero flux level in orbit 1 in the phase interval $\phi=0.01 \ldots 0.03$ (see Sect. 4.5.3). Even though the distribution of C IV emission on the accretion stream does not reflect the irradiation pattern in a straigthforward way, the presence of CIV emission at a certain location of the stream requires that this point is irradiated, if there is no other mechanism creating C IV line emission. In Fig. 4.12, I define the angles $\alpha_{1}$ and $\alpha_{2}$ and the distances $r$ and $R$. Assuming a point-like emission region at the accretion spot, the absorbed energy flux per unit area caused by illumiation from the accretion regions varies as $\cos \alpha_{1} \sin \alpha_{2} R^{3 / 2} / r^{2}$. The structure of this equation shows that the illumination of the accretion stream is at maximum somewhere between the accretion region and the point with $\alpha_{1}=0$. This corresponds to the brightness distribution which I derive from the data.

I suggest the following interpretation of the emission region above the stagnation point: Independent of whether the C IV emission in this region is due to photoionozation or collisional excitation, a higher density than in other sections of the stream is required. Matter which couples in SR onto the field lines has enough kinetic energy to initially rise northward from the orbital plane against the gravitational potential of the white dwarf. If the kinetic energy is not sufficient to overcome the potential summit on the 
field line, the matter will stagnate and eventually fall back towards the orbital plane, where it collides with further material flowing up. This may lead to shock heating with subsequent emission of $\mathrm{C}$ IV $\lambda 1550$. Alternatively, the photoinization of the region of increased density may suffice to create the emission peak. Yet another possibility is that the matter is heated by cyclotron radiation from the accretion column which emerges preferentially in direction perpendicular to the field direction. Crude estimates show, however, that the energy of the cyclotron emission does not suffice to produce such a prominent feature as observed. A detailed understanding of the emission processes in the accretion stream involves a high level of magnetohydrodynamical simulations and radiation transfer calculations, which is, clearly, beyond the scope of this thesis. 


\section{Chapter 5}

\section{"Full-Orbit Eclipse Mapping"}

In this chapter, I describe the extension of the eclipse mapping method to full-orbit data. For convenience, I use the abbrevation FOEM for "Full-Orbit Eclipse Mapping".

\subsection{Method}

\subsubsection{Eclipse mapping outside of the eclipse}

The full-orbit eclipse mapping (FOEM) method is an extension of the classical eclipse mapping procedure. It is an attempt to reconstruct the spatial image by the means of light curve synthesis, which is - in contrast to the eclipse mapping method - done over the full orbit. While the eclipse mapping method as described in the previous chapter relies mainly on the ingress and egress of the occultation of the accretions stream (or disk) by the secondary star, the FOEM method uses the different aspects of the mapped object during the binary star orbit.

To allow that different viewing angles have an influence on the observed flux, one has to assume that the emission is optically thick. For emission lines in polars, this assumption is likely to be correct, as we can see for example from the observed emission line light curves. These light curves show double humps, which are in phase with the time when the observer sees the accretion stream at its largest projected area, i.e. around phases $\phi \approx 0.25$ and $\phi \approx 0.75$ (e.g. HU Aqr, Fig. 5.8). One could argue, that this variation comes from different projected areas of the secondary star (which would show the same phase behaviour). But from high-resolution spectroscopy, it is clear that most of the line emission comes from high velocity components which are identified with the stream. Also, the secondary star would be visible during the eclipse, while the emission line completely vanishes in that phase interval. However, there is still some contribution to the lines emission from the illuminated front side of the secondary star - this is the 
narrow line component visible in highly resolved spectra (Schwope et al., 1997a). This contribution has been removed from the data used in this chapter.

\subsubsection{2-dimensionality of the map}

FOEM allows the reconstruction of a $2 \mathrm{~d}$ texture on the $3 \mathrm{~d}$ geometry of a polar. This is different from the eclipse mapping (EM) method described in Chapt. 4, where I kept the intensity on the accretion stream the same for rings on the stream. That was neccessary, since only a small phase interval was used for EM. Since in FOEM every part of the accretion stream is seen from different viewing angles, it is reasonable to drop the restriction of having the same intensity of the stream in every direction perpendicular to the streaming direction. Hence, the resulting map is the $2 \mathrm{~d}$ surface of a $3 \mathrm{~d}$ object.

\subsubsection{Fitting algorithm}

The algorithm used to fit the intensities of the surface elements in such a way that the resulting light curve resembles the observed light curve, is done exactly as for the eclipse mapping application described in Sect. 4.3: An evolution strategy varies iteratively the intensities $I_{i}$ to find

$$
F_{\text {obs }}(\phi) \stackrel{!}{=} F_{\text {model }}(\phi)=\sum_{i \in \mathcal{V}(\phi)} I_{i} A_{i}(\phi) .
$$

$A_{i}$ holds the information about the geometry of the surface elements, which for the assumed optically thick behaviour is the projected area of the $i$-th surface. $\mathcal{V}(\phi)$ is the set of visible surface at phase $\phi$.

To account for the limited information content in the data, the entropy of the resulting map is maximized simultanously.

\subsection{4 "Information content"}

The amount of information for each surface element of the accretion stream depends on the accumulated change of the projected surface area of the element. If $A_{i}(\phi)$ is the projected surface area of the $i$-th surface element at phase $\phi$ (observed phases are $\phi_{1}$ to $\phi_{n}$, one can define the total change of the projected surface area over the orbit as

$$
\Delta A_{i}=\sum_{j=2}^{n}\left|A_{i}\left(\phi_{j-1}\right)-A_{i}\left(\phi_{j}\right)\right|+\left|A_{i}\left(\phi_{n}\right)-A_{i}\left(\phi_{1}\right)\right|
$$

A surface with a high $\Delta A_{i}$ shows many changes of its projected surface. Normalizing $\Delta A_{i}$ by dividing by the real surface area $A_{i}$ gives a measure of the relative amount 


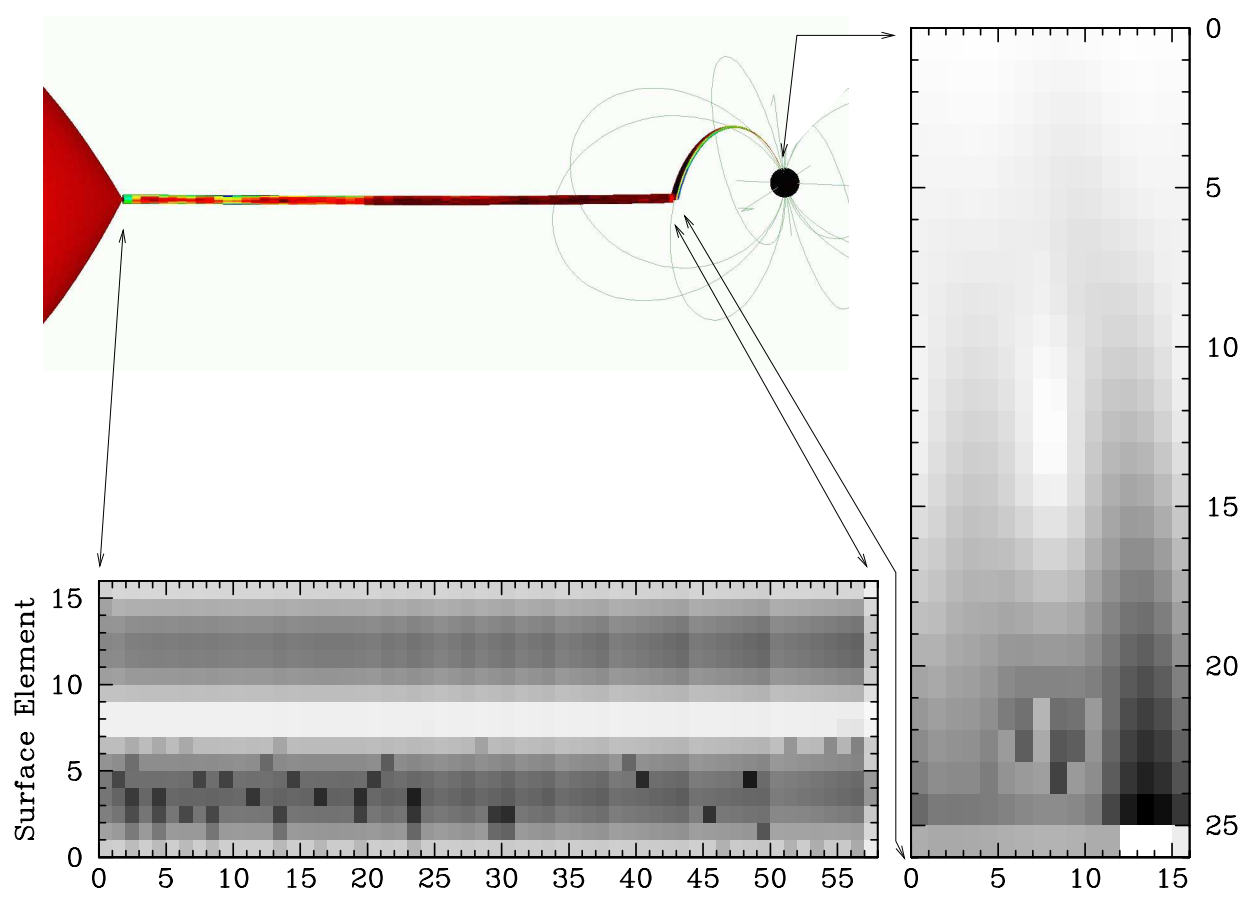

Figure 5.1: Information content for the different surface elements in HU Aqr. The grayscale image shows the value $\frac{\Delta A_{i}}{A_{i}}$ as described in Eq. (5.2). Dark surface elements show a large variation of the apparent size during one orbit, bright elements are either visible at all or at no times. The pixels translate into the $3 \mathrm{~d}$ geometry as indicated by the arrows. The colors on the $3 \mathrm{~d}$ images are as in the results (Fig. 5.9). Elements on the ballistic stream with $y \in[1,8]$ are on the "inside", i.e., they are seen from the WD. Those on the magnetic stream are numbered that the "inside elements" are around $x=8$. The apparently random speckles on the ballistic stream (lower-left panel, pixels $y \in[1,8]$ ) are an artifact from the eclipse of parts of the ballistic stream by the dipole stream combined with a coarse phase resolution.

of information which is contained in the data set $F\left(\phi_{0}, \ldots, \phi_{n}\right)$. In Fig. 5.1 the "information content" for the accretion stream surfaces of HU Aqr is shown. Note that this analysis only gives a qualitative view on the reliability of the features at different positions of the map.

\subsection{Test calculations}

As in the last chapter on eclipse mapping, I show tests of the quality of FOEM reconstructions. These tests allow to gain insight into typical artifacts of the method as well as in the reliability of reconstructions under controlled circumstances. A wide set of artifical noise can be tested to experiment with the range of the input data quality. 
Table 5.1: System parameters for the FOEM test runs.

\begin{tabular}{lc}
\hline \hline \multicolumn{1}{c}{ System parameter } & HU Aqr \\
\hline mass ratio $Q=M_{1} / M_{2}$ & 4 \\
total mass $M=M_{1}+M_{2}$ & $1.1 M_{\odot}$ \\
orbital period $P$ & $125.021 \mathrm{~min}$ \\
orbital separation $a$ & $5.93 \times 10^{10} \mathrm{~cm}$ \\
inclination $i$ & $85.6^{\circ}$ \\
radius of WD $R_{1}$ & $6.0 \times 10^{8} \mathrm{~cm}$ \\
\hline dipole tilt $\beta$ & $14^{\circ}$ \\
dipole azimuth $\Psi$ & $223^{\circ}$ \\
azimuth of $S \Psi_{S}$ & $43^{\circ}$ \\
\hline \hline
\end{tabular}

\subsubsection{Setup}

As a test of the FOEM method, I set up three test scenarios. These scenarios resemble three real polars, namely AM Her, UZ For, and HU Aqr. The difference between these systems is: UZ For and HU Aqr are eclipsing systems, while AM Her is not; UZ For accretes on both magnetic poles, HU Aqr and AM Her only on one pole.

Three different purely artificial intensity maps were used, from which I calculated the synthetic light curves: (mp1) a bright spot on the dipole stream at the point closest to the stagnation region, facing the white dwarf (3 of 16 surface elements in azimuth around the stream), contrast 1:50, (mp2) the same, but contrast only 1:10, (mz) a zebra like pattern on the ballistic stream, three bright strips with a contrast of $1: 10$. The light curves were calculated for (p1) 200 phase steps over the full orbit, (p2) 20 phase steps over the full orbit, (p3) 50 phase steps around the eclipse, (p4) 200 phase steps around the eclipse (p3 and p4 only for UZ For and HU Aqr). As a last parameter, I tried to fit not only systems with the correct (g0) geometry, but also with voluntarily slightly incorrect system geometries (g1): $i$ was wrong by $15^{\circ}$ for AM Her and $3^{\circ}$ for HU Aqr and UZ For. The mass ratio for the latter systems has been adjusted to keep the duration of the eclipse correct. Additionally, all the angles describing the magnetic configuration have been changed by $10^{\circ}$ each, again correcting $\Psi_{S}$ to the correct position of the "dip" in the eclipsing systems. To the synthetic light curves, I added Gaussian noise with an constant (bias) and a contribution proportional to the intensity. The S/N was $\infty(\mathrm{r} 0), \approx 25(\mathrm{r} 1)$, or $\approx 4(\mathrm{r} 2)$. From that grid of 240 test calculations, I show the most important results for HU Aqr, only. The results of the test calculations for the UZ For and AM Her geometry show the same behaviour. The system parameters for the HU Aqr geometry are listed in Tab. 5.1. 


\subsubsection{Results for the HU Aqr geometry}

The results of the test calculations are shown in the Figs. 5.2 to 5.7. The different panels of the figures contain the following information. Top left: the "observed" (i.e. synthetic and noisified) light curve with its statistical errors as dots with error bars. Therein as a line, hard to recognize in most cases, the light curve which results from the best fitting intensity map. For clarity, the residuals (observed - calculated) are shown in units of the statistical error in the lower part of the top left panel. Bottom left and bottom right: This is the negative grayscale map of the surface intensities on the accretion stream. The ballistic stream runs from left to right in the bottom left panel, the numbers indicating the indizies of the surface elements. The $y$-axis on this panel goes with the azimuth around the stream. Elements with $y=8$ hold the surfaces which are on top of the ballistic stream elements with $y=4$ are those on the trailing side in orbital motion (seen at phase $\phi \approx 0.25$ ). The bottom right panel shows the surface elements on the magnetically dominated stream. Here of cause the $x$-value denominates the azimuth around the stream with $x=8$ indicating the surface elements facing the white dwarf or the "inside" of the dipole loop. The surface elements at the bottom are those closest to the white dwarf. The coupling region is at surface elements with $y=50$ (see also Fig. 5.1). Please note, that the $y$-direction of the dipole map is changed from these synthetic maps with respect to the real HU Aqr maps later in this chapter. The top right panel shows the logarithmic intensity histogram of the map, not corrected for the surface element size (which is not constant).

The Figs. 5.2, 5.3, and 5.4 show the reconstructions of the intensity maps for the "correct" geometry with $\mathrm{S} / \mathrm{N} \approx 10$ with an emission "point" with contrast 1:50 and 1:10 and the "zebra" again with 1:10 contrast.

The first striking result is the very well defined reconstruction of the 1:50 point feature (Fig. 5.2). The small difference between the input image and its reconstruction is found in a small displacement of the bright spot of $\approx 1$ surface element in azimuth and some additional brightness on the upper and lower parts of the first half of the ballistic stream.

The reconstruction of the 1:10 point (Fig. 5.3) is less favourable. The bright spot on the dipole stream has split up into two spots, and there is some additional brightness close to the $x=10$ surface elements on the ballistic stream.

The 1:10 zebra map can hardly be recognized in its reconstruction. From the three zebra stripes, only the first is visible on both sides of the ballistic stream. The second stripe is only visible on the leading side, the third stripe only on the trailing side of the stream with respect to the orbital motion. The only hint in the data of the zebra-like character of the input map can be seen as a step in the eclipse ingress at phase 0.95, so the result is not surprising.

For the next three tests, we only look at the 1:50 point. 


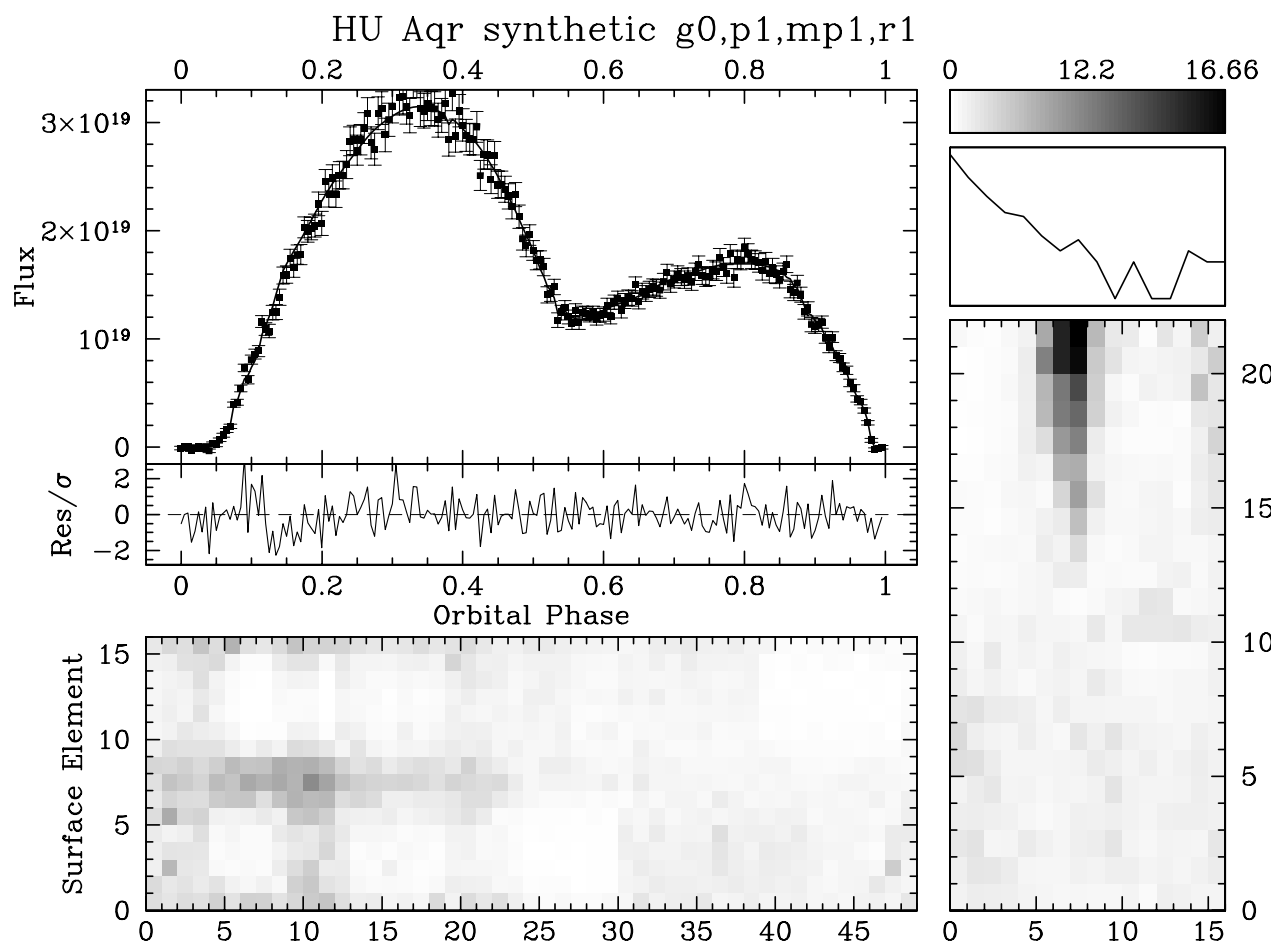

Figure 5.2: Synthetic light curve and FOEM reconstruction.

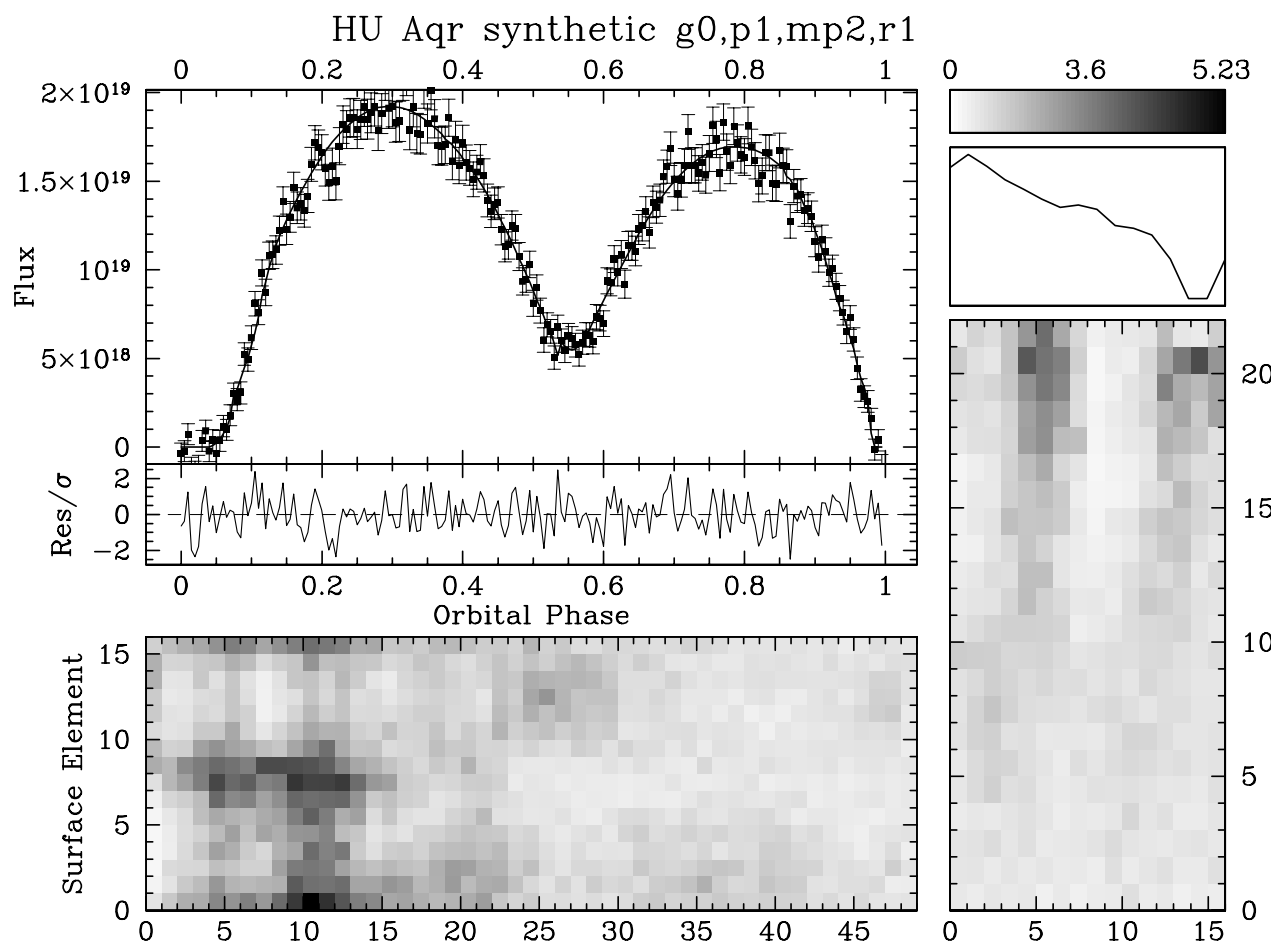

Figure 5.3: Synthetic light curve and FOEM reconstruction. 


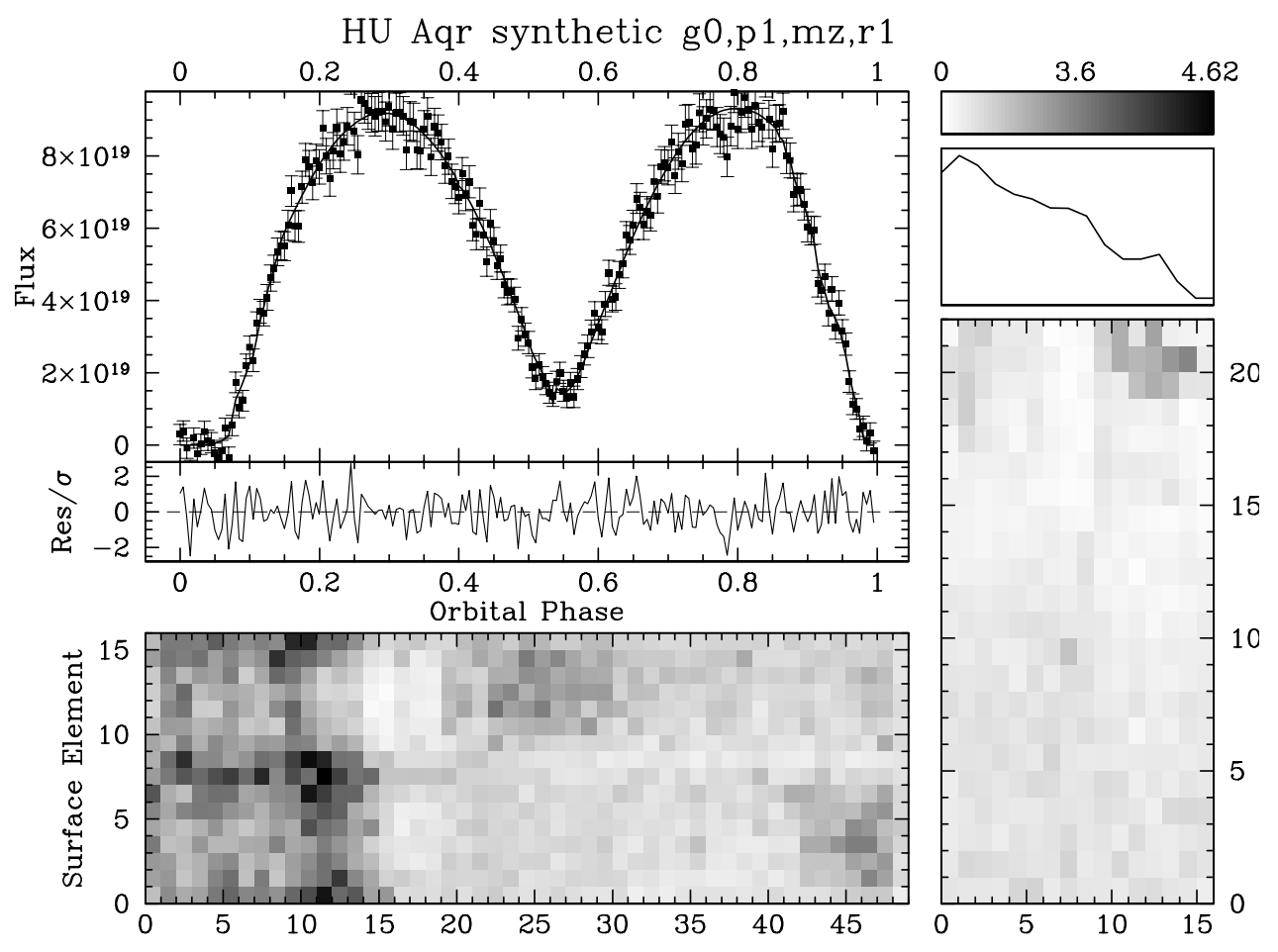

Figure 5.4: Synthetic light curve and FOEM reconstruction.

The next figure, 5.5, shows the results for the "wrong" geometry. The map is very similar to the one with the "correct" geometry in Fig. 5.2. The displacement of the spot from the position at $x=8$ on the dipole stream is still a bit more pronounced, yet the reconstruction shows no additional artifacts or errors.

The reconstruction attempt with only data around the eclipse (Fig. 5.6) had to fail. No information about the bright spot whatsoever is visible during the phase interval of $\phi=0.8 \ldots 1.2$. So the absence of prominent features is expected. Anyway, there are some low-contrast features, especially on the first third of the ballistic stream, which seem to be intrinsic to the mapping method.

Even the reconstruction of data with a $\mathrm{S} / \mathrm{N}$ of $\approx 4$ leads to satisfying results: In Fig. 5.7 the spot on the dipole stream is visible. Similar to the 1:10 spot discussed earlier, it is now split in two different spots, with the fragment close to the original position being much stronger than the artifact. On the ballistic stream, some additional brightness in the first quarter is seen. 

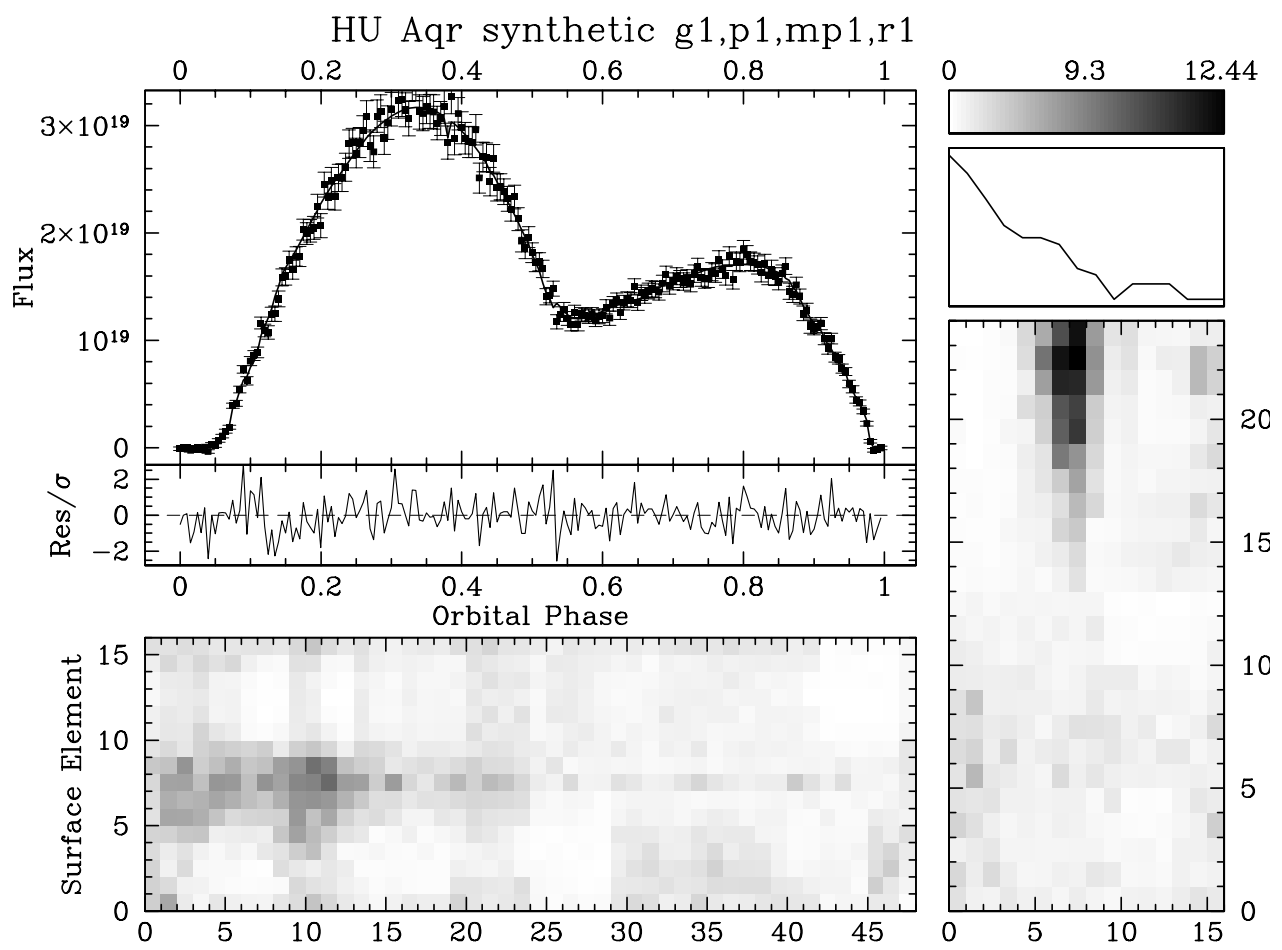

Figure 5.5: Synthetic light curve and FOEM reconstruction. In this case, FOEM was reduced to data around the eclipse, so strictly speaking this is eclipse mapping with too many degrees of freedom.

\subsection{Full-orbit eclipse mapping of HU Aqr}

HU Aqr was observed on 1993 August 17-18 with the TWIN spectrograph at the $3.5 \mathrm{~m}$ telescope on the Calar Alto observatory (Schwope et al., 1997a). For the following analysis, I use the emission line light curves of He II $\lambda 4686$ and $\mathrm{H} \beta$, which have been reduced by the emission originating from the illuminated front side of the secondary star. The data, which is shown with errorbars in Fig. 5.8, large panel at top left each, is the same as that analyzed by Vrielmann and Schwope (2001), hereafter called VS01. The geometry of HU Aqr used for the FOEM-calculations is the same as described in Tab. 5.1 and is identical to the parameters used in VS01.

The light curves show a pronounced double-hump structure which in general is a sign for a bright accretion stream. The maxima are at $\phi=0.24$ and $\phi=0.75$, which is the phasing that would be expected by the projection effects of an emitting ballistic accretion stream. Additionally, the eclipse produced by the secondary star is seen in the interval $\phi=0.97 \ldots 1.05$. In the light of He II $\lambda 4686$, the contrast between the two humps is not as pronounced as in the light of $\mathrm{H} \beta$. The phasing of the maxima is the same in both emission lines. 


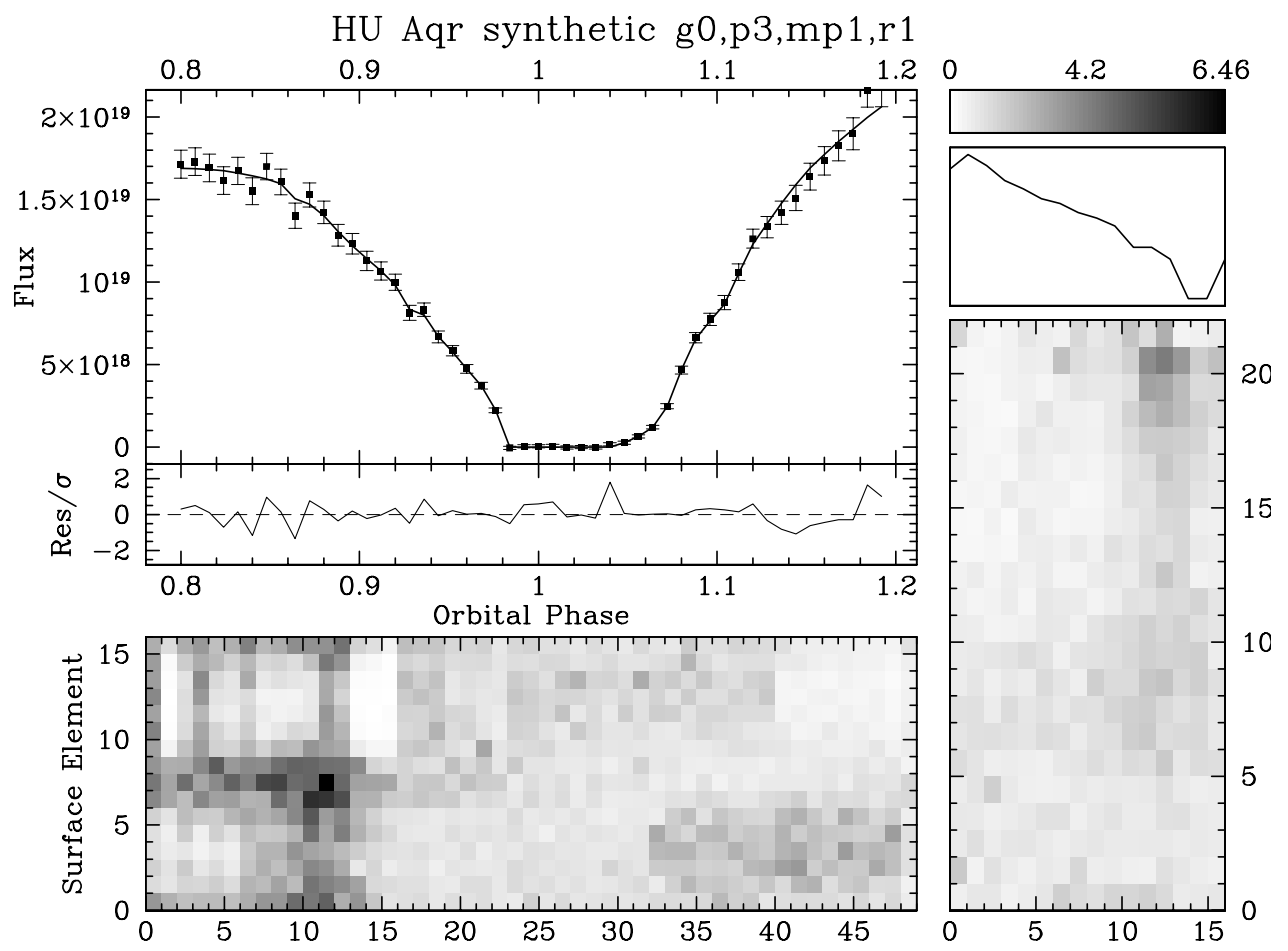

Figure 5.6: Synthetic light curve and FOEM reconstruction.

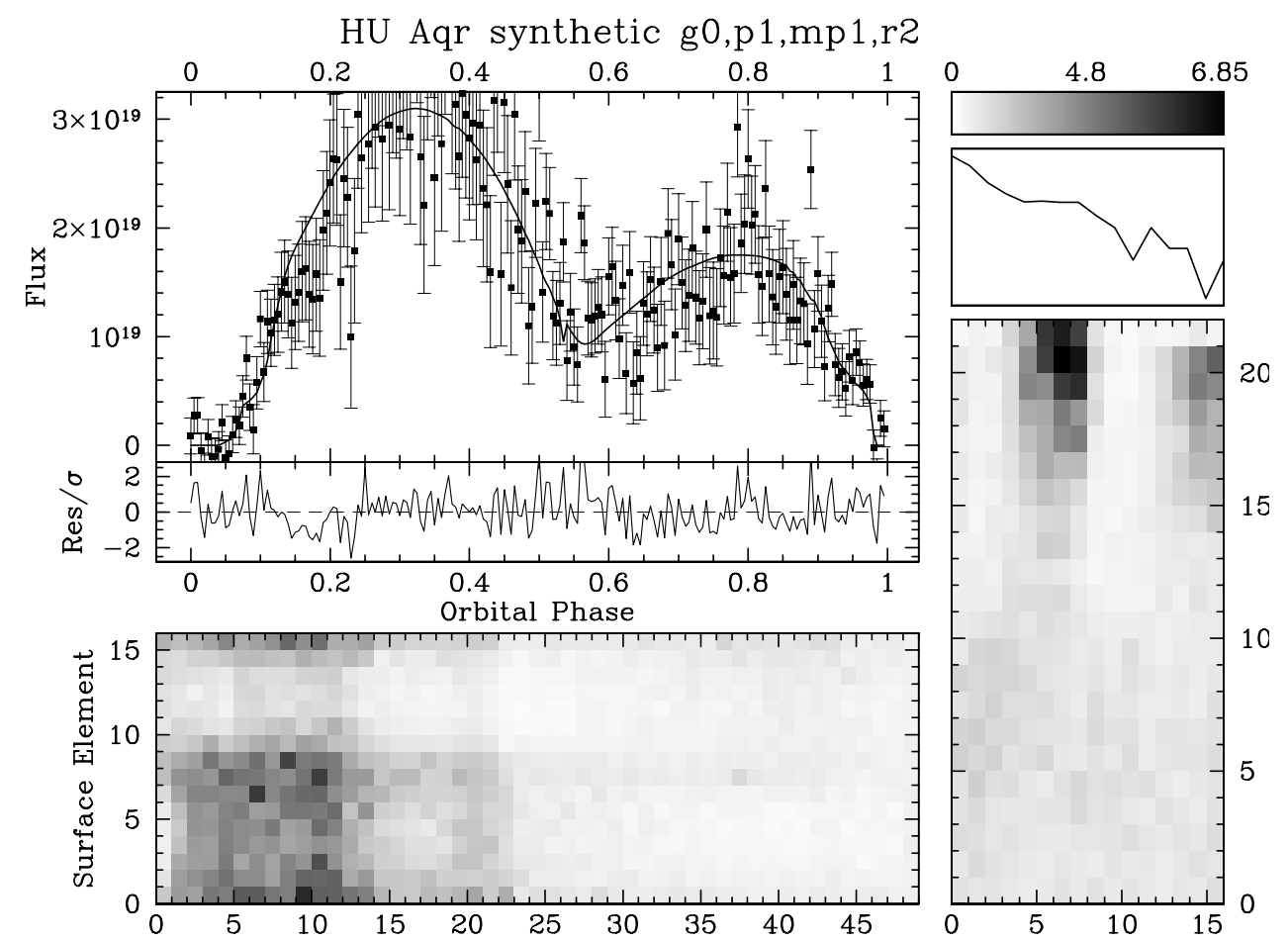

Figure 5.7: Synthetic light curve and FOEM reconstruction. 

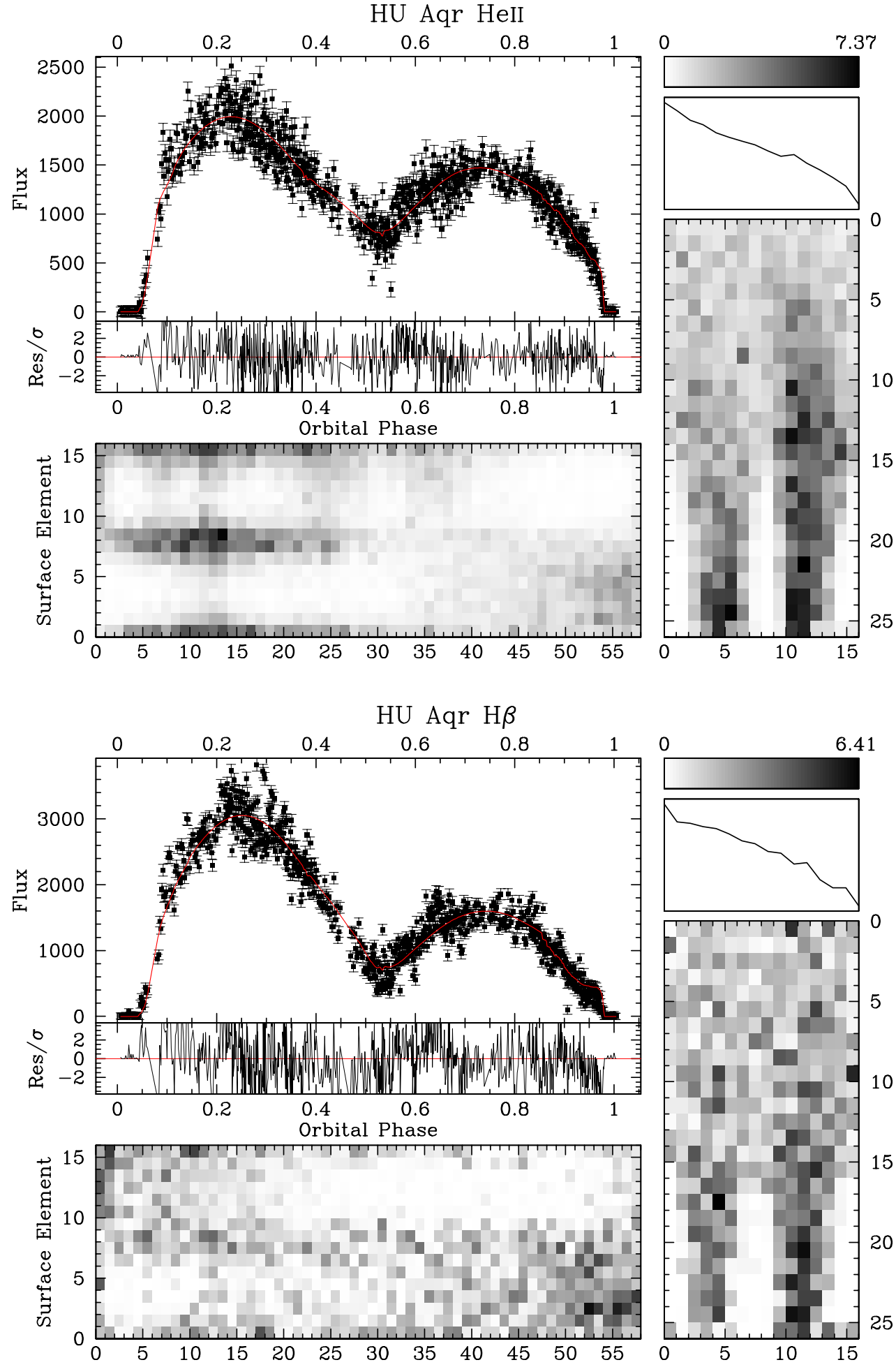

Figure 5.8: HU Aqr emission line light curves, reconstructions, and the derived intensity maps. Top: He II $\lambda 4686$, bottom: $\mathrm{H} \beta$. 
In my reconstructions of the accretion stream brightness, there are three sources of emission which can be trusted. The structure in the first 25 surface elements of the ballistic stream (lower panel), where emission is located at the elements numbered 1 , 16 and 8, 9, does not belong to them. These surfaces are located on the top and the lower parts of the accretion stream and are known to show heavy artifacts from the tests shown before.

The main emission sources are

1. the last part of the ballistic stream on the side towards the white dwarf. These are the surface elements around $x=55, y=5$,

2. the magnetically funneled stream at the elements $x=5$ and $x=12$ along half of the dipole stream. These are the surface elements whose normal vector is perpendicular to the direction towards the white dwarf. In other words, the surfaces facing the white dwarf are not as bright as those from which the normal vector is perpendicular towards the direction to the white dwarf.

3. the part of the ballistic stream which is on the first third of the way towards the stagnation region. While not clearly visible in Fig. 5.8, it is readily detected in Fig. 5.9.

For a better understanding of the 3d-location and orientation of the resulting emission map, Fig. 5.9 shows 9 images of the accretion stream in the light of He II $\lambda 4686$ at the phases $\phi=0.1 \ldots 0.9$ in steps of $\Delta \phi=0.1$.

The first of the emission sources is also found by VS01. The second source, which is the part of the dipole stream which may only be illuminated grazingly by the white dwarf, is not claimed by VS01. However, if one looks at their resulting maps (Fig. 5 in their article), the interpretation as a bright region on the dipole stream which faces the white dwarf seems a bit fuzzy. Especially in He II $\lambda 4686$ their map also shows a two-fold emissivity at a position which has to be understood as described in my results above. Unfortunately, the grid for the accretion stream used by VS01 has only 12 segments around the stream, which seems too few for resolving a structure like the one detected by my FOEM application.

The third emission source, which is clearly visible in Fig. 5.9, is at least partially found by VS01, but considered as a numerical artifact. My interpretation is that that bright region on the first third of the ballistic stream is real. It accounts for the intensity increase in the phase interval $\phi \approx 0.095 \ldots 0.135$ and the decrease just before the eclipse at $\phi \approx 0.90 \ldots 0.95$. In these intervals, projection effects of an uniformly bright or dark stream only could not explain the observed light curves.

Looking at the location of the bright regions and the positions over an orbit allows the analysis of their plausibility: The main maximum at $\phi=0.24$ is a result of projection 


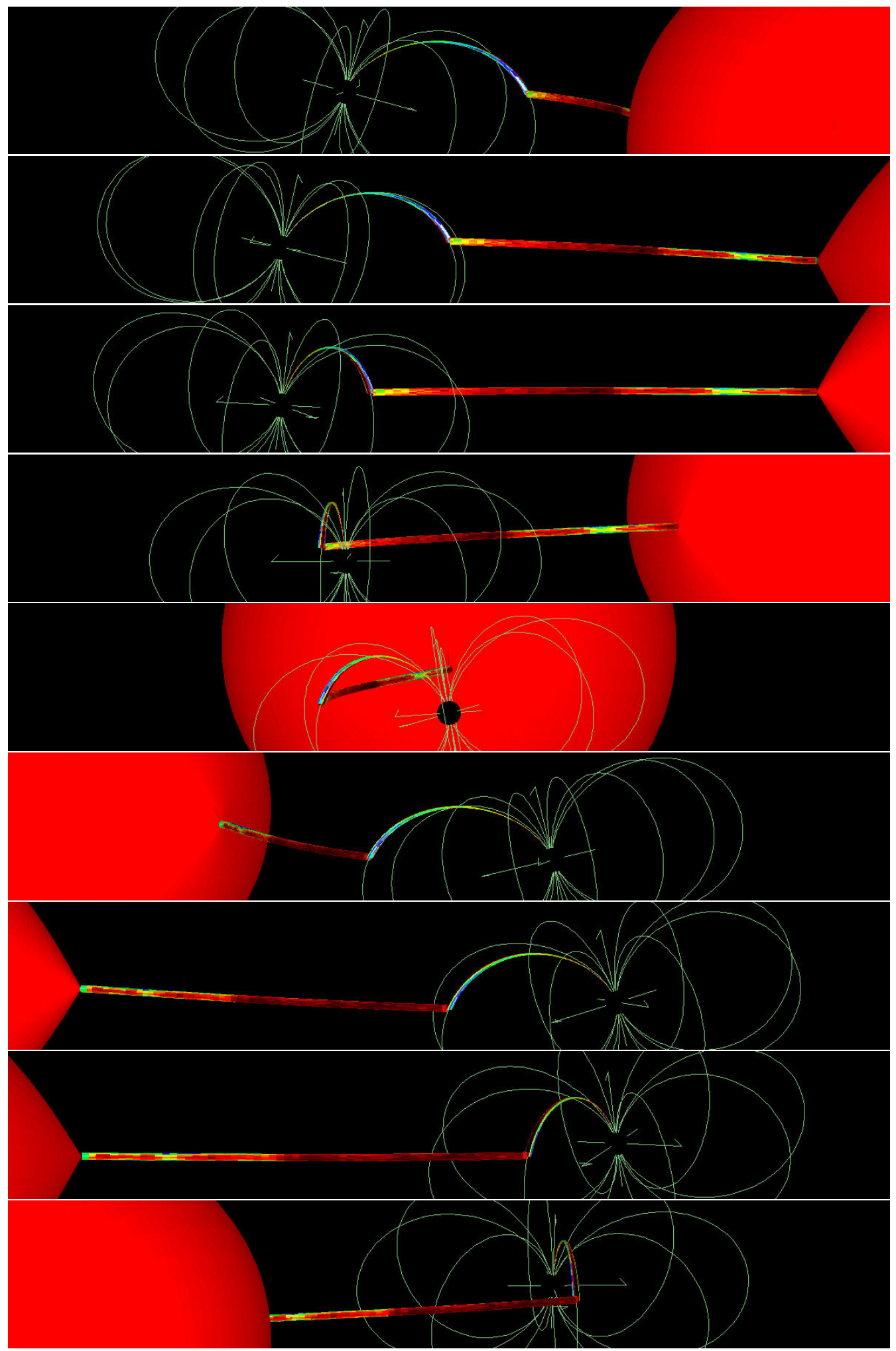

Figure 5.9: $3 \mathrm{~d}$ sequence of the accretion stream in HU Aqr in the light of He II $\lambda$ 4686. From top to bottom: $\phi=0.1 \ldots 0.9$ in steps of $\Delta \phi=0.1$. Figures created with my visualisation tool OGL-Star. The brightness on the stream is coded in the colors black-red-yellow-greencyan-blue-white. Additionally, the magnetic configuration of the WD is indicated by the thin green lines. 


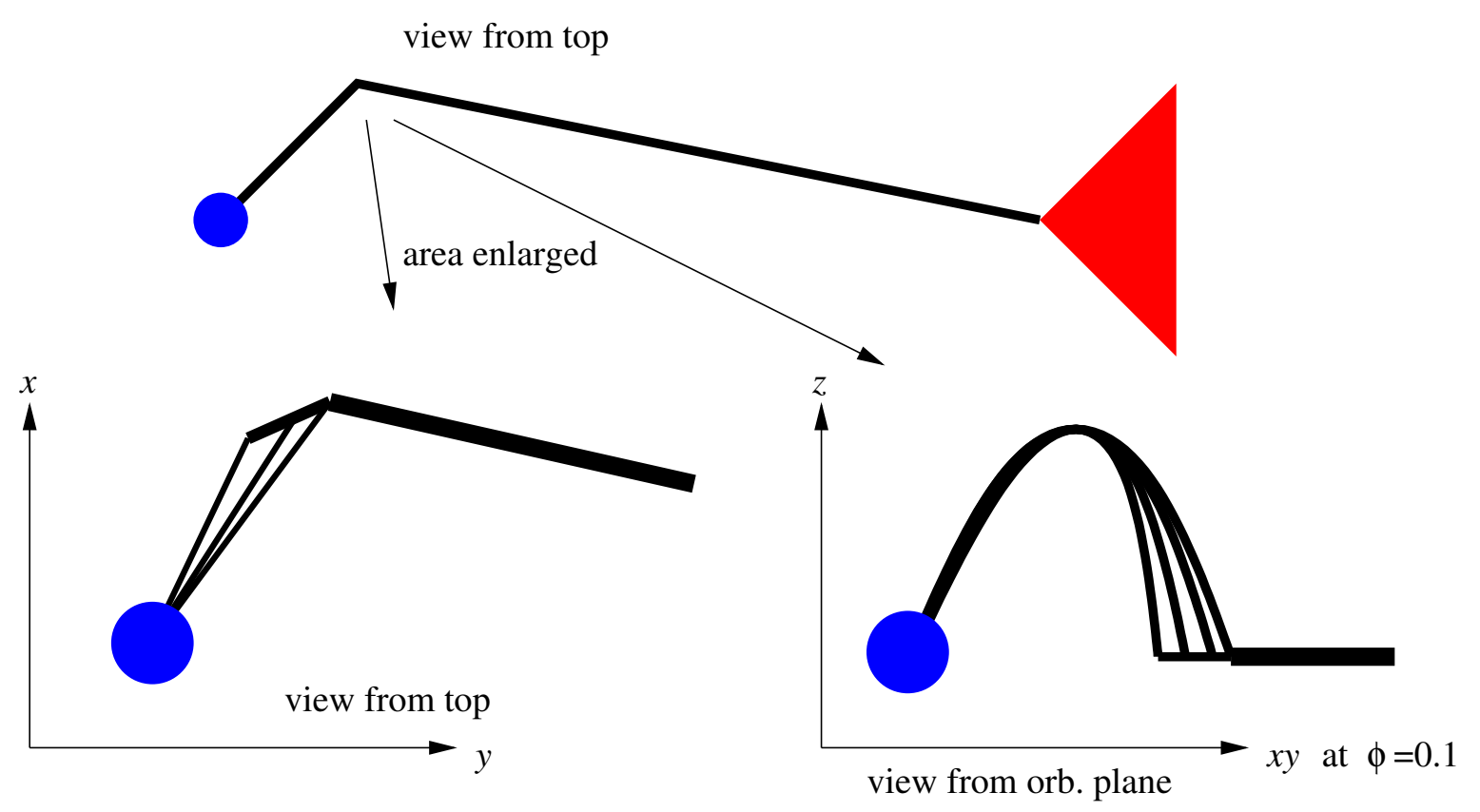

Figure 5.10: A model for a possible accretion curtain in HU Aqr. The top and the bottom left figure are as seen from on top of the orbital plane, while the bottom right panel shows the accretion curtain from the orbital plane at a point where the observer would be at $\phi \approx 0.1$. Compare to Fig. 5.9, top panel.

effects of the two bright regions on the ballistic stream, which are located on the first third and near the stagnation region. The secondary maximum at $\phi=0.75$ has its source mainly in the projection effects of the first thirds of the ballistic stream. The "outside" of the stagnation region is significantly dimmer than the inside. The bright borders of the dipole stream have their maximal contributions at $\phi \approx 0.1$ and $\phi \approx 0.6$ where they add to the projection induced light curve of the ballistic stream.

The fact, that the ballistic stream dominates the line emission underpinned by Doppler maps of HU Aqr, where emission is only detected at velocity locations compatible with the ballistic stream (Schwope et al., 1997a).

In the interpretation of the FOEM maps, one has to be careful. Not all of the assumptions made for the modeling of the light curve must be taken literally. The stream is modeled to have a constant cross section in the ballistic part and a cross section with a size coupled to the magnetic field in the dipole part. A real stream may have a different behaviour. Especially the bright regions of the dipole part could be interpreted not as "bright regions with constant size" but alternatively as "constant brightness regions with a different size" or a blend of both. Hence, the magnetically funneled stream may be extended in the direction towards the white dwarf, or - to be more general - in a direction which is seen with the maximum projected area at phases $\phi=0.1$ and $\phi=0.6$. In Fig. 5.10, a possible geometrical interpretation is sketeched: The infalling material 
is decelerated at the coupling region. That forces the trajectory to change towards the white dwarf. It couples onto the magnetic field, producing an accretion curtain, which extends not along the former ballistic field line, but along a line which is seen laterally at $\phi \approx 0.15$. The dipole stream would extend into a curtain. The resulting light curve would be undistinguishable from a circular stream which is bright at the places where the curtain would be extended.

The FOEM-method has shown that it can be used to create light curves that fit to the observed light curve very well. From the maps used to construct such a light curve, information about the emission structure on the accretion streams can be gained. In the example of HU Aqr, I was able to validate some of the results from Vrielmann and Schwope (2001), while adding new aspects to the interpretation of the emission from the magnetically funneled stream. 


\section{Chapter 6}

\section{Orbital Mapping}

In this chapter, I describe the core work of this thesis, the new method called "orbital mapping" (OMA). Since it is a complementary method to the well-known method of "Doppler mapping", I refer to the earlier chapter on that method (Sect. 3.4). I'll give a description of how the orbital mapping method works. With simulated data I perform tests of the orbital mapping method. Then I describe the new cataclysmic variable system, HS0455+83, in which I try to confirm the stream overflow model by using the orbital mapping method. I apply the OMA method to a second system, the SU UMa star V436 Cen, for which I derive the radial intensity profile of the accretion disk in two Balmer emission lines.

\subsection{Orbital mapping: a combination of eclipse- and Doppler mapping}

In contrast to Doppler mapping, which works mainly as a back projection of the observed data, the approach of orbital mapping is a forward synthesis of time resolved spectra. The two-dimensional data set, i.e. the flux at a given wavelength vs. time, can be written as

$$
F(\lambda, \phi)=\sum_{i=1}^{N} I_{i} C_{i}(\lambda, \phi) \quad \text { compare to Eq. (4.4) }
$$

if the surface is modeled as $N$ surface elements $i$, which have a contribution $C_{i}(\lambda, \phi)$ at a specific wavelength and orbital phase and an intensity of $I_{i}$ which is wavelength- and phase independent. Aim of the mapping process is to derive all intensities, hence to find the intensity vector $I_{i}$ for which Eq. (6.1) reproduces the observed time-resolved spectrum $F_{\text {obs }}(\lambda, \phi)$ as good as possible while the entropy of the resulting intensity map is at maximum. 
The tensor $C_{i}(\lambda, \phi)$ contains the information about the physical model of a CV as well as the information about the instrumental resolution and the radiation processes. The details of the generation of $C_{i}$ is described in the following section.

For convenience, the wavelength position $\lambda$ is converted to velocity coordinates using the Doppler shift for low velocities $(v \ll c)$ :

$$
v=c \cdot \frac{\lambda-\lambda_{0}}{\lambda_{0}}
$$

with $\lambda_{0}$ the rest wavelength of the observed spectral line. This allows to keep the $C_{i}(v, \phi)$ calculation the same no matter which line is used - as long as the physical assumptions remain the same, e.g. for the same emitting species as in the Balmer series.

\subsubsection{Emission line synthesis}

$C_{i}$ is calculated in two stages. In the first stage, the effective surface area of each element is calculated. If $A_{i}$ is the total surface area of the $i$-th surface element, the effective area at phase $\phi$ is either

$$
\begin{aligned}
& A_{i}(\phi)=A_{i} \cos \alpha \quad \text { (optically thick), or } \\
& A_{i}(\phi)=A_{i} \quad \text { (optically thin), or } \\
& A_{i}(\phi)=0 \quad \text { if the surface is eclipsed or } \cos \alpha<0
\end{aligned}
$$

with $\alpha$ the angle between the surface normal of the $i$-th surface and the direction towards the observer.

Now the line profile of the emission from the surface element has to be taken into account. There are two different mechanisms which I include in the single surface element line profile, (a) the intrinsic line width at the point of emission, and (b) the instrumental resolution. For both mechanisms, a Gaussian line profile is assumed. If the width for (a) is given by $\sigma_{\text {emiss }}$ and for (b) by $\sigma_{\text {instr }}$, the width of the resulting Gaussian is

$$
\sigma=\sqrt{\sigma_{\text {emiss }}^{2}+\sigma_{\text {instr }}^{2}}
$$

For the test calculations in Sect. 6.2, I use $\sigma_{\text {emiss }} \ll \sigma_{\text {instr }}$, hence $\sigma \approx \sigma_{\text {instr }}$.

The maximum of the Gaussian of a single surface element is determined by its apparent velocity at the observed phase. With $\vec{v}_{j}=\left(v_{x}, v_{y}, v_{z}\right)_{j}$ the velocity in the fixed frame at $\phi=0$ (see Fig. 3.3, p. 20), the observed velocity of element $j$ at phase $\phi$ is

$$
v_{j}(\phi)=\underbrace{\cos i \cdot v_{z, j}+\sin i}_{\text {inclination }} \underbrace{\left(v_{y, j} \sin 2 \pi \phi-v_{x, j} \cos 2 \pi \phi\right)}_{\text {phase }}
$$

In that equation, $i$ is the system inclination. 
The value for a $v$-continuous $C_{i}(v, \phi)$ would be

$$
C_{i}(v, \phi)=\frac{A_{i}}{\sigma \sqrt{2 \pi}} \exp \left(-\frac{\left(v-v_{i}(\phi)\right)^{2}}{2 \sigma^{2}}\right)
$$

Since $C_{i}$ is discretized into finite velocity intervals

$$
v_{j}=[\underbrace{v_{j, \mathrm{cent}}-\Delta v_{j} / 2}_{v_{j, 1}}, \underbrace{v_{j, \text { cent }}+\Delta v_{j} / 2}_{v_{j, 2}}]
$$

one has to integrate Eq. (6.6) to obtain

$$
C_{i, j, \phi}=\int_{v_{j, 1}}^{v_{j, 2}} C_{i}\left(v_{j}, \phi\right) \mathrm{d} v_{j}=\frac{A_{i}}{2}\left[\operatorname{erf}\left(\frac{v_{j, 2}-v_{i}(\phi)}{\sqrt{2} \sigma}\right)-\operatorname{erf}\left(\frac{v_{j, 1}-v_{i}(\phi)}{\sqrt{2} \sigma}\right)\right]
$$

with

$$
\operatorname{erf}(x):=\frac{2}{\sqrt{\pi}} \int_{0}^{x} \exp \left(-t^{2}\right) \mathrm{d} t
$$

as implemented in the standard $\mathrm{C}$ math library. $\Delta v_{j}$ is the size of the $j$-th velocity interval of the data set, while $v_{j \text {,cent }}$ is the center of the $j$-th velocity interval.

\subsubsection{Map generation}

The map which is to be reconstructed consists of (a) the geometrical information of the specific object, which is the $3 \mathrm{~d}$ grid model of the components (for the white dwarf, the accretion stream, and the secondary star see Sect. 4.2) and (b) the vector $\vec{I}$ holding the intensity information for all the $N$ surface elements. A bit sloppily, the vector itself may also be referred to as "map".

The map generation process is - as in the previous chapters - an iterative evolution strategy which tries to fit $\vec{I}$ to the observed data $F_{\text {obs }}(v, \phi)$ aiming to fulfill Eq. (6.1) with the additional condition that the map is as smooth as possible. In Sects. 4.3.2 and 4.3 .3 (pp. 30/31), the fitting method is described in more detail.

\subsubsection{The geometry of a disk system}

Since I had no suitable data available for the orbital mapping process in a polar (the class of systems used in the chapters before), I had to incorporate accretion disk models into my code. Nevertheless, the code's capabilities would also allow mapping in polars. 
Table 6.1: System parameters of the synthetic object CV Toy

\begin{aligned} & \hline \hline$M_{1} / M_{2}=1.4 \\ & M_{\text {tot }}=0.96 M_{\odot} \\ & P_{\text {orb }}=240 \mathrm{~min} \\ & i=75^{\circ} \\ & R_{\mathrm{WD}}=8.5 \times 10^{8} \mathrm{~cm} \\ & r_{\text {disk,in }}=R_{\mathrm{WD}} \\ & r_{\text {disk,out }}=0.30 a \\ & h_{1}=0.01 a \\ & h_{2}=1.3 \\ & \vec{r}_{\mathrm{spot}}=(-0.287,-0.0877) \cdot a \\ & I_{1}=15000 \\ & I_{2}=-1.7 \\ &$\hline \hline\end{aligned}

The accretion disk is modeled as being circular symmetric around the white dwarf. In general, the disk height for a given distance $r$ from the white dwarf is calculated using the parameters $h_{1}$ and $h_{2}$ :

$$
h(r)=h_{1} \cdot\left(\frac{r}{r_{\text {disk,out }}}\right)^{h_{2}}
$$

Note that the thickness of the disk is $2 h$, since $h$ describes the height measured from the orbital plane. One typically distinguishes the following types of disks: The flat disk, where $h_{1}>0$ and $h_{2}=0$; the flared disk, with $h_{1}>0, h_{2}=1$; and the concave disk, where $h_{1}>0, h_{2}>1$.

Classical eclipse mapping codes always assumed flat disks, which is clearly justified by the short phase interval used by eclipse mapping and valid for the assumed physical models. If the whole orbit is used, the effects of a non-flat disk have to be taken into account. In Doppler mapping, no assumption about the disk geometry is made at all.

\subsection{Test calculations}

Similarly to the tests of the eclipse mapping code (Sect. 4.4, p. 32) and the FOEM method (Sect. 5.2, p. 47), I calculate artifical spectra of systems with a known geometry and intensity distribution. I add noise to these synthetic data sets and try to reconstruct the initial intensity distribution. Such tests are essential to understand the properties 
of the results created by the orbital mapping method with real data. While I have done an extensive amount of tests, I will show the results of two different setups. The first setup, described in Sect. 6.2.1, tries to find the limit of the reconstruction method by using intensity distributions which will be impossible - or at least very improbable - in a real CV. Values like the spatial resolution or the point spread function of the orbital mapping method can be estimated from such setups. The second type of tests (Sect. 6.2.2) tries to model typical spectra from real CVs. With those input data we can estimate which artifacts are to be expected when looking at observed spectra.

For the test, I calculated 40 spectra with an equal separation in orbital phase. The emission line FWHM was chosen to be $65 \mathrm{~km} / \mathrm{s}$. Each spectrum contains the intensities for 100 different velocities (wavelength intervals), each $100 \mathrm{~km} / \mathrm{s}$ wide. In the following figures, the spectra are shown as "trailed spectrograms", with a negative grayscale. The orbital phase runs from the bottom to the top, for clarity, two full orbits are shown. The spectrum denominated "observed" is the synthetic data set which is the target spectrum for the reconstruction.

\section{Excursion: Synthetic noise}

To simulate a typical observation run, I noisify the synthetic spectra using the following formula:

$$
I_{\text {noise }}(\phi, v)=\underbrace{f_{1} z_{1}(\phi, v)}_{\text {instrumental noise }}+I(\phi, v)(1+\underbrace{\left(1+\left|f_{2} z_{2}(\phi)\right|\right)}_{\text {clouds }} \cdot \underbrace{f_{3} z_{3}(\phi, v)}_{\text {photon noise }}) .
$$

Here, $z_{i}$ are Gaussian distributed random numbers which are either chosen differently for each velocity interval in each phase interval $\left(z_{1}, z_{3}\right)$ or the same for all velocity intervals at a given phase, but differently from phase to phase $\left(z_{2}\right)$. The first term in Eq. (6.11) simulates a constant instrumental noise $\left(f_{1}\right)$. The second term simulates noise which is of different strength for each phase interval intended to account for different observing conditions (clouds, $f_{2}$ ). The general noise level is $f_{3}$. For the test I show here, I choose

$$
\begin{array}{llll}
f_{1} \approx \frac{1}{100} I_{\max } & f_{2}=3 & f_{3}=0.01 & \text { or } \\
f_{1} \approx \frac{1}{20} I_{\max } & f_{2}=3 & f_{3}=0.05
\end{array}
$$

This compares to $\mathrm{S} / \mathrm{N} \approx 30 \ldots 100$ or $\mathrm{S} / \mathrm{N} \approx 10 \ldots 20$, respectively, depending on the "cloud coverage". 


\subsubsection{Purely artifical test maps}

The system parameters of my test system $C V$ Toy are given in Tab. 6.1. The intensity for any point $\vec{r}$ on the disk is calculated by

$$
I(\vec{r})=\left(\frac{|\vec{r}|}{5 a}\right)^{I_{2}} \cdot I_{\text {pattern }} \quad I_{2}<0
$$

where the coordinate system of $\vec{r}$ has its origin at the center of the white dwarf. I used two different setups in which I tried to reconstruct very unphysical but insightful intensity distributions $I_{\text {pattern }}$.

\section{Six bright spokes}

The intensity pattern is choosen so that $I_{\text {pattern }}$ alternates between 1 and 10 every $30^{\circ}$. That leads to six spokes which are ten times brighter than the normal disk (Fig. 6.1, top left). The trailed spectrum reflects these spokes with six sinusoidal features, which mingle to a snake skin-like pattern (same Fig., bottom left).

The reconstructed spectrum (bottom right) shows the input features, but with much less contrast. As a result, the residuals (middle) also show the relevant features.

As one can see in the reconstructed intensity map (Fig. 6.1, top right), the general shape of the input map is produced correctly. The algorithm finds six equal spokes at the correct orbital positions. Only the intensity increase towards the center does not come out very well.

The fact that even in the residuals of the spectrograms much of the "observed" and the reconstructed data are visible can be important in the interpretation of fit results with real data.

\section{Four bright spots}

For this type of tests, $I_{\text {pattern }}$ again alters between 1 and 10 . Now the position with the high value are centered around $x / a=-0.2,0,0.2,0, y / a=0,0.2,0,-0.2$ and have a radius of $0.05 a$ (Fig. 6.2 , top left). The trailed spectrum shows four sine curves for the four spots (Fig. 6.2, bottom left).

The lower spatial frequence of the features in the trailed spectrum makes the reconstruction slightly easyier than for the six-spoked disk above. The calculated spectrum, which belongs to the reconstructed map shows only very slight deviations from the input spectrum (Fig. 6.2, top right, bottom right, bottom center). The increase in brightness toward the disk center, which is in the input map, is again not reconstructed well, while the position and size of the four bright spots are reproduced exactly. 

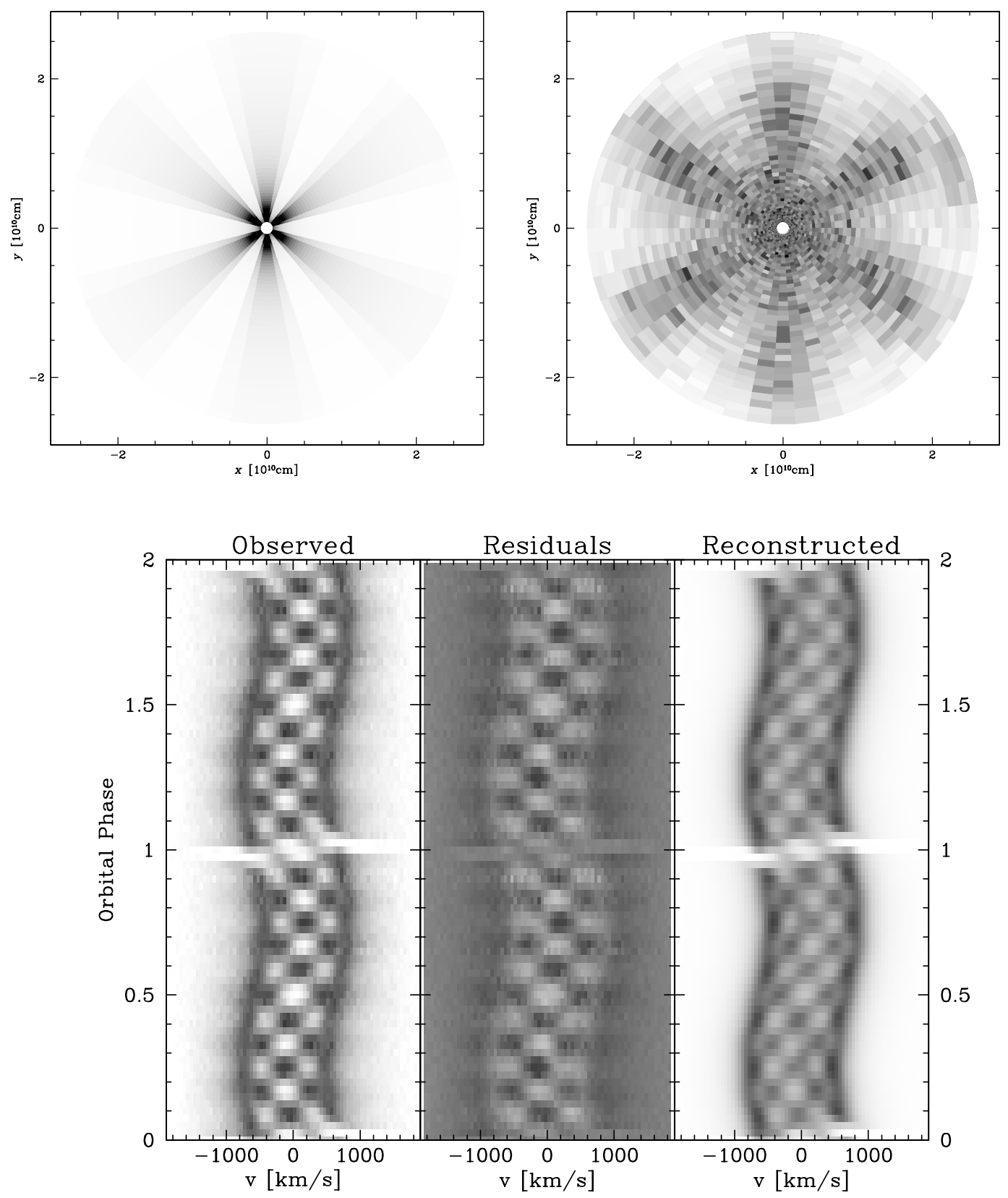

Figure 6.1: Top: Input and reconstruction map of the six-spoked star. Bottom: "Observed", i.e. synthetic, and reconstructed spectrum for the six-spoked star input map 

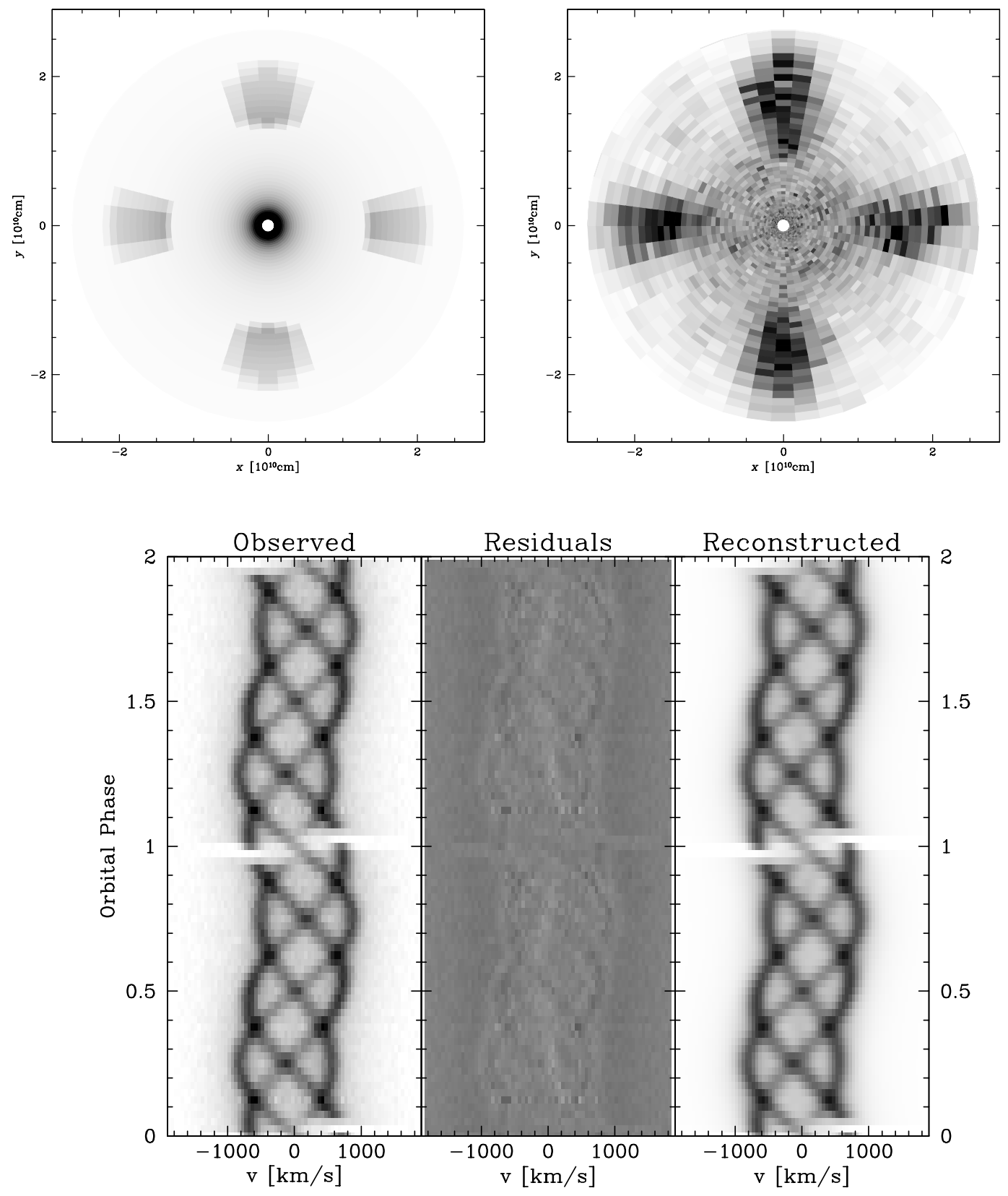

Figure 6.2: Top: Input and reconstruction map of the four spot model. Bottom: "Observed", i.e. synthetic, and reconstructed spectrum for the four spots input map

\subsubsection{Toy model simulations}

As a toy model of a $\mathrm{CV}$ with a disk, I calculated synthetic trailed spectra for a system with the parameters shown in Tab. 6.1. The intensity for every point $\vec{r}$ on the disk is calculated by

$$
I(\vec{r})=\left(\frac{|\vec{r}|}{5 a}\right)^{I_{2}}+I_{1} \cdot \exp \left(-\left[\frac{\left|\vec{r}-\vec{r}_{\text {spot }}\right|}{36 a}\right]^{2}\right)
$$


where the coordinate system of $\vec{r}$ has its origin at the center of the white dwarf, $+x$ points away from the secondary star, and $+y$ points in the direction of the orbital motion of the white dwarf. The factors 5 and 36 are arbitrary and determine the relative contribution of the spot and its size. This intensity distribution resembles a disk with a hot spot located where the ballistic accretion stream impacts on the disk and heats the impact region. The initial intensity distribution is shown in Fig. 6.3, top left.

The "toy model" spectrum was noisified with a $\mathrm{S} / \mathrm{N} \approx 100$ as in Eq. (6.12a) and with a $\mathrm{S} / \mathrm{N} \approx 10$ as in Eq. (6.12b). The noisified spectra together with the reconstructed maps and their spectra are shown in Figs. 6.3 and 6.4, respectively. It is amazing, how well the algorithm is able to find the position, size, and contrast of the model hot spot in both the high-quality and low-quality input data. As before with the "four spots" test, the increase of the disk brightness towards the center of the disk does not come out perfectly.

\section{Radial profile}

Since the radial intensity profile is of interest for considerations of the disk structure, it is desireable, if an image reconstruction algorithm can give reliable results on the intensity behaviour towards the center. The orbital mapping algorithm as shown in the four tests above does not, but a slight enhancement of the fitting program biases the results towards a very successful radial intensity reconstruction.

The idea is not only to fit the surface elements, but also multiply them by a factor which depends on the distance from the WD. If $I_{i}^{\prime}$ is the preliminary intensity of the $i$-th surface element, then the intensity actually used is

$$
I_{i}=I_{i}^{\prime} \cdot \alpha r^{-\beta}
$$

with $r$ being the distance from the surface element to the white dwarf. Now not only the $N$ different surface elements are fitted, but also two more values, $\alpha$ and $\beta$.

The maps which result from this modified algorithm appear somewhat more speckled (Fig. 6.5, top right) than in the unmodified algorithm, but if one looks at a trace through the disk one finds that the radial symmetric component of the intensity comes out nearly perfectly even in the innermost parts of the disk (Fig. 6.6).

The fact that even in the modified map the innermost parts of the disk seem to have a slightly flatter intensity increase than the outer parts are a direct result of the entropy which is used here, that on purpose does not take into account the radial symmetry of the disk.

The need for a parametrization of the disk profile is imposed by the evolution strategy. The fit algorithm is able to reproduce contrasts of up to 1:20 or 1:30. Since the inner 

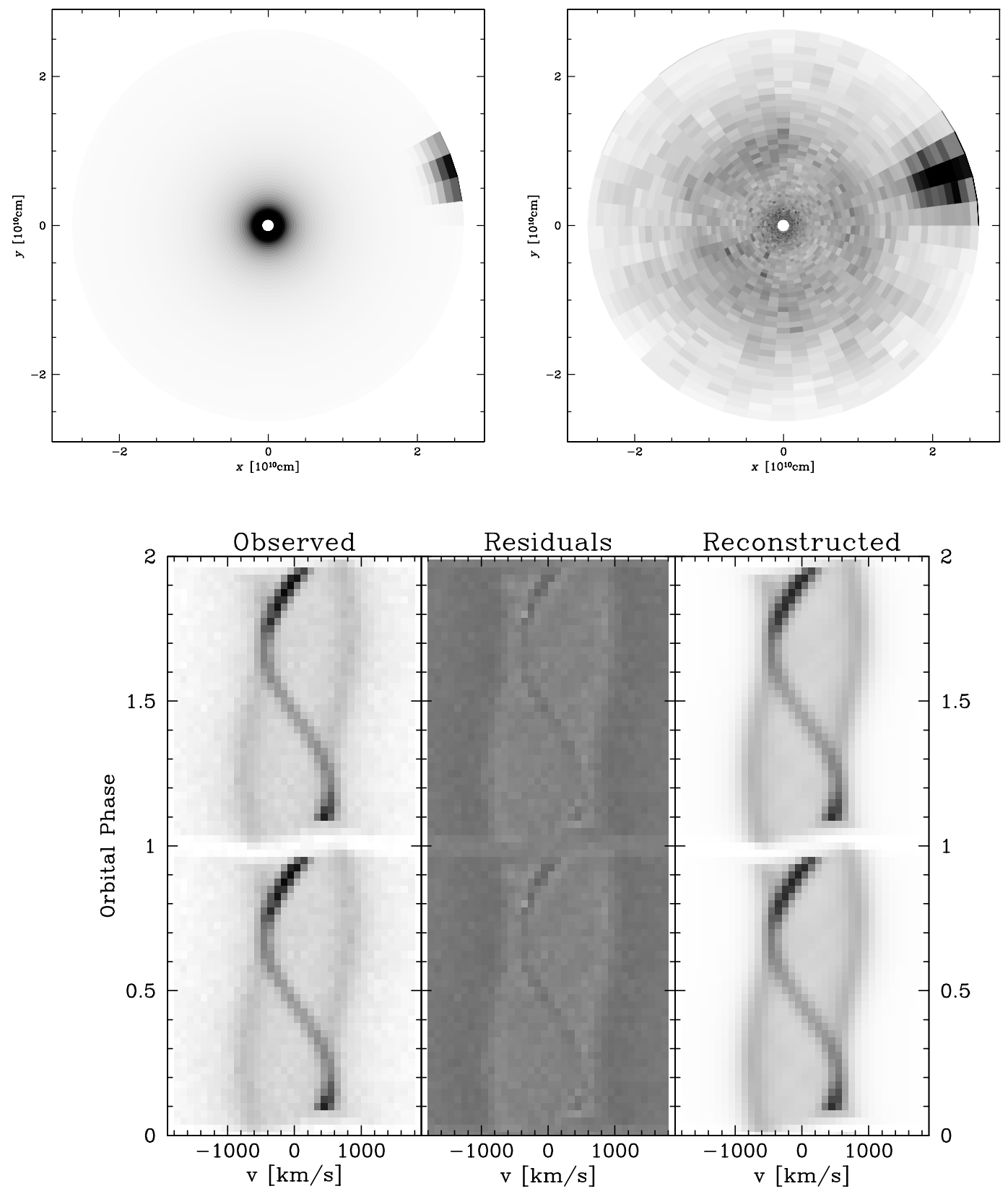

Figure 6.3: Top: Input and reconstruction map of the "toy model". Bottom: "Observed", i.e. synthetic, reconstructed spectrum, and residuals for the toy model input map.

disk regions are 1000 to 10000 times as bright at the outer regions, one has to help the fit algorithm as described. 

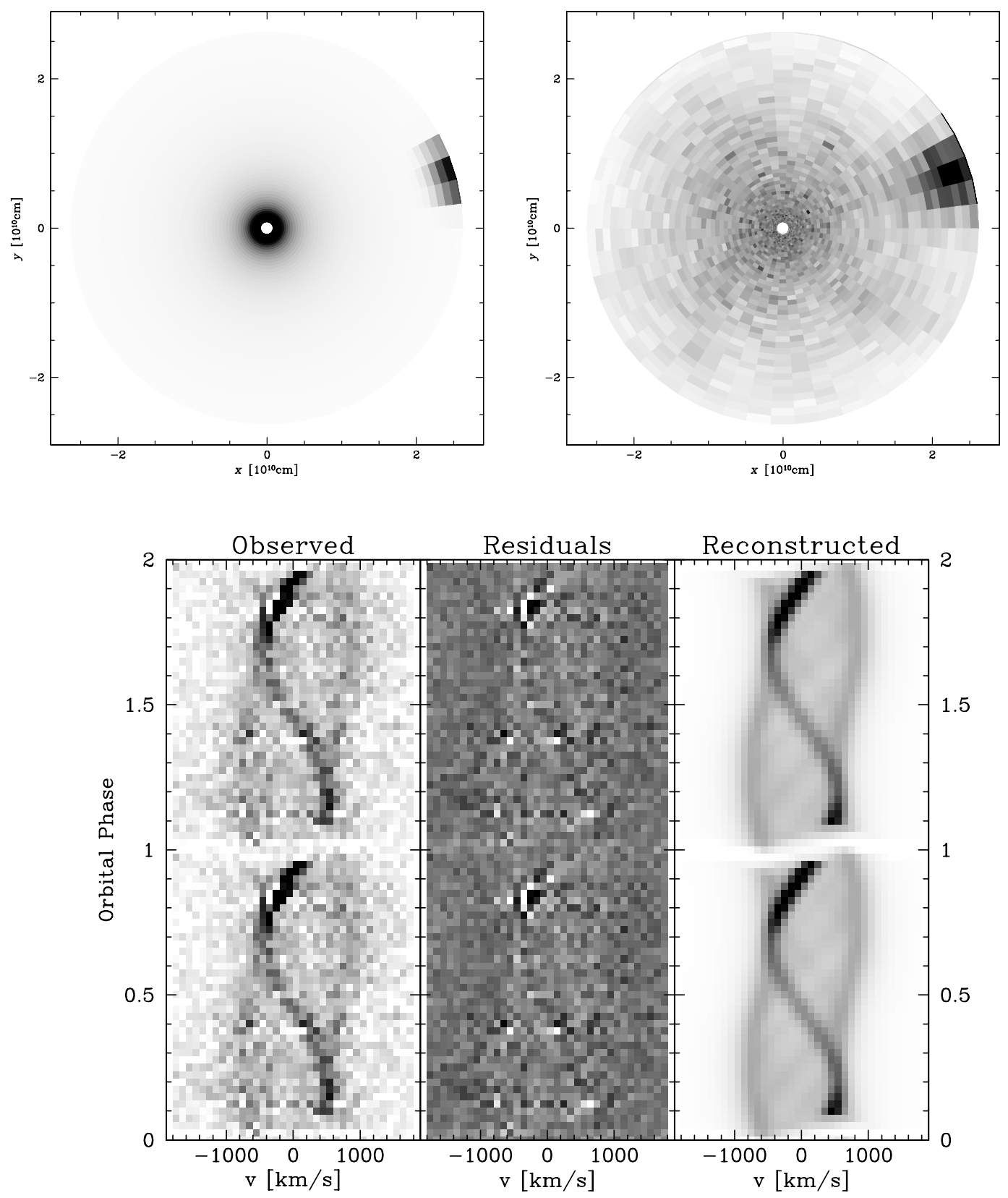

Figure 6.4: Top: Input and reconstruction map of the "toy model". Bottom: "Observed", i.e. synthetic, reconstructed spectrum, and residuals for the toy model input map, $\mathrm{S} / \mathrm{N} \approx 10$.

\section{Incorrect geometry}

As a last test, I tried to reconstruct the toyspot map with a slightly incorrect geometry. The resulting maps and the light curves are shown in Figs. 6.7 (high $\mathrm{S} / \mathrm{N}$ ) and 6.8 (low $\mathrm{S} / \mathrm{N})$. The incorrect geometry involves a mass ratio of $M_{1} / M_{2}=2.5$ instead of 1.4 . 

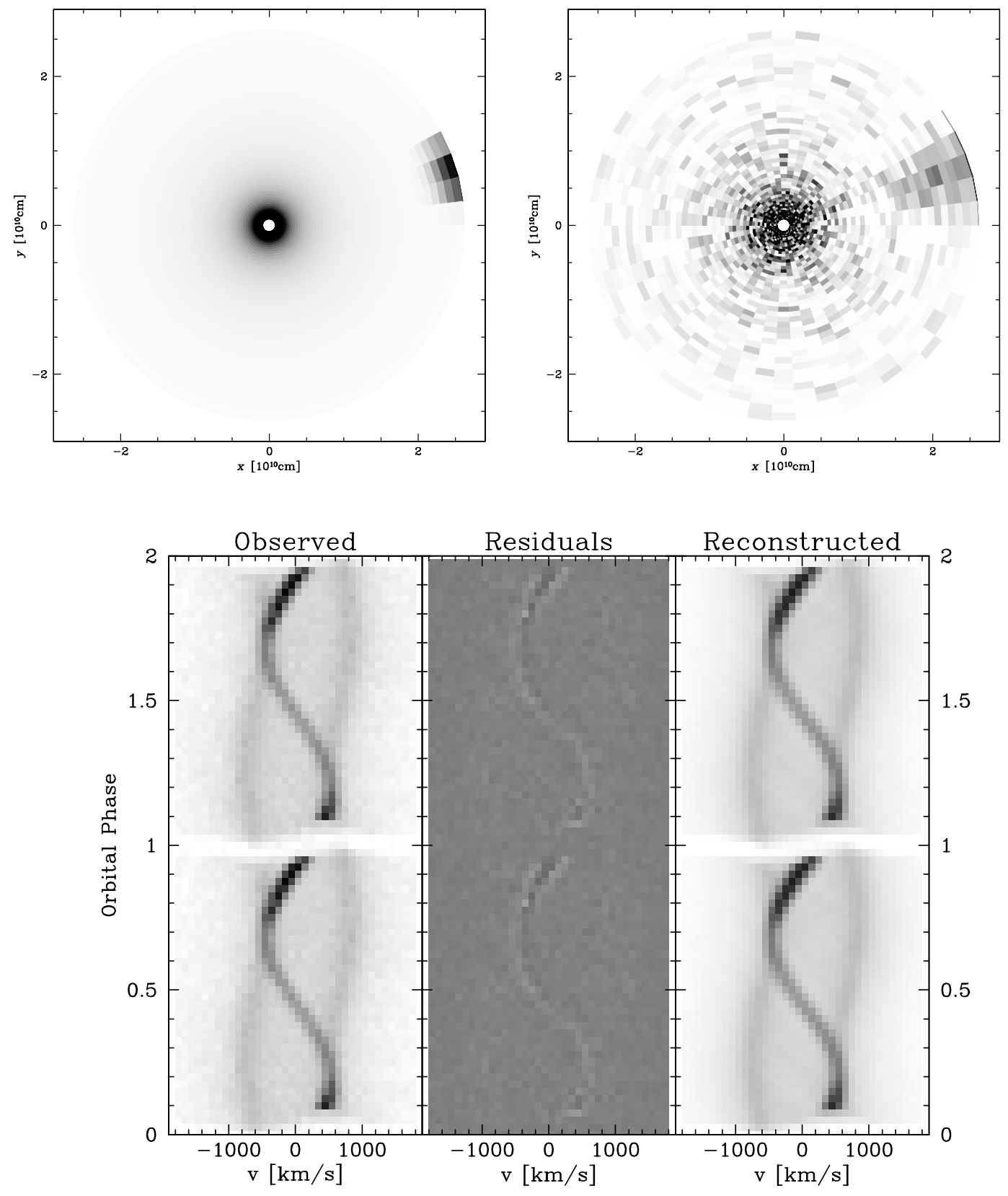

Figure 6.5: Top: Input and reconstruction map of the "toy model". Bottom: "Observed", i.e. synthetic, reconstructed spectrum, and residuals for the toy model input map, $\mathrm{S} / \mathrm{N} \approx 100$. A radial symmetric component $\propto r^{-\alpha}$ is fitted as well.

That leads to an slightly higher inclination, $i=79^{\circ}$ instead of $75^{\circ}$. In order to take into account the different Kepler velocity of the disk rim $(514 \mathrm{~km} / \mathrm{s}$ in the input geometry), the disk has been made larger $(0.4 \cdot a$ instead of $0.3 \cdot a)$, which still leads to a slight offset in the Kepler velocity, which is $501 \mathrm{~km} / \mathrm{s}$ now. 

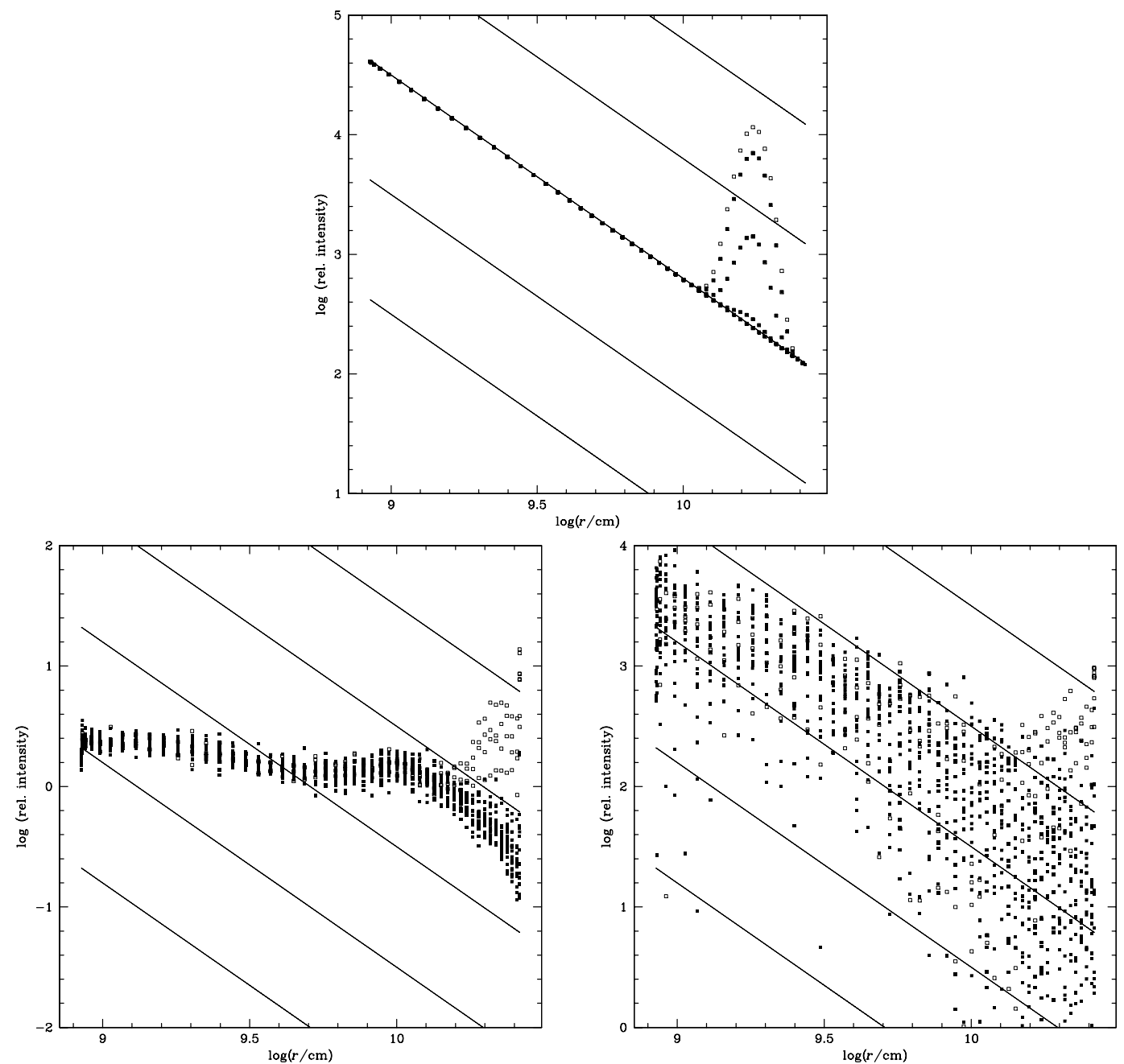

Figure 6.6: Traces trough the toy model disk. Top: input disk, bottom left: reconstruction with no radial symmetric intensity parameter as fit option, bottom right: reconstruction with an radial symmetric fit parameter. The open boxes mark points which pass through the region of the hot spot, the points mark all other points. The lines show the input intensity profile $I \propto r^{-1.7}$.

The resulting reconstructions show no significant deviations in the bright spot position from the maps reconstructed with the correct geometry. A slight increase in the general disk noise with some additional low-contrast features in the disk is detectable. The difference plot of the input and the reconstructed trailed spectra shows a less suitable fit of the reconstructed data to the synthetic input data than for the correct geometry. Nevertheless, the method seems to be robust against slight changes in the system parameters, if the parameters fit to the general features (Kepler velocity of the disk $\operatorname{rim}, i, q$-value). If the data used for mapping are of sufficiently high quality $(\mathrm{S} / \mathrm{N} \gtrsim 50$ ), the features in the resudual trailed spectrum can in principle be used to fix the system 

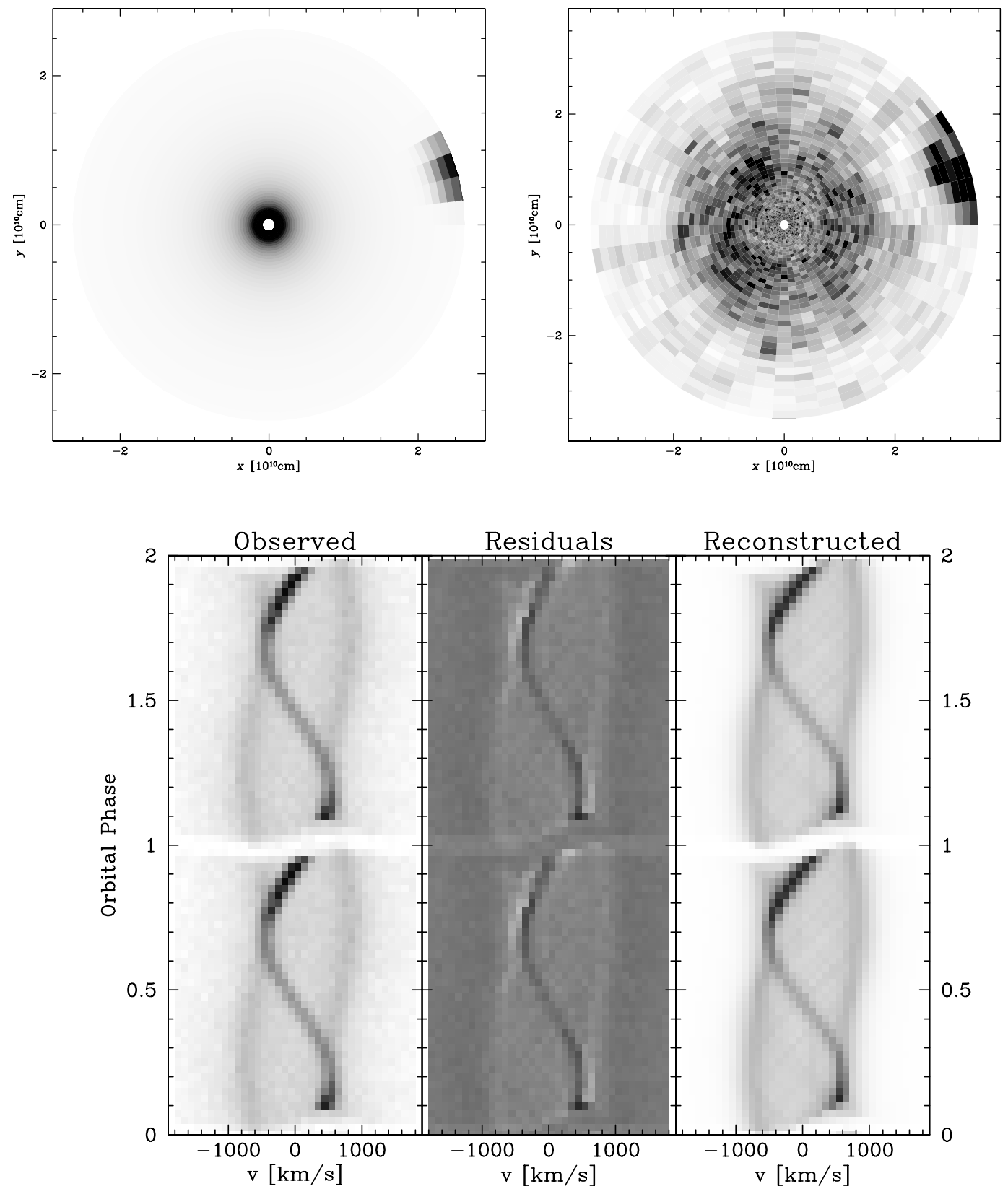

Figure 6.7: Top: Input and reconstruction map of the "toy model". Bottom: "Observed", i.e. synthetic, reconstructed spectrum, and residuals for the toy model input map, $\mathrm{S} / \mathrm{N} \approx 100$. The assumed geometry is not identical to the original geometry.

parameters. That application, however, has not yet been applied due to the lack of suitable data.

This test showed that the OMA algorithm is robust agains small deviations between the assumed and the actual geometry of the mapped object. 

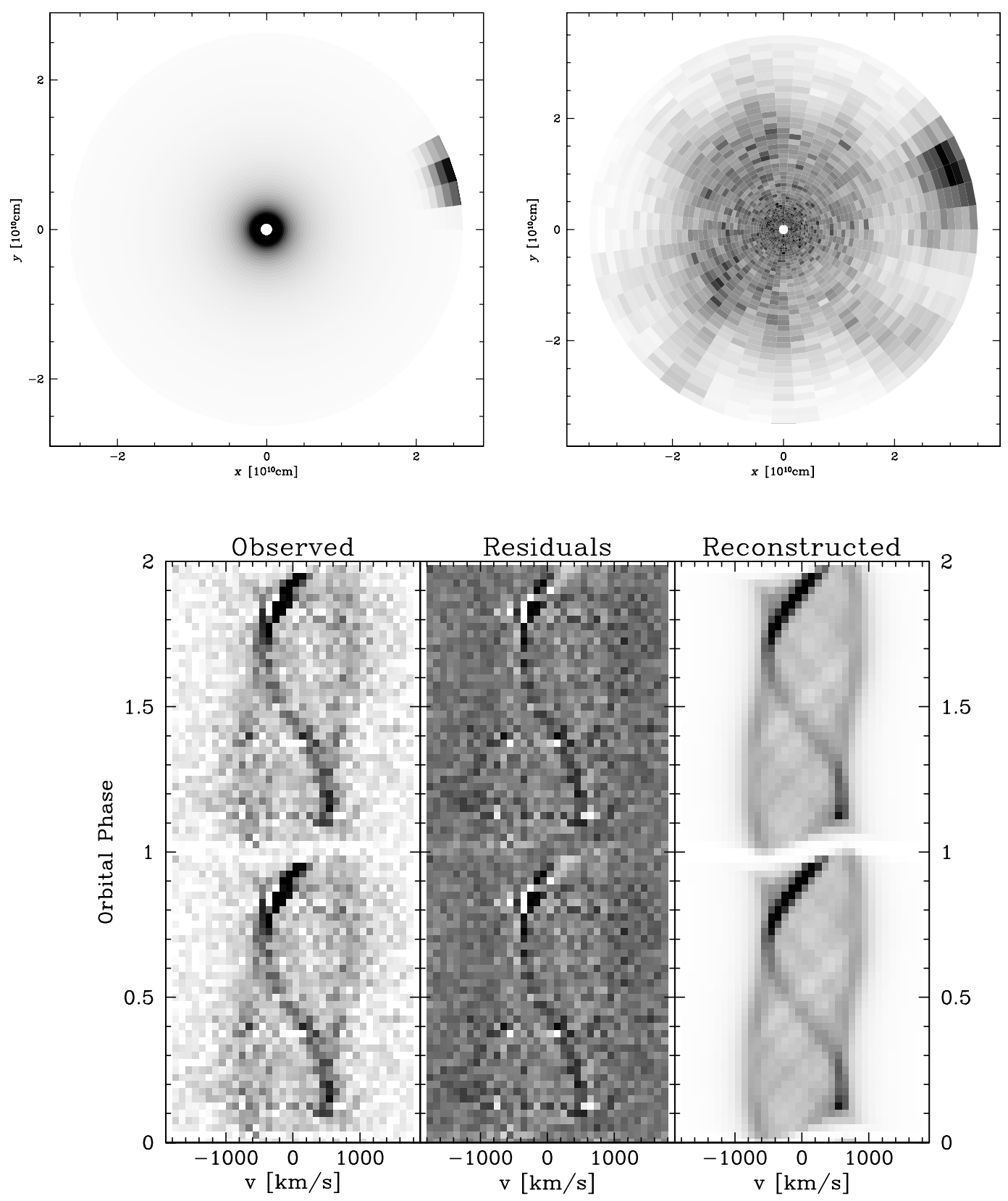

Figure 6.8: Top: Input and reconstruction map of the "toy model". Bottom: "Observed", i.e. synthetic, reconstructed spectrum, and residuals for the toy model input map, $\mathrm{S} / \mathrm{N} \approx 10$. The assumed geometry is not identical to the original geometry. 


\section{$6.3 \quad \mathrm{HS} 0455+8315$}

For the first application of the orbital mapping method, I choose HS0455+8315, which I observed with two telescopes at the Calar Alto observatory, Spain, in December 2000/January 2001. In this section, I describe the classical analysis of the data, the next section contains the attempt to map HS0455+8315 with OMA.

HS0455+8315, abbreviated as HS0455 from now on, is a newly discovered CV (Gänsicke et al., 2002b). It was found in the Hamburg Quasar Survey (Hagen et al., 1995) as a CV candidate and confirmed in follow-up observations by Gänsicke \& Nogami (Calar Alto, 2.2m CAFOS-spectroscopy), Schwarz, Staude \& Engels (time resolved photometry, $70 \mathrm{~cm}$ telescope in Potsdam), and Kube \& Kuduz (high-resolution phase resolved spectroscopy at the Calar Alto 3.5m telescope with the TWIN spectrograph, simultaneous time resolved photometry at the Calar Alto $1.23 \mathrm{~m}$ telescope).

HS0455 was found to show deep eclipses (Fig. 6.9). The eclipse does, however, not show a flat bottom, but a $\mathrm{V}$ shape, indicating that the disk is not totally eclipsed. This finding is supported by the width of the primary eclipse, which can be measured as $\Delta \phi=0.075$, indicating an inclination of less than $\approx 80^{\circ}$, depending on the mass ratio used. The spectra taken during the center of the eclipse still show emission lines (Fig. 6.13), giving the final evidence for a grazing eclipse of the accretion structure.

From the eclipse timings, we were able to derive an orbital ephemeris:

$$
\mathrm{HJD}=2451859.2446(2)+0.148728(1) \cdot N
$$

This implies an orbital period of

$$
P=214.168(2) \min
$$

which positions HS0455 just in the middle the period range of the known SW Sex systems.

Apart from the eclipse, little orbital variation is seen in the continuum light curves: There is a very shallow and broad secondary minimum around $\phi \approx 0.5$ which coincides with the emission line minimum as will be shown below. Due to the deep eclipse, it is not possible to distinguish whether the orbital variation is single or double humped, since a possible second minimum at around $\phi \approx 0.0$ is dominated by the eclipse. The emission line behaviour, which clearly shows two maxima around the orbit, is in favour of a double humped lightcurve (Fig. 6.19).

HS0455 shows no emission in the X-Ray range as seen in the ROSAT all sky survey. EUVE UV observations do not show a signal from HS0455 either.

HS0455 is clearly detected in the $\mathrm{K}$ and $\mathrm{J}$ images of the 2MASS, while the $\mathrm{H}$ band image of that region seems to be overexposed and is unusable. 


\section{Excursion: Measurement of the eclipse width}

Warner (1995b) states, that one can measure the eclipse width $\Delta \phi$ of a system as the width at half depth, $\Delta \phi_{1 / 2}$, if the eclipse of the primary component is not identifyable. While this is true for systems with $i \gtrsim 80^{\circ}$ the method is not accurate if the eclipse of the disk is grazing. Errors of up to $2 \ldots 3^{\circ}$ in the measurement of $i$ with a known mass ratio $Q$ could be the result (Tab. 6.2). From my simulation of eclipse profiles, I find that if $\Delta \phi_{1 / 2}<0.08$ one should be careful and apply a correction:

$$
\Delta \phi= \begin{cases}\Delta \phi_{1 / 2} & \text { if } \Delta \phi \gtrsim 0.080 \\ \Delta \phi_{1 / 2}-0.001 \ldots 0.002 & \text { if } \Delta \phi \lesssim 0.080\end{cases}
$$

For HS0455, $\Delta \phi=0.075$ should be a good value.

Table 6.2: Eclipse widths of artifical systems. For systems with grazing eclipses, the width $\Delta \phi$ cannot be measured as the width at half depth, $\Delta \phi_{1 / 2}$. The real $\Delta \phi$ is smaller than $\Delta \phi_{1 / 2}$.

\begin{tabular}{ccccc}
\hline \hline given $Q$ & given $i$ & real $\Delta \phi$ & measured $\Delta \phi_{1 / 2}$ & derived $i$ \\
\hline 2.0 & $72^{\circ}$ & n/a & 0.047 & $74^{\circ}$ \\
2.0 & $75^{\circ}$ & 0.053 & 0.055 & $76^{\circ}$ \\
2.0 & $80^{\circ}$ & 0.082 & 0.082 & $80^{\circ}$ \\
\hline \hline
\end{tabular}

The integrated spectrum of HS0455 as observed with the Calar Alto $3.5 \mathrm{~m}$ telescope and the TWIN two-channel spectrograph over 1.15 orbits $(246 \mathrm{~min}$, of which the shutter was open for $215 \mathrm{~min}$ ) is shown in Figs. 6.10 (red and blue part in one graph), and 6.11 (both parts separately). The spectra were not corrected for orbital motion. The flux calibration of the TWIN-spectra was made with the simultanous photometric data, obtained at the $1.23 \mathrm{~m}$ telescope. The TWIN was used with the T05 grid (blue channel) and the T06 grid (red channel), both with 1200 lines/mm in the first order dispersion. With a 1.5" slit, the spectral resolution (measured from the calibration lines) is $\mathrm{FWHM}=1.32 \AA$ in the blue and FWHM $=1.23 \AA$ in the red spectrum. The instrumental velocity resolution then is

$$
\begin{array}{ll}
\mathrm{FWHM}_{4686}=85 \mathrm{~km} \mathrm{~s}^{-1} & \text { at He II } \lambda 4686 \\
\mathrm{FWHM}_{6563}=56 \mathrm{~km} \mathrm{~s}^{-1} & \text { at H } \alpha
\end{array}
$$


Table 6.3: Eclipse data of HS0455

\begin{tabular}{rc}
\hline \hline Orbit No & $\phi_{0}-2,450,000$ \\
\hline 0 & 1859.24469 \\
1 & 1859.39312 \\
41 & 1865.34143 \\
168 & 1884.2305 \\
359 & 1912.63820 \\
\hline \hline
\end{tabular}

The slope of the continuum in the spectrum is in good agreement with the prediction from Lynden-Bell and Pringle (1974), who claim that the continuum emission from a steady-state accretion disk behaves like

$$
F_{\lambda} \propto \lambda^{-7 / 3}
$$

This relation is shown in the figures by the dashed line. Since Eq. (6.20) is valid only for infinitely large disks, the agreement with our observations may be incidentally.

The spectrum shows strong emission lines, which are the hydrogen Balmer series, neutral and ionized helium lines, oxygen, nitrogen, and carbon lines. All identified emission lines are listed in Tab. 6.4. The absorption features at $\lambda \approx 6280 \AA$ and $6870 \AA$ are from the Earth's atmosphere.

From the light curve, the emission line structure, and the Doppler tomogram, we can conclude, that HS0455 belongs to the class of SW Sex stars (Hoard, 1998).

\subsubsection{The emission lines}

HS0455 shows two different behaviours of its emission lines. The lines of neutral hydrogen and helium show double peaks in the integrated spectrum, indicating their origin in an accretion disk or similar object. As will be seen later, the double-peak nature of the neutral lines is not genuine, the depleted zero velocity flux is the result of an absportion feature. The lines of ionized elements are single peaked. This behaviour is typical of SW Sex systems.

In Fig. 6.12, all four observed hydrogen emission lines are plotted in one graph. While the offset between the four continua shows, that the $F_{\text {disk }} \propto \lambda^{-7 / 3}$ is not a perfect approximation for the continuum emission of the disk, another striking feature appears: The blue peak of the double emission line increases in strength compared to the red peak with decreasing wavelength, indicating a higher temperature of the blue part of the emission line. This can be understood, if one looks at the trailed spectrum: The blue and the red line peak in their intensities at opposite orbital phases. The interpretation 


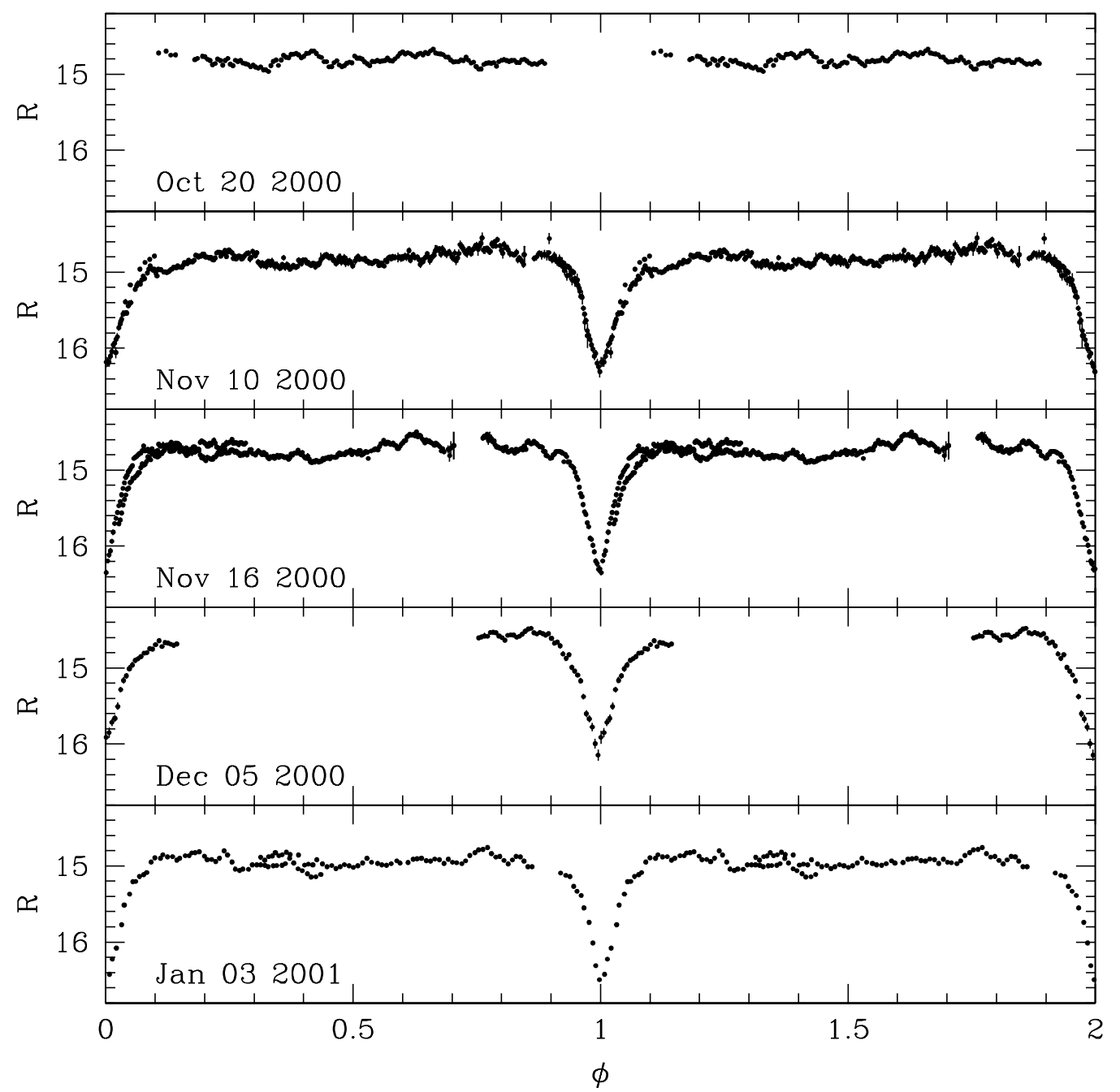

Figure 6.9: The five individual R light curves of HS0455 obtained so far. Note that the eclipse was just missed at the first observation run. The light curve from January 3 was taken at the Calar Alto $1.23 \mathrm{~m}$, all others at Potsdam.

is this: The bright peak in the double peaked hydrogen lines is emitted by a two-sided optically thick region. The side visible at $\phi \approx 0.75$ has a higher temperature than the side visible at $\phi \approx 0.25$.

The strongest single peaked line (He II $\lambda$ 4686) and the double peaked $\mathrm{H} \alpha$ line, which has the best signal-to-noise ratio of the hydrogen lines, are analyzed in detail: I take a closer look at the eclipse behaviour, the orbital variation, and the classical doppler tomograms of these lines. Then I present the orbital mapping results for the $\mathrm{H} \alpha$ line. 


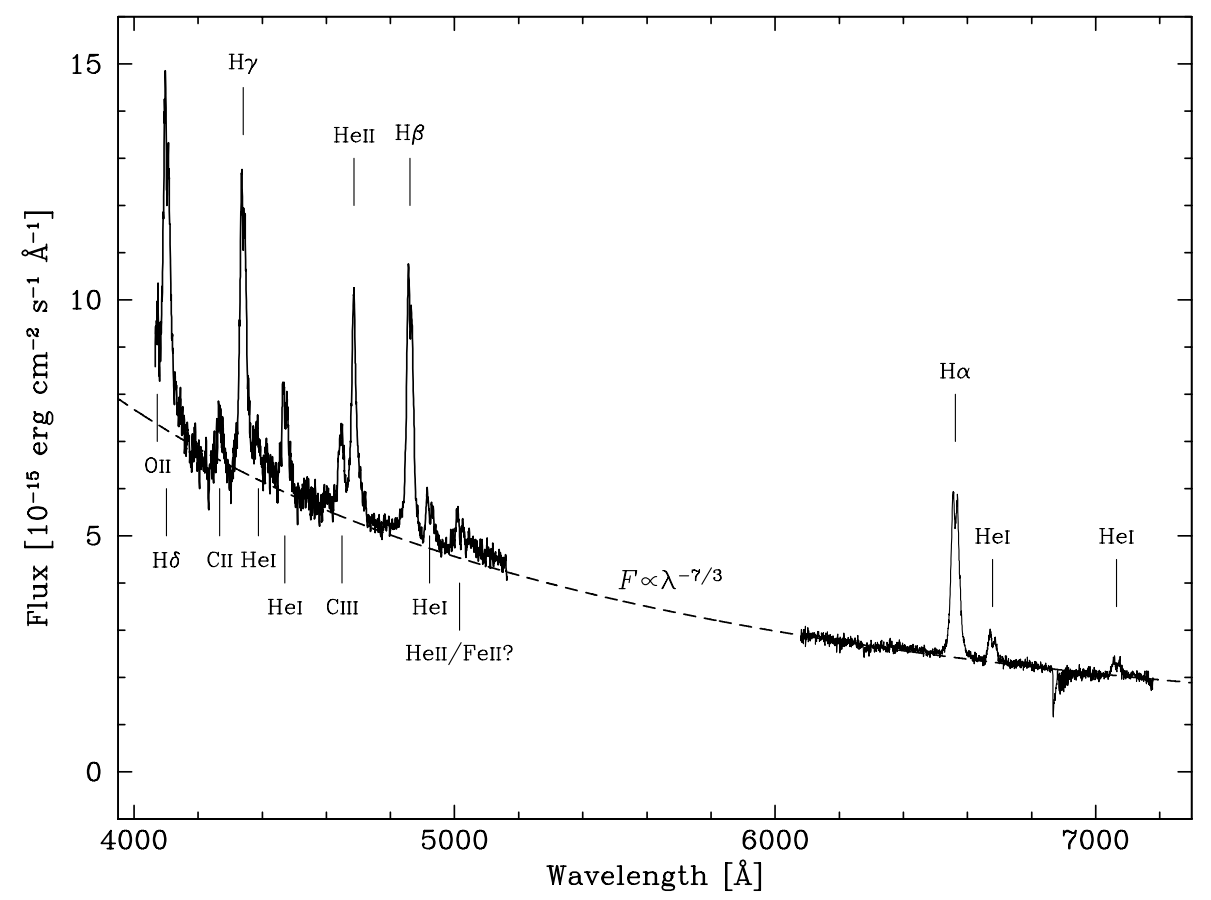

Figure 6.10: Calar Alto TWIN-spectrum of HS0455+83. Average of 33 exposures with 215 min total exposure time. A power law is used to indicate the approximate position of the continuum.

\section{Eclipse behaviour}

The behaviour of the two analysed lines during the eclipse can be seen in Figs. 6.13 and 6.14. In the first of these, the lines of $\mathrm{H} \alpha$ are shown in the range $\phi=0.8134 \ldots 0.1854$, in the second, the HeII $\lambda 4686$ emission is shown at the center of the eclipse (one exposure, hence the bad $\mathrm{S} / \mathrm{N}$ ), at the rising part of the eclipse (average of three exposures, $\phi=0.0534 \ldots 0.1177$ ) and integrated far outside the eclipse (9 exposures at $\phi=0.3514 \ldots 0.6410)$. The eclipse in the continuum light starts at $\phi=0.89$ and ends at $\phi=0.11$.

The analysis of the eclipse behaviour of $\mathrm{H} \alpha$ is complicated by the orbital variation of the line, which would lead to a decrease of the red peak of the emission, even if no eclipse were present. Subtracting that trend of the strong red peak, the following features are found: At the beginning of the eclipse, first the blue emission decreases slightly until at the center of the eclipse a broad base component with a smaller peak at $v=210 \mathrm{~km} / \mathrm{s}$ is left. The small red peak is part of the structure that forms the large red peak, but is not eclipsed. After the eclipse, first the blue side of the spectrum starts to reappear, while the increase of the red line back to a large intensity follows $\approx 0.02$ later in phase. 

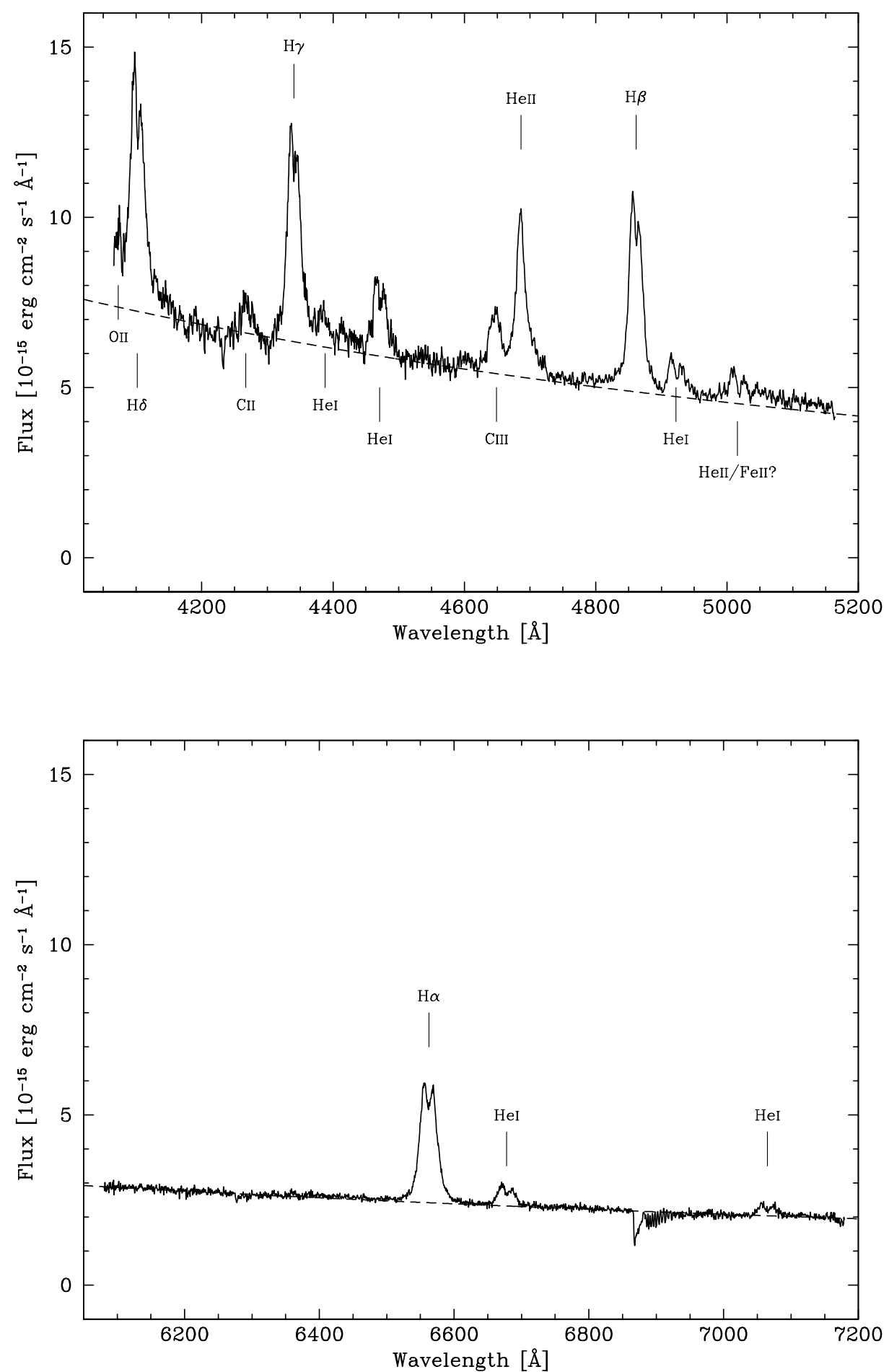

Figure 6.11: Blue (top) and red (bottom) part of the Calar Alto TWIN-spectrum of HS0455+83. Average of 33 exposures with 215 min total exposure time. 
Table 6.4: Emission lines found in the HS0455-spectrum

\begin{tabular}{|c|c|c|}
\hline Wavelength/A & Species & Behaviour \\
\hline 4101.73 & $\mathrm{H} \delta$ & "double peaked" \\
\hline 4340.46 & $\mathrm{H} \gamma$ & "double peaked" \\
\hline 4861.33 & $\mathrm{H} \beta$ & "double peaked" \\
\hline 6562.79 & $\mathrm{H} \alpha$ & "double peaked" \\
\hline 4388 & He I & weak, single peaked? \\
\hline 4471 & He I & "double peaked" \\
\hline 4686 & He II & single peaked \\
\hline 4922 & He I & "double peaked" \\
\hline 5016 & He II/Fe II? & weak \\
\hline 6678 & He I & "double peaked" \\
\hline 7065 & He I & "double peaked" \\
\hline 4073 & O II & in the wing of $\mathrm{H} \delta$, single peaked \\
\hline 4267 & C II & single peaked \\
\hline 4649 & $\mathrm{C}$ III/N III & single peaked \\
\hline
\end{tabular}

The diagnosis of the He II $\lambda 4686$ emission is affected by a $\mathrm{S} / \mathrm{N}$ which is much worse than that of the $\mathrm{H} \alpha$-line. Hence, time resolution during the eclipse has to be sacrificed in order to improve the S/N. In Fig. 6.14, one can clearly see that He II $\lambda 4686$ is not affected by the eclipse as strongly as the hydrogen lines. During the center of the eclipse, the line appears to be narrower than outside of the eclipse, but the maximum flux of the lines is always at $\approx 2.5 \times 10^{-15} \mathrm{erg} \mathrm{cm}^{-2} \mathrm{~s}^{-1} \AA^{-1}$. So, if some of the He II $\lambda 4686$ is eclipsed by the secondary star, it is a region producing broad emission.

\section{Orbital variation}

The orbital variation of the HS0455 spectrum includes the following features: (a) the eclipse, treated above; (b) the orbital motion of the system; (c) the behaviour of the emission line strengths and the position of their peaks.

The orbital motion of the single peaked He II $\lambda 4686$ line is straightforward to analyze. I fitted a single Gaussian to the line profile for each observed spectrum using the wavelength range which is not contaminated by the neighbouring $\mathrm{C}$ III/N III emission. The center of the Gaussians and their statistical errors are plotted in Fig. 6.15. To these, I fitted a sine curve, which yields

$$
V_{\text {He II } \lambda 4686}(\phi)=\underbrace{14(8) \mathrm{km} \mathrm{s}^{-1}}_{\gamma}-\underbrace{138(11) \mathrm{km} \mathrm{s}^{-1}}_{K} \cdot \sin \left(2 \pi(\phi-\underbrace{0.165(12)}_{\phi_{0}})\right)
$$




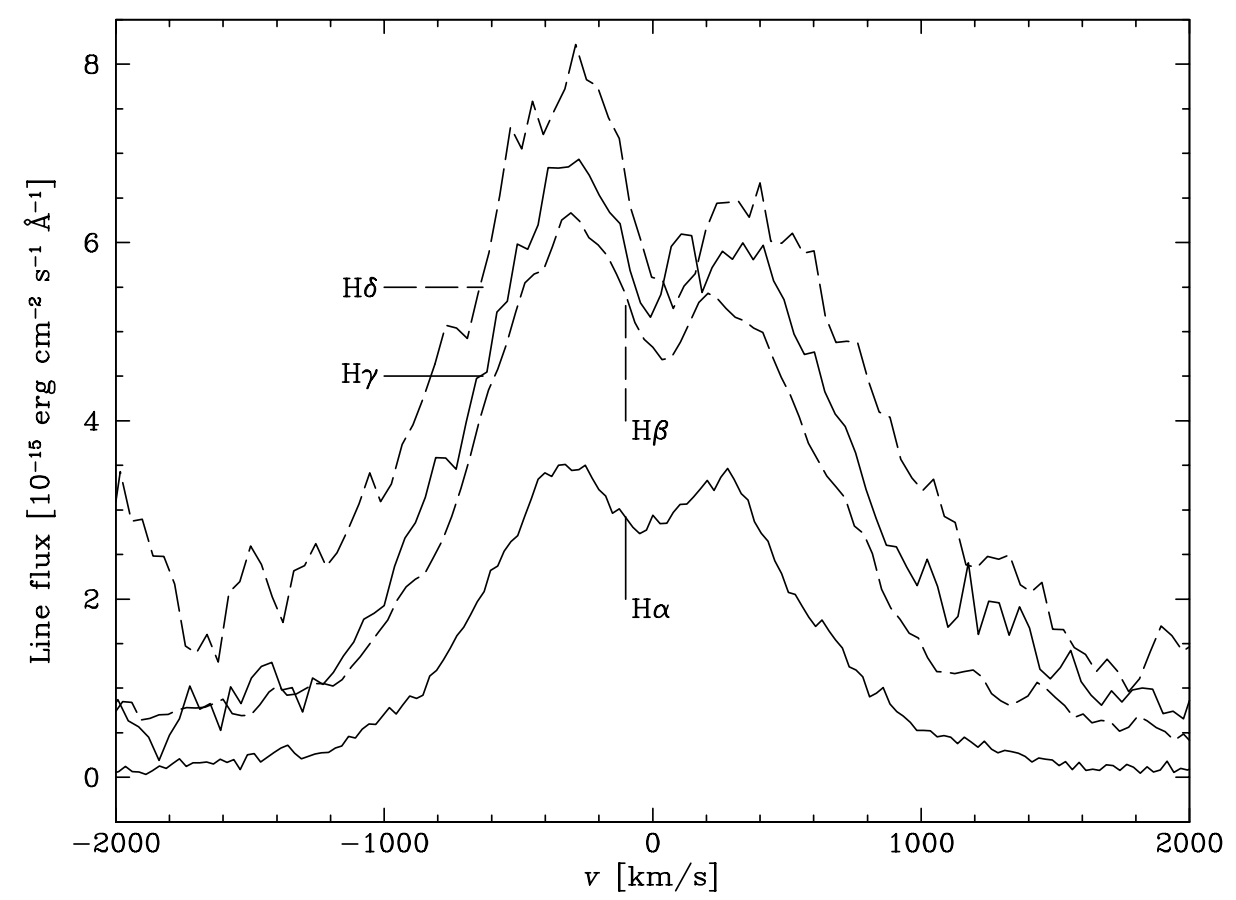

Figure 6.12: The hydrogen emission lines in HS0455, the wavelength displayed in velocity units. The line at $v=-2000 \mathrm{~km} / \mathrm{s}$ in the $\mathrm{H} \delta$-plot is $\mathrm{O}$ II $\lambda 4073$. Average of 33 exposures with 215 min total exposure time; continuum $F_{\text {disk }} \propto \lambda^{-7 / 3}$ subtracted. Note the increasing strength with decreasing wavelength of the blue peak compared to the red peak.

with the statistical errors in braces. This is very similar to the values found for the system BH Lyn, where Hoard and Szkody (1997) measured $K=125(8)$ and $\phi_{0}=$ $0.20(1)$. BH Lyn is in most respects very similar to HS0455, as will also be shown in the following section about Doppler tomograms of HS0455.

From earlier work on SW Sex systems, it is understood that the orbital velocity curves can only be of very limited diagnostic value when it comes to the system parameters (Hellier, 1996). There is a phase difference between the orbital motion of the white dwarf and an associated wind from the accretion disk - which would be characterized by $\phi_{0}=0$ in Eq. (6.21) - and a second component from an accretion stream re-impact after an overflow over the accretion disk at $\phi_{0} \approx 0.4$ (Fig. 6.16, left); the addition of both components would result in the observed $\phi_{0}$. Since the relative strength of both sources - wind and stream re-impact - are very difficult to derive in a unique way, and the "double peaked" lines consist of even more components (Fig. 6.16, right), I forego an orbital velocity analysis of the $\mathrm{H} \alpha$ line. 


\section{Excursion: Optically thick emission}

Two of the features found in the spectrum of HS0455 clearly justify or even require the optically thick approach for the orbital mapping analysis method for at least one of the mapped components. (1) The emission line light curve is not constant over the orbit. That means, that there are parts in the system which appear at a different brightness if seen from different viewing angles. That is in sharp contrast to the Doppler mapping assumption, where - by definition - the system brightness in the emission line is constant (Eq. (3.11)). (2) The integrated emission line is not symmetric around zero velocity (for $\mathrm{H} \beta, \gamma, \delta)$. This also violates the assumptions made for the doppler tomography methods, from which follows $F(v)=F(-v)$ in the orbital average by definition (Eq. (3.13)). To synthesize emission lines according to these two observations, it is neccessary to assume that the object has different "front sides" and "back sides".

\section{Classical Doppler tomograms}

For the two diagnosed lines, $\mathrm{H} \alpha$ and He II $\lambda$ 4686, I calculated Doppler tomograms, using the dopmap v2.3.1 code of Henk Spruit ${ }^{1}$, which uses a maximum entropy method for the reconstruction of the velocity image (Spruit, 1994). The spectra in the range $\phi=0.9 \ldots 1.1$ were omitted in the tomography, since the eclipse may have disturbed the tomogram.

The Doppler map of $\mathrm{H} \alpha$, Fig. 6.20, shows a disturbed disk-like structure: A partial ring of emission is visible at $|v| \approx 300 \ldots 500 \mathrm{~km} \mathrm{~s}^{-1}$. The upper part of that ring $\left(v_{y}>\right.$ $300 \mathrm{~km} \mathrm{~s}^{-1}$ ) is missing. A strongly brightened region is found in the $3^{\text {rd }}$ quadrant of the tomogram. The Doppler tomograms of the other hydrogen lines and the He I $\lambda 7065$ (not shown) reveal the same features, underpinning the earlier statement, that there is a common origin of all neutral emission lines.

A completely different image shows the Doppler map of He II $\lambda 4686$ (Fig. 6.21). As expected from the analyses of the emission line and its orbital behaviour, the tomogram shows a single blob, with the maximum at $\vec{v} \approx(65,-100) \mathrm{km} \mathrm{s}^{-1}$. From the line velocity analysis, a position at $\vec{v} \approx(70,-119) \mathrm{km} \mathrm{s}^{-1}$ would have been expected. The blob extends toward the $-v_{x}$ direction, also a very weak tendency towards the $3^{\text {rd }}$ quadrant cannot completely be ruled out.

These Doppler tomograms show a striking similarity of HS0455 to BH Lyn. In fact, the tomograms of the $\mathrm{H} \beta$ lines from BH Lyn (Hoard and Szkody, 1997) can not be distinguished from $\mathrm{H} \alpha$ the tomograms shown here.

\footnotetext{
${ }^{1}$ At the time of this writing, dopmap can be found at http://www.MPA-Garching.MPG.DE/ henk/
} 


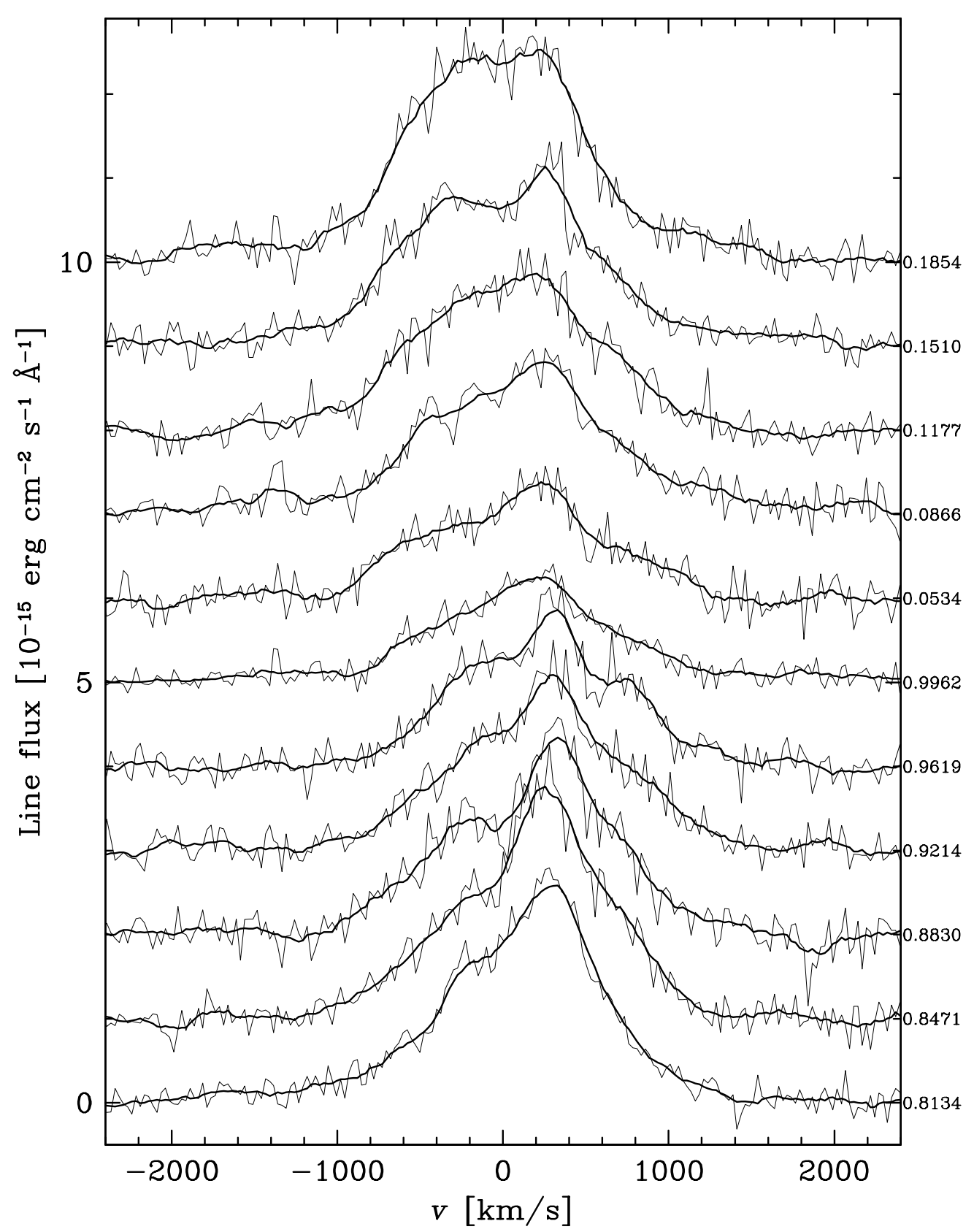

Figure 6.13: The spectra in $\mathrm{H} \alpha$ during the eclipse. The continuum from the disk $F_{\text {disk }} \propto$ $\lambda^{-7 / 3}$ is subtracted, the spectra are offset 1 unit from each other. The number at the right side of the plot denominates the orbital phase. Thin line: original data, thick line: boxcar smoothed data, box width 10 . 


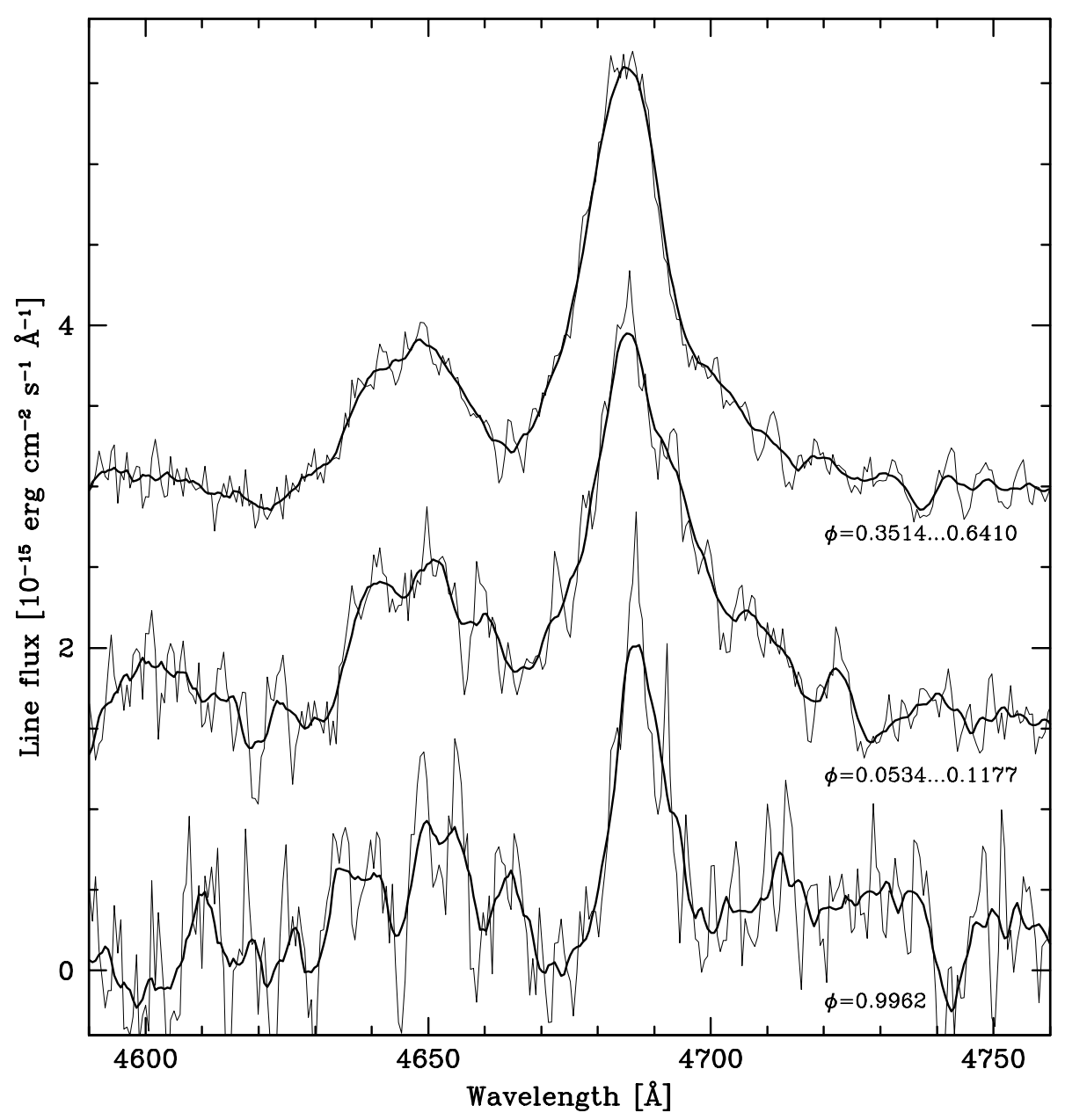

Figure 6.14: The spectrum of He II during the eclipse and outside of the eclipse. The continuum from the disk $F_{\text {disk }} \propto \lambda^{-7 / 3}$ is subtracted, the spectra are offset 1.5 units from each other. Thin line: original data, thick line: smoothed with a boxcar over 10 data points.

From the analysis of the emission line behaviour of HS0455, it has to be concluded, that there are only subtle difference between HS0455 and BH Lyn. BY Lyn has a slightly longer orbital period (224.46 min) than HS0455, and some of the other parameters may also vary within a few percent. Nevertheless, any model that proves to be correct for one of the systems is expected to be valid for the other system as well. 


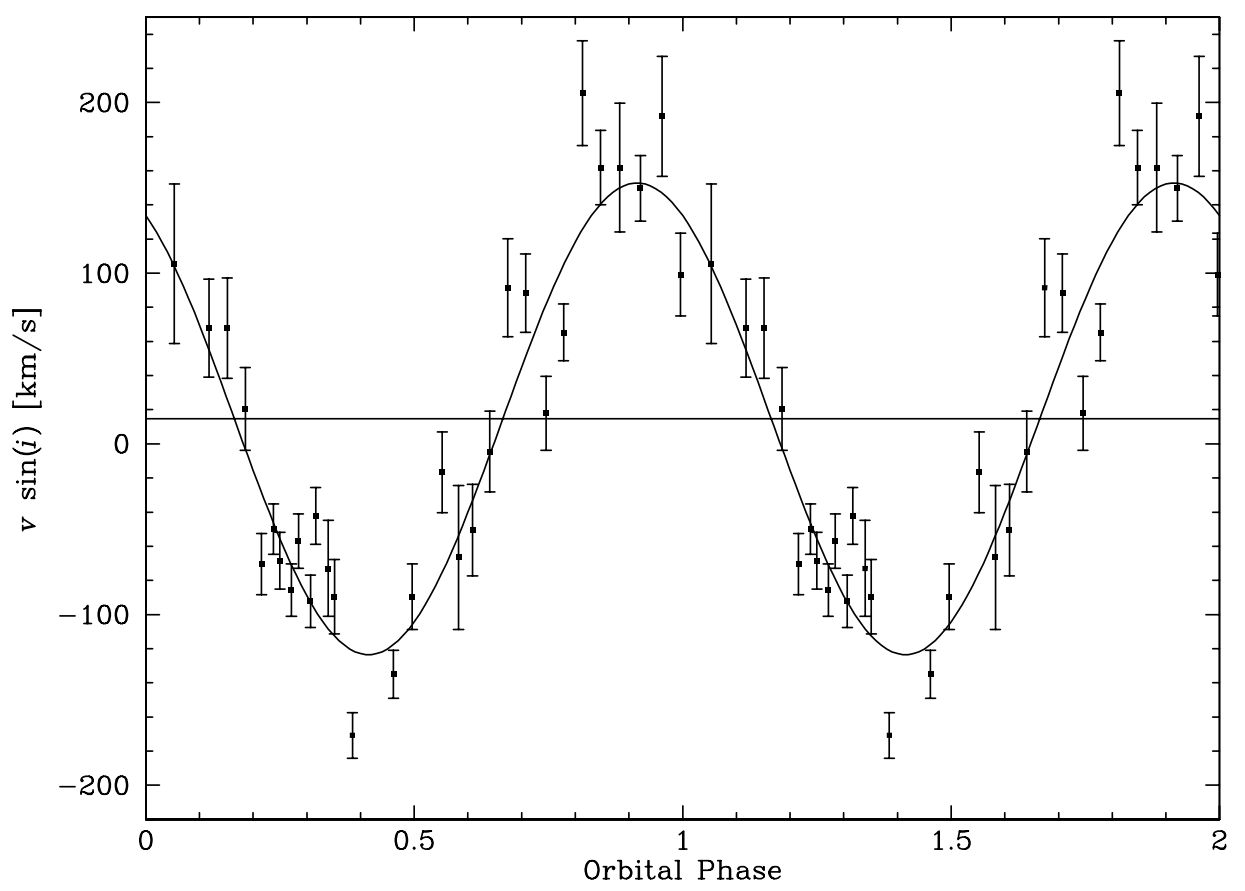

Figure 6.15: Center of the He II $\lambda 4686$ emission line plotted over two orbits for clarity.
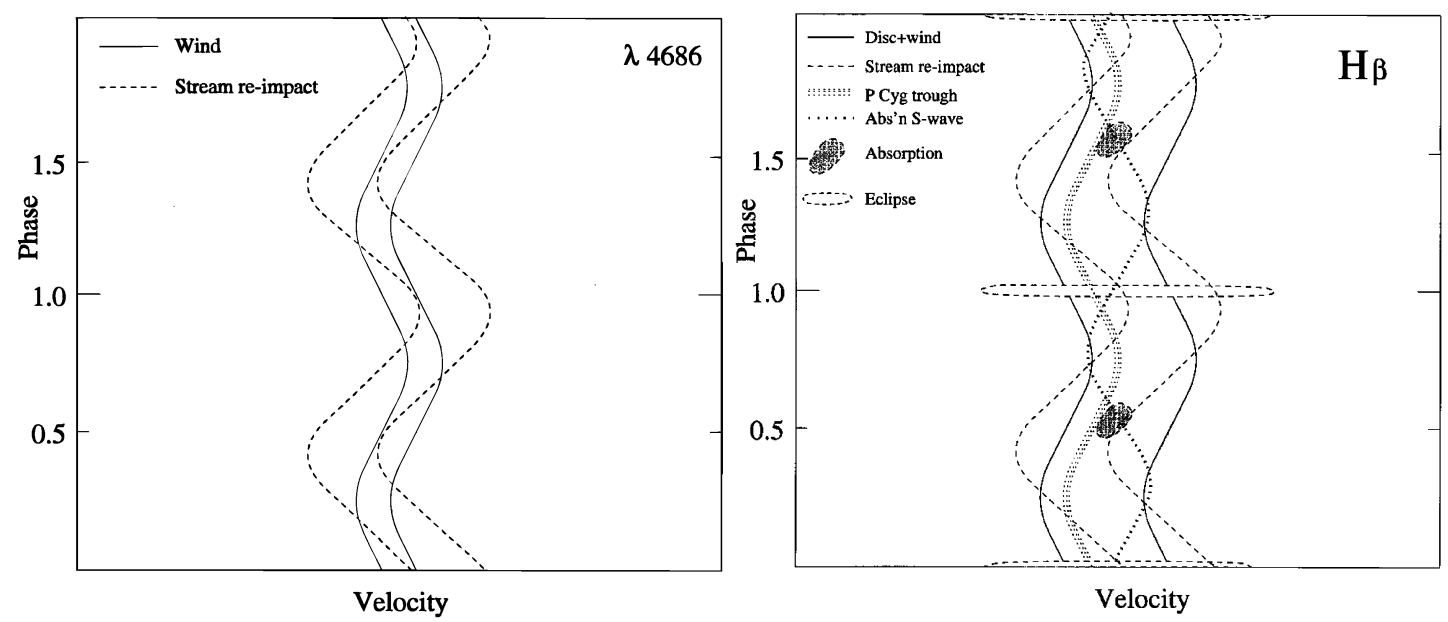

Figure 6.16: Emission line components of a SW Sex system. From Hellier (1996). The right hand figure also applies to the $\mathrm{H} \alpha$ data from HS0455 shown in this work. 


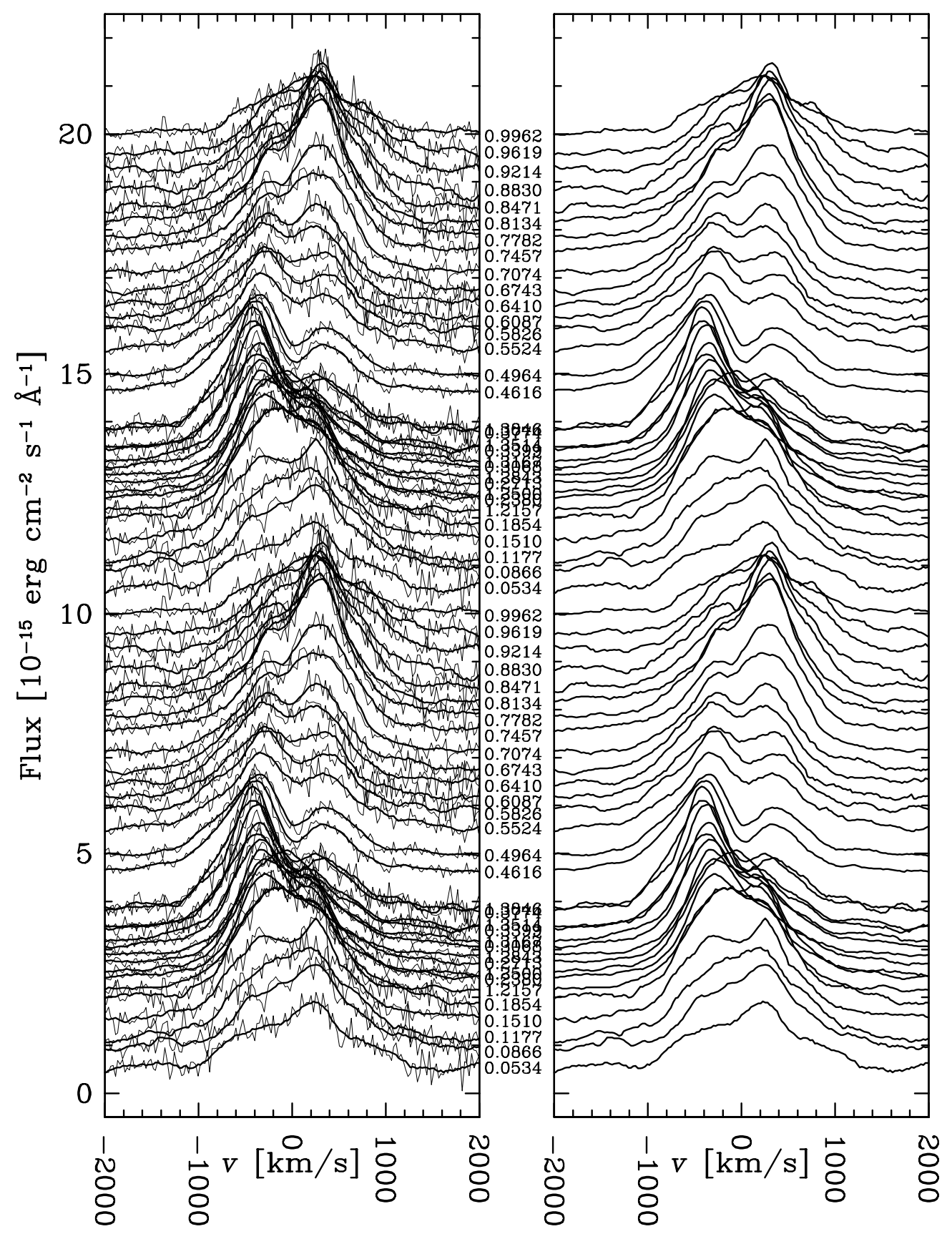

Figure 6.17: The spectra in $\mathrm{H} \alpha$ over the complete orbit. The continuum from the disk $F_{\text {disk }} \propto \lambda^{-7 / 3}$ is subtracted, the spectra are offset from each other according to the orbital phase. The number between the plots denominates the orbital phase. Thin line: original data, thick line: boxcar smoothed data, box width 10. At the right hand panel, only the smoothed data are shown for clarity. 


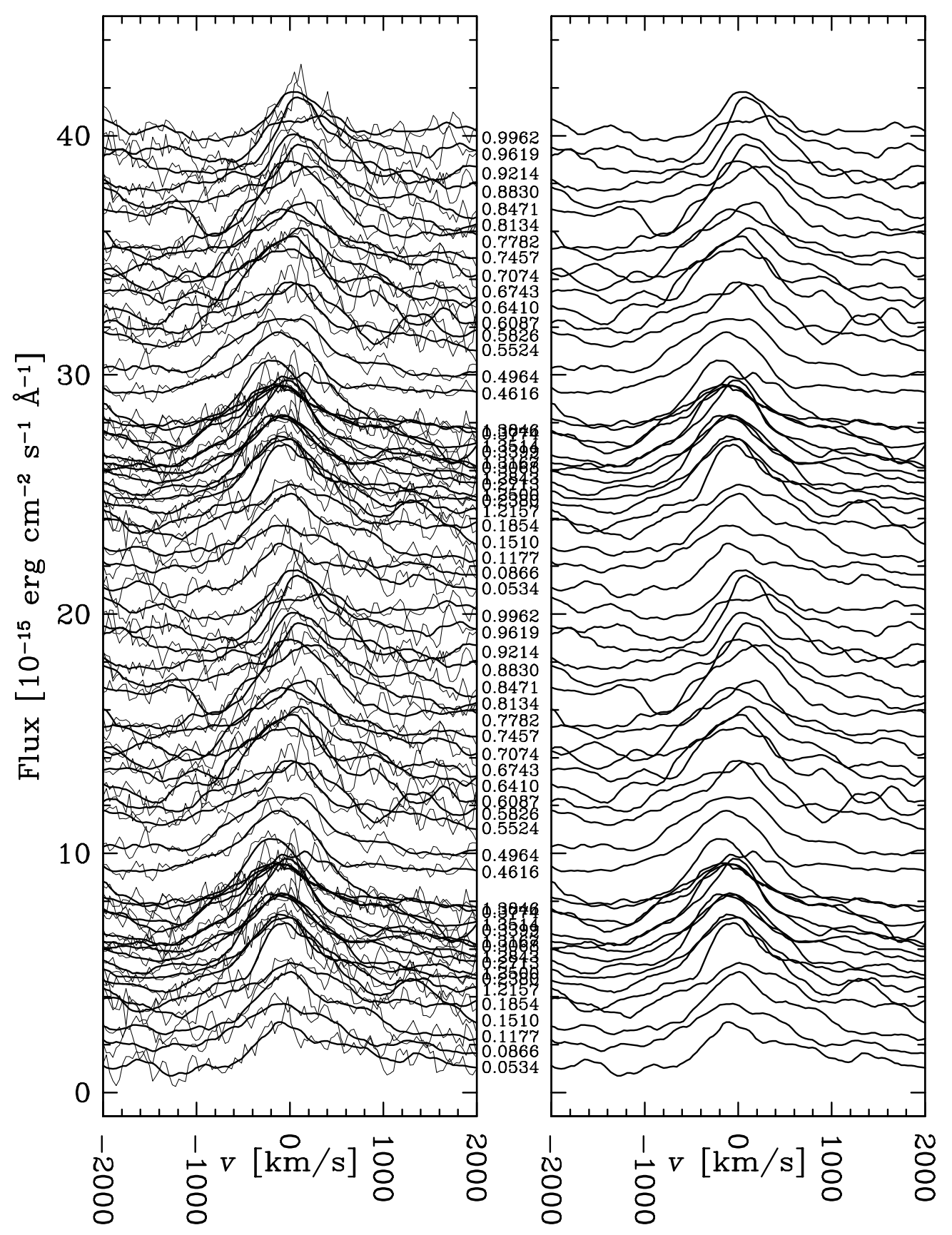

Figure 6.18: The spectra in He II $\lambda 4686$ over the complete orbit. The continuum from the disk $F_{\text {disk }} \propto \lambda^{-7 / 3}$ is subtracted, the spectra are offset from each other according to the orbital phase. The number between the plots denominates the orbital phase. Thin line: original data, thick line: boxcar smoothed data, box width 10. At the right hand panel, only the smoothed data are shown for clarity. 


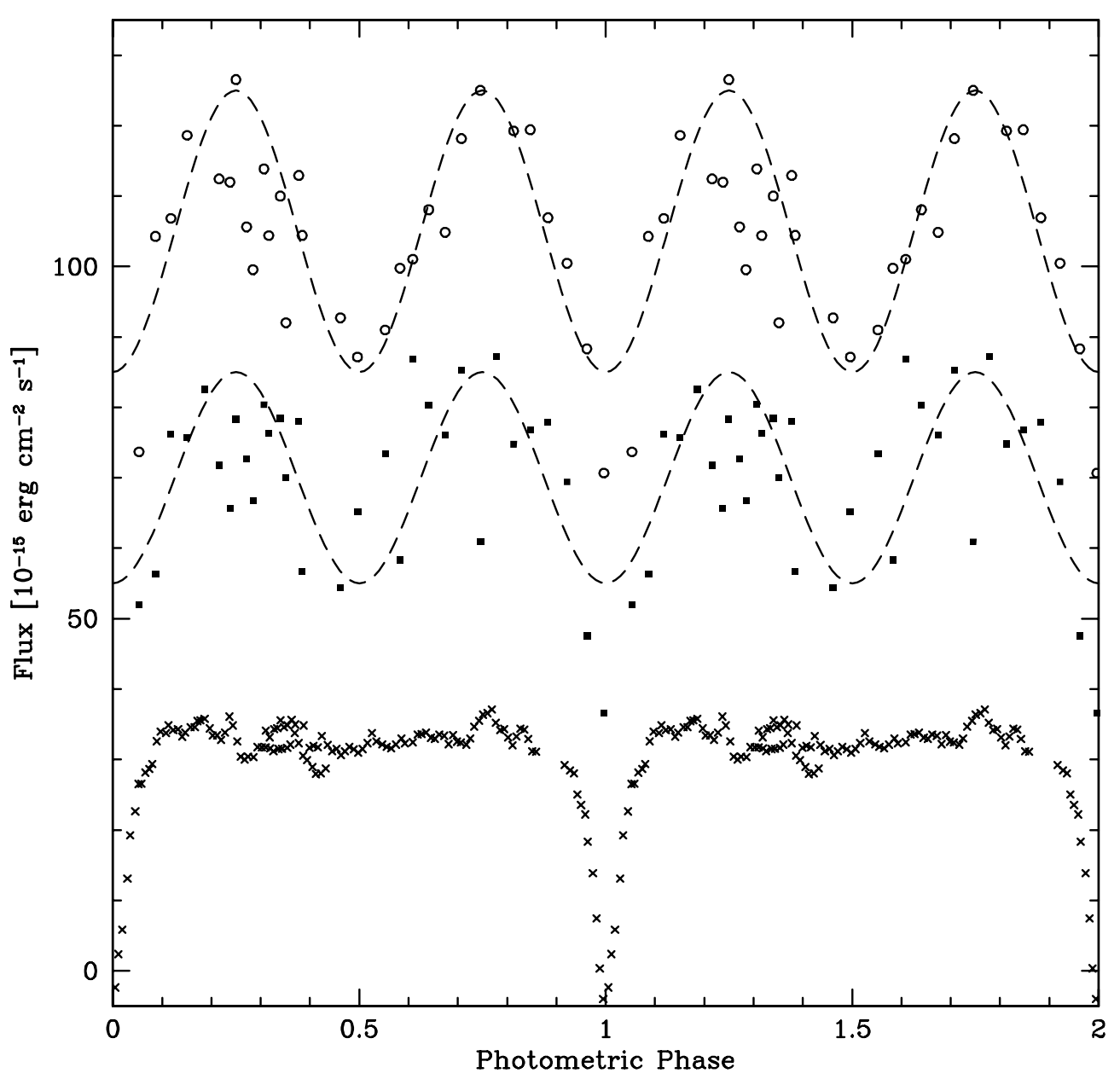

Figure 6.19: Integrated line flux of $\mathrm{H} \alpha$ (open circles), He II $\lambda 4686$ (filled boxes), and the R-band flux (crosses). The $\mathrm{H} \alpha$ flux is offset 10 units to the top, the $\mathrm{R}$ flux is not to scale. Data shown over two orbits. Two best fit sine curves to the emission line light curves are shown as dashed lines. 

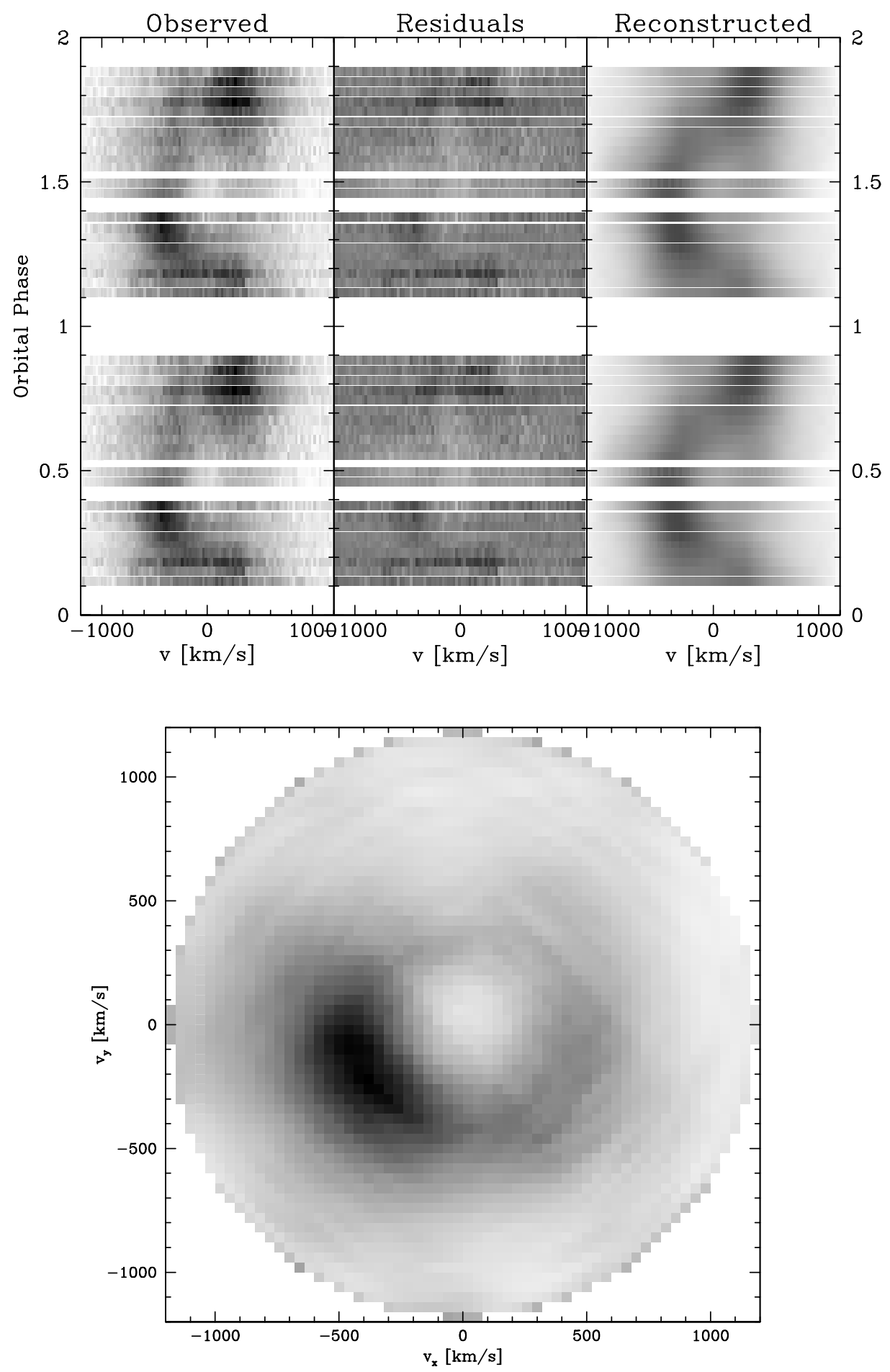

Figure 6.20: Classical Doppler tomogram of HS0455, using the $\mathrm{H} \alpha$ emission line. The observations taken during the eclipse are not used. Top: observed time resolved spectrum (left) and reconstruction (right), bottom: Doppler tomogram 

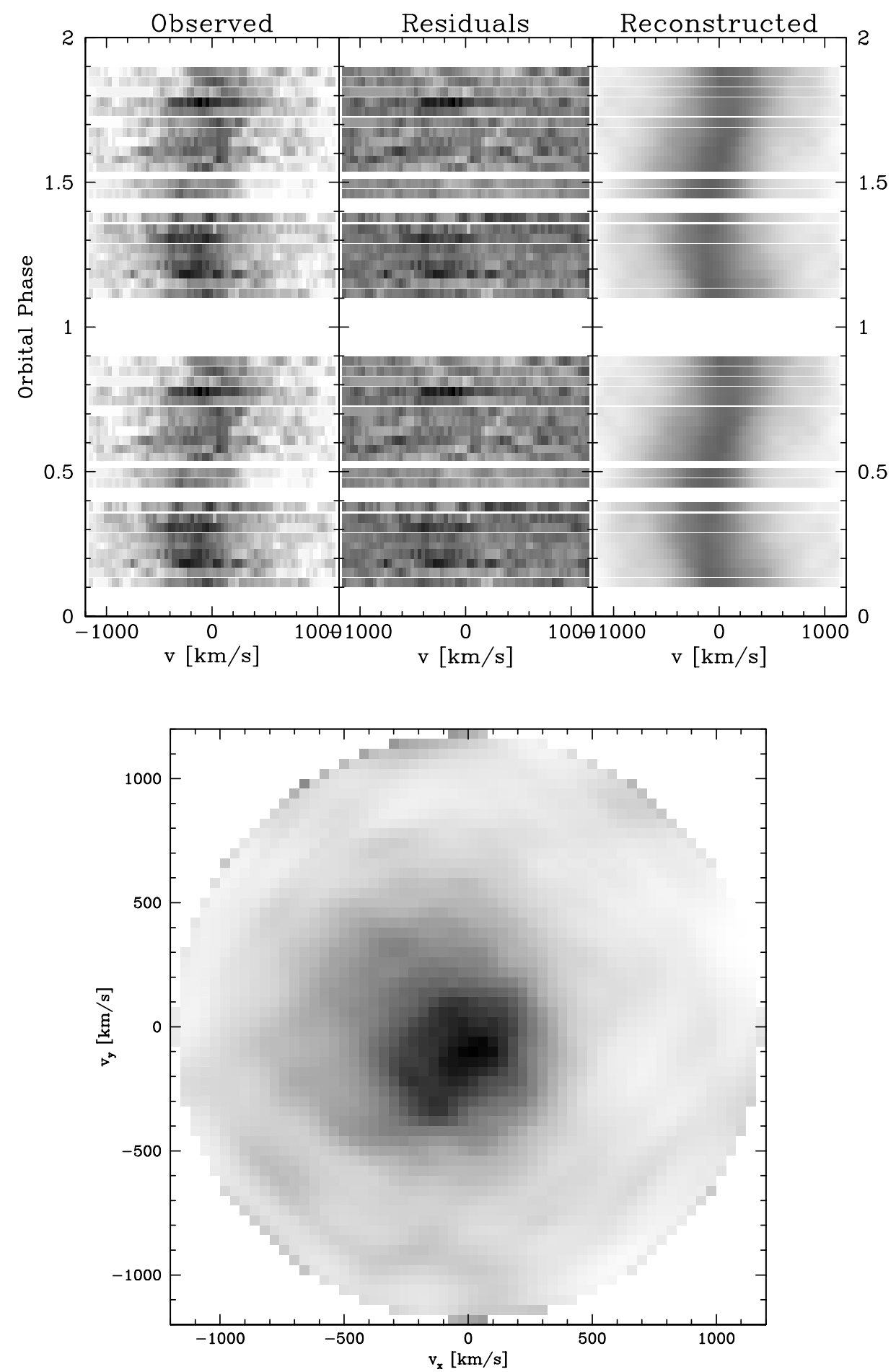

Figure 6.21: Classical Doppler tomogram of HS0455, using the He II emission line. The observations taken during the eclipse are not used. Top: observed time resolved spectrum (left) and reconstruction (right), bottom: Doppler tomogram 


\subsection{Orbital mapping of HS0455+8315}

\subsubsection{The geometrical model of HS0455}

All the previous analyses of the emission lines do not require any assumption about the system geometry. Moreover, many parameters of the geometry can be derived from the properties of the emission lines as described above. To allow a reconstruction of the sources of emission in the accretion structure by means of the new orbital mapping method, it is neccessary to know the geometry and the velocities of the accretion stream and the accretion disk of the system.

The straightforward assumption that the double peaked emission results from a Keplerian accretion disk is inappropriate, as has been discussed for SW Sex systems by many authors (Szkody and Piche, 1990; Thorstensen et al., 1991; Hoard, 1998; Hellier, 2000; Knigge et al., 2000). The lower level of emission around zero velocity has to be interpreted as absorption, which takes places around phase 0.5 (hence, "phase 0.5 absorption"). In the data of HS0455, this also proves to be the correct interpretation, based on reasoning using the kepler velocity of disk:

The orbital period of HS0455 is 214 min, requiring a secondary star with a mass of roughly

$$
M_{2}=0.33 M_{\odot} \quad M_{2}=0.34 M_{\odot} \quad M_{2}=0.28 M_{\odot}
$$

using the period-mass-relations given as the Eqs. (2.10), (2.12), and (2.13) in Sect. 2.3.2. This is also a lower limit of the white dwarf mass to keep the mass ratio $Q=M_{1} / M_{2}>1$ for stable mass transfer. Assuming a mass ratio of $Q=1$ (requiring an inclination of $i=73^{\circ}$ to produce an eclipse width of $\left.\Delta \phi=0.075\right)$ and an accretion disk which extends up $80 \%$ of the distance to $L_{1}$ (Paczynski, 1977), the Kepler velocity of the outer parts

of the disk would be $v_{\mathrm{kep}}=374 \mathrm{~km} / \mathrm{s}$. This is not compatible with the peak distance of the hydrogen line, which is $\approx 600 \mathrm{~km} / \mathrm{s}$ and should be twice the value of the Kepler velocity of the outer disk rim. For other system parameters, the difference between the observed peak distance and the Kepler velocity is even larger.

What is the source of such an absorption feature, how can an accretion disk produce a broad emission line without a double peak, and what are the two hot-spot-like emission maxima which produce the bright arc in the lower left quadrant of the Doppler tomogram? Besides some attempts to explain the observation with disk bulges (Hoard and Szkody, 1997) or magnetic propellers (Horne, 1999) a recent review on SW Sex stars (Hellier, 2000) provides the following interpretation, which can be considered acceptable for HS0455 also:

When the accretion stream hits the disk, its material does not mix instantly with that of the disk. Instead, a significant fraction of approximately $1 / 3$ of the incoming matter is forced above and below the orbital plane ("stream overflow") and hits the 


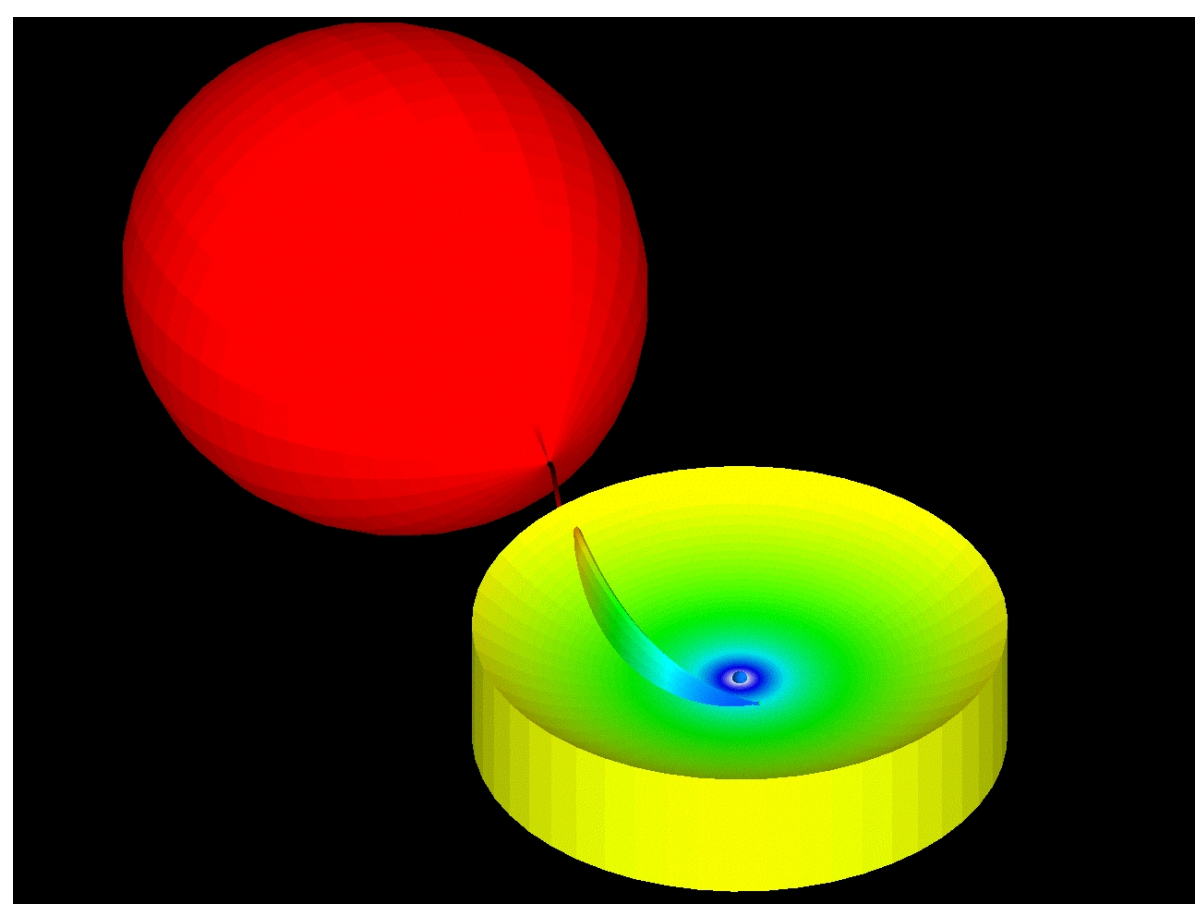

Figure 6.22: Model geometry for HS0455. The colors represent the absolute values of the velocities of the accretion components. The white dwarf and the secondary star are colored arbitrarily. Note that the inclination of HS0455 is close to $80^{\circ}$, so that the WD is hidden behind the rim of the disk.

disk a second time closer to the white dwarf (Armitage and Livio, 1996). Kunze et al. (2001) used smoothed particle hydrodynamcs for their calculations. They find substantial stream overflow for a wide range of system parameters. This stream overflow leads to a structure, following roughly the ballistic trajectory while being extended in $z$-direction (outside the orbital plane). This stream overflow hits the disk again at the circularization radius close to the backside of the white dwarf. The overflowing accretion stream can lead to the observed phase 0.5 absorption. For the emission of the overflowing stream, I follow a suggestion of Hellier and Robinson (1994) by setting the intrinsic $\sigma$ of the line width from the stream to $25 \%$ of the stream velocity. In the actual fit runs, I found that using this broad intrinsic width for the accretion disk also produces significantly better results than setting the intrinsic profile of the disk elements to 0 .

Still, the broad single peak nature of the basic disk emission has to be explained. Knigge et al. (2000) showed that the accretion disk in DW UMa, another member of the SW Sex class of CVs, hides the white dwarf from view during the high state of the system, while the WD is visible in a low state. That leads to the interpretation, that the disk is self-occulting, i.e. has a rim that is high enough that the inner region of the disk and the white dwarf cannot be seen during the high accretion state. Their calculations of the emission lines and continuum light curves from an optically thin 
disk with a dark high rim resemble the flat emission line and the $\mathrm{V}$-shaped continuum eclipse.

The behaviour of the Doppler tomogram results from the superposition of absorption and emission effects of the individual line components. The Doppler image of the accretion disk structure is disrupted by the absorption from the overflowing accretion stream, which reduces the intensity of the ring at velocity coordinates $v_{x} \approx 0 \mathrm{~km} \mathrm{~s}^{-1}, v_{y} \approx 300 \mathrm{~km} \mathrm{~s}^{-1}$. In the lower left quadrant, the emission of the ring from the disk is overlayed by emission from the accretion stream, which produces an apparently brighter region in the disk.

As has already been mentioned in the orbital variation analysis, the overflowing accretion stream allows an explanation for the apparent phase offset of the orbital motion of the emission lines: The observed phase shift is the result of the superposition of the WD centered emission with $\phi_{0}=0$ and the stream emission with $\phi_{0} \approx 0.25$.

Hence, the model which I incorporate for HS0455 is that of an accretion disk which has a sufficiently high rim to hide the white dwarf from view with an added overflowing accretion stream (Fig. 6.22). This overflowing accretion stream is able to absorb any emission which comes from the accretion disk hidden behind. The emission characteristics of the single surface elements of the disk are modeled to be optically thin, following the suggestion from Knigge et al. (2000). The overflowing accretion stream is modeled to be optically thick.

The orbital mapping is done for the $\mathrm{H} \alpha$ line.

\subsubsection{Orbital parameters}

For the determination of the binary parameters I follow the method described by Garnavich et al. (1990). The principle is this: Several equations describe relations between the binary parameters. If the orbital period $P$, the eclipse width at half depth $\Delta \phi_{1 / 2}$, the mass-radius relation of the secondary, and the orbital velocity of the white dwarf $K_{1}$ are known, one can create three relations $R_{1}, R_{2}, R_{3}$, each of which defines a valid set of $(i, q)$, with $q=1 / Q=M_{2} / M_{1}$. So far, the intersection of these relations has been searched graphically by plotting the valid $(i, q)$-sets. I have developed a tool which 
solves all the equations simultanously, using an evolution strategy. The basic equations - given without proof here - are

$$
\begin{aligned}
A=\frac{R_{2}}{R_{\odot}} & =b\left(\frac{M_{2}}{M_{\odot}}\right)^{x} \\
B=\frac{R_{2}}{a} & =\frac{0.49 q^{2 / 3}}{\left(0.6 q^{2 / 3}+\ln \left(1+q^{1 / 3}\right)\right)} \\
C_{B}=\frac{M_{2}}{M_{\odot}} & =\left(73.5 \frac{P^{2}}{\mathrm{~d}^{2}} \frac{q+1}{q} B^{3} b^{-3}\right)^{1 / 3 x-1} \\
D=\frac{M_{2}}{M_{\odot}} & =10^{-7} \frac{P}{\mathrm{~d}}\left(\frac{q+1}{q}\right)^{2}\left(\frac{K_{1} /\left(\mathrm{km} \mathrm{s}^{-1}\right)}{\sin i}\right)^{3} \\
E=\left(\frac{R_{2}}{a}\right)^{2} & =\sin ^{2}\left(\pi \Delta \phi_{1 / 2}\right)+\cos ^{2}\left(\pi \Delta \phi_{1 / 2}\right) \cos ^{2} i \\
C_{E}=\frac{M_{2}}{M_{\odot}} & =\left(73.5 \frac{P^{2}}{\mathrm{~d}^{2}} \frac{q+1}{q} E^{3 / 2} b^{-3}\right)^{1 / 3 x-1} .
\end{aligned}
$$

For the parameters $x$ and $b$, three different sets are used:

$$
\begin{array}{ll}
x=0.88 & b=1.0 \\
x=0.91 & b=1.06 \\
x=0.75 \pm 0.04 & b=0.91 \pm 0.09
\end{array}
$$

the first two taken from Patterson (1984) and Echevarria (1983) for main sequence stars, the third from Smith and Dhillon (1998) for secondaries in CVs and LMXBs.

From the Eqs. 6.23a to 6.23f, the set of equations which has to be fulfilled simultanously can be generated. These equations give the three relations for $(i, q)$ mentioned before:

$$
C_{B}=D \quad C_{E}=D \quad B^{2}=E
$$

The result fulfills the equation

$$
\left|C_{B}-D\right|+\left|C_{E}-D\right|+\left|B^{2}-E\right|=0
$$

and can be found by minimizing the left side of Eq. (6.26) with appropriate fit range boundaries for $i$ and $q$. $A$ is used for later calculations of $a$.

For HS0455, the best fits with the mass-radius relation from Eq. (6.24c) using $\Delta \phi=$ $0.075 \pm 0.002, P=0.148728 \mathrm{~d}, K_{1}=138 \ldots 110 \mathrm{~km} \mathrm{~s}^{-1}$ yield

$$
\begin{aligned}
i & =75.3^{\circ} \ldots 78.2^{\circ} & i & =76.7^{\circ} \\
M_{2} / M_{1} & =0.618 \ldots 0.430 & M_{2} / M_{1} & =0.518 \\
M_{1}+M_{2} & =0.775 M_{\odot} \ldots 0.959 M_{\odot} & M_{1}+M_{2} & =0.856 M_{\odot} \\
M_{1} & =0.479 M_{\odot} \ldots 0.670 M_{\odot} & M_{1} & =0.564 M_{\odot} \\
M_{2} & =0.296 M_{\odot} \ldots 0.288 M_{\odot} & M_{2} & =0.292 M_{\odot}
\end{aligned}
$$


The latter results $\left(i=76.7^{\circ}\right)$ are used for the following analysis. A comment on the range used for $K_{1}$ : Since $K$ as presented in Eq. (6.21) for the He II $\lambda$ 4686-line consists of components which are centered around the white dwarf as well as higher velocity components from the accretion stream, the value found is an upper limit to $K_{1}$. The real $K_{1}$ is certainly in the range indicated above, for the "best values" I used $K_{1}=$ $128 \mathrm{~km} \mathrm{~s}^{-1}$.

\subsubsection{Fit results}

The fit runs using the orbital mapping method on the data of HS0455 with the model described above produce fits which are not perfect but still of some diagnostic value. This is a result of the complicated nature of the emission lines from SW Sex stars, which is sketched in Fig. 6.16.

By using the orbital parameters derived in the above section, it is impossible to create an emission line profile with the width observed. It is neccessary to assume the disk to rotate with a sub-Keplerian velocity in its outer regions $\left(v \approx 0.7 v_{\text {kep }}\right)$.

To indicate which parts of the spectrum are affected by the overflowing stream, I also calculated a map of a system without the stream component (Fig. 6.23). Although the overall impression of this fit is not much worse than that of the fits with an overflowing stream (Figs. 6.24 and 6.25), some details visible especially in the residuals emphasize the contribution of an overflowing stream. First, high velocity emission in the original data is visible at $\phi=0.4 \ldots 0.7, v=-600 \ldots-1000 \mathrm{~km} \mathrm{~s}^{-1}$ and at $\phi=0.8 \ldots 1.0, v=+600 \ldots+1000 \mathrm{~km} \mathrm{~s}^{-1}$ as black features. Second, the increased intensity at $\phi=0.8 \ldots 0.9, v=+400 \mathrm{~km} \mathrm{~s}^{-1}$ is not reconstructed sufficiently, again visible dark in the residuals. Both of these structures are attributed to the overflowing stream.

The best fit results for the model of a disk with an overflowing stream were achieved by fitting a radially symmetric intensity behaviour of the disk as described on p. 67 . Additionally, the accretion stream was parametrized. In one attempt, the weighting of the accretion stream against the disk was adjusted using the parameter $\gamma_{1}$ :

$$
\text { case I: } \quad I_{i, \text { stream }}=I_{i}^{\prime} \cdot \gamma_{1}
$$

In order to take into account possible larger changes of the stream intensity like they would be required by a re-impact model, another fit using five parameters for the stream was executed:

$$
\text { case II: } \quad I_{i, \text { stream }}=I_{i}^{\prime} \cdot \max \left[\left(\gamma_{2}+\gamma_{3}\left(r_{i} / a-\gamma_{4}\right)+\gamma_{5}\left(r_{i} / a-\gamma_{6}\right)^{2}\right), 0\right]
$$

with $r_{i}$ the distance from the $i$-th element to the WD. This simulates a quadratic approximation to the intensity profile of the accretion stream. The best fit results are shown in Figs. 6.24 and 6.25. Note that the inclination of the maps in these figures is 

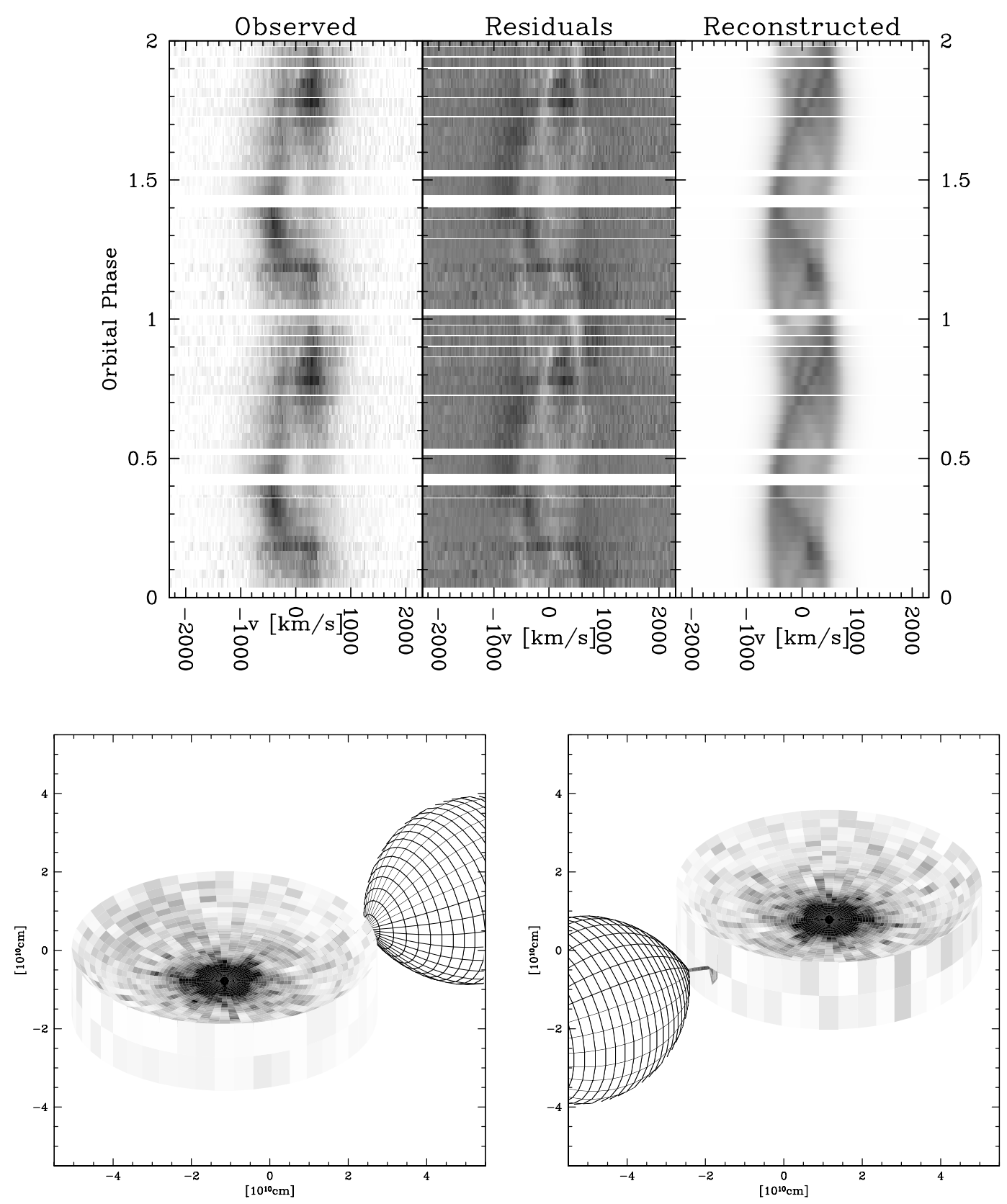

Figure 6.23: HS0455 fit result. Top: Trailed spectrum of He II $\lambda$ 4686, bottom left: map as seen from $\phi=0.35$ with $i=60^{\circ}$, bottom right: map with $\phi=0.85$. No overflowing stream included.

set to a lower value $\left(i=60^{\circ}\right)$ than was used for the reconstructions $\left(i=76.7^{\circ}\right)$ to allow a better view on the accretion disk and the stream. Additionally, Fig. 6.26 shows the intensity profiles of the accretion disk and the accretion stream. 

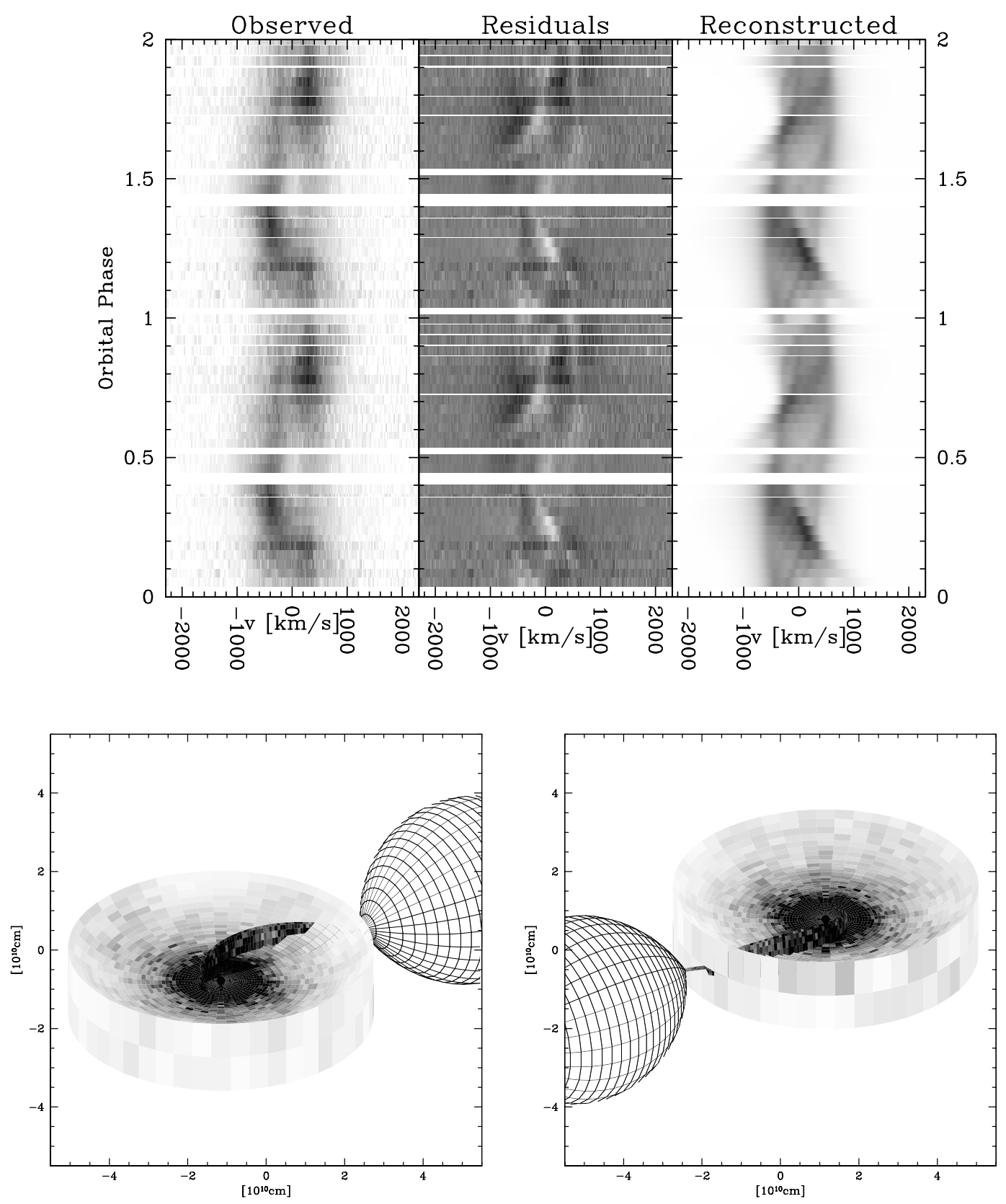

Figure 6.24: HS0455 fit result. Top: Trailed spectrum of He II $\lambda$ 4686, bottom left: map as seen from $\phi=0.35$ with $i=60^{\circ}$, bottom right: map with $\phi=0.85$. Stream parametrized with a constant factor, Eq. (6.28).

Both reconstructions lead to a similar radial intensity of the disk, having

$$
\begin{gathered}
I_{\text {caseI }} \propto r^{-2.4} \\
I_{\text {caseII }} \propto r^{-2.1} .
\end{gathered}
$$



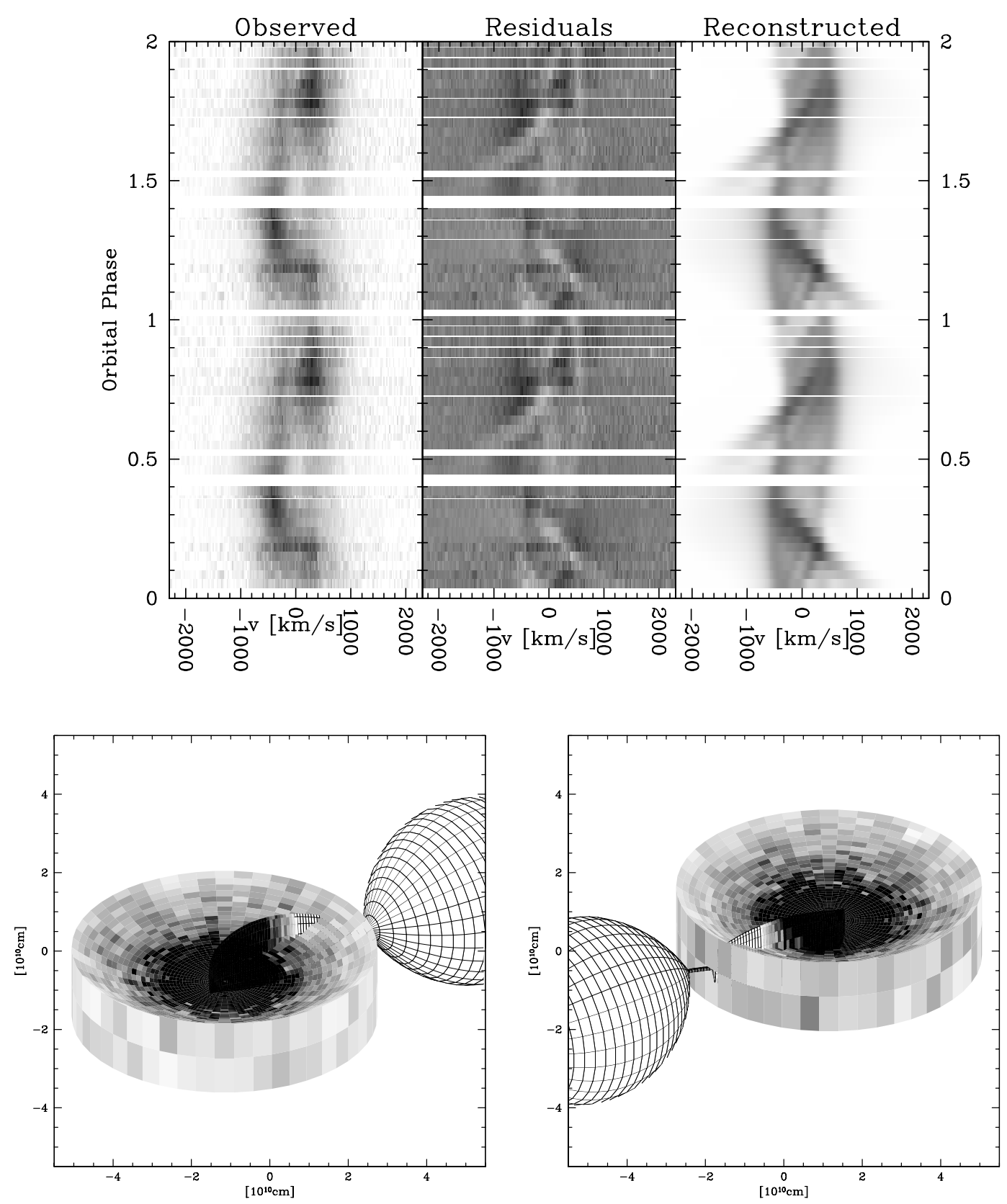

Figure 6.25: HS0455 fit result. Top: Trailed spectrum of He II $\lambda$ 4686, bottom left: map as seen from $\phi=0.35$ with $i=60^{\circ}$, bottom right: map with $\phi=0.85$. Stream parametrized with a $2^{\text {nd }}$ grade polynomial, Eq. (6.29).

However, these two numbers must not be taken literally as the real intensity structure. Test runs with different disk height profiles which lead to a flatter rim or a less convex disk produced significantly different radial profiles with slopes down to $I \propto r^{-1.2}$.

Looking at the inner part of the emission line, both cases lead to a good agreement in the phase range $\phi=0 \ldots 0.4$. They both create too flat a phase- 0.5 absorption. The small 

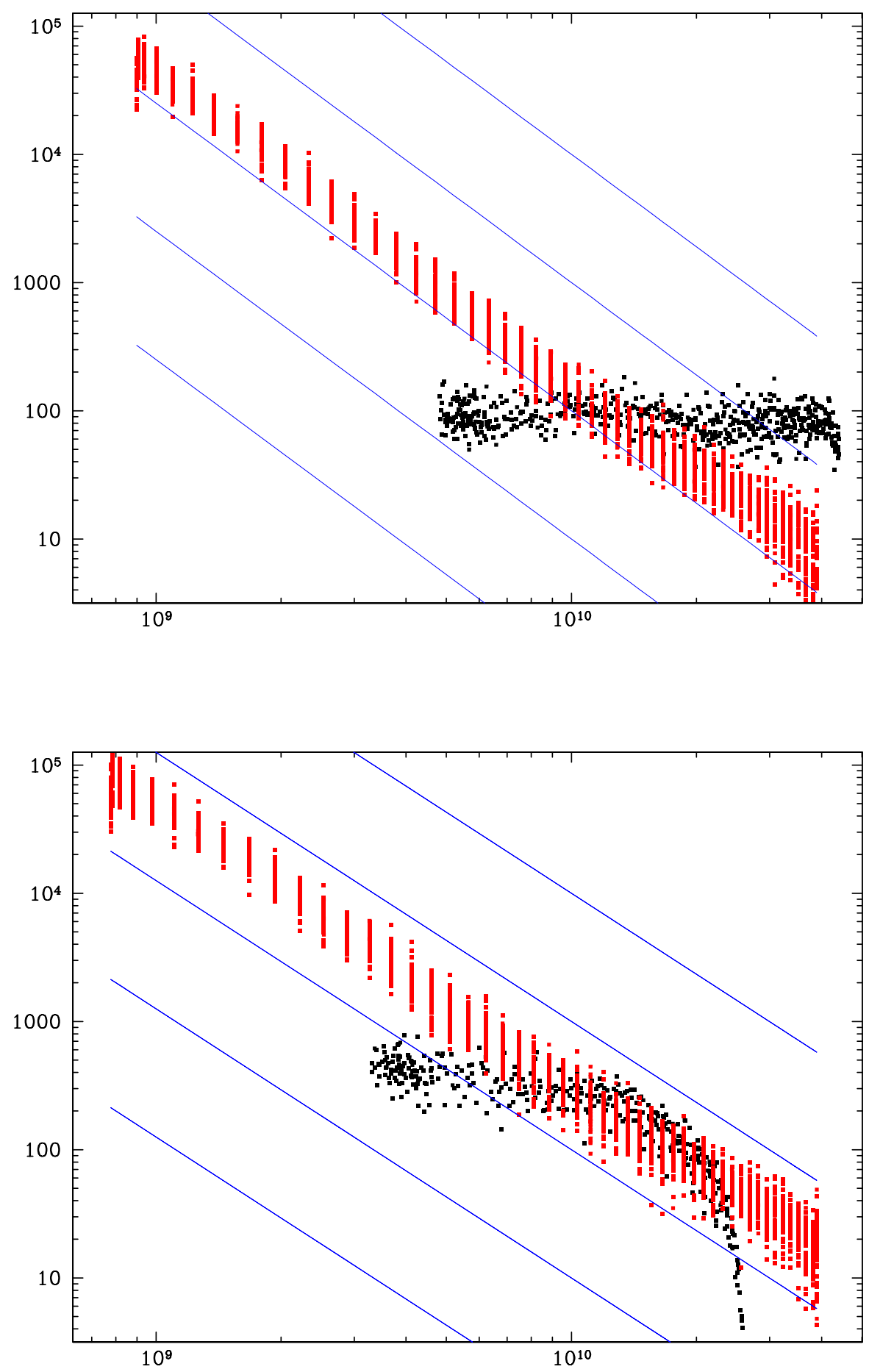

Figure 6.26: HS0455 fit result. Intensity profiles of the disk (red) and the accretion stream (black). Top: stream parametrized with a constant factor, Eq. (6.28). Bottom: stream parametrized with a $2^{\text {nd }}$ grade polynomial, Eq. (6.29). Lines indicate the average intensity gradient of the disk. 
absorption feature so far uncommented which is seen at $v=-100 \mathrm{~km} \mathrm{~s}^{-1}, \phi=0.7 \ldots 0.9$ can be attributed to an expanding shell around the binary star, producing a $\mathrm{P}$ Cyg type absorption (phase 0.8 absorption) which may also account for some of the phase0.5 absorption. Since no P Cyg profile is included in the geometrical model, the exact reconstruction of both the phase 0.8 and the phase 0.5 absorption had to fail.

In the comparison with Fig. 6.23, the increase in quality of the model description compared to a model without stream overflow is apparent: Although not perfectly reconstructed, only the overflow models are able to produce intensity at the highvelocity component. The discrepancy between the modeled and the observed highvelocity emission is found at $\phi=0.7 \ldots 0.8$ and can be attributed to the simplicity of the stream overflow model. The real overflowing stream may have a different velocity structure than the assumed ballistic trajectory as well as a slightly different location in space.

The case II study reveals a remarkable result: At the beginning of the iterative fit process, the polynomial modeling the stream intensity is set to $I=\operatorname{const}(r)$, leaving all the decisions about if and where the stream has increased brightness to the fit algorithm. In several fit runs which are neccessary to prove the stability of the result reached with an evolution strategy, the result always was as shown in Fig. 6.26, bottom: The polynomial is adjusted in such a way that the maximum of the stream emission is close to the white dwarf. Looking at the spectra, especially comparing the residuals of the fit runs of case I and case II, one finds the spectral signature of that result: That is the high velocity component, which is only weak in the reconstruction of case I, leading to a flux depletion seen as a dark region at the residuals in Fig. 6.24 at $v=-600 \cdots-$ $1000 \mathrm{~km} \mathrm{~s}^{-1}, \phi=0.4 \ldots 0.7$ and - not as strong - at $v=+600 \cdots+1000 \mathrm{~km} \mathrm{~s}^{-1}$, $\phi=0.8 \ldots 1.0$. These regions are significantly better reproduced in case II (Fig. 6.25). Also significantly better reproduced in case II than in case I is the bright region at $\phi=0.8 \ldots 0.9, v=+400 \mathrm{~km} \mathrm{~s}^{-1}$. Reconstructions without an overflowing accretion stream naturally fail to account for the high velocity component, as described above. To make certain that the brightness variation of the overflowing stream is no artificial effect of the method, I made test calculations in which I tried to fit an overflowing stream to synthetic data in which no overflowing stream was present. The algorithm accounted for that by reducing the intensity of the overflowing stream to a very low value, rendering it invisible in the spectrum. Hence, the existence of an overflowing stream which is bright at the region where it re-impacts to the disk is supported by this analysis. 


\subsection{Orbital mapping of V436 Centauri}

As a second and more straightforward application of the orbital method, I present data of the SU UMa star V436 Cen.

V436 Cen has an orbital period of $90.001 \mathrm{~min}$. The Balmer lines are double-peaked, as can be clearly seen in the integrated spectrum around $\mathrm{H} \beta$ and $\mathrm{H} \gamma$ (Fig. 6.27). In earlier works, the inclination of V436 Cen has been estimated to be $65^{\circ} \pm 5^{\circ}$. V436 Cen is an X-Ray source and shows QPOs. A detailed spectroscopic analysis of V436 Cen has been carried out by Gilliland (1982), who estimated the inclination to be $i \lesssim 50^{\circ}$ and $M_{1} \gtrsim 0.8 M_{\odot}$. UV spectroscopy and subsequent fitting of model spectra for the disk and the white dwarf yielded $M_{1}=0.8 M_{\odot}$ and $i=75^{\circ}$ (Nadalin and Sion, 2001). The high inclination of the later paper is hard to believe, since the systems shows no sign of even an partial eclipse.

V436 Cen was observed at the VLT UT 2 (Kueyen) telescope by Klaus Reinsch on May 16, 2001 when the system was in quiescence. The data used in the following analysis was taken with the FORS2 spectrograph in the HIT-5 mode with the G1400V grism. 6 frames with an exposure time of $627 \mathrm{~s}$ each were taken with a 1 " slit, 2 with a 0.5 " slit. Each frame contains 2048 rows holding a spectrum exposed for $0.307 \mathrm{~s}$. This data of high time resolution was rebinned to intervals of of $\Delta \phi=0.0171$, i.e., 92.1 s. That led to 48 spectra distributed unevenly around the binary orbit. The wavelength calibration was done using the theoretical dispersion of $0.5 \AA / p x$ for the instrument and the center of the emission line as the wavelength reference. This is sufficiently exact, since only very small wavelength regions around the emission lines are used for the OMA method. No systemic velocity could be derived by this reduction method.

No simultanous photometry for the observed data is available. Hence, the intensity calibration is somewhat uncertain. Previous photometric analysis of V436 Cen in quiescence (Bailey, 1979) showed no orbital variation. Therefore, I adjusted the intensities of the single spectra in such a way that a 10 pixel wide boxcar smoothed version of the spectra has its maximum at unity. An attempt to keep the integrated line flux constant showed slightly different and less satisfying results.

\subsubsection{System geometry}

Since the published ephemeris for V436 Cen is too old to allow a precise phasing of the data set, I carried out an orbital-motion analysis for the present data. Fitting the width and the sinusoidal variation of the center of a Gaussian to the observed line wings allowed to determine the orbital phase, with $\phi=0$ at inferior conjuction of the 
Table 6.5: Model geometry for V436 Cen.

\begin{tabular}{lccc}
\hline \hline Parameter & Set 1 & Set 2 & Set 3 \\
\hline$M_{2} / M_{\odot}$ & 0.14 & 0.19 & 0.072 \\
$M_{1} / M_{2}$ & 2.2 & 3.5 & 3.9 \\
$i /^{\circ}$ & 45 & 31 & 49 \\
$r_{\text {disk }} / a$ & 0.5 & 0.46 & 0.47 \\
\hline \hline
\end{tabular}

secondary star. This analysis yields a $K_{1}$ significantly different from that of Gilliland (1982):

$$
\begin{array}{ll}
K_{1}=59 \pm 8 \mathrm{~km} \mathrm{~s}^{-1} & \text { Gilliland (1982), } \mathrm{H} \beta, \gamma, \delta \\
K_{1}=88 \pm 5 \mathrm{~km} \mathrm{~s}^{-1} & \mathrm{H} \beta \\
K_{1}=88 \pm 2 \mathrm{~km} \mathrm{~s}^{-1} & \mathrm{H} \gamma
\end{array}
$$

The $\mathrm{H} \beta$ data are slightly more uncertain than the $\mathrm{H} \gamma$ data, since every sixth data set of $\mathrm{H} \beta$ suffers from a set of some uncorrected bad pixels around $v \approx-1400 \mathrm{~km} \mathrm{~s}^{-1}$.

I calculated OMA fits for three different model geometries, which all gave similarly good fits and the same radial dependencies (see below). The geometrical parameters are listed in Tab. 6.5. The first data set is generated using the high value of $K_{1}$ found in my analysis, while the data sets 2 and 3 reproduce the low $K_{1}$ (Gilliland, 1982).

\subsubsection{Doppler tomograms}

From the rebinned data set, Doppler tomograms for the $\mathrm{H} \beta$ and the $\mathrm{H} \gamma$ line were calculated (Fig. 6.28). Both Doppler images are very similar. They show a slightly disturbed accretion disk. In the $\mathrm{H} \gamma$ tomogram, one can find that the distortion of the disk is limited to the inner areas in velocity space, indicating that the outer (hence slower) regions of the disk may be affected by the observed distortion, while the inner regions (visible as the constant velocity space intensity at around $v \approx 1000 \mathrm{~km} \mathrm{~s}^{-1}$ ) remain undisturbed.

The Doppler maps were generated by Bettina Hoffmann using the Fourier-filtered backprojection method. The speckles of the images are a direct result of this method, since no smoothing as in the MEM approach is applied.

\subsubsection{Orbital mapping}

The Doppler tomograms and the trailed spectra show no strong assymetric components. No hot spot, stream overflow, or other features are expected in the system. Hence, the 


\section{Excursion: Geometry of a non-eclipsing disk system}

If the double peak structure of a Keplerian disk in a non-eclipsing system is visibile, one can measure (a) the Kepler velocity at the outer disk rim $v_{\text {out }}$ : this is given by half the distance between the maxima of the peaks; (b) the Kepler velocity at the inner disk rim $v_{\text {in }}$ : this is the velocity where the intensity of the observed spectral line is indistuinguishable from the continuum.

By using the mass-radius relation from Eq. (2.12) and the relation between $K_{1}$, $q$, and $i$ given by Eq. (6.23d), one gets a valid set of $(i, q)$-values. From these, a single valid solution can be obtained by assuming that the maximum inner velocity is reached at a disk radius identical to the WD radius: The Kepler velocity at this radius is given by Eq. (2.8): $v_{\text {in }}=\sqrt{G M_{1} / r_{\mathrm{WD}}}$. The radius of the white dwarf is approximated by (Nauenberg, 1972):

$$
r_{\mathrm{WD}}=0.779 \times 10^{9} \mathrm{~cm} \cdot \sqrt{\left(\frac{M_{1}}{1.44 M_{\odot}}\right)^{-\frac{2}{3}}-\left(\frac{M_{1}}{1.44 M_{\odot}}\right)^{\frac{2}{3}}} .
$$

For the solution found by combining the mass-radius relation of the secondary,its mass orbital-velocity period relation, the Kepler-velocity WD radius and the WD-mass WD-radius relation, I calculate the disk size $r_{\text {disk }}$ needed to produce the observed outer rim velocity, again using the Kepler velocity formula. The maximum size of the disk is determined by the tidal disruption radius (Paczynski, 1977)

$$
r_{\max } \approx \frac{0.60 a}{1+q}
$$

and should not be exceeded by $r_{\text {disk }}$ in the set of solutions to the orbital parameter equations.

geometry which I assume for the orbital mapping of V436 Cen is that of a geometrically thin disk with negligible flaring or convexity. For the assumed geometry "1" with an inclination of $i=45^{\circ}$ and a WD mass of $M_{1}=0.31 M_{\odot}$, the disk has to extend to $0.5 a$ which is $0.85 R_{L_{1}}$, slightly larger than the maximum size of a disk limited by the tidal disruption radius. The disks for the sets " 2 " and " 3 " have radii smaller than the disruption radius. The reconstructions are carried out fitting a radial intensity component to the disk (Eq. (6.15)). The emission characteristics of the disk elements was modeled both as optically thin and optically thick. First attempts in which only the instrumental width of the emission lines is taken into account lead to an insufficient fit quality. Hence I included an intrinsic line width of the surface elements of

$$
\sigma_{\mathrm{intr}, i}=0.25 \cdot\left|v_{i}\right|
$$



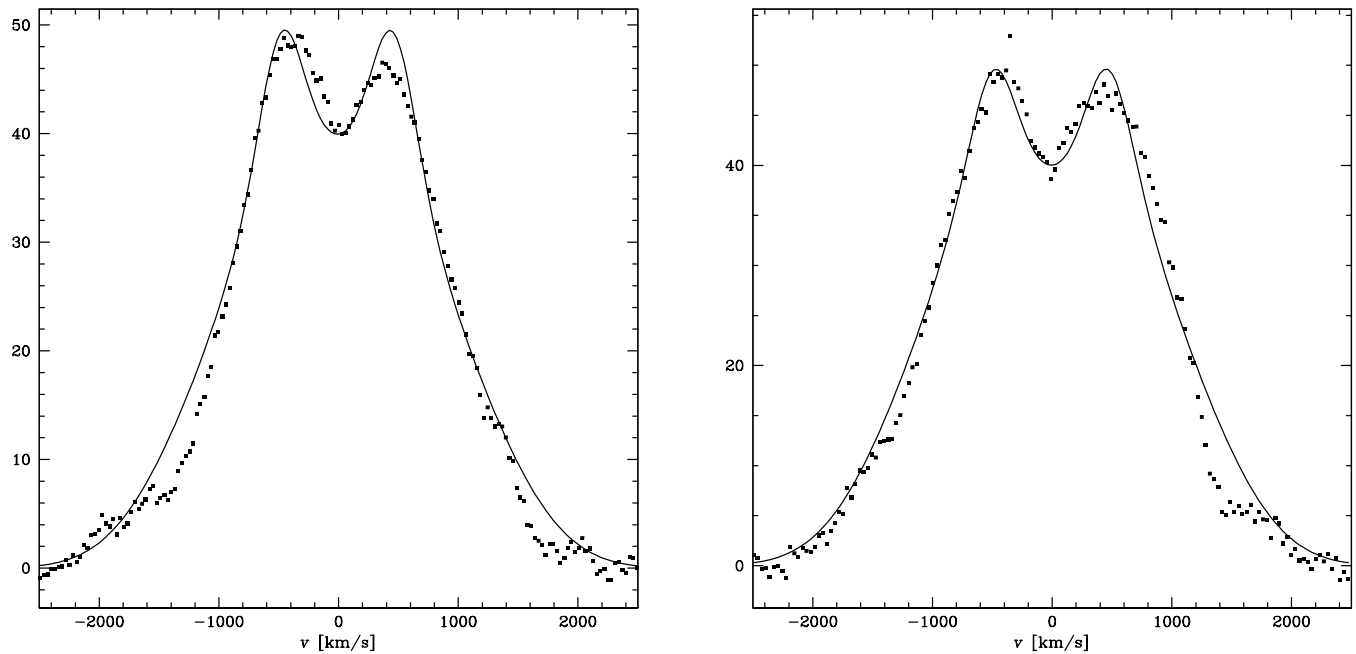

Figure 6.27: Integrated emission lines of V436 Cen. Left: $\mathrm{H} \beta$, right: $\mathrm{H} \gamma$. The observed data are plotted as points, the spectrum of the reconstruction is shown as a line. Not corrected of orbital motion.
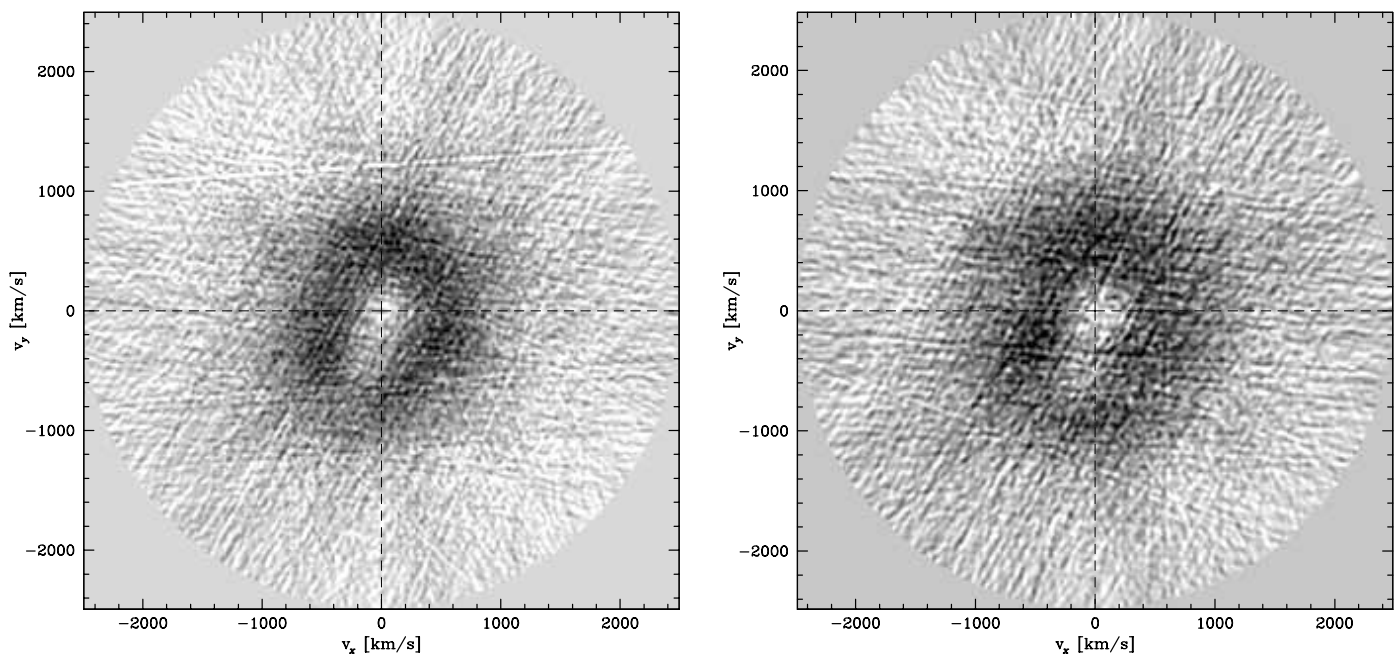

Figure 6.28: Doppler tomograms of V436 Cen. Left: $\mathrm{H} \beta$, right: $\mathrm{H} \gamma$. Tomograms calculated by Bettina Hoffman with her Fourier-filtered back-projection code.

which increased the fit quality especially in the wings of the emission line.

The results of the fit are shown in Figs. $6.29(\mathrm{H} \beta)$ and $6.30(\mathrm{H} \gamma)$. I show the results for geometry 1 and optically thick emission, only. The other geometries do not show significantly different results. 

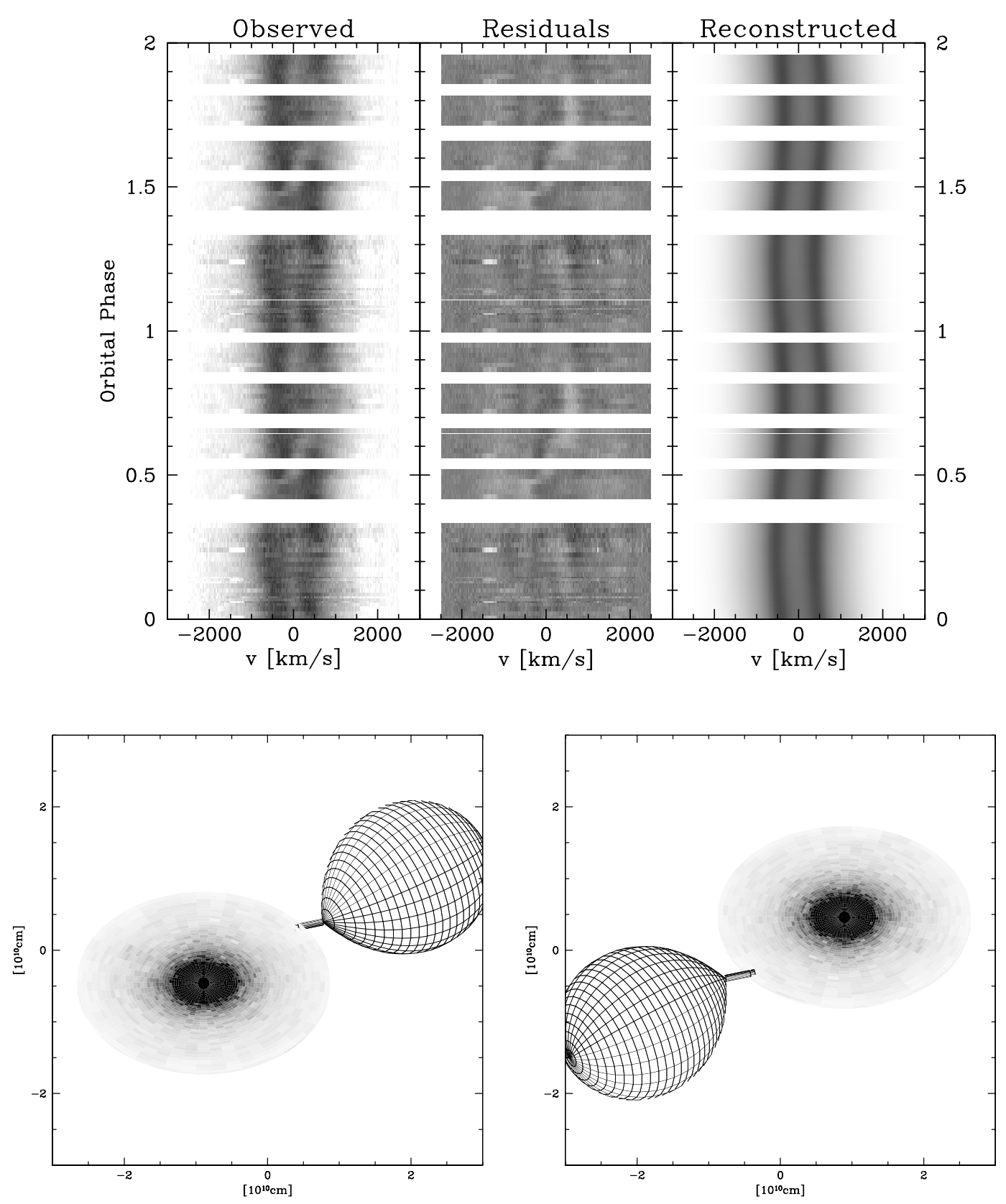

Figure 6.29: V436 Cen: $\operatorname{map}(\phi=0.35, \phi=0.85)$, spectrum, and reconstruction. $\mathrm{H} \beta$

No features apart from the radial symmetric component are detected by the orbital mapping method. The radial dependency was found to be

$$
\begin{array}{ll}
I \propto r^{-2.3} & \mathrm{H} \beta \\
I \propto r^{-2.5} & \mathrm{H} \gamma .
\end{array}
$$



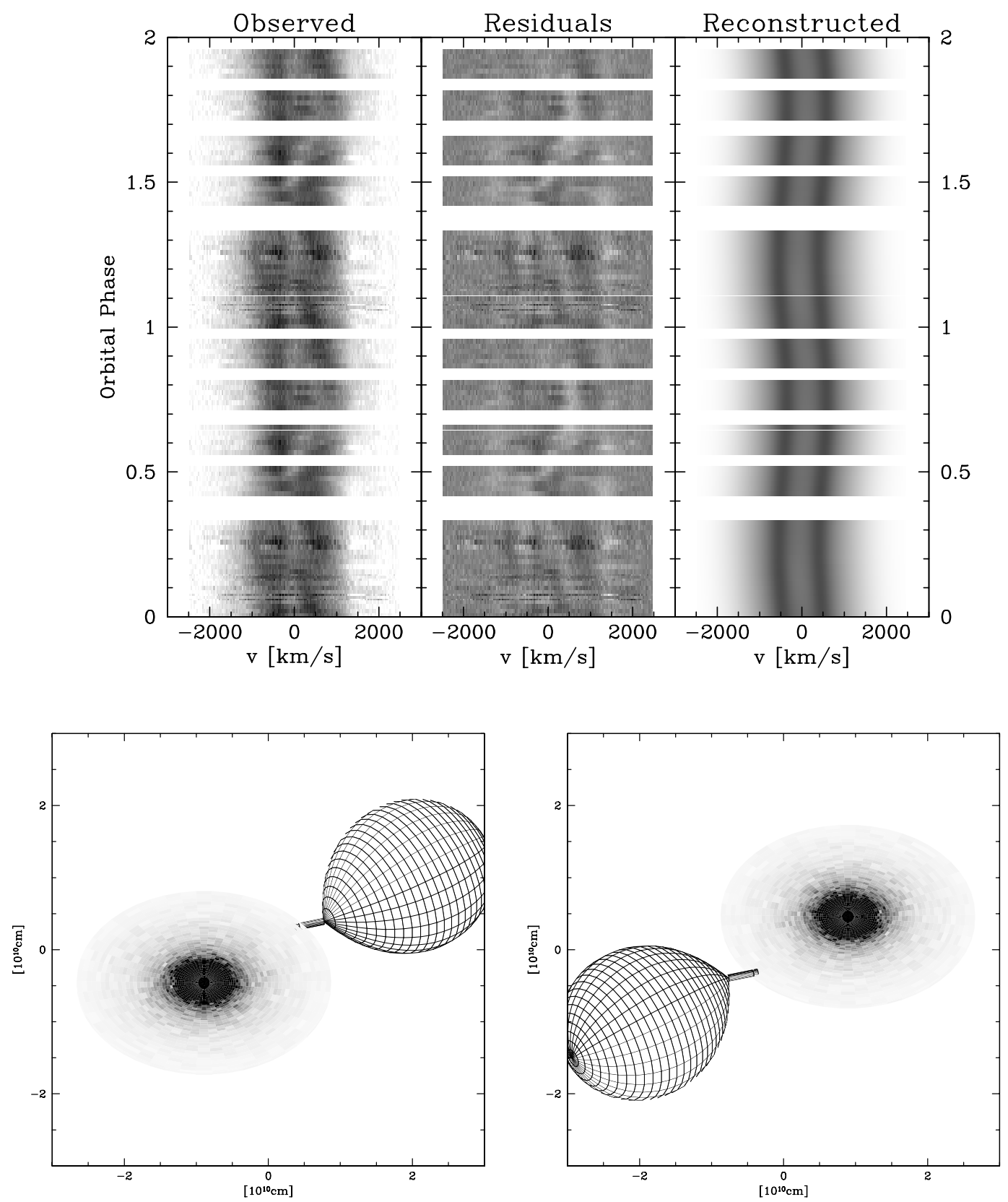

Figure 6.30: V436 Cen: map $(\phi=0.35, \phi=0.85)$, spectrum, and reconstruction. $\mathrm{H} \gamma$

In Fig. 6.31, the radial intensity dependency is shown. The steeper increase towards the WD for the intensity of $\mathrm{H} \gamma$ is reflected by the broader Doppler tomogram of $\mathrm{H} \gamma$ when compared to $\mathrm{H} \beta$. It can also be seen in the wider line profile of $\mathrm{H} \gamma$.

In the residuals, an identical feature for both emission lines is visible: The emission at $(v, \phi) \approx\left(+600 \mathrm{~km} \mathrm{~s}^{-1}, 0.6 \ldots 0.9\right)$ appears to be too small to be explained with the reconstructed disk. Around $\phi \approx 0.5$ this lack of emission switches from the blue to 

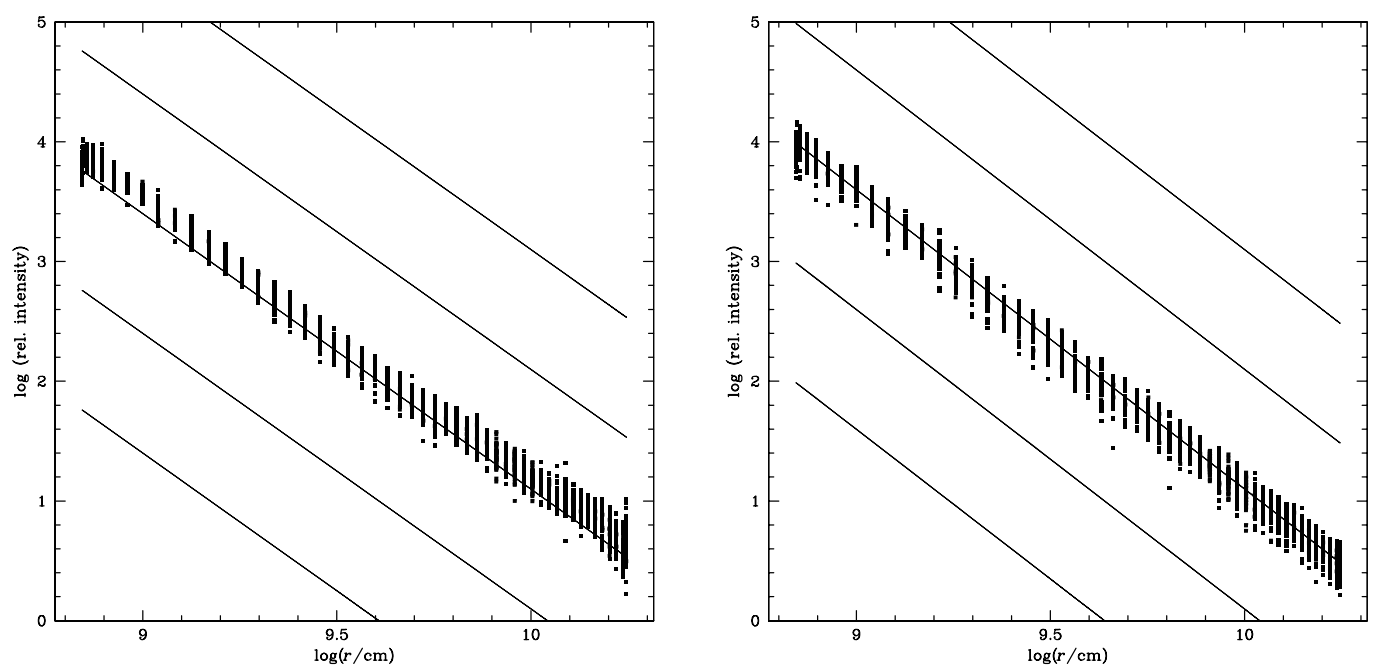

Figure 6.31: V436 Cen: Radial dependency of the intensity. $\mathrm{H} \beta$ (left) and $\mathrm{H} \gamma$ (right). The lines indicate $I \propto r^{-2.3}$ and $I \propto r^{-2.5}$, respectively.

the red side of the line. By only looking at the residuals of the $\mathrm{H} \gamma$ reconstruction, one could also describe the position of the feature as constant at $v \approx+600 \mathrm{~km} \mathrm{~s}^{-1}$ for $\phi=0.6 \ldots 1.3, v$ switiching over to $v \approx-600 \mathrm{~km} \mathrm{~s}^{-1}$ and back between $\phi=0.3$ and $\phi=0.6$.

Since the position of this feature is no sinusoidal emission structure, the OMA algorithm fails to reconstruct it. Moreover, this feature is an lack of emission compared to the disk-only emission. It could be interpreted as an absorption signature like the one observed in HS0455 from an overflowing stream. Attempts to model this absorption feature as the result of an overflowing stream did not lead to satisfying results. Hence, some other effect or a different geometry and physical behaviour of an overflowing accretion stream may be the cause of the V436 Cen assymetric absorption.

Notable is the stability of the results against changes in the system geometry. For all three model geometry sets from Tab. 6.5, the fit results are of the same quality and give the same radial profile of the disk. 


\section{Chapter 7}

\section{Discussion and Summary}

\subsection{The mapping methods}

Since the important works by Horne and Marsh (Horne, 1985; Marsh and Horne, 1988), image reconstruction methods have become popular in the field of CV research. Recently, the number of imaging methods based on either eclipse mapping or Doppler tomography has increased to account for a variety of object classes and data types. In Chapt. 4, I successfully applied the, for accretions disks well established, method of eclipse mapping to CVs without a disk, the highly magnetic polars (Kube et al., 2000). By extending the main idea behind eclipse mapping, the reconstruction of light curves, to a full orbit, I developed the full-orbit eclipse mapping method (Chapt. 5; the method is also called "accretion stream mapping" by the co-inventors Vrielmann and Schwope (2001)). Going further to the reconstruction of time-resolved emission line spectra, I developed the "orbital mapping" method (Kube, 2002; Chapt. 6), which I confronted with the data of two disk systems.

It is feasible to characterize the indirect imaging methods for interacting binary stars in terms of the neccessary input data, the assumptions made by using the specific method, and the resulting maps. This is done in Tab. 7.1.

\subsection{Eclipse mapping}

The eclipse mapping method in its extension to magnetic CVs with their special geometry can be used successfully to trace features along the accretion stream (Chapt. $4)$.

As in all methods described in this thesis, the image is reconstructed by iteratively changing the map of the object to fit the forward synthesized data to the observations. In case of the eclipse mapping method, the data are light curves of emission lines. 
Table 7.1: Comparison of the different indirect imaging methods. Only the most relevant references are given.

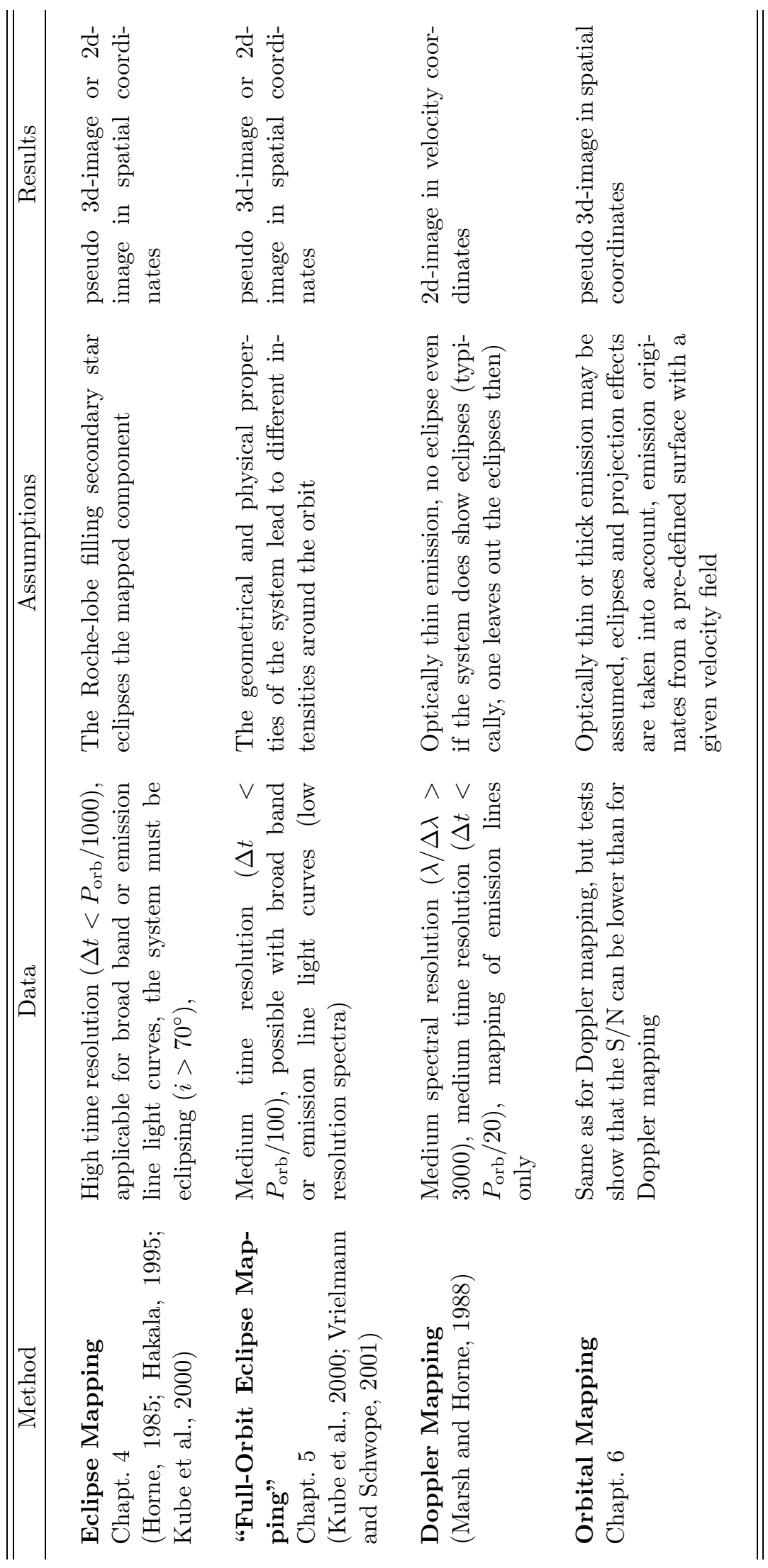


Depending on the geometry of the system, the quality of the fits may be affected by alias effects, which occur if different parts of the accretion stream are eclipsed simultanously (Kube, 2001). This can happen if the WD accretes on two poles or if only ingress or egress data is available. An ideal data set for eclipse mapping in polars would contain light curves of several emission lines or broad band data with a phase resolution of $\Delta \phi \approx 1 / 1000$. That would give 300 . . 400 data points for the typical interval around an eclipse. The algorithm which I use for the image reconstructions is very stable against noise, so a $\mathrm{S} / \mathrm{N}$ of 5 or better is sufficient to allow a reliable reconstruction of the accretion stream map. Due to the limited phase coverage (data available around the eclipse, only), I restrict the intensity variations to the flow direction.

In the analysis of the C IV $\lambda 1550$ line light curve of UZ For in Sect. 4.5, I was able to show that the stream emits both on its ballistic and on its magnetically funneled part. This finding is compatible with Doppler tomograms of UZ For in the light of He II $\lambda 4686$ (Schwope et al., 1999), where emission is detected on locations in velocity space which resemble the position of the two different stream paths. My reconstructions show significantly that there is no emission of $\mathrm{C}$ IV $\lambda 1550$ around the coupling region. The interpretation can be that due to a kinematic stratification of the stream, the matter is heated and ionized up to C IV early in the ballistic stream. Subsequent cooling due to the observed radiations leads to a dark stream again. Smooth coupling to the magnetic field instead of a sudden impact-like breaking fails to heat the matter strongly. The emission on the dipole field line is a result of (a) reprocessed radiation from the accretion spots, which is seen closest to the WD, and (b) material pile-up above the orbital plane, where incomming material fails to ascend against the gravitational potential, stagnates and collides with the following stream material, producing the observed C IV emission by collisional heating.

\subsection{Full-orbit eclipse mapping}

The extension of the eclipse mapping algorithm to the full orbit makes use of geometrical projection effects. Hence, the model has to include the properties of the emission line structure of the surface elements. Observations of the orbital properties of emission lines of polars lead to the assumption that the accretion stream emits optically thick line radiation. The emission of a single surface element $i$ of the accretion stream is described by its emission intensity $I_{i}$ multiplied by the projected surface area $A_{i}$ of that element.

By using the full-orbit light curve, the brightness of the accretion structure can be derived as a $2 \mathrm{~d}$ map: Not only brightness variations along the direction of the accretion flow as in the eclipse maps above, but also variations "around" the stream can be mapped. That allows to find (a) regions of the accretion stream which have a higher surface temperature, which would make them bright at the irradiated surface elements, 
or alternatively (b) extensions of the stream perpendicularly to the flow direction. It can not be distinguished between the both possible results (a) and (b): a bright region seen with the maximum projected area at a given phase $\phi$ is equivalent to a larger surface element at that region, since the contribution of a single surface element $i$ is given by the projected surface area multiplied by the intensity. Physical considerations have to be included in the analysis, as has been done with the interpretation of the results of the FOEM method with the data of HU Aqr in Chapt. 5.

In the case of HU Aqr I was able to reproduce in principle the results of Vrielmann and Schwope (2001), but arrive at a slightly different interpretation of the resulting maps. The map of HU Aqr in the light of the He II $\lambda 4686$ emission line indicates that the accretion stream forms a curtain when it couples to the magnetic field of the WD. That curtain extends in a direction which is in between the original flow direction of the ballistic stream an the direction which is imposed by the magnetic field lines. This may be explained by a combined effect of stripping of stream matter (curtain) and braking of the stream (direction change).

The ideal data set for the FOEM method would contain a full-orbit light curve (ideally emission lines, which can be considered to be optically thick) with at least 50 phase steps. The method is again very robust against noise. Good reconstructions of test maps were reached with $\mathrm{S} / \mathrm{N} \approx 25$, and even for $\mathrm{S} / \mathrm{N} \approx 4$ the fit results were usable.

\subsection{Orbital mapping}

With the newly developed orbital mapping (OMA) method, I presented a forward spectral synthesis code, which allows to create maps of the line emitting regions in a $\mathrm{CV}$ if the geometry is known to a certain precision.

The mapping is again done by iteratively changing the intensity contributions of the surface elements which form the $3 \mathrm{~d}$ grid model of the binary star. From that 3d model, the theoretical spectrum is calculated, taking into account the Doppler shift of the surface elements due to their velocities inside the rotating frame as well as the orbital velocity variations. Arbitrary emission line characteristics can, in principle, be included in the code.

The tests of the code show very promising results if this method is applied to data of accretion disk systems of e.g. dwarf novae, which are assumed to show a symmetric disk with a hot spot. However, such data was not available for my research. I incorporated some extensions to the geometrical model to account for the more complicated geometry of one of the analyzed systems. The creation of the geometrical description is based on physical models of the systems. The quality of the best fit results allows to verify the validity of the physical model. 
I presented applications of the OMA method to two CV systems. The first, HS0455+8315, is a newly discovered novalike system, which shows all the signatures of an SW Sex star. Hence, I tried to device a model which accounts for the current interpretation of the SW Sex phenomenon, an accretion disk modulated by stream overflow. The resulting spectra of this model show fair agreement with the observed data, but can not reproduce all features of the spectral line variation over the orbit. However, I was able to support the assumption that the overflowing stream becomes brighter during the approach to the white dwarf due to heating when it re-impacts on the accretion disk.

The second system which I analyzed with the OMA method, V436 Cen, is an SU UMa star. The spectrum is that of a double-peaked accretion disk with only limited additional features. These features appear to be of absorption character. The absorption resembles that of an overflowing stream. Attempts to incorporate an overflowing stream into the geometry for the OMA method did not reproduce the absorption features. Yet, the OMA method allows to emphasize these features by removing the orbital component of the spectrum. Furter investigation on the source of that absorption is neccessary. The result of the OMA work on V436 Cen is that this systems has a steep intensity profile of the disk, $I \propto r^{-2.3 \ldots-2.5}$ in the Balmer lines.

\subsection{Outlook}

The three methods which I have developed are very stable against noisy data, if the geometry of the mapped systems is known to a fair precision. Hence, they can be used to re-investigate existing data (be it light curves or spectra) which have so far been of too little quality especially for Doppler mapping. For many CVs, full-orbit light curves of different emission lines and broad bands are available. An analysis of these data with the FOEM method will lead to a number of new CV maps.

Since the methods are sensitive to differences between the assumed and the real geometrical configurations, fine-tuning of the orbital parameters and e.g. the velocity profile of accretion disks will lead to a better understanding of the location of emission sources inside CV systems. As my application to HS0455+8315 - a member of the not-yet completely understood SW Sex class of CVs - has shown, the modeling of spectra can help to find discrepancies between the physical models of certain systems and the observed data. Much of this work will be done by synthesizing spectra using the CVMOD code without actually mapping the intensities.

If - on the other hand - the geometry is well known (as for V436 Cen), the OMA method helps to derive some of the intensity data (namely, the intensity map which co-rotates with the system) and to identify parts of the spectrum which contain unexpected or unexplained features. The creation of reliable intensity profiles of accretion disks will be a task for future application of the OMA method. 
In the near future, there will be a number of specialized mapping codes, which focus on the different aspects of interacting binary stars. Clearly, the Doppler mapping method will remain the most general tool for the analysis of spectrally resolved emission lines. Modification of this method are on the horizon: The modulation mapping extension, first presented by Danny Steeghs (2001), allows to drop the assumption that the flux from each point in the binary frame is constant over the orbit. This is done by characterizing the single point in the velocity space not only by it average intensity, but also by amplitude and phase of a possible orbital variation.

A second variation on the theme of Doppler mapping is currently under investigation by Rick Hessman and Bettina Hoffman (Hoffmann et al., 2002). The so-called Kepler tomography leaves the orbital frame and creates images in frames which co-rotate with different accretion annulae of a Keplerian disk. By analyzing many highly time-resolved spectra it will be possible to identify single accretion blobs in the disk.

For eclipsing magnetic systems, Pasi Hakala (Hakala et al., 2002) suggested a way to drop most of the geometric assumptions. By not only fitting the intensity of surface elements of the accretion stream, but also the position of the trajectory, a modelindependent 3d image of polars can be created. However, since the number of degrees of freedom is very high in this mapping method, the results have to be looked at with great care.

The next logical step in the mapping codes will be to leave the locations and the velocities of emission sources unrestricted. With high quality data from spectroscopy at the new $8 \mathrm{~m}$-class telescopes and evolution strategies on fast parallel computers, assumption-free $3 \mathrm{~d}$ maps of cataclysmic variables may be generated in the next decade.

Until then, further projects for FOEM and OMA will contain applications of FOEM with accretion disk systems and vice versa of OMA with data of magnetic systems. The high spatial resolution of OMA and FOEM and its sensitivity to model discrepancies will make these methods to touchstones of the understanding of emission sources in CVs. 


\section{Appendix A}

\section{CVcat - An Interactive Database of Cataclysmic Variables and Related Objects}

CVcat is an interactive database or "online catalogue", which offers features so far unknown to scientific catalogues. It was developed as a tool for the CV community and as a case study for some of the concepts. Its original name - TPP - reminds of the very early stages when the catalogue was planned to be used for polars: TPP stands for "The Polar Page". CVcat can be found online at http://cvcat.org. It was presented to the public in August 2001 at the CV conference in Göttingen (Kube et al., 2002). Since then, the number of users of CVcat has increased to 89, the daily average of requests in March 2002 was 30 (delivered result pages).

\section{A.1 The concept}

CV cat differs from other catalogues or data bases by the principle of the data input. So far, all available catalogues (e.g. Downes et al., Ritter and Kolb) have a limited editorial team, which publishes updated versions of the catalogue from time to time. The catalogue offers exactly one value for each listed property (e.g. distance, orbital period) and object.

In CVcat, every registered user may add data to the catalogue, which is instantly visible for all other users. The quality control is done by an editorial team, which may alter or remove erroneous data. For every property of an object, an arbitrary number of values can be stored (e.g. several published distances). One of these values should be marked as the best available value by one of the editors. 
Every value stored in CVcat is connected to the publication where it originates from by a link to the appropriate ADS site, using the ADS bibcode of the paper. Hyperlinks to VSNET messages and astro-ph preprints are also included. Additionally, publications which do not contain single derived values can be stored in CVcat.

Data retrieval from CVcat can work in mainly two ways: (i) the user gets all available data for a specific object (which can be found using its object type, its coordinates, or one of its names), (ii) the user creates a table of some properties of a list of objects. The latter method allows to create data tables suitable for easy graphic generation as well as ready-to-publish LaTeX-tables.

In CVcat, data for all objects of the classes described in Sect. 2.2 can be stored. For every object, the following properties can be included: hydrogen column density, general magnitude, high state magnitude, low state magnitude, optical light curve exists, magnitude in eclipse, eclipsing, distance, inclination, primary mass, secondary mass, mass ratio, primary radius, secondary radius, secondary spectral type, orbital ephemeris, spin ephemeris, orbital period, spin period, colatitude of a primary magnetic pole, azmimuth of a primary magnetic pole, field strength of a primary magnetic pole, colatitude of a secondary magnetic pole, azmimuth of asecondary magnetic pole, field strength of a secondary magnetic pole, optical spectrum exists (reference only), superhump period, primary temperature, general magnetic field strength of primary star, uv data (reference only), x-ray data (reference only), doppler tomogram (reference only), eclipse map (reference only), secondary temperature.

\section{A.2 Technical realization}

CVcat is built as a Perl script, which runs on a Linux PC. This Perl script processes the user requests (HTTP GET/POST requests) and creates HTML pages, which are delivered via the Apache web server. Hence, CVcat presents itself as an interactive web page.

The data is stored in a MySQL data base to which the Perl script communicates using the standard Perl DBI/DBD interface.

\section{A.3 Prospects}

The concept of CVcat has demonstrated the value of a centrally administrated catalogue with a globally shared expertise of the users. The general structure of CVcat is also applicable to other fields of astronomy. Hence, a more general software called "AstroCat" based on the experiences with CVcat will be developed. This software will also 
include more features to improve the knowledge management of astronomical results and data:

- besides the "single number data" stored so far, the infrastructure for storing light curves, spectra, and finding charts will be added,

- information agents will allow users to be informed automatically about new data in the database for the objects of interest for the user,

- a sophisticated validation system will allow the users to add their comments to published data,

- data which has not yet been cross-checked by the editorial team will be tagged as such,

- hyperlinks to any possible web ressource will be included for every object 


\title{
Appendix B
}

\section{Symbols and Acronyms}

\author{
2MASS Two Micron All Sky Survey \\ a distance between star centers, "orbital separation" \\ $a_{1}, a_{2} \quad$ distance from the center of the primary/secondary star to the center of gravity \\ $A_{i} \quad$ surface area of a surface element \\ $A_{i}(\phi) \quad$ projected surface area of a surface element \\ $\Delta A_{i} \quad$ accumulated change of the projected surface area of the $i$-th surface element \\ ADS Astrophysics Data System \\ $B \quad$ magnetic field strength \\ c speed of light, $c=299792458 \mathrm{~km} \mathrm{~s}^{-1}$ \\ CV cataclysmic variable binary star \\ CVcat catalogue of CVs \\ CVMOD CV model code of this work \\ $d, d^{\prime} \quad$ number of dimensions \\ $D_{i} \quad$ default image intensity of the $i$-th surface element \\ DBI/DBD database interface/database driver \\ EM eclipse mapping \\ EUVE Extreme Ultraviolet Explorer \\ FOEM full-orbit eclipse mapping \\ FWHM full width at half maximum, in case of a Gaussian: \\ $\mathrm{FWHM}=\sqrt{8 \ln 2} \cdot \sigma \approx 2.355 \cdot \sigma$ \\ $F \quad$ observed flux \\ $F_{\lambda} \quad$ observed flux at wavelength $\lambda$ \\ FORS2 FOcal Reducer/low dispersion Spectrograph at the VLT \\ FOS Faint Object Spectrograph on the HST \\ $G \quad$ gravitational constant, $G=6.67259(85) \cdot 10^{-8} \mathrm{~cm}^{3} \mathrm{~g}^{-1} \mathrm{~s}^{-2}$ \\ GHRS Goddard High Resolution Spectrograph on the HST \\ HIT High Time Resolution modes for FORS2
}




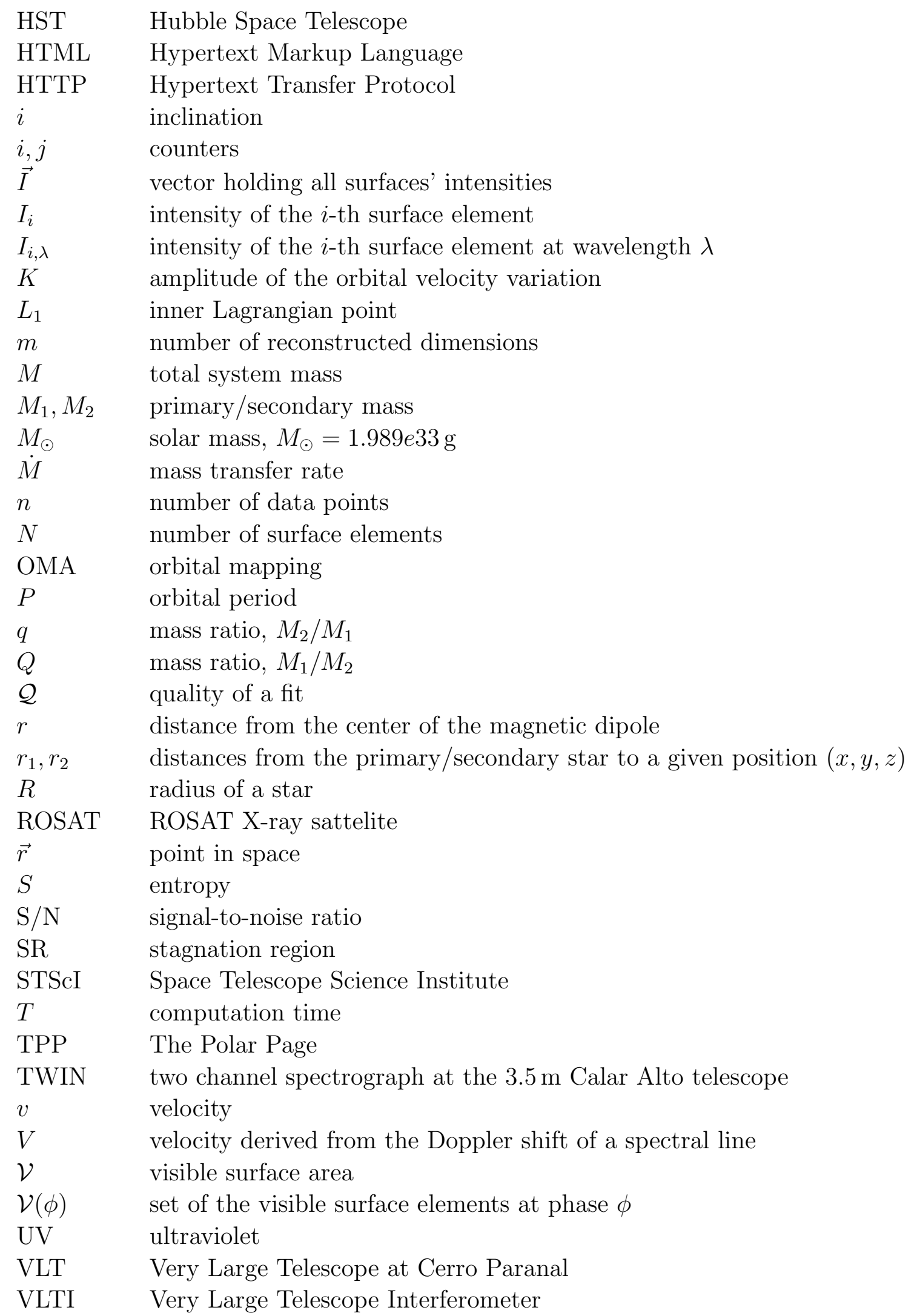




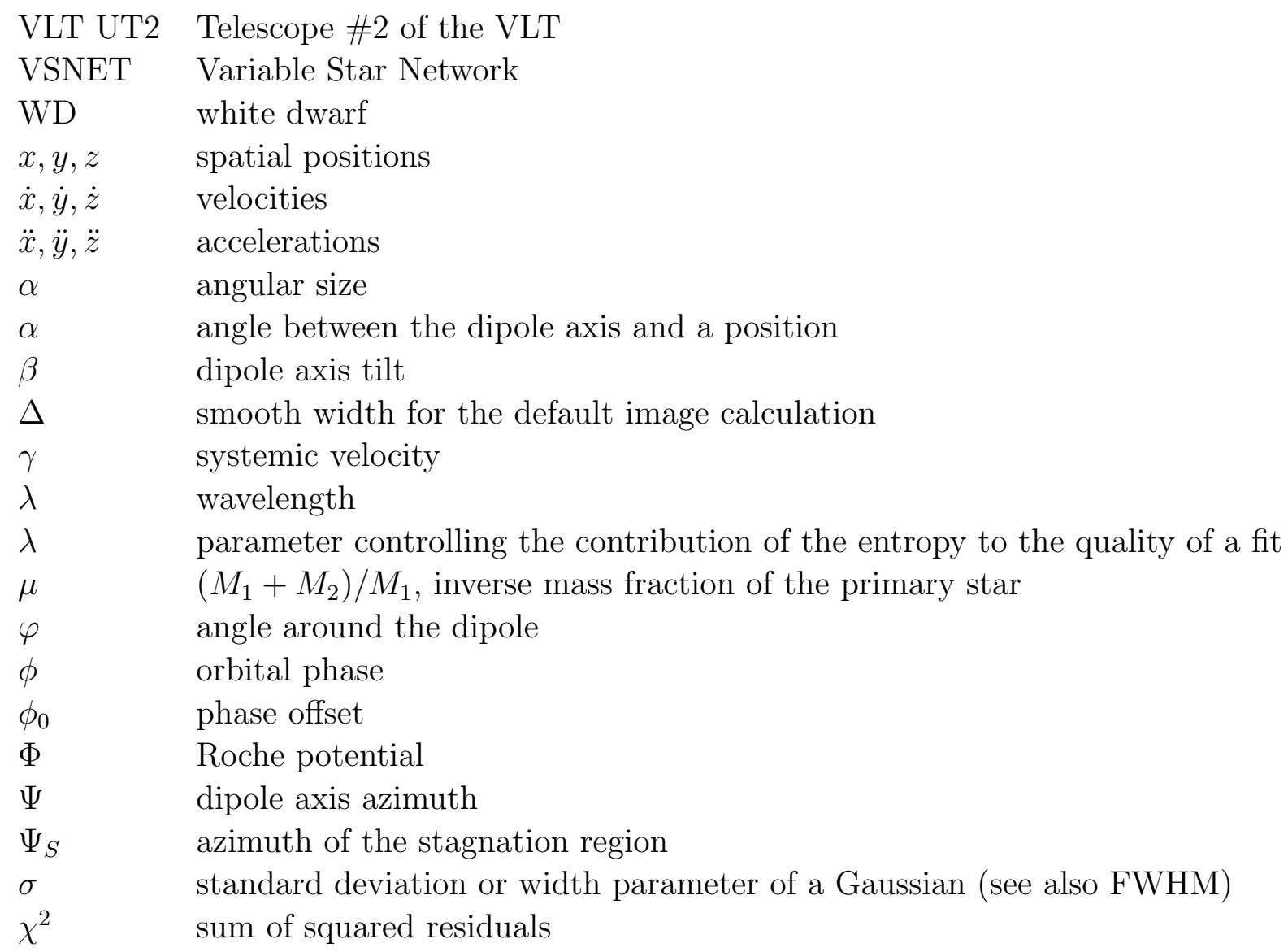


APPENDIX B. SYMBOLS AND ACRONYMS 


\section{Bibliography}

Armitage, P. J. and Livio, M., 1996, ApJ 470, 1024

Augusteijn, T., van Kerkwijk, M. H., and van Paradijs, J., 1993, A\&A 267, L55

Bailey, J., 1979, MNRAS 188, 681

Bailey, J., 1995, in B. Warner and D. A. H. Buckley (eds.), Fundamental Properties of Polars, No. 85 in ASP Conference Series, pp 10-20, Astronomical Society of the Pacific, San Francisco

Bailey, J. and Cropper, M., 1991, MNRAS 253, 27

Baraffe, I., Chabrier, G., Allard, F., and Hauschildt, P. H., 1998, A\&A 337, 403

Berriman, G. and Smith, P. S., 1988, ApJ 329, L97

Beuermann, K., Thomas, H.-C., and Schwope, A., 1988, A\&A 195, L15

Boffin, H., Steeghs, D., and Cuypers, J. (eds.), 2001, Astrotomography - Indirect Imaging Methods in Observational Astronomy, No. 573 in Lecture notes in physics, Berlin, Heidelberg, Springer-Verlag Berlin Heidelberg New York

Bond, I. A., Bond, I. A., and Freeth, R. V., 1988, MNRAS 232, 753

Charbonneau, P., 1995, ApJS 101, 309

Ciardi, D. R., Howell, S. B., Hauschildt, P. H., and Allard, F., 1998, ApJ 504, 450

Cropper, M. and Warner, B., 1986, MNRAS 220, 633

de Jager, O. C., Meintjes, P. J., O’Donoghue, D., and Robinson, E. L., 1994, MNRAS 267, 577

Deans, S. R., 1993, The Radon Transform and Some of Its Applications, Krieger Publishing Company, Malabar, Florida

Dorward, S. E., 1994, International Journal of Computational Geometry \& Applications 4, 325

Echevarria, J., 1983, Revista Mexicana de Astronomia y Astrofisica 8, 109 
Flannery, B. P., 1975, ApJ 201, 661

Frank, J., King, A. R., and Raine, D. J., 1992, Accretion power in astrophysics, Cambridge astrophysics series, Cambridge University Press, Cambridge, 2nd edition

Gänsicke, B. T., Beuermann, K., and Reinsch, K. (eds.), 2002a, The Physics of Cataclysmic Variables and Related Objects, Göttingen, August 5-10 2001, No. 261 in ASP conference series, ASP, in press

Gänsicke, B. T., Hagen, H.-J., Kube, J., Schwarz, R., A., S., Engels, D., Nogami, D., and Kuduz, M., 2002b, in (Gänsicke et al., 2002a), pp 623-624

Gänsicke, B. T., Hoard, D. W., Beuermann, K., Sion, E. M., and Szkody, P., 1998, A\&A 338, 933

Garnavich, P. M., Szkody, P., Mateo, M., Feinswog, L., Booth, J., Goodrich, B., Miller, H. R., Carini, M. T., and Wilson, J. W., 1990, ApJ 365, 696

Gilliland, R. L., 1982, ApJ 254, 653

Glindemann, A., Abuter, R., Carbognani, F., Delplancke, F., Derie, F., Gennai, A., Gitton, P. B., Kervella, P., Koehler, B., Leveque, S. A., Menardi, S., Michel, A., Paresce, F., Duc, T. P., Richichi, A., Schoeller, M., Tarenghi, M., Wallander, A., and Wilhelm, R., 2000, in Proc. SPIE, Interferometry in Optical Astronomy, Pierre J. Lena; Andreas Quirrenbach; Eds., Vol. 4006, pp 2-12

Hagen, H.-J., Groote, D., Engels, D., and Reimers, D., 1995, A\&AS 111, 195

Hakala, P., Cropper, M., and Ramsay, G., 2002, in (Gänsicke et al., 2002a), pp $153-154$

Hakala, P. J., 1995, A\&A 296, 164

Hameury, J. M., King, A. R., and Lasota, J. P., 1986, MNRAS 218, 695

Hameury, J. M., King, A. R., and Lasota, J. P., 1988, A\&A 195, L12

Harrop-Allin, M. K., Cropper, M., Hakala, P. J., Hellier, C., and Ramseyer, T., 1999a, MNRAS 308, 807

Harrop-Allin, M. K., Hakala, P. J., and Cropper, M., 1999b, MNRAS 302, 362

Heerlein, C., Horne, K., and Schwope, A. D., 1999, MNRAS 304, 145

Hellier, C., 1996, ApJ 471, 949

Hellier, C., 2000, New Astronomy Reviews 44, 131

Hellier, C., 2001, Cataclysmic Variable Stars - How and why they vary, Springer-Praxis books in astronomy and space sciences, Springer Verlag Berlin Heidelberg New York, Praxis Publishing Ltd, Chichester

Hellier, C. and Mukai, K. (eds.), 1999, Annapolis Workshop on Magnetic Cataclysmic Variables, No. 157 in ASP Conference Series, San Francisco, Astronomical Society of the Pacific 
Hellier, C. and Robinson, E. L., 1994, ApJ 431, 107

Hessman, F. V., Gänsicke, B. T., and Mattei, J. A., 2000, A\&A 361, 952

Hoard, D. W., 1998, Ph.D. thesis, University of Washington

Hoard, D. W. and Szkody, P., 1997, ApJ 481, 433

Hoffmann, B., Hessman, F. V., and Reinsch, K., 2002, in (Gänsicke et al., 2002a), pp 485-486

Horne, K., 1985, MNRAS 213, 129

Horne, K., 1999, in (Hellier and Mukai, 1999), pp 349

Horne, K., 2001, in (Boffin et al., 2001), pp 386-415

Horne, K., Welsh, W. F., and Peterson, B. M., 1991, ApJ 367, L5

Huber, M. E., Howell, S. B., Ciardi, D. R., and Fried, R., 1998, PASP 110, 784

King, A. R., 1993, MNRAS 261, 144

King, A. R. and Schenker, K., 2002, in (Gänsicke et al., 2002a), pp 233-241

Kippenhahn, R. and Weigert, A., 1990, Stellar Structure and Evolution, A\&A

Library, Springer-Verlag, Berlin, Corrected 3rd printing 1994

Knigge, C., Long, K. S., Hoard, D. W., Szkody, P., and Dhillon, V. S., 2000, ApJ 539, L49

Kolb, U., 2002, in (Gänsicke et al., 2002a), pp 180-189

Kube, J., 2001, in (Boffin et al., 2001), pp 175-182

Kube, J., 2002, in (Gänsicke et al., 2002a), p. 495

Kube, J., Gänsicke, B. T., and Beuermann, K., 2000, A\&A 356, 490

Kube, J., Gänsicke, B. T., and Beuermann, K., 1999, in (Hellier and Mukai, 1999), pp 99-103

Kube, J., Gänsicke, B. T., and Hoffmann, B., 2002, in (Gänsicke et al., 2002a), pp $678-679$

Kunze, S., Speith, R., and Hessman, F. V., 2001, MNRAS 322, 499

Lázaro, F. C. and Arévalo, M. J. (eds.), 2001, Binary Stars: Selected Topics on Observations and Physical Processes, No. 563 in Lecture notes in physics, Berlin, Heidelberg, Springer-Verlag Berlin Heidelberg New York

Lynden-Bell, D. and Pringle, J. E., 1974, MNRAS 168, 603

Marsh, T. R., 2001, in (Lázaro and Arévalo, 2001), pp 151-202

Marsh, T. R. and Horne, K., 1988, MNRAS 235, 269

McDermott, P. N. and Taam, R. E., 1989, ApJ 342, 1019 
Nadalin, I. and Sion, E. M., 2001, PASP 113, 829

Nauenberg, M., 1972, ApJ 175, 417

O'Brien, K. and Horne, K., 2001, in (Boffin et al., 2001), pp 416-423

Osborne, J. P., Giommi, P., Angelini, L., Tagliaferri, G., and Stella, L., 1988, ApJ $328, \mathrm{~L} 45$

Paczynski, B., 1977, ApJ 216, 822

Patterson, J., 1984, ApJS 54, 443

Rappaport, S., Joss, P. C., and Verbunt, F., 1983, ApJ 275, 713

Rechenberg, I., 1994, Evolutionsstrategie '94, No. 1 in Werkstatt Bionik und Evolutionstechnik, frommann-holzboog, Stuttgart

Rousseau, T., Fischer, A., Beuermann, K., and Woelk, U., 1996, A\&A 310, 526

Schenker, K. and King, A. R., 2002, in (Gänsicke et al., 2002a), pp 242-251

Schreiber, M. R., Gänsicke, B. T., and Mattei, J. A., 2002, A\&A 384, L6

Schwope, A., Beuermann, K., and Thomas, H.-C., 1990, A\&A 230, 120

Schwope, A., Mantel, K.-H., and Horne, K., 1997a, A\&A 319, 894

Schwope, A., Mengel, S., and Beuermann, K., 1997b, A\&A 320, 181

Schwope, A. D., Schwarz, R., Staude, A., Heerlein, C., Horne, K., and Steeghs, D., 1999, in (Hellier and Mukai, 1999), pp 71-80

Sekiguchi, K., 1992, Nature 358, 563

Shafter, A. W., 1983, Ph.D. Thesis

Shafter, A. W. and Szkody, P., 1984, ApJ 276, 305

Shakura, N. I. and Sunyaev, R. A., 1973, A\&A 24, 337

Sirk, M. M. and Howell, S. B., 1998, ApJ 506, 824

Skilling, J. and Bryan, R. K., 1984, MNRAS 211, 111

Smak, J., 2001, in (Lázaro and Arévalo, 2001), pp 110-150

Smith, D. A. and Dhillon, V. S., 1998, MNRAS 301, 767

Smith, D. A., Dhillon, V. S., and Marsh, T. R., 1998, MNRAS 296, 465

Spruit, H. C., 1994, A\&A 289, 441

Spruit, H. C. and Ritter, H., 1983, A\&A 124, 267

Steeghs, D., 2001, in (Boffin et al., 2001), pp 45-68

Stockman, H. S. and Schmidt, G. D., 1996, ApJ 468, 883 
Szkody, P. and Piche, F., 1990, AJ 361, 235

Thorstensen, J. R., Davis, M. K., and Ringwald, F. A., 1991, AJ 102, 683

Vogt, S. S. and Penrod, G. D., 1983, PASP 95, 565

Vrielmann, S., Horne, K., and Hessman, F. V., 1999, MNRAS 306, 766

Vrielmann, S. and Schwope, A. D., 1999, in (Hellier and Mukai, 1999)

Vrielmann, S. and Schwope, A. D., 2001, MNRAS 322, 269

Warner, B., 1995a, Cataclysmic Variable Stars, No. 28 in Cambridge Astrophysics Series, Cambridge University Press, Cambridge

Warner, B., 1995b, Dwarf Novae, Chapt. 2, in (Warner, 1995a)

Warren, J. K., Sirk, M. M., and Vallerga, J. V., 1995, ApJ 445, 909

Wood, J., Horne, K., Berriman, G., Wade, R., O’Donoghue, D., and Warner, B., 1986, MNRAS 219, 629

Wynn, G., 2001, in (Boffin et al., 2001), pp 155-174

Wynn, G. A. and King, A. R., 1995, MNRAS 275, 9 


\section{List of Tables}

4.1 System geometry of the imaginary system IM Sys . . . . . . . . . . . . 32

4.2 Quality and number of iterations for the test calculations. . . . . . . . 34

4.3 System geometry of UZ Fornacis . . . . . . . . . . . . . . . . . 38

5.1 System parameters for the FOEM test runs. . . . . . . . . . . . . . . . 48

6.1 System parameters of the synthetic object CV Toy . . . . . . . . . 62

6.2 Eclipse widths . . . . . . . . . . . . . . . . 75

6.3 Eclipse data of $\mathrm{HS} 0455 \ldots \ldots \ldots$. . . . . . . . . . . . . 76

6.4 Emission lines found in the HS0455-spectrum . . . . . . . . . . . 80

6.5 Model geometry for V436 Cen . . . . . . . . . . . . . . . . 102

7.1 Comparison of the imaging methods . . . . . . . . . . . . . . 110 


\section{List of Figures}

2.1 Roche equipotential surfaces . . . . . . . . . . . . . . 5

2.2 Relative numbers of CVs . . . . . . . . . . . . . . . . . 10

2.3 Secondary star masses and period histogram . . . . . . . . . . . . . 12

3.1 The principle of tomography . . . . . . . . . . . . . . . . 14

3.2 The smallest possible surface elements . . . . . . . . . . . . . . 17

3.3 Doppler coordinates . . . . . . . . . . . . . . . . . . . . . . 20

4.1 Artist's view of a polar . . . . . . . . . . . . . . . 26

$4.23 \mathrm{~d}$ grid model . . . . . . . . . . . . . . . . . . . . 27

4.3 Synthetic stream reconstructions (point) . . . . . . . . . . . . . 33

4.4 Synthetic stream plots (point) . . . . . . . . . . . . . . . 34

4.5 Synthetic stream reconstructions (zebra) . . . . . . . . . . . 35

4.6 Synthetic stream plots (zebra) . . . . . . . . . . . . . . . 36

4.7 Trailed spectrum of UZ For . . . . . . . . . . . . . . . . . . . . . 39

4.8 Extracted C IV light curves and best fits . . . . . . . . . . . . . 40

4.9 Resulting UZ For maps I . . . . . . . . . . . . . . . . . . . . . 40

4.10 Resulting UZ For maps II . . . . . . . . . . . . . . . . . . . . . . . 40

4.11 Results for orbit $1 \ldots \ldots$. . . . . . . . . . . . . 41

4.12 Dipole field line configuration . . . . . . . . . . . . . . . . 43

5.1 Information content for HU Aqr . . . . . . . . . . . . . . . . . 47

5.2 Synthetic light curve and FOEM reconstruction I . . . . . . . . . . . . 50

5.3 Synthetic light curve and FOEM reconstruction II . . . . . . . . . . . . 50

5.4 Synthetic light curve and FOEM reconstruction III . . . . . . . . . . 51 
5.5 Synthetic light curve and FOEM reconstruction IV . . . . . . . . . . 52

5.6 Synthetic light curve and FOEM reconstruction V . . . . . . . . . . 53

5.7 Synthetic light curve and FOEM reconstruction VI . . . . . . . . . . 53

5.8 HU Aqr line emission and reconstruction . . . . . . . . . . . . . . 54

5.9 3d sequence of $\mathrm{HU}$ Aqr . . . . . . . . . . . . . . . . . . 56

5.10 Model for the HU Aqr curtain . . . . . . . . . . . . . . . . . . . 57

6.1 Synthetic OMA I . . . . . . . . . . . . . . . . 65

6.2 Synthetic OMA II . . . . . . . . . . . . . . . . . . . . . . . 66

6.3 Synthetic OMA III . . . . . . . . . . . . . . . . . . . . . . . . . . . 68

6.4 Synthetic OMA IV . . . . . . . . . . . . . . . . . . . . . . . . . 69

6.5 Synthetic OMA IV . . . . . . . . . . . . . . . . . . . 70

6.6 Traces trough the toy model disk . . . . . . . . . . . . . . . . . 71

6.7 Synthetic OMA V . . . . . . . . . . . . . . . . 72

6.8 Synthetic OMA VI . . . . . . . . . . . . . . . . 73

6.9 R light curves of HSO455+83 . . . . . . . . . . . . . . . 77

6.10 Calar Alto TWIN-spectrum of HS0455+83 . . . . . . . . . . . . . 78

6.11 Blue and red part of the Calar Alto TWIN-spectrum of HS0455+83 . . 79

6.12 The hydrogen emission lines in HS0455 . . . . . . . . . . . . . . 81

6.13 The spectra in $\mathrm{H} \alpha$ during the eclipse . . . . . . . . . . . . . . . . 83

6.14 The spectrum of He II . . . . . . . . . . . . . . . . . . . . . 84

6.15 Center of the He II $\lambda 4686$ emission line . . . . . . . . . . . . . . . 85

6.16 SW Sex line components . . . . . . . . . . . . . . . 85

6.17 The spectra in $\mathrm{H} \alpha$ over the complete orbit . . . . . . . . . . . . 86

6.18 The spectra in He II $\lambda 4686$ over the complete orbit . . . . . . . . . . 87

$6.19 \mathrm{H} \alpha$ and He II $\lambda 4686$ light curve . . . . . . . . . . . . . . . 88

6.20 Doppler tomogram of $\mathrm{HS} 0455, \mathrm{H} \alpha \ldots \ldots \ldots$

6.21 Doppler tomogram of HS0455, He II . . . . . . . . . . . . . . . . . . 90

6.22 Model geometry for HS0455 . . . . . . . . . . . . . . . . . . . . . 92

6.23 HS0455 fit result . . . . . . . . . . . . . . . . . . . . 96

$6.24 \mathrm{HS} 0455$ fit result $\ldots \ldots \ldots \ldots$. . . . . . . . . . . . . . 97

6.25 HS0455 fit result . . . . . . . . . . . . . . . . . . . . 98 
$6.26 \mathrm{HS} 0455$ fit traces $\ldots \ldots \ldots$. . . . . . . . . . . . . . . 99

6.27 V436 Cen integrated lines . . . . . . . . . . . . . . . 104

6.28 V436 Cen Doppler tomograms . . . . . . . . . . . . . . . 104

6.29 V436 Cen, $\mathrm{H} \beta \ldots \ldots \ldots \ldots$. . . . . . . . . . . . . 105

$6.30 \mathrm{~V} 436 \mathrm{Cen}, \mathrm{H} \gamma \ldots \ldots \ldots \ldots$. . . . . . . . . . . . . 106

6.31 V436 Cen intensity profiles . . . . . . . . . . . . . . . 107 


\section{Publications}

\section{Refereed publications}

Kube, J.: The Geometrical Configuration of Polars and Possible Reconstruction Artefacts of Eclipse Mapping Methods. Astrotomography, Indirect Imaging Methods in Observational Astronomy, Edited by H.M.J. Boffin, D. Steeghs and J. Cuypers, Lecture Notes in Physics, vol 573 (2001), pp 175-181

Kube, J., Gänsicke, B. T., Beuermann, K.: Eclipse mapping of the accretion stream in UZ Fornacis. Astronomy and Astrophysics, vol 356 (2000), pp 490-500

\section{Conference contributions}

Kube, J.: Orbital Mapping: A Combination of Eclipse and Doppler Mapping. The Physics of Cataclysmic Variables and Related Objects, edited by B. T. Gänsicke, K. Beuermann and K. Reinsch, ASP conference series, vol 261 (2002), p 495

Gänsicke, B. T., Hagen, H.-J., Kube, J., Schwarz, R., Staude, A., Engels, D., Nogami, D., Kuduz, M.: HS 0455+8315: A new eclipsing novalike variable. The Physics of Cataclysmic Variables and Related Objects, edited by B. T. Gänsicke, K. Beuermann and K. Reinsch, ASP conference series, vol 261 (2002), p 623

Kuduz, M., Reinsch, K., Beuermann, K. and Kube, J.: Detection of circumbinary material in the galactic supersoft X-Ray binary $Q R$ And. The Physics of Cataclysmic Variables and Related Objects, edited by B. T. Gänsicke, K. Beuermann and K. Reinsch, ASP conference series, vol 261 (2002), p 641

Kube, J., Gänsicke, B. T. and Hoffmann, B.: TPP - An Interactive Database of Cataclysmic Variables and Related Objects. The Physics of Cataclysmic Variables and Related Objects, edited by B. T. Gänsicke, K. Beuermann and K. Reinsch, ASP conference series, vol 261 (2002), p 678

Kube, J.: The Geometrical Configuration of Polars and Possible Reconstruction Artefacts of Eclipse Mapping Methods. Astrotomography, Indirect Imaging Methods in Observational Astronomy, Edited by H.M.J. Boffin, D. Steeghs and J. Cuypers, Lecture Notes in Physics, vol 573 (2001), pp 175-182 
Reinsch, K., Beuermann, K., Kube, J., Kuduz, M., Burwitz, V.: Optical Spectroscopy of two Double-degenerate Polar Candidates. Astronomische Gesellschaft Abstract Series, Abstracts of Contributed Talks and Posters presented at the Annual Scientific Meeting of the Astronomische Gesellschaft at Bremen, September 18-23, 2000, vol 17 (2000), p 67

Kube, J., Gänsicke, B. T., Beuermann, K.: The Accretion Streams in UZ For and V1309 ORI. AG Abstract Services, Abstracts of Contributed Talks and Posters presented at the Annual Scientific Meeting of the Astronomische Gesellschaft, in Goettingen, 20-25 September 1999, vol 15 (1999), p 05

Kube, J., Gänsicke, B. T., Beuermann, K.: Observing the Accretion Stream in Polars. Annapolis Workshop on Magnetic Cataclysmic Variables, edited by Coel Hellier and Koji Mukai, ASP Conference Series, vol 157 (1999), p 99

Kube, J., Gänsicke, B. T., Beuermann, K.: Prediction of Light Curves from Polars. Astronomische Gesellschaft Meeting Abstracts, Abstracts of Contributed Talks and Posters presented at the Annual Scientific Meeting of the Astronomische Gesellschaft at Heidelberg, September 14-19, vol 14 (1998), p 07

Kube, J., Gänsicke, B. T., Beuermann, K.: Mapping the Accretion Stream in Polars. Astronomische Gesellschaft Meeting Abstracts, Abstracts of Contributed Talks and Posters presented at the Annual Scientific Meeting of the Astronomische Gesellschaft at Heidelberg, September 14-19, vol 14 (1998), p 40

\section{Editorial duties}

Together with Boris Gänsicke, I form the editorial team of the interacting binaries newsletter "IBnews". IBnews is being published electronically roughly every 3 months since January 2000. I have been the editor-in-chief of the odd numbered issues except of \#5. IBnews can be found online at http://www. uni-sw.gwdg.de/ibnews/. 


\section{Acknowledgements}

I thank Klaus Beuermann and Boris Gänsicke for the freedom I had with my work.

Chapter 4: Hans-Christoph Thomas discussed with me on the geometry of the accretion stream. Andreas Fischer gave general comments on this work. André Van Teeseling had some ideas how to test the algorithm.

Chapter 5: Axel Schwope kindly provided me with the reduced data of HU Aqr.

Chapter 6: Boris Gänsicke discovered HS0455 in his search for CVs in the Hamburg-SchmidtSurvey. He helped with the calibration of my Calar Alto data. Bettina Hoffman helped me with some MIDAS issues and asked many critical questions. Matthias Schreiber gave me some insights into accretion disks and helped with Super Mongo. Mario Kuduz assisted in the observations of HS0455 on Calar Alto. Robert Schwarz and Andreas Staude made some photometry of HS0455. For HS0455 I used the Doppler mapping code from Henk Spruit. For the section on V436 Cen I used data taken at the VLT by Klaus Reinsch for a project by Rick Hessman and Bettina Hoffmann. Bettina Hoffmann calculated the Doppler maps of V436 Cen.

Appendix A: Much of the input for the design of CVcat came from Boris Gänsicke and Bettina Hoffman. Domitilla de Martino, Boris Gänsicke, Don Hoard, Steve Howell, Tom Marsh, Klaus Reinsch, Matthias Schreiber, and John Thorstensen volunteered to be responsible editors for some object types in CVcat.

I thank Hergen Schultze and Boris Gänsicke for proof reading of this thesis.

Most of the software used for this work was written and controlled using "free" tools like gcc, Emacs, Perl, Tcl/Tk, Linux, MySQL, teTeX, Ghostview, Ghostscript, Xfig to name the most important. Besides these free tools, Compaq/Digital computers with the Tru64 operating system were used with their native C-compiler. Most of the plots were made with Super Mongo. For the evolution strategy, I used the evoC code from the TU Berlin The system administrators of the observatory, especially Klaus Reinsch and Peter Weilbacher, did their best to keep the systems running. Martin Feix und Jens Chluba showed me their LaTeX code for PDF and color generation. Barbara La Scala provided me with the quote from "Mission of Gravity".

This work was funded by the DLR under contract 50 OR 99036.

For the good times at work and beyond and for the encouragement I thank my colleagues, my friends, and my parents. 


\section{Curriculum Vitae}

Name

Date of Birth

Parents

Nationality

Marital status

$8 / 1981-7 / 1985$

$8 / 1985-7 / 1994$

10/1994-7/1996

8/1996-9/1996

10/1996-2/1999

the "Diplom" in physics (very good, 1.1) at the Univerthe "Diplom" in physics (very good, 1.1) at the Universitätssternwarte. Title of the diploma thesis: "Kartierung des Akkretionsstroms in wechselwirkenden Doppelsternen"

2/1998-1/2001 Elected member of the students' administration and the students' parliament in the physics faculty

2/2000-1/2001 Elected member of the students' parliament of the University of Göttingen

since 3/1999 Research assistant at the Universitätssternwarte Göttingen. PhD studies in Astronomy. Supervisor: Prof. Dr. Klaus Beuermann. 
